\title{
APLICACIÓN DE MÉTODOS DE ORBITALES MOLECULARES AL ESTUDIO DE LA OXIDACIÓN TROPOSFÉRICA DE PARAFINAS E HIDROCARBUROS AROMÁTICOS
}

Tesis que presenta Isidoro Garcia Cruz para obtener el grado de Doctor en Ciencias Diciembre, 1999

\author{
UNIVERSIDAD AUTONOMA METROPOLITANA \\ IZTAPALAPA
}

DIVISIÓN DE CIENCIAS BÁSICAS E INGENIERÍAS 


\title{
APLICACIÓN DE MÉTOdOS DE ORBITALES MOLECULARES AL ESTUDIO DE LA OXIDACIÓN TROPOSFÉRICA DE \\ PARAFINAS E HIDROCARBUROS AROMÁTICOS
}

Tesis que presenta

Isidoro Garcia Cruz

para obtener el grado de

Doctor en Ciencias

Diciembre, 1999

Revisado por:

\author{
Dra. Ana Maria Vivier (UAM-I) \\ Director de tesis \\ $\overline{\text { Dr. Rodolfo Esquivel Olea (UAM-I) }}$ \\ Presidente \\ Dr. Marcelo Galván Espinoza (UAM-I) \\ Secretario \\ Dra. Maria Esther Ruiz Santoyo (IMP) \\ Vocal \\ Dr. Salvador Cruz Jiménez (UAM-I/IMP) \\ Vocal \\ $\overline{\text { Dr. Miguel Castro Martínez (UNAM) }}$ \\ Vocal
}


Este trabajo de tesis se desarrolló en el Area de Química Cuántica del Departamento de Química de la UAM Iztapalapa, bajo la dirección de la Dra. Ana Maria Vivier, en coordinación con la Dra. Maria Esther Ruiz del Instituto Mexicano del Petróleo. 
A Maria del Carmen mi esposa, por todo el amor que me ha brindado,

A Juanito mi hijo, que es mi alegría y la razón de mi existencia, deuda,

A mis padres, hermanos y sobrinos, con quienes siempre estoy en

...gracias, muchas gracias. 


\section{AGRADECIMIENTOS}

Agradezco profundamente a la Dra. Annik Vivier Jegoux, todo el tiempo, el esfuerzo y la dirección siempre adecuada de este trabajo. Gracias Annik, porque a fuerza de entenderme ayudaste a superarme.

Un especial agradecimiento a mis amigos, los Profesores, Pancracio Palting, Alfonso Hernández Laguna y Raúl Alvarez-Idaboy, por toda la ayuda que me brindaron durante el desarrollo de mi tesis.

Mi más sincero reconocimiento y agradecimiento al Dr. Rodolfo Esquivel Olea, Dr. Marcelo Galván Espinoza, Dr. Salvador Cruz Jiménez, Dr. Miguel Castro Martinez y a la Dra. Maria Esther Ruiz Santoyo, por la revisión, comentarios y sugerencias que han hecho a este trabajo. Gracias.

Quiero agradecer a todo el personal del área de Quimica Cuántica de la UAM Iztapalapa, todo el apoyo que me han brindado.

Hago patente mi agradecimiento al Instituto Mexicano del Petróleo, por las facilidades otorgadas para la realización de esta tesis.

También quiero agradecer al Consejo Nacional de Ciencia y Tecnologia el apoyo económico que me ha brindado. 


\section{CONTENIDO}

\section{INTRODUCCION \\ INRODUCCION \\ OBJETIVO \\ CAPITULO 1 \\ CONTAMINACIÓN ATMOSFÉRICA}

Página

1.1 La atmósfera terrestre

1.2 Hidrocarburos y compuestos orgánicos volátiles (COV)

1.3 Generalidades de los óxidos de nitrógeno $\left(\mathrm{NO}_{\mathrm{x}}\right)$

1.4 Esmog fotoquimico

1.5 Quimica y foto-química atmosférica

$1.6 \mathrm{COV}$ en la atmósfera de la Zona Metropolitana de la Ciudad de México 20

1.7 Descripción general de los modelos de calidad del aire

1.7.1 Modelos de caja

1.7.2 Modelos Airshed

1.7.2.1 Modelo Euleriano o de malla

1.7.2.2 Modelo Lagrangiano o de trayectoria

1.8 Reactividad relativa de los COV para formar ozono

\section{CAPITULO 2}

\section{ANTECEDENTES TEORICOS Y EXPERIMENTALES}

2.1 Reacciones atmosféricas de parafinas 31

2.2 Reacciones atmosféricas de compuestos aromáticos 33

2.3 Estudios teóricos para reacciones de parafinas $\quad 37$

2.4 Estudios teóricos de radicales peroxilo $\mathrm{RO}_{2} \bullet$ de las parafinas 42

2.5 Estudios teóricos para reacciones de compuestos aromáticos 43

2.6 Estudios experimentales de compuestos aromáticos 44 


\section{CAPITULO 3}

\section{RESULTADOS Y DISCUSION}

\section{REACCIONES DE OXIDACIÓN DE LAS PARAFINAS}

3.1. Reacción de abstracción de hidrógeno de parafinas por el radical $\mathrm{OH} \bullet \quad 46$

3.2. Reacción de adición de $\mathrm{O}_{2}$ en los radicales $\mathrm{R} \bullet \quad 58$

\section{CAPITULO 4}

\section{RESULTADOS Y DISCUSION}

\section{REACCIONES DE OXIDACIÓN DEL TOLUENO}

4.1 Las reacciones del tolueno con el radical OH• 66

4.1.1 La reacción de abstracción de hidrógeno de tolueno 67

4.1.2 La reacción de adición del radical $\mathrm{OH} \bullet$ al tolueno 69

4.2. La formación de radicales peroxilos aromáticos $\quad 76$

4.2.1 La reacción del radical bencilo con $\mathrm{O}_{2} \quad 76$

4.2.2 La reacción del radical MHCHD con $\mathrm{O}_{2} \quad 78$

4.2.2.1 Radicales peroxilo $\quad 92$

4.2.2.2 Radicales biciclo $\quad 94$

4.2.2.2 Radicales tipo epóxido 95

$\begin{array}{ll}\text { CONCLUSIONES } & 98\end{array}$

$\begin{array}{lr}\text { ANEXO 1 } & 102\end{array}$

$\begin{array}{ll}\text { ANEXO } 2 & 123\end{array}$

$\begin{array}{ll}\text { ANEXO 3 } & 128\end{array}$

$\begin{array}{ll}\text { BIBLIOGRAFIA } & 156\end{array}$ 


\section{INTRODUCCION}

Como consecuencia de los procesos antropogênicos y biogénicos, se emiten grandes cantidades de compuestos orgánicos volátiles (COV) a la atmósfera, las cuales, después de complejas transformaciones fisicas y químicas, generan problemas de contaminación atmosférica, tales como, el esmog fotoquímico, la lluvia ácida, la disminución de la capa de ozono en la estratósfera, asi como modificaciones del clima global.

En diferentes laboratorios del mundo, desde hace más de tres décadas se han realizado una gran cantidad de estudios experimentales y teóricos para entender la fisica y química atmosféricas.

Debido a la complejidad de los procesos atmosféricos, se hace necesario el uso de modelos de calidad del aire basados en códigos computacionales para predecir y evaluar los efectos de las emisiones contaminantes en la atmósfera. Una faceta importante de estos modelos, son los mecanismos de reacción. Se han formulado distintos mecanismos de reacción para explicar la formación del ozono y otros compuestos químicos relacionados con el esmog fotoquímico. En general estos mecanismos contienen todas las reacciones relacionadas con las especies inorgánicas de la atmósfera, y difieren unos de otros en la manera en que se expresan las reacciones de las especies orgánicas. Con el objeto de facilitar los cálculos computacionales se han elaborado mecanismos condensados, en los que se eligen especies prototipo para representar a los diferentes compuestos orgánicos, tales como parafinas, olefinas lineales y ramificadas, compuestos aromáticos, compuestos carbonilo, etc., con lo que el número de reacciones se reduce notablemente. La base de los mecanismos de reacción la constituye el acervo de datos cinéticos y de reacciones de los compuestos individuales.

En la tropósfera, las parafinas y los compuestos aromáticos reaccionan principalmente con el radical hidroxilo $(\mathrm{OH} \bullet)$. No obstante, es bien diferente el conocimiento que se tiene de los mecanismos de reacción de estas dos familias de compuestos. Así, mientras se han determinado con precision la velocidad y los productos de reacción de las parafinas, sobre todo de los compuestos ligeros, se tiene una gran incertidumbre en las velocidades y productos de reacción de los compuestos aromáticos, 
dada la competencia que existe entre los productos que se obtienen a consecuencia del rompimiento del anillo aromático y aquellos que permiten la conservación del mismo.

Como es bien sabido, la atmósfera de la Ciudad de México presenta un grave problema de contaminación ambiental, causada por el esmóg fotoquimico, el cual se forma fácilmente debido a las condiciones atmosféricas y metereológicas que presenta, dicha atmósfera. Debido a que en ella se emiten grandes cantidades de emisiones antropogénicas causadas por aproximadamente 3 millones de automóviles, 300 mil industrias y las ocasionadas por las diversas actividades de los habitantes. Las emisiones más abundantes corresponden principalmente a los compuestos orgánicos volátiles $(\mathrm{COV})$, monóxido de carbono $(\mathrm{CO}), \mathrm{y}$ óxidos de nitrógeno $\left(\mathrm{NO}_{\mathrm{x}}\right.$. También, en dicha atmósfera se presenta una gran cantidad de radiación solar que favorece la reacción entre los COV y los $\mathrm{NO}_{\mathrm{x}}$, formando de esta manera, grandes cantidades de ozono y otros oxidantes fotoquimicos.

Dado que cada compuesto orgánico que se emiten a la atmósfera, presenta un potencial diferente para formar ozono, es necesario estudiar de manera detallada, con base a su reactividad química, cual es el mecanismo de reacción que presenta cada uno de estos compuestos. Una manera adecuada para dilucidar tales mecanismos de reacción, es estudiar la reactividad química de cada compuesto, con base en los diferentes métodos de la quimica cuántica. En efecto, la quimica cuántica es una disciplina que proporciona un marco teórico y conceptual que permite interpretar la mayoria de los fenómenos de interés químico a través de la determinación de la función de onda, definida ésta por la ecuación de Schrödinger, $H \Psi=E \Psi$. No obstante que los fundamentos básicos de esta disciplina fueron desarrollados hace más de cincuenta años, es decir desde los inicios de la mecánica cuántica, su aplicación ha estado en función del desarrollo de computadoras de gran velocidad y capacidad de almacenamiento de información. Los métodos de la química cuántica fueron desarrollados inicialmente para describir propiedades de moléculas aisladas en fase gaseosa, pero actualmente existen modelos que toman en cuenta el efecto del entorno. Con el advenimiento de las estaciones de trabajo y las supercomputadoras, en las últimas décadas la química cuántica ha tenido un desarrollo vertiginoso; la validez de sus postulados ha sido probada en muchos sistemas, y se han determinado propiedades moleculares con una gran precisión, correlacionándolos con datos experimentales.

El tema original del trabajo consistió en la investigación del mecanismo de oxidación atmosférica de los compuestos aromáticos, y en particular del tolueno, ya que 
este compuesto se emite directamente a la atmósfera en la combustión incompleta de los combustibles fósiles. En efecto, en el mecanismo de oxidación atmosférica los compuestos aromáticos, como el tolueno, no esta bien elucidado. Se estima, que del total de carbono reaccionante, se identifica solamente el $50 \%$ en los productos que se cuantifican.

El interés por estudiar la oxidación atmosférica de los compuestos aromáticos se debe a que existe incertidumbre sobre la naturaleza y rendimiento de los productos formados en los diferentes posibles caminos de reacción, a partir de la reacción inicial con el radical $\mathrm{OH} \bullet$. En primer lugar, puede ocurrir ya sea la abstracción de un hidrógeno de la cadena lateral, o la adición directa del radical $\mathrm{OH} \bullet$ al anillo aromático. Además, después de la adición, ocurre una competencia entre las rutas de reacción que favorecen el rompimiento del anillo aromát:co y aquellas que favorecen la conservación del mismo. No se conoce con precisión la distribución de alcoholes aromáticos (fenol, orto-cresol, meta-cresol, para-cresol, etc.) y reacciones subsecuentes de las rutas que permiten la conservación del anillo aromático. Tampoco se conoce cual es la distribución de los productos de reacción que se favorecen, después que el anillo aromático se fragmenta.

Iniciamos con un estudio rápido de las reacciones involucradas en el mecanismo de oxidación del tolueno propuesto por Atkinson [1], Figuras 2.1, 2.2, 2.3 y 2.4 (capitulo 2), utilizando un método semiempírico, el PM3 [2].

A continuación y suponiendo que la reacción de abstracción de hidrógeno del grupo metilo del tolueno es parecida a la de abstracción de un hidrógeno de metano, estudiamos la reacción:

$$
\mathrm{CH}_{4}+\mathrm{OH} \bullet \rightarrow \mathrm{CH}_{3} \bullet+\mathrm{H}_{2} \mathrm{O}
$$

con una variedad de métodos de complejidad creciente [3]. Por el interés que presentó este trabajo, lo ampliamos para estudiar una serie de parafinas [4], tales como el metano, etano, propano, butano, iso-butano y neo-pentano, abstrayendo un hidrógeno primario, secundario y terciario (Capitulo 3). Las principales razones para estudiar con detalle el mecanismo de reacción de las parafinas son las siguientes:

i) El metano es un hidrocarburo poco reactivo para formar ozono, sin embargo, su concentración en la tropósfera es muy alta. Por otra parte, recientemente se ha descubierto que la concentración en la atmósfera de la Ciudad de México, de etano, 
propano, y butano, es anormalmente alta, como consecuencia de la fuga y combustión incompleta del gas LP que se usa como combustible [5].

ii) El acervo de datos experimentales sobre la cinética de las reacciones de oxidación de las parafinas es muy grande y complejo de entender. Asi por ejemplo, mientras se sabe que un radical secundario es más estable que un radical primario, en muchos casos un radical primario favorece más la formación de ozono que un radical secundario o terciario.

iii) De acuerdo a la relación de Arrhenius, $\mathrm{k}=\mathrm{A} \exp \left(-\mathrm{E}_{\mathrm{a}} / \mathrm{T}\right)$, al graficar datos experimentales de constantes de velocidad contra el inverso de la temperatura, para las reacciones de hidrocarburos con el radical $\mathrm{OH} \bullet$, se observa una curva caracteristica que indica que el efecto túnel juega un papel muy importante, en estas reacciones, a temperaturas menores de $500 \mathrm{~K}$.

Paralelamente, la estructura electrónica del radical bencil peroxilo obtenida con PM3 inicialmente, se confirmó con un método ab-initio con correlación electrónica [6], el MP2, con una base 6-31G** (Capitulo 4). Debido a que con esta base y el número de electrones de esta estructura los cálculos requieren una gran cantidad de recursos informáticos y tiempo de CPU, se probó inicialmente con un sistema más pequeño, no saturado, el alil peroxilo [7a].

Una vez que se probaron sistemas moleculares pequeños, como las parafinas y el radical alil peroxilo, se realizó un estudio muy exhaustivo de los aductos del tolueno con el radical $\mathrm{OH} \bullet$ y la subsiguiente adición de oxígeno molecular con el fin de comparar las energias relativas de los múltiples isómeros posibles y proponer el camino más probable de reacción [7b], (Captitulo 4).

En este trabajo reportamos nuevos resultados energéticos y estructurales de los reactivos, estados de transición y productos para las reacciones de oxidación de parafinas y compuestos aromáticos siguientes:

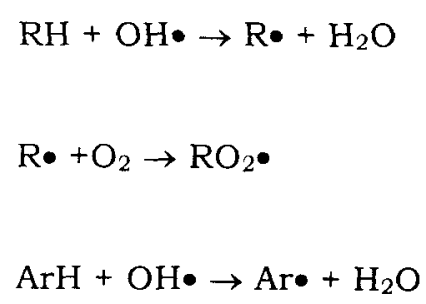




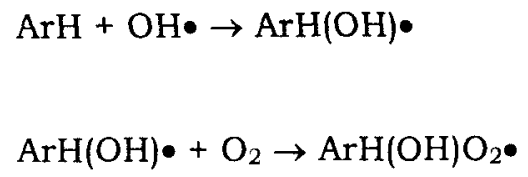

En las dos reacciones, RH puede ser metano, etano, propano, butano, iso-butano y neo-pentano, y $\mathrm{R} \bullet$ es el radical alquilico correspondiente. En las tres últimas reacciones, $\mathrm{ArH}$ es el tolueno, Ar• es el radical aril correspondiente, y $\operatorname{ArH}(\mathrm{OH}) \bullet$ es el radical metil-hidroxi-ciclo-hexa-dienil, (MHCHD).

Para todas estas reacciones, se estudiaron los caminos de reacción tomando en cuenta los detalles de la superficie de energia potencial. Se explicaron, en algunos casos, las energias de activación negativas, como en el caso de las reacciones de adición del $\mathrm{O}_{2}$ al radical $\mathrm{R} \bullet \mathrm{y}$ al radical $\mathrm{ArH}(\mathrm{OH}) \bullet$.

El contenido de la tesis se divide en cuatro capitulos. En el Capitulo 1 se describe de manera general la quimica y fotoquímica de la atmósfera, asi como las causas principales de la contaminación atmosférica. En el Capítulo 2 se presenta una revisión sobre los estudios a nivel teórico y experimental de las reacciones de oxidación de las parafinas y los compuestos orgánicos aromáticos, que la fecha se han realizado. En los Capítulos 3 y 4, se presentan los resultados y la discusión de las reacciones de oxidación de las parafinas (Capitulo 3) y de los compuestos aromáticos (Capitulo 4), obtenidos mediante cálculos de estructura electrónica. Después de estos capitulos se presentan las conclusiones generales del trabajo. Además, se han incluido en la tesis tres Anexos. En el Anexo 1, se hace una breve descripción los principales métodos teóricos utilizados. En el Anexo 2, se describe la metodologia que se usó para la realización de los cálculos estructura electrónica. En el Anexo 3, se reportan las estructuras optimizadas de los estados de transición. 


\section{OBJETIVO}

El objetivo principal de este trabajo es establecer rutas de reacción que ayuden a entender el papel de los compuestos orgánicos volátiles en la química atmosférica y, en particular, en la tropósfera de la Ciudad de México, utilizando cálculos de orbitales moleculares para determinar las estructuras de minima energia de los posibles intermediarios y las superficies de energía potencial de las reacciones. 


\section{CAPITULO 1}

\section{CONTAMINACIÓN ATMOSFÉRICA}

\subsection{La atmósfera terrestre}

La atmósfera terrestre es una capa gaseosa que envuelve a la tierra y está compuesta de una gran variedad de moléculas en fase gas y fase liquida, principalmente de nitrógeno, oxígeno y gases nobles, cuyas concentraciones, en algunos casos, permanecen en la atmósfera por mucho tiempo. La composición del aire limpio en la tropósfera [8], se muestra en la Tabla 1.1

Tabla 1.1 Composición de la atmósfera de la tierra*

\begin{tabular}{|l|l|l|}
\hline Especies & $\begin{array}{c}\text { Concentración promedio } \\
\text { [ppm] }\end{array}$ & \multicolumn{1}{|c|}{$\begin{array}{c}\text { Tiempo aproximado } \\
\text { de residencia }\end{array}$} \\
\hline $\mathrm{N}_{2}$ & 780840 & $10^{6}$ años \\
$\mathrm{O}_{2}$ & 209460 & 10 años \\
$\mathrm{Ar}$ & 9340 & - \\
$\mathrm{CO}_{2}$ & 332 & 15 años \\
$\mathrm{Ne}$ & 18 & - \\
$\mathrm{He}$ & 5.2 & 10 años \\
$\mathrm{CH}$ & 1.65 & 7 años \\
$\mathrm{Kr}$ & 1.1 & - \\
$\mathrm{H}$ & 0.58 & 10 años \\
$\mathrm{N}_{2} \mathrm{O}$ & 0.33 & 10 años \\
$\mathrm{Xe}$ & 0.09 & - \\
$\mathrm{H}_{2} \mathrm{O}$ & $V^{2}$ & 10 dias \\
$\mathrm{SO}_{2}$ & $10^{-5}-10^{-4}$ & 40 dias \\
$\mathrm{NH}_{3}$ & $10^{-4}-10^{-3}$ & 20 dias \\
$\mathrm{NO} \mathrm{NO}_{2}$ & $10^{-6}-10^{-2}$ & 1 dia \\
$\mathrm{O}_{3}$ & $10^{-2}-10^{-1}$ & - \\
$\mathrm{HNO}_{3}$ & $10^{-5}-10^{-3}$ & 1 dia \\
\hline
\end{tabular}

${ }^{*}$ La composición promedio de aire está ordenada de mayor a menor concentración.

Por otra parte, la atmósfera se divide en diferentes regiones [9], basándose en el perfil de temperatura y altitud. En la superficie de la Tierra hay una gran variación de temperatura con la altura. Si se considera el caso de latitudes medias, en las que la temperatura a nivel del mar es de alrededor de $290 \mathrm{~K}$, se observa que a medida que la altitud se incrementa la temperatura disminuye en una proporción de $6.5{ }^{\circ} \mathrm{C} / \mathrm{km}$. La 
región de la atmósfera que se localiza en los primeros $15 \mathrm{~km}$ se conoce como la tropósfera y la región donde la temperatura es minima se conoce como tropopausa. Arriba de ésta se encuentra la estratósfera, una región donde la temperatura se eleva hasta una temperatura máxima de aproximadamente $273 \mathrm{~K}$ a $50 \mathrm{Km}$ (en el ecuador y latitudes medias). La región de máxima temperatura se conoce como estratopausa. Posteriormente, un incremento en la altitud de 50 a $85 \mathrm{Km}$ es acompañado por una disminución de temperatura de 273 a $173 \mathrm{~K}$ a través de la región conocida como mesósfera, hasta alcanzar una temperatura mínima a $85 \mathrm{Km}$ : esta región se conoce como mesopausa. Y finalmente, un incremento en altitud genera un rápido decremento en la temperatura, en una relación de $5{ }^{\circ} \mathrm{C} / \mathrm{Km}$ a $150 \mathrm{Km}$, a través de la región conocida como termósfera, la que se extiende hasta aproximadamente $500 \mathrm{Km}$ de altura.

El perfil de temperatura está relacionado con la composición química de la atmósfera. La disminución de la temperatura con el incremento de la altitud en la tropósfera se debe a que la superficie de la Tierra se calienta por absorción de la radiación visible a longitudes de onda mayores a $300 \mathrm{~nm}$. Además, hay importantes movimientos de corrientes de aire hacia arriba, de tal manera que las particulas y los contaminantes gaseosos del aire pueden ir de la superficie de la Tierra a la parte más alta de la tropósfera en un tiempo de dias o meses, aunque ésto está en función de las condiciones metereológicas de cada región.

Por otro lado, en la estratósfera se llevan a cabo una serie de reacciones fotoquimicas que involucran ozono, $\mathrm{O}_{3}, \mathrm{y}$ las reacciones con oxigeno molecular, $\mathrm{O}_{2}, \mathrm{El} \mathrm{O}_{3}$ absorbe radiación solar en la región de $210-290 \mathrm{~nm}$, mientras que el $\mathrm{O}_{2}$ absorbe a $\leq 200$ nm.

En la mesósfera la disminución de la temperatura se debe a la disminución en la concentración de ozono con la altitud, ya que esto reduce el calor liberado a través de las reacciones de fotodisociación de $\mathrm{O}_{3}, \mathrm{O}_{3}$ con $\mathrm{O}_{2}, \mathrm{y} \mathrm{O}_{2}$ con oxigeno atómico.

En la termósfera la temperatura se eleva debido al aumento de la absorción de la radiación solar por el $\mathrm{O}_{2}$ y el $\mathrm{N}_{2}$, a longitudes de onda menores o iguales a $200 \mathrm{~nm}$, al igual que por algunas especies atómicas. 


\subsection{Hidrocarburos y compuestos orgânicos volátiles (COV)}

Los hidrocarburos, son compuestos formados por átomos de hidrógeno y carbono y se clasifican de acuerdo a su grupo funcional, dentro de los cuales se reconoce la misma estructura básica y difieren por el número de átomos de carbono que contienen. De manera general, se establece que los hidrocarburos de 1 a 4 átomos de carbono se encuentran en fase gas en condiciones estándar de temperatura y presión $\left(25^{\circ} \mathrm{C}\right.$ y 1 atmósfera), mientras que los hidrocarburos con 5 o más átomos de carbono son liquidos o sólidos bajo las mismas condiciones. La mezcla de hidrocarburos en fase gas, observada en atmósferas urbanas contaminadas, por lo general incluye compuestos que, en su forma pura, se encuentran en fase liquida o incluso en fase sólida.

Con base en diferentes estudios, se ha observado que el número de hidrocarburos presentes en la atmósfera es muy grande. En particular, en atmósferas urbanas contaminadas, se han identificado por la técnica acoplada de cromatografia de gases y espectroscopía de masas (GC/MS) cientos de hidrocarburos [10-12], entre los que se incluyen compuestos orgánicos de diferentes familias tales como: parafinas, olefinas, aromáticos, y compuestos orgánicos clorados y oxigenados. Estos últimos requieren, para su medición y análisis, de métodos analíticos como la cromatografia de líquidos de alta presión (HPLC). A todos estos compuestos, en conjunto, se les conoce como compuestos orgánicos volátiles (COV).

La Agencia de Protección Ambiental (EPA) de los Estados Unidos [13], define a los COV como compuestos orgánicos que tienen una presión de vapor mayor de $0.1 \mathrm{mmHg}$ a $25^{\circ} \mathrm{C}$ y que contienen en su estructura molecular desde 2 hasta 14 átomos de carbono. También, se define a los COV como compuestos orgánicos que, al reaccionar con los óxidos de nitrógeno en presencia de la radiación ultravioleta, forman oxidantes fotoquimicos como el ozono, aldehidos, peroxiacetilnitratos, etc. La volatilidad de los hidrocarburos depende del número de átomos de carbono que contengan $\mathrm{y}$ de la temperatura ambiente a la cual están expuestos. El metano es el hidrocarburo más simple y es el hidrocarburo que se encuentra en mayor concentración en la atmósfera, pero su contribución para formar ozono y otros oxidantes fotoquimicos es pequeña por lo que no se considera como un compuesto orgánico volátil. 


\subsection{Generalidades de los óxidos de nitrógeno $\left(\mathrm{NO}_{\mathrm{x}}\right.$ )}

Los óxidos de nitrógeno $\left(\mathrm{NO}_{\mathrm{x}}\right)$, son compuestos que contienen en su estructura molecular átomos de nitrógeno y oxígeno. Son el óxido nítrico (NO), el dióxido de nitrógeno $\left(\mathrm{NO}_{2}\right)$, el óxido nitroso $\left(\mathrm{N}_{2} \mathrm{O}\right)$, el tetraóxido de nitrógeno $\left(\mathrm{N}_{2} \mathrm{O}_{4}\right)$ y el pentóxido de nitrógeno $\left(\mathrm{N}_{2} \mathrm{O}_{5}\right)$. En este grupo tambiên se incluyen otros compuestos tales como, los ácidos nitroso, $\mathrm{HNO}_{2}$, nitrico, $\mathrm{HNO}_{3}$, y peroxinitrico, $\mathrm{HO}_{2} \mathrm{NO}_{2}$. Sin embargo, por su alta concentración en la atmósfera y su gran reactividad química, solo se considera como $\mathrm{NO}_{\mathrm{x}}$ la suma de los dos primeros óxidos de nitrógeno [14], debido a que ellos constituyen más del $98 \%$ de esta mezcla. La principal fuente de emisión de los $\mathrm{NO}_{\mathrm{x}}$ en atmósferas urbanas y limpias son los procesos de combustión incompleta de combustibles fósiles, ampliamente utilizados en automóviles, industrias, centrales termoeléctricas, sistemas de calentamiento residencial, etc. El NO es la especie dominante producida en los procesos de combustión, constituye aproximadamente el $95 \%$ del total de $\mathrm{NO}_{\mathrm{x}}$. Esto es consecuencia del uso desmedido de combustibles fósiles y del desgaste de las máquinas, que favorece procesos de combustión incompleta.

Si la combustion fuera completa únicamente se formaria $\mathrm{CO}_{2}$ y el $\mathrm{H}_{2} \mathrm{O}$, de acuerdo a la reacción (1.1):

$$
\mathrm{HC}+\mathrm{O}_{2} \rightarrow \mathrm{CO}_{2}+\mathrm{H}_{2} \mathrm{O}
$$

\subsection{Esmog fotoquimico}

La palabra esmog se deriva de smoke (humo) y fog (niebla). El esmog fotoquímico se refiere a la formación de oxidantes fotoquimicos producidos por las reacciones quimicas entre los hidrocarburos y los $\mathrm{NO}_{\mathrm{x}}$ iniciadas con la radiación solar, en la troposfera. El proceso de producción de ozono involucra cientos de reacciones quimicas que ocurren simultáneamente. El esmog fotoquimico fue observado por primera vez en 1944 [15], en la Ciudad de Los Angeles, EUA. Este esmog es diferente al observado en Londres, Inglaterra, a finales de 1952, el cual se caracterizó por el alto contenido de $\mathrm{SO}_{2}$ y materia particulada [16]. En los años recientes, otras ciudades con una gran densidad de población como México D. F., Denver, Tokio, Roma y Atenas presentan grandes problemas de contaminación ambiental por ozono [17]. 
La formación del esmog fotoquímico en la tropósfera se inicia con la fotólisis del $\mathrm{NO}_{2}$ en presencia de radiación ultravioleta en el intervalo de 300 a $420 \mathrm{~nm}$. La fotodisociación del $\mathrm{NO}_{2}$ es muy rápida, en condiciones de radiación ultravioleta con un ángulo zenital de $40^{\circ}$, por lo que la concentración de $\mathrm{NO}_{2}$ se reduce a la mitad en menos de dos minutos. En la troposfera esta reacción se lleva a cabo por medio del ciclo fotoestacionario de $\mathrm{NO}_{2}$ :

$$
\begin{aligned}
& \mathrm{NO}_{2}+\mathrm{h} v(\lambda=420 \mathrm{~nm}) \rightarrow \mathrm{NO}+\mathrm{O}\left({ }^{3} \mathrm{P}\right) \\
& \mathrm{O}\left({ }^{3} \mathrm{P}\right)+\mathrm{O}_{2}+\mathrm{M} \rightarrow \mathrm{O}_{3} \\
& \mathrm{O}_{3}+\mathrm{NO} \rightarrow \mathrm{NO}_{2}+\mathrm{O}_{2}
\end{aligned}
$$

donde $\mathrm{M}$ es una molécula que favorece los choques por colisión entre las moléculas.

Como se observa, la fotólisis del $\mathrm{NO}_{2}$ produce oxigeno atómico en su estado fundamental, el triplete ${ }^{3} \mathrm{P}$, que al reaccionar con $\mathrm{O}_{2}$ constituye el mecanismo principal para la producción de $\mathrm{O}_{3}$ en la tropósfera. En atmósferas urbanas contaminadas, la alta concentración de ozono se utiliza como indicador de la contaminación del aire por esmog fotoquimico. Una fracción del $\mathrm{NO}$ emitido reacciona con el $\mathrm{O}_{3}$ para generar $\mathrm{NO}_{2}$ y esta última reacción cierra el ciclo fotoestacionario de $\mathrm{NO}, \mathrm{NO}_{2} \mathrm{y} \mathrm{O}_{3}$.

En atmósferas que contienen únicamente $\mathrm{NO}$ y $\mathrm{NO}_{2}$, las reacciones que controlan las concentraciones de estos contaminantes son las reacciones que van de la (1.2) a la (1.4), anteriormente establecidas. Estas reacciones representan un patrón cíclico iniciado por la radiación solar. Durante el dia, el flujo de radiación solar cambia con el movimiento del sol. No obstante, si se consideran periodos de tiempo cortos $(\sim 10$ minutos), el flujo puede ser considerado constante, en cuyo caso la velocidad de la reacción (1.2), puede ser expresada como:

$$
\text { velocidad }=\mathrm{k}_{1.2}\left[\mathrm{NO}_{2}\right]
$$

donde $k_{2.2}$ es una función de la hora del dia. Si se considera la cinética de un sistema en el que las reacciones (1.2), (1.3) y (1.4) son las únicas que tienen lugar, y bajo la suposición de que se conocen las concentraciones iniciales de $\mathrm{NO}$ y $\mathrm{NO}_{2}$, en un reactor de volumen y temperatura constantes, se tiene que:

$$
\mathrm{d}\left[\mathrm{NO}_{2}\right] / \mathrm{dt}=-\mathrm{k}_{2.2}\left[\mathrm{NO}_{2}\right]+\mathrm{k}_{2.4}\left[\mathrm{O}_{3}\right][\mathrm{NO}]
$$


donde $\mathrm{k}_{1.2}$ corresponde a la velocidad de reacción del $\mathrm{O}_{3}$ de acuerdo a la ecuación (1.4).

Considerando la concentración de $\mathrm{O}_{2}$ como constante, hay cuatro especies en el sistema: $\mathrm{NO}_{2}, \mathrm{NO}, \mathrm{O}$ y $\mathrm{O}_{3}$. Para cada una de estas especies se pueden escribir sus ecuaciones cinéticas. Si $\mathrm{k}_{2.3}$ es la velocidad de reacciōn asociada a la ecuación (1.3), para la concentración de oxigeno, se tiene:

$$
\mathrm{d}[\mathrm{O}] / \mathrm{dt}=\mathrm{k}_{2.2}\left[\mathrm{NO}_{2}\right]-\mathrm{k}_{2.3}[\mathrm{O}]\left[\mathrm{O}_{2}\right][\mathrm{M}]
$$

El oxigeno atómico es tan reactivo que desaparece en la reacción (1.3) tan rápido como se forma en la reacción (1.2). De esta manera es posible utilizar la aproximación del estado pseudo estable, por lo que la velocidad de formación es exactamente igual a la velocidad de desaparición:

$$
\mathrm{K}_{1.2}\left[\mathrm{NO}_{2}\right]=-\mathrm{k}_{1.3}[\mathrm{O}]\left[\mathrm{O}_{2}\right][\mathrm{M}]
$$

y la concentración de $\mathrm{O}$ en el estado estacionario, se expresa como:

$$
[\mathrm{O}]=\mathrm{k}_{1.2}\left[\mathrm{NO}_{2}\right] / \mathrm{k}_{1.3}\left[\mathrm{O}_{2}\right][\mathrm{M}]
$$

La concentración de $\mathrm{O}_{3}$ er estado fotoestacionario es:

$$
\left[\mathrm{O}_{3}\right]=\mathrm{k}_{1.2}\left[\mathrm{NO}_{2}\right] / \mathrm{k}_{1.4}[\mathrm{NO}]
$$

De acuerdo a la última ecuación se observa que la concentración de $\left[\mathrm{O}_{3}\right]$ depende de la relación $\left[\mathrm{NO}_{2}\right] /[\mathrm{NO}]$ para cualquier valor de $\mathrm{k}_{2.2}$. El valor máximo de $\mathrm{k}_{2.2}$ depende de la latitud, época del año y hora del dia.

\subsection{Quimica y foto-quimica atmosférica}

La energia de un fotón para las regiones ultravioleta (UV) y visible (VIS), es del orden de magnitud de los calores de formación o cambios de entalpias de las reacciones químicas incluyendo aquellas en que una molécula diatómica se disocia en átomos. Para la disociación del oxigeno molecular, se necesita un cambio de entalpia de $495 \mathrm{KJ} \mathrm{mol}^{-1}$, de acuerdo a la reacción (1.11): 
Cuando una reacción se inicia por medio de la radiación, se dice que ésta es una reacción fotoquímica. En el caso anterior, el oxigeno molecular sufre una descomposición fotoquímica a través de una reacción de fotólisis. Las moléculas que absorben luz no retienen más de $10^{-12} \mathrm{~s}$ el exceso de energia que les concede un fotón, dado que en una pequeña fracción de segundo pueden reaccionar fotoquimicamente o disipar esta energia a través de colisiones o choques con otras moléculas [17]. Muchas de las reacciones que se llevan a cabo en la tropósfera se inician por reacciones fotolíticas.

Con radiación ultravioleta, los contaminantes atmosféricos se fotolizan y fragmentan produciendo átomos, iones y radicales libres, o bien en algunos casos sólo se excitan cambiando su reactividad química. La atmósfera actúa como un filtro de radiación de onda corta, ya que las especies que la constituyen absorben radiación en onda corta. La fotoquímica de la troposfera esta dominada por especies como: $\mathrm{O}_{3}, \mathrm{NO}_{2}$, $\mathrm{SO}_{2}$ y formaldehido, $\mathrm{HCHO}$, las cuales absorben radiación ultravioleta.

\section{$1.6 \mathrm{COV}$ en la atmósfera de la Zona Metropolitana de la Ciudad de México (ZMCM)}

La ZMCM, abarca una superficie de $2346 \mathrm{~km}^{2}$ y en ella habitan aproximadamente 18 millones de personas. Todos los dias se vierten a la atmósfera las emisiones de aproximadamente 3 millones de automóviles, 300 mil industrias y las ocasionadas por las diversas actividades de los habitantes. Las emisiones más abundantes corresponden a procesos de combustión incompleta que generan principalmente monóxido de carbono (CO), óxidos de nitrógeno $\left(\mathrm{NO}_{\mathrm{x}}\right.$ y los compuestos orgánicos volátiles. Estos últimos llegan también a la atmósfera a través de emisiones biogénicas y por evaporación de combustibles y disolventes.

En la atmósfera de la Ciudad de México las concentraciones atmosféricas de CO, $\mathrm{O}_{3}$ y $\mathrm{NO}_{\mathrm{x}}$ son indicadores de la calidad del aire, por ello en la ZMCM se han medido desde 1986 mediante la Red Automática de Monitoreo Atmosférico (RAMA) que actualmente administra el Gobierno de la Ciudad. A pesar de que los COV son precursores en la formación del ozono, no es fácil establecer un criterio que relacione su concentración en la atmósfera con la formación de ozono, debido a que cada especie individual presenta una reactividad quimica atmosférica diferente. Sin embargo, la determinación tanto de las concentraciones totales de COV como la concentración de las especies individuales es 
de gran utilidad para la planeación de estrategias de control de emisiones de COV de diferentes fuentes de emisión.

La red automática de monitoreo atmosférico cuenta con 25 estaciones distribuidas estratégicamente en la $\mathrm{ZMCM}$ [18]. En esta red de monitoreo se miden ozono, monóxido de carbono, dióxido de nitrógeno, dióxido de asufre, hidrocarburos no metánicos, y ciertos parámetros metereológicos: velocidad y dirección del viento, humedad relativa $\mathrm{y}$ temperatura (Tabla 1.2). Sin embargo, no todos los contaminantes y parámetros metereológicos se miden en todas y cada una de las 25 estaciones. Así, el $\mathrm{SO}_{2}$ es el contaminante que más se mide (17 estaciones), mientras que el $\mathrm{H}_{2} \mathrm{~S}$ solo se mide en 2 estaciones. $\mathrm{El} \mathrm{O}_{3}, \mathrm{CO}, \mathrm{NO}_{2}$ y $\mathrm{NO}_{\mathrm{x}}$ se miden en 12, 15, 11 y 11 estaciones respectivamente. Cabe señalar, que aunque los hidrocarburos son precursores importantes de ozono, como se ha establecido anteriormente, éste es uno de los contaminantes que menos se mide, ya que solo se hace en tres estaciones (Tlalnepantla, La Merced y Pedregal).

Además de esta red que mide ozono y sus precursores, existe la red manual de monitoreo atmosférico que consta de 19 estaciones (Tabla 1.3) y mide de manera intermitente la concentración de partículas suspendidas totales (PST), y la concentración de particulas cuyo diámetro es menor a $10 \mu \mathrm{m}\left(\mathrm{PM}_{10}\right)$.

Los datos que se obtienen de la red manual complementan las mediciones de la red automática de monitoreo, aunque la frecuencia de medición es menor (una muestra cada seis dias aproximadamente), debido a que la técnica de muestreo implica dedicar mayor tiempo de trabajo de laboratorio. Cabe señalar que la red manual, además de medir la concentración de PST y PM10, también puede medir la concentración de plomo, cobre, fierro, cadmio, niquel, cobalto, zinc, nitratos y sulfatos. En la red manual de monitoreo, es posible realizar muestreos programados de otros contaminantes atmosféricos, como los aldehídos, para los que no existe una metodologia continua de monitoreo. La Tabla 1.4 describe la metodologia de muestreo y análisis de los contaminantes medidos por la RAMA.

Para efectos de control y garantia de calidad del sistema de mediación de la RAMA, esta cuenta con un laboratorio de calibración y certificación único en América Latina, en el que sus equipos de medición son sometidos a pruebas de funcionalidad, eficiencia y confiabilidad, a través de un programa de control y garantía de calidad coordinado entre el gobierno de la Ciudad de México y la EPA. No obstante, la red de monitoreo ambiental 
que opera en la Ciudad de México dificilmente cumple con los requerimientos mínimos para generar información necesaria para un modelo de dispersión tridimensional que simule la evolución temporal y espacial de contaminantes fotoquimicos. Por esta razón, desde 1992 a la fecha, el Instituto Mexicano del Petróleo (IMP) junto con la EPA y las autoridades ambientales en México han realizado diferentes campañas de medición de hidrocarburos en el aire, recolectando muestras en canisteres o contenedores metálicos, siguiendo la metodología TO14 de la EPA [13]. Las campañas de monitoreo se han realizado en el mes de marzo, debido a que en este mes es cuando se presentan los más graves episodios de ozono. También se han realizado muestreos adicionales en el mes de noviembre con objeto de comparar los resultados entre las diferentes estaciones del año (Tabla 1.5).

Tabla 1.2 Estaciones de monitoreo ambiental de ozono y sus precursores.

\begin{tabular}{|c|c|c|c|c|c|c|c|c|c|c|c|c|}
\hline No & ${ }^{2} \mathrm{Ca}$. & Localización & Zona & $\mathbf{S O}_{2}$ & $\mathrm{O}_{3}$ & $\mathrm{CO}$ & $\mathrm{NO}_{2}$ & NO $_{x}$ & $\mathbf{H}_{2} \mathbf{S}$ & bHCNM & cMe. & Total \\
\hline 1 & $Z$ & Lagunilla & $\bar{C}$ & & $\mathrm{X}$ & $X$ & & & & & & 2 \\
\hline 2 & $E$ & Vallejo & NO & $\mathrm{X}$ & & & & & & & & 1 \\
\hline 3 & $\mathrm{~S}$ & Sta. Ursula & $\mathrm{SO}$ & $\mathrm{X}$ & & & & & & & & 1 \\
\hline 4 & $\mathrm{~B}$ & Tacuba & NO & $\mathrm{X}$ & & & $\mathrm{X}$ & $\mathrm{X}$ & $\mathrm{X}$ & & $\mathrm{X}$ & 5 \\
\hline 5 & G & ENEP-Acat & NO & $\mathrm{X}$ & & & $\mathrm{X}$ & $\mathrm{X}$ & & & $\mathrm{X}$ & 4 \\
\hline 6 & M & Los Laureles & $\mathrm{NE}$ & $\mathrm{X}$ & & & & & & & & 1 \\
\hline 7 & $\mathrm{H}$ & La Presa & $\mathrm{NE}$ & $\mathrm{X}$ & & & & & & & & 1 \\
\hline 8 & $J$ & La Villa & $\mathrm{NE}$ & $X$ & & & & & & & & 1 \\
\hline 9 & $\mathrm{~N}$ & San Agustín & $\mathrm{NE}$ & $\mathrm{X}$ & $\mathrm{X}$ & & $\mathrm{X}$ & $\mathrm{X}$ & & & $\mathrm{X}$ & 5 \\
\hline 10 & $\mathrm{C}$ & Azcapotzalco & NO & $\mathrm{X}$ & $X$ & & & & & & & 2 \\
\hline 11 & $\mathrm{~F}$ & Tlalnepantla & NO & $\mathrm{X}$ & $\mathrm{X}$ & $\mathrm{X}$ & $\mathrm{X}$ & $\mathrm{X}$ & & $\mathrm{X}$ & $X$ & 7 \\
\hline 12 & $\mathrm{~L}$ & Xalostoc & NE & $\mathrm{X}$ & $\mathrm{X}$ & $\mathrm{X}$ & $\mathrm{X}$ & $\mathrm{X}$ & & & $\mathrm{X}$ & 7 \\
\hline 13 & $X$ & La Merced & $\mathrm{C}$ & $\mathrm{X}$ & $\mathrm{X}$ & $\mathrm{X}$ & $\mathrm{X}$ & $\mathrm{X}$ & $\mathrm{X}$ & $\mathrm{X}$ & $\mathrm{X}$ & 8 \\
\hline 14 & $\mathrm{~T}$ & Pedregal & SO & $\mathrm{X}$ & $\mathrm{X}$ & $\mathrm{X}$ & $\mathrm{X}$ & $\mathrm{X}$ & & $X$ & $\mathrm{X}$ & 7 \\
\hline 15 & Q & C. Estrella & SE & $\mathrm{X}$ & $\mathrm{X}$ & $\mathrm{X}$ & $\mathrm{X}$ & $\mathrm{X}$ & & & $\mathrm{X}$ & 7 \\
\hline 16 & $U$ & Plateros & SO & & $\mathrm{X}$ & $\mathrm{X}$ & $\mathrm{X}$ & $\mathrm{X}$ & & & $\mathrm{X}$ & 5 \\
\hline 17 & $\mathrm{Y}$ & Hangares & $\mathrm{C}$ & $\mathrm{X}$ & $\mathrm{X}$ & & $\mathrm{X}$ & $\mathrm{X}$ & & & $\mathrm{X}$ & 6 \\
\hline 18 & $\mathrm{P}$ & UAM-Izt. & $\mathrm{SE}$ & & $\mathrm{X}$ & $\mathrm{X}$ & & & & & & 2 \\
\hline 19 & $\mathrm{~K}$ & Aragón & $\mathrm{NE}$ & $\mathrm{X}$ & & $\mathrm{X}$ & & & & & & 2 \\
\hline 20 & 0 & Neza & $\mathrm{NE}$ & $\mathrm{X}$ & & $\mathrm{X}$ & & & & & & 2 \\
\hline 21 & D & I.M.P. & NO & & & $\mathrm{X}$ & & & & & & 1 \\
\hline 22 & $\mathrm{~W}$ & B. Júarez & C & & & $\mathrm{X}$ & $\mathrm{X}$ & $\mathrm{X}$ & & & & 3 \\
\hline 23 & $\mathrm{R}$ & Taxqueña & $\mathrm{SE}$ & & $\mathrm{X}$ & $\mathrm{X}$ & & & & & & 2 \\
\hline 24 & V & Insurgentes & C & & & $X$ & & & & & & 1 \\
\hline 25 & A & Cuitláhuac & NO & 17 & 12 & $\begin{array}{l}X \\
15\end{array}$ & 11 & 11 & 2 & 3 & 10 & 1 \\
\hline
\end{tabular}

a Caseta, ${ }^{\mathrm{b}}$ Hidrocarburos no metánicos, c Parámetros Metereológicos

Como se observa en la Tabla 1.5, la concentración promedio de COV en la ZMCM es alta. Cabe señalar que, estas concentraciones varian de acuerdo a la época del año. Asi, en primavera (mes de marzo) se observan las concentraciones más altas mientras que 
en otoño (mes de noviembre) estas disminuyen. Una razón podria ser, el cambio de combustible que se usa en estas dos diferentes épocas de año.

Tabla 1.3 Estaciones de monitoreo ambiental para particulas supendidas.

\begin{tabular}{|l|l|l|l|l|l|l|}
\hline No & Caseta & Localización & Zona & PST & PM 10 & Total \\
\hline 1 & PO & Portales & CE & & & \\
2 & PD & Pedregal & SO & $X$ & $X$ & 2 \\
3 & FA & Felipe Angeles & SO & & & \\
4 & TX & Taxqueña & SE & & & \\
5 & ES & Cerro de la Estrella & SE & & $X$ & 2 \\
6 & VC & Vicentina & SE & & $X$ & \\
7 & NT & Neza & NE & $X$ & & 1 \\
8 & AP & Aeropuerto & SE & & & \\
9 & VER & Fray Bernardino & CE & & & \\
10 & MU & Museo de la Ciudad de México & SE & $X$ & $X$ & 2 \\
11 & MC & La Merced & SO & & & \\
12 & CFE & Museo Tecnologico & SO & & & \\
13 & LO & Lomas & NO & & & \\
14 & SH & Secretaria de Hacienda & NO & $X$ & $X$ & 2 \\
15 & TL & Tlanepantla & NE & & $X$ & 1 \\
16 & VI & La Villa & NE & $X$ & $X$ & 2 \\
17 & XA & Xalostoc & NE & $X$ & & 1 \\
18 & LP & La Presa & NE & & & \\
19 & CHA & Chapingo & & & & \\
\hline
\end{tabular}

Tabla 1.4 Parámetros y equipos de monitoreo utilizados por la RAMA

\begin{tabular}{|c|c|}
\hline Parámetro & Equipo de monitoreo \\
\hline Partículas & Dispersión Lumínica \\
\hline $\mathrm{SO}_{2}$ & Fluorescencia UV pulsante \\
\hline $\mathrm{CO}$ & Absorción IR con filtro gaseoso de correlación \\
\hline $\mathrm{O}_{3}$ & Fotometria ultravioleta \\
\hline Oxidos de Nitrógeno & Quimiluminiscencia en fase gaseosa \\
\hline $\mathrm{H}_{2} \mathrm{~S}$ & Fluorescencia UV pulsante \\
\hline HCNM & Fotoionización ultravioleta \\
\hline $\begin{array}{l}\text { Humedad relativa } y \\
\text { temperatura }\end{array}$ & Capacitor de película delgada de polímero/termisor \\
\hline $\begin{array}{l}\text { Velocidad y dirección } \\
\text { del viento }\end{array}$ & Veleta y anemómetro convencional \\
\hline
\end{tabular}

En términos generales se observa que, la concentración promedio de parafinas es muy alto, del orden de $2 \mathrm{ppbC}$ (partes por billón de Carbono), mientras que la concentración de compuestos oxigenados y halogenados es baja. La concentración de compuestos aromáticos y alquenos es parecida y oscila entre 0.4 y $0.8 \mathrm{ppbC}$. 
La concentración de compuestos desconocidos es de alrededor de $0.2 \mathrm{ppbC}$. Por cierto, estos compuestos se refieren a compuestos orgánicos no identificados por las técnicas de análisis empleadas en el protocolo T014 de la EPA, la cromatografia de gases acoplada a la espectroscopía de masas (GC/MS).

Tabla 1.5 Concentración de COV en ppbC medidos de 6:00 a 9:900 horas en la ZMCMa.

\begin{tabular}{|l|l|l|l|l|l|l|l|}
\hline \multicolumn{1}{|c|}{ GRUPO } & Mar-92 & Mar-93 & Nov-93 & Nov-94 & Mar-95 & \multicolumn{1}{|c|}{ Nov-95 } & \multicolumn{1}{c|}{ Mar-96 } \\
\hline Alcanos & 1.997 & 2.764 & 2.512 & 1.74 & 1.736 & 1.524 & 1.875 \\
Aromáticos & 0.614 & 0.754 & 0.815 & 0.557 & 0.437 & 0.48 & 0.568 \\
Alquenos & 0.409 & 0.448 & 0.448 & 0.322 & 0.319 & 0.33 & 0.394 \\
Desconocidos & 0.22 & 0.318 & 0.215 & 0.271 & 0.316 & 0.225 & 0.315 \\
Acetileno & 0.113 & 0.169 & 0.133 & 0.112 & 0.137 & 0.133 & 0.15 \\
Oxigenados & 0.049 & 0.091 & 0.063 & 0.061 & 0.054 & 0.038 & 0.043 \\
Halogenados & & 0.025 & 0.043 & 0.059 & 0.04 & 0.028 & 0.041 \\
COV Totales & 3.402 & 4.569 & 4.229 & 3.122 & 3.039 & 2.758 & 3.386 \\
\hline
\end{tabular}

aFuente: Laboratorio de química ambiental del IMP

\subsection{Descripción general de los modelos de calidad del aire}

Los modelos de calidad del aire, se basan en una descripción matemática de la difusión, dispersión y transformación de los contaminantes [19,25]. En general, los modelos de calidad del aire se clasifican en dos tipos: fisicos y matemáticos.

Los modelos físicos simulan los procesos fisicos y quimicos que sufren los contaminantes por medio de una representación, a escala, del problema de contaminación atmosférica. Estos modelos pueden hacer uso de experimentos en túneles de viento o bien de experimentos en cámaras de esmog. Estos últimos han sido ampliamente utilizados como medio para estudiar aisladamente diferentes procesos quimicos atmosféricos.

Una cámara de esmog, es un reactor quimico en el que se introducen diversos contaminantes primarios en concentraciones típicas de la atmósfera y se deja que reaccionen bajo estas condiciones de temperatura, y presión. Cabe señalar que, en los experimentos realizados en cámaras de esmog no se pueden simular la variabilidad, transporte o difusión atmosférica de las emisiones contaminantes. Es decir, en un modelo fisico, no es posible relacionar directamente las emisiones con la calidad del aire y 
considerar simultáneamente una extensa gama de condiciones meteorológicas y de fuentes de emisión como las existentes en una zona urbana.

Los modelos matemáticos, se dividen en dos grupos: a) modelos basados en el análisis estadistico de datos obtenidos por una red de monitoreo atmosférico; y b) modelos basados en la descripción fundamental de transporte y de los procesos quimicos atmosféricos. Los modelos matemáticos simulan los procesos atmosféricos relacionados con la contaminación del aire, y se basan en las ecuaciones de conservación de masa de cada contaminante. Para su desarrollo, estos modelos requieren información sobre factores de emisión, meteorologia, quimica del aire, asi como información de los procesos de eliminación de los contaminantes.

Otro tipo de modelos, que se han desarrollado para el control del ozono y otros oxidantes fotoquímicos (contaminantes secundarios), son los modelos fotoquimicos. Estos modelos describen la relación entre las emisiones y concentración de contaminantes secundarios producidos por reacciones químicas en el aire durante el transporte, la dispersión y la acumulación de los contaminantes primarios. Estos modelos, también denominados modelos de simulación de calidad del aire, son descripciones matemáticas del transporte atmosférico, de la difusión y las reacciones quimicas que sufren los contaminantes.

Existen diferentes tipos de modelos fotoquimicos, entre los que se encuentran, los modelos de caja y los modelos airshed, los cuales se describen a continuación.

\subsubsection{Modelos de caja}

Estos modelos consideran la región a modelar como una sola celda, o caja, limitada en la parte baja por el piso y en la parte alta por una inversión térmica u otro limite superior de mezclado. A los lados, se limitan por las dimensiones del área a modelar. Dentro de la caja, las emisiones son vertidas por diversas fuentes y se supone que el mezclado de tales emisiones es instantáneo y uniforme. En este tipo de modelos es fundamental la suposición de que las emisiones son espacialmente homogéneas e instantáneamente mezcladas. Bajo esta suposición, la concentración de contaminantes se describe mediante un balance de materia que considera la rapidez con la cual son transportados los contaminantes hacia adentro y hacia fuera del volumen de aire, la rapidez con la cual se emiten los contaminantes por las fuentes, la velocidad con la cual 
el volumen se expande y se contrae (en la vertical), la rapidez con la que entran y salen contaminantes por el techo de la caja, asi como la rapidez con la cual los contaminantes reaccionan en la atmósfera o bien se depositan en la superficie.

Por su formulación, los modelos de caja pueden predecir sólo las variaciones temporales de las concentraciones regionales promedio para cada tipo de contaminante. Estos modelos carecen de resolución espacial y no pueden utilizarse en situaciones donde los modelos meteorológicos o de emisiones varian significativamente a través de la región modelada. Cabe señalar que los efectos combinados de patrones de emisiones locales y condiciones meteorológicas, normalmente generan variaciones espaciales significativas en la concentración de los contaminantes estimadas por los modelos.

\subsubsection{Modelos airshed}

A diferencia de los modelos de caja, los modelos airshed toman en cuenta variaciones temporales, es decir, los modelos de columna o celda de aire (airshed) incorporan descripciones realistas de las emisiones, la meteorologia, la química y los sumideros de los contaminantes presentes en un escenario a modelar. Existen dos tipos de estos modelos: el modelo Euleriano y el modelo Lagrangiano.

\subsubsection{Modelo Euleriano o de malla}

Este modelo, se ha desarrollado para una área geográfica fija. Las concentraciones de los contaminantes se calculan en diferentes tiempos. El área geográfica puede abarcar un kilómetro cuadrado o más. Las concentraciones de los contaminantes se estiman a partir de las concentraciones iniciales de los contaminantes dentro del área donde se realiza la modelación, de las emisiones de los contaminantes, del transporte de los contaminantes dentro y fuera de la malla.

La aplicación de estos modelos permite estimar las concentraciones de los contaminantes, para diferentes localidades ubicadas en la región donde se efectúa la modelación, como una función del tiempo. Otra ventaja de estos modelos es la resolución geográfica que poseen; es decir, dado un control de las fuentes emisoras de intensidad parecida, pero localizadas en diferentes sitios, pueden apreciarse mejor los cambios en los efectos que tienen éstas emisiones. 
La base de los modelos de malla es la ecuación de difusión atmosférica, que expresa la conservación de la masa de cada contaminante en un fluido turbulento en el cual ocurren las reacciones quimicas. Una de las ventajas de los modelos de malla, es que éstos son un medio para predecir la distribución tridimensional de las concentraciones de contaminantes, para una región dada. No obstante, sus limitaciones son derivadas principalmente de los datos de entrada. Cuando se cuenta con datos adecuados, los modelos de malla proporcionan una buena aproximación a la calidad del aire.

Básicamente, los modelos fotoquimicos difieren principalmente en el número de procesos atmosféricos considerados en éstos, el nivel de sofisticación en el tratamiento de tales procesos, así como en los métodos numéricos usados para resolver sistemas de ecuaciones.

\subsubsection{Modelo Lagrangiano o de trayectoria}

Los modelos de trayectoria están basados en la ecuación de difusión atmosférica, que describe el transporte de contaminantes. En estos modelos se define una columna de aire hipotética, limitada en la base por el piso y en su parte superior por la base de una inversión térmica o bien por otro límite establecido. Al inicio, la columna se desplaza bajo la influencia de los vientos prevalecientes sobre las fuentes de emisión con lo cual son incorporados los contaminantes primarios a la columna, y posteriormente se simulan las reacciones quimicas dentro la columna. A su vez, la columna puede dividirse en varias capas de aire, suponiéndose en este caso que se presenta un mezclado de las celdas inferiores hacia las celdas superiores. En los modelos de trayectoria de una sola capa, comúnmente se supone un mezclado homogéneo e instantáneo.

Otra suposición que se hace en éstos modelos es que la columna de aire retiene su forma vertical al desplazarse por la acción del viento. Esta consideración, supone una idealización del flujo de vientos, en la cual la velocidad promedio de éstos no cambia a diferentes alturas. Asimismo, algunos modelos de trayectoria suponen que las dimensiones laterales de la columna no varian y tampoco se ven afectadas por la convergencia y divergencia del campo de vientos.

Algunos modelos de trayectoria que sólo consideran una columna de aire, no toman en cuenta la difusión horizontal de los contaminantes. Esto no tiene 
repercusiones importantes en los resultados si las fuentes de emisión están idealmente distribuidas de manera uniforme, de no ser asi, a menos que la columna de aire pase durante su recorrido por grandes fuentes emisoras, el no considerar el efecto de difusión atmosférica puede ocasionar que se atenúe o elimine el efecto de fuentes de emisión fijas. Es decir, en caso de existir un gradiente de concentraciones significativo, pueden obtenerse resultados pobres al despreciarse la dilución horizontal.

En cuanto a las limitaciones del modelo de trayectoria, éste no puede aplicarse en áreas rugosas o terrenos complejos donde los vientós tienen una componente vertical significativa y una considerable variación en los componentes horizontales con la altura de mezcla.

\subsection{Reactividad relativa de los COV para formar ozono}

Como se ha establecido anteriormente, el ozono no se emite a la atmósfera, se forma a través de una reacción fotoquímica entre los compuestos orgánicos volátiles y los óxidos de nitrógeno. Dado que, a la atmósfera se emiten diferentes tipos de compuestos orgánicos, cada uno de ellos presenta una constante de velocidad diferente y por lo tanto un potencial diferente para formar ozono ${ }^{[20-21] . ~}$

Así, se tiene que la reactividad de los COV se define como la fracción de un COV determinado que sufre una reacción quimica durante un episodio de contaminación ambiental. Generalmente esta reactividad depende de la velocidad con que los COV reaccionan en la atmósfera y de las condiciones mismas del episodio, tales como, la intensidad de la radiación y los niveles de concentración de los radicales libres $\mathrm{OH} \bullet$, R•, $-\mathrm{NO}_{3}$. No obstante, como los $\mathrm{COV}$ reaccionan únicamente con el radical $\mathrm{OH} \bullet$, la reactividad cinética está en función solamente de la constante de velocidad de reacción $\mathrm{k}_{\mathrm{OH}}$ y es característica del escenario o episodio de contaminación ambiental. Por lo tanto, si se conoce la dependencia de la $\mathrm{k}_{\mathrm{OH}}$ para un escenario determinado, se puede conocer la reactividad cinética de cualquier COV [22].

Existe un criterio más relevante, que es el cambio en la concentración de ozono causado por una variación de concentración de las emisiones de COV dentro del escenario de contaminación ambiental. Razón por la cual se han derivado diferentes escalas de reactividad de COV para formar ozono. Entre las escalas más importantes, obtenidas a partir de escenarios de contaminación ambiental y experimentos en cámaras 
de esmog o de radiación, se tienen: la escala de reactividad en función de la constante de velocidad $\mathrm{k}_{\mathrm{OH}}$, y la escala de reactividad incremental máxima o MIR.

La escala $\mathrm{OH}$ en un escenario de contaminación ambiental, se define a través de relación de ajuste [20-21],

$$
R_{\mathrm{OH}}\left(k_{\mathrm{OH}}\right)=\left(1-\exp \left(-k_{\mathrm{OH}}\right)^{I N T O H}\right)
$$

donde $\mathrm{R}_{\mathrm{OH}}$ es la reactividad y INTOH es un parámetro que depende solamente del escenario y refleja principalmente el nivel de concentración del radical $\mathrm{OH}$, es decir que:

$$
I N T O H=\int_{0}^{t}[O H] d t
$$

La escala MIR [20, 22-24] se define como el cambio máximo de concentración de ozono formado, una vez que se adiciona o modifica la concentración de un COV determinado y se consume una cantidad determinada de NO. Se calcula por medio de la relación:

$$
M I R_{C O V}=\lim (\Delta[C O V] \rightarrow 0)\left\{\frac{R(\Delta[C O V])-R(0)}{\Delta[C O V]}\right\}=\frac{d R}{d[C O V]}
$$

donde MIR es la máxima reactividad de los COV bajo las condiciones propias del escenario, $\mathrm{R}(0)$ es la máxima concentración de $\left(\left[\mathrm{O}_{3}\right]-\mathrm{NO}\right)$ calculada en un caso base (inicial), $\mathrm{R}\left(\Delta[\mathrm{COV}]\right.$ es la máxima concentración de $\left(\left[\mathrm{O}_{3}\right]-\mathrm{NO}\right)$ calculado en un escenario de simulación con la adición de un COV determinado al caso base, y $\Delta[\mathrm{COV}]$ es la concentración (en partes por millón de carbón) de los COV que están presentes al final de la simulación, si el compuesto orgánico adicionado no sufre ninguna reacción quimica, (ver Tabla 1.6).

La Tabla 1.6 muestra el potencial de cada molécula para formar de ozono. Así, el metano es compuesto que casi no forma ozono. Las demás parafinas presentan un potencial para formar ozono que se incrementa conforme aumenta el número de átomos carbonos. Los compuestos aromáticos, presentan un potencial mayor para formar ozono, con respecto a las parafinas, a excepción de benceno. 
Tabla 1.6 Escalas de reactividad OH y MIR.

\begin{tabular}{|l|l|l|}
\hline \multicolumn{1}{|c|}{ COV } & Escala OH & Escala MIR \\
\hline Metano & 0.1 & 0.0 \\
Etano & 3.2 & 2.7 \\
Propano & 9.3 & 6.2 \\
n-Butano & 15.3 & 11.7 \\
IsoButano & 14.2 & 15.7 \\
n-Pentano & 19.4 & 12.1 \\
Neo-Pentano & - & - \\
Benceno & 5.7 & 5.3 \\
Tolueno & 23.4 & 34 \\
o-Xileno & 48.3 & 87 \\
p-Xileno & 49.7 & 89 \\
m-Xileno & 80.2 & 109 \\
1,3,5-Trimetilbenceno & 159 & 140 \\
1,2,3-Trimetilbenceno & 89 & 119 \\
1,2,4-Trimetilbenceno & 107 & 119 \\
\hline
\end{tabular}




\section{CAPITULO 2}

\section{ANTECEDENTES TEORICOS Y EXPERIMENTALES}

En este capitulo se presenta una revisión de lo que se ha realizado hasta ahora en el tratamiento teórico y experimental más relevante con respecto a las reacciones de oxidación de parafinas e hidrocarburos aromáticos.

Las reacciones de abstracción de átomos de hidrógeno por radicales libres son procesos importantes en muchos aspectos de la quimica orgánica y de polimeros, así como en procesos atmosféricos y de combustión, y han sido ampliamente estudiadas, tanto experimental como teóricamente.

\subsection{Reacciones atmosféricas de parafinas}

Las reacciones de las parafinas con el radical $\mathrm{OH} \bullet$ proceden a través de la abstracción de un átomo de hidrógeno, para formar el radical metilo (R•) y el agua, i. e;

$$
\mathrm{RH}+\mathrm{OH} \bullet \rightarrow \mathrm{R} \bullet+\mathrm{H}_{2} \mathrm{O}
$$

Por otra parte, se sabe que bajo condiciones atmosféricas, el radical $\mathrm{R} \bullet$ reacciona con el oxigeno molecular para formar radicales peroxilo $\left(\mathrm{RO}_{2} \bullet\right)$ :

$$
\mathrm{R} \bullet+\mathrm{O}_{2} \rightarrow \mathrm{RO}_{2} \bullet
$$

La Tabla 2.1 muestra las constantes de velocidad de algunas reacciones de parafinas con el radical $\mathrm{OH} \bullet$, asi como del radical $\mathrm{R} \bullet$ con el $\mathrm{O}_{2}$.

En atmósferas contaminadas, donde existe una alta concentración de $\mathrm{NO}_{\mathrm{x}}$, los radicales $\mathrm{RO}_{2} \bullet$ pueden reaccionar con óxido nitrico, formando el radical alcoxi (RO•) y el dióxido de nitrógeno:

$$
\mathrm{RO}_{2} \bullet+\mathrm{NO} \rightarrow \mathrm{RO} \bullet+\mathrm{NO}_{2}
$$

o también pueden formar compuestos alquilnitratos, 


$$
\mathrm{RO}_{2} \bullet+\mathrm{NO} \rightarrow \mathrm{RONO}_{2}
$$

Tabla 2.1 Constantes de velocidad a presión alta para la reacción $\mathrm{R}+\mathrm{OH} \bullet \mathrm{y} \mathrm{R} \bullet+\mathrm{O}_{2}$ a temperatura ambientea.

\begin{tabular}{|l|l|l|l|}
\hline \multicolumn{1}{|c|}{ Compuesto } & \multicolumn{1}{c|}{$\begin{array}{c}\mathbf{R}+\mathbf{O H} \\
10^{12} \times \mathrm{k}_{2.1} \\
\left(\mathrm{~cm}^{3} \text { moléculas }^{-1} \mathrm{~s}^{-1}\right)\end{array}$} & & \multicolumn{1}{c|}{$\begin{array}{c}\mathbf{R} \cdot+\mathbf{O}_{2} \\
10^{12} \times \mathrm{k}_{2.2} \\
\left(\mathrm{~cm}^{3} \text { moléculas }^{-1} \mathrm{~s}^{-1}\right)\end{array}$} \\
\hline & & Primario & \\
\hline Metano & 0.00618 & Metil & 1.8 \\
Etano & 0.254 & Etil & 7.8 \\
Propano & 1.12 & 1-Propil & 8.0 \\
n-Butano & 2.44 & 1-Butil & 7.5 \\
iso-Butano & 2.19 & 2-Metil-1-Propil & 2.9 \\
\hline & & Secundario & \\
\hline & & 2-Propil & 11.0 \\
& & 2-Butil & 16.6 \\
\hline & & Terciario & \\
\hline & & 2-Metil-2-Propil & 23.4 \\
\hline
\end{tabular}

a R. Atkinson. J. Phys. Chem. Ref. Data. 26, 215, (1997).

Mientras que en atmósferas limpias, donde la concentración de $\mathrm{NO}_{\mathrm{x}}$ es mínima o no existe, el radical $\mathrm{RO}_{2} \bullet$ pueden reaccionar con otro $\mathrm{RO}_{2} \bullet$ a través de auto-reacciones, para formar:

$$
\mathrm{RO}_{2} \bullet+\mathrm{RO}_{2} \bullet \leftrightarrow \mathrm{RO}_{4} \mathrm{R}
$$

Las reacciones (2.3) y (2.4) son las responsables de alterar la relación $\mathrm{NO}_{2} / \mathrm{NO}$, aunque normalmente se acepta que las reacciones (2.1) y (2.2) son las que determinan el potencial de las parafinas para formar ozono, debido a la alta velocidad de reacción que presentan, del orden de $1 \times 10^{12} \mathrm{~cm}^{3} \mathrm{~mol}^{-1} \mathrm{~s}^{-1}[25 \cdot 26]$.

$\mathrm{El} \mathrm{RO}_{4} \mathrm{R}$ formado en la reacción (2.5), es un aducto o intermediario propuesto por algunos autores [27-28], para explicar algunos resultados experimentales, sugiriendo la existencia de un puente con cuatro oxigenos, cuya estructura podría ser lineal. T. Minato et al. [29] a través de cálculos por orbitales moleculares, proponen que el sistema con el puente de cuatro oxigenos podria ser un estado de transición. 


\subsection{Reacciones atmosféricas de compuestos aromáticos}

Los hidrocarburos aromáticos reaccionan con el radical $\mathrm{OH} \bullet$ por dos posibles caminos [1,25]: a) la reacción de abstracción de un átomo de hidrógeno del grupo metilo por el radical $\mathrm{OH} \bullet$, produciendo el radical bencilo más agua y b) la reacción de adición del radical $\mathrm{OH} \bullet$ al anillo aromático, produciendo el radical metil-hidroxi-ciclohexadienil (MHCHD):

$$
\begin{aligned}
& \mathrm{ArH}+\mathrm{OH} \bullet \rightarrow \mathrm{Ar} \bullet+\mathrm{H}_{2} \mathrm{O} \\
& \mathrm{ArH}+\mathrm{OH} \bullet \leftrightarrow \operatorname{ArH}(\mathrm{OH}) \bullet
\end{aligned}
$$

Los radicales Ar• y $\mathrm{ArH}(\mathrm{OH}) \bullet$, son muy estables, el primero (radical bencilo) se estabiliza por resonancia de los dobles enlaces en el anillo aromático, mientras que el segundo (radical MHCHD) se estabiliza a través de la resonancia del electrón desapareado dentro del anillo aromático (Figura 2.1).
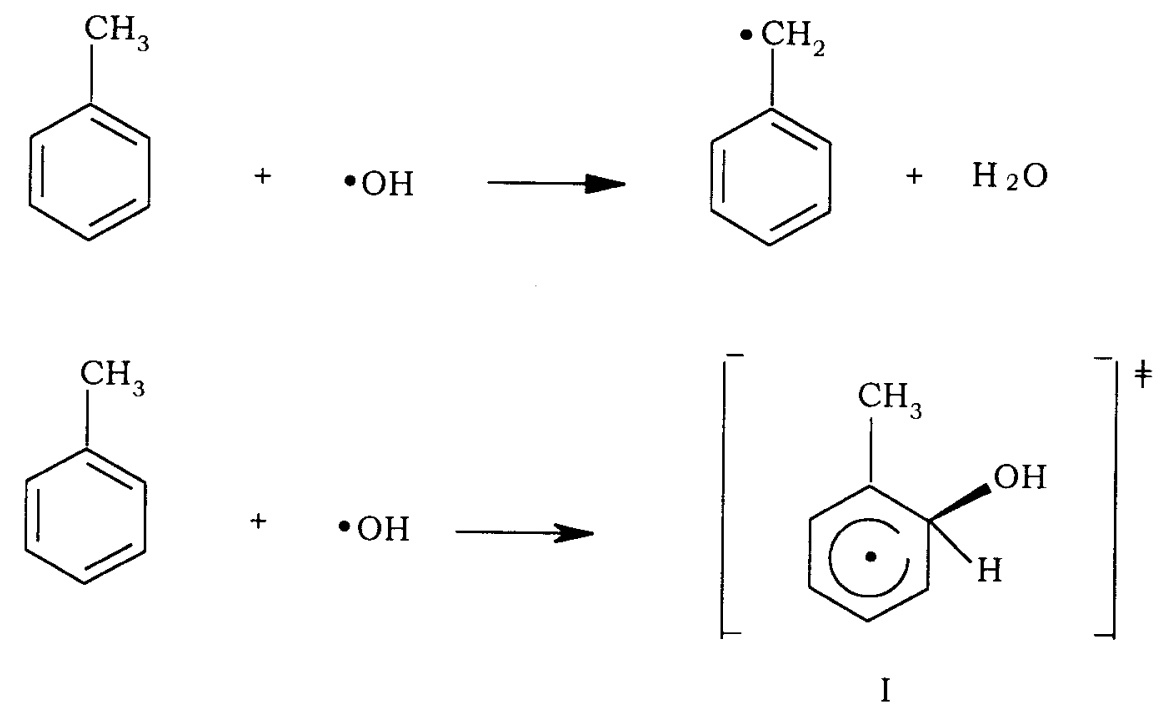

Figura 2.1 Reacciones de abstracción y de adición del tolueno con el radical OH• [25]. 
En la reacción de tolueno con el radical $\mathrm{OH} \bullet$ por abstracción de hidrógeno, después de formarse el radical bencilo se obtienen productos de reacción que conservan la estructura del anillo aromático, (Figura 2.2).

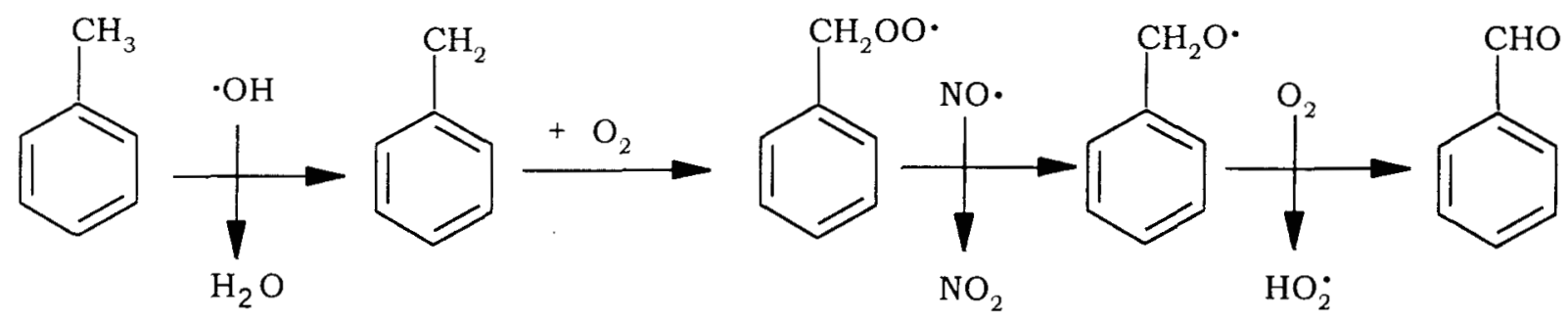

Figura 2.2 Productos de reacción que conservan la estructura del anillo aromático [25].

En la reacción de adición del radical $\mathrm{OH} \bullet$ al anillo aromático, se obtienen diferentes aductos (Figura 2.3) y productos de reacción derivados del rompimiento del anillo aromático (Figura 2.4). 


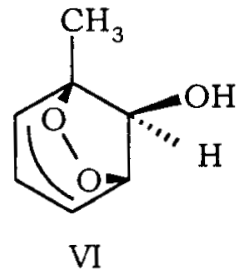

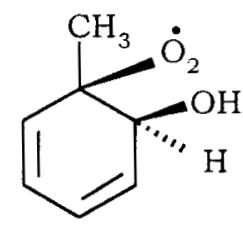

III

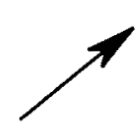<smiles>CC1=CC=C[C@H](O)[C@H]1O</smiles>

IV<smiles>CC1=CC([O-])C=C[C@H]1O</smiles><smiles>CC12CC[C@@H](C[C@@H]1O)O2</smiles>

VII<smiles>CC1COCCC1O</smiles><smiles>CC12CC3CC1OOC2(C)C3O</smiles>

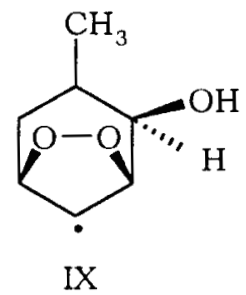

Figura 2.3 Aductos y biciclos de las reacciones de oxidación que sufre el tolueno [1]. 

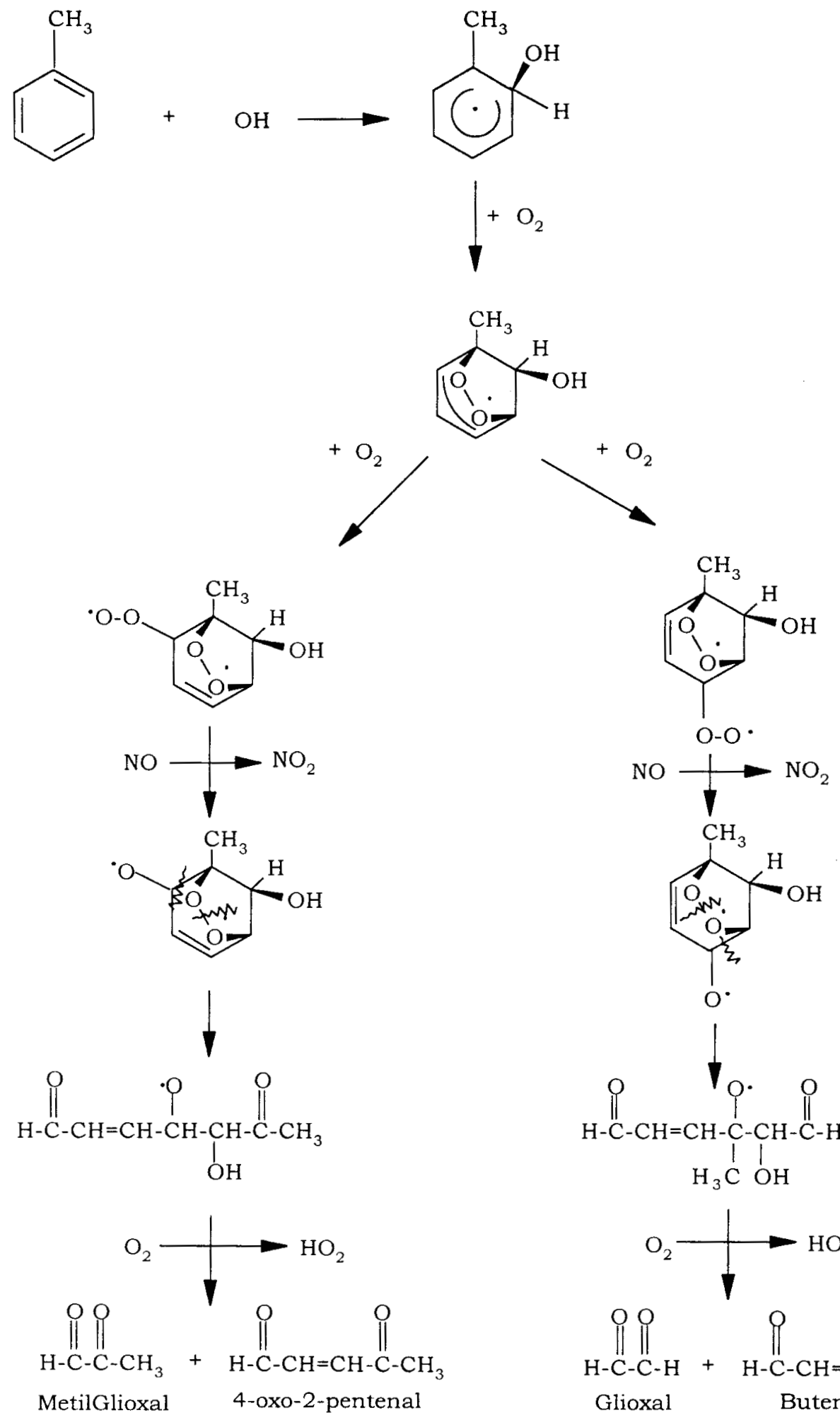

Figura 2.4 Productos de reacción después del rompimiento del anillo aromático [1]. 


\subsection{Estudios teóricos para reacciones de parafinas}

Ya en 1967 Kaufman et al. [30] realizaron cálculos de orbitales moleculares para la reacción,

$$
\mathrm{CH}_{4}+\mathrm{H} \bullet \rightarrow \cdot \mathrm{CH}_{3}+\mathrm{H}_{2}
$$

por medio del método de Hückel extendido. Morokuma y Davis [ ${ }^{31]}$ estudiaron la superficie de energia potencial para la misma reacción y también para el intercambio de hidrógeno, por medio de cálculos SCF con interacción de configuraciones (CI). Señalaron que la geometría calculada a nivel SCF es aproximadamente correcta pero que la introducción de los efectos de correlación es indispensable para la determinación de las energias. Los resultados obtenidos para las barreras de activación permitieron hacer predicciones cualitativas en cuanto a los caminos de reacción a diferentes temperaturas: así, a temperaturas bajas, sólo ocurre la reacción de abstracción, mientras que a altas temperaturas, también ocurre la de intercambio, aunque predomina la reacción de abstracción.

Para la reacción de metano con diferentes radicales $(\mathrm{R} \bullet)$,

$$
\mathrm{CH}_{4}+\mathrm{R} \bullet \rightarrow \mathrm{RH}+\bullet \mathrm{CH}_{3}
$$

existen cálculos más recientes, como el de Sana et al. [32], que optimizaron la geometria del estado de transición con el método UHF/6-31G, corrigiendo las energias con el método CIPSI-INO, (Tabla 2.2). En este trabajo, R• es, alguno de los siguientes substituyentes: $\mathrm{H} \bullet, \cdot \mathrm{CH}_{3}, \bullet \mathrm{NH}_{2}, \mathrm{OH} \bullet, \bullet F$. Los cálculos se realizaron con métodos ab-initio SCF para optimizar la geometría y con interacción de configuraciones para el cálculo de las energias. Los puntos más importantes de la curva de potencial a lo largo de la coordenada de reacción fueron ajustados con funciones cuadráticas y además se realizó un análisis vibracional. Se estimó el efecto túnel del átomo de hidrógeno a través de la barrera de reacción usando la aproximación de Wigner [33]. En este trabajo se encontró que el estado de transición presenta un ángulo de enlace $\mathrm{C}---\mathrm{H}---\mathrm{X}$, de $180^{\circ}$, cuando $\mathrm{X}$ es $\mathrm{H}, \mathrm{C}, \mathrm{y} \mathrm{F}$, mientras que cuando es $\mathrm{N}, \mathrm{y} \mathrm{O}$, el ángulo ya no es lineal $\mathrm{y}$ presenta una desviación de 6 y $7^{\circ}$, respectivamente. Además, en dicho trabajo, las frecuencias de vibración están sobreestimadas en un $10 \%$ al compararlas con las 
frecuencias experimentales. Para corregir esa sobreestimación realizaron un ajuste por regresión lineal, entre las frecuencias calculadas y las experimentales, encontrándose una relaciónde $v_{\exp }=0.90887 v_{\text {calc }}+37.36 \mathrm{~cm}^{-1}$, con un coeficiente de correlación de 0.991 .

Sana et al. [32] observaron que, en todos sus cálculos del estado de transición, se presenta una frecuencia imaginaria (negativa) de aproximadamente $1800 \mathrm{~cm}^{-1}$ que indica la localización de un estado de transición real. Las energias de activación tanto a nivel CI como con correcciones por el efecto túnel están sobreestimadas con respecto a las experimentales, alcanzándose desviaciones de más del 100\%. Los autores atribuyen estas desviaciones a un comportamiento no Arrhenius de la reacción estudiada.

Lluch et al. [34] compararon las reacciones de abstracción de un átomo de hidrógeno del metano, por diferentes radicales, $\mathrm{H} \bullet, \mathrm{OH} \bullet, \mathrm{Cl} \bullet \mathrm{y} \bullet \mathrm{CH}_{3}$, en un trabajo cuyo objetivo principal fue evaluar la capacidad del método semiempírico AM1 corregido con una versión modificada de la teoria de Marcus, para calcular barreras de activación. Los resultados son razonablemerıte adecuados comparándolos con resultados experimentales.

Para la reacción,

$$
\mathrm{CH}_{4}+\mathrm{OH} \bullet \rightarrow \bullet \mathrm{CH}_{3}+\mathrm{H}_{2} \mathrm{O}
$$

Dorigo y Houk [35] calcularon la estructura electrónica del estado de transición con el método HF/3-21G, y la compararon con la estructura obtenida por Whiteside et al. [36] con MP2/6-31 $\mathrm{G}^{\star \star}$, (Tabla 2.2). El objetivo de Dorigo y Houk fue estudiar el efecto causado en las energias de activación al distorsionar ligeramente la estructura del estado de transición, aumentando la distancia C---O, o cerrando el ángulo C---H---O, para el desarrollo de un potencial para el estudio de la transferencia de átomos de hidrógeno intermolecular en sistemas grandes, tal como en el caso de la formación de tetrahidrofuranos a partir de alcoholes.

Truong y Truhlar [ 37 estudiaron la misma reacción de metano con $\mathrm{OH}$ con los modelos UHF (Unrestricted Hartree-Fock) y MP2 (Møller-Plesset de segundo orden) y con diferentes bases, optimizando las estructuras y calculando, energías, y frecuencias vibracionales de los reactivos, estados de transición y productos. Para mejorar el cálculo de energias utilizaron un factor de escala, de acuerdo a la ecuación: 


$$
E_{\text {corregida }}=E_{U H F}+\frac{E_{U P M 2}-E_{U H F}}{F}
$$

que incluyen las correcciones Møller-Plesset provenientes de excitaciones de los electrones del carozo, utilizadas para estimar la barrera de activación (Tabla 2.2) y la constante de velocidad de dicha reacción. Cabe señalar que, los cálculos a nivel MP2 anteriores a 1995, en general reportan energias menores, ya que incluyen las excitaciones de los electrones internos.

Tabla 2.2 Parámetros geométricos de los reactivos, productos y estado de transición calculados con diferentes modelos reportados en la literatura.

\begin{tabular}{|c|c|c|c|c|c|c|c|c|c|}
\hline Mètodo & $\mathrm{CH}_{4}(\mathrm{~T} d)$ & $\mathbf{O H}\left(\mathbf{C}_{\infty \nu}\right)$ & \multicolumn{2}{|c|}{$\mathrm{H}_{2} \mathrm{O}\left(\mathrm{C}_{2 v}\right)$} & $\mathrm{CH}_{3}\left(\mathrm{D}_{3 h}\right)$ & \multicolumn{4}{|c|}{$\mathrm{H}_{3} \mathrm{C}---\mathrm{H}---\mathrm{OH}\left(\mathrm{C}_{s}\right)$} \\
\hline & $\mathrm{C}-\mathrm{H}$ & $\mathrm{O}-\mathrm{H}$ & $\mathrm{O}-\mathrm{H}$ & $\mathrm{HOH}$ & $\mathrm{C}-\mathrm{H}$ & $\mathrm{C}--\mathrm{H}_{\mathrm{a}}$ & $\mathrm{O}--\mathrm{H}_{\mathrm{a}}$ & $\mathrm{C}--\mathrm{H}--\mathrm{O}$ & $\mathrm{HOH}$ \\
\hline${ }^{\circ} \mathrm{UHF} / 3-21 \mathrm{G}$ & 1.0829 & 0.9858 & 0.9667 & 107.6 & 1.0717 & 1.3640 & 1.1714 & 171.49 & 102.25 \\
\hline${ }^{+} \mathrm{UHF} / 6-31 \mathrm{G}$ & 1.0882 & 0.9670 & 0.9480 & 111.50 & 1.0720 & 1.3340 & 1.1920 & 186.50 & 106.50 \\
\hline${ }^{0} \mathrm{HF} / 6-31 \mathrm{G}^{\star *}$ & 1.0816 & 0.9549 & 0.9431 & 105.97 & 1.0728 & 1.2986 & 1.2052 & 176.10 & 99.91 \\
\hline${ }^{0} \mathrm{HF} / 6-311 \mathrm{G}^{* *}$ & 1.0842 & 0.9514 & 0.9410 & 105.50 & 1.0735 & 1.2960 & 1.2065 & 173.68 & 99.30 \\
\hline $\begin{array}{l}{ }^{6} \mathrm{HF} / \\
6-311 \mathrm{G}(2 \mathrm{~d}, \mathrm{p})\end{array}$ & 1.0877 & 0.9523 & 0.9418 & 105.56 & 1.0722 & 1.2950 & 1.2081 & 173.48 & 99.30 \\
\hline $\begin{array}{l}{ }^{\circ} \mathrm{HF} / \\
6-311(3 \mathrm{~d}, 2 \mathrm{p})\end{array}$ & 1.0818 & 0.9496 & 0.9396 & 105.60 & 1.0706 & 1.2899 & 1.2120 & 175.05 & 99.38 \\
\hline${ }^{\circ} \mathrm{MP} 2 / 6-31 \mathrm{G}^{\star *}$ & 1.0843 & 0.9711 & 0.9608 & 103.87 & 1.0735 & 1.2034 & 1.2817 & 170.56 & 98.43 \\
\hline $\begin{array}{l}{ }^{\otimes} \mathrm{UMP} 2 / \\
6-31 \mathrm{G}^{\star *}\end{array}$ & & & & & & 1.2040 & 1.2810 & 169.18 & 98.52 \\
\hline $\begin{array}{l}\text { MP2/ } \\
6-311 \mathrm{G}^{\star \star}\end{array}$ & 1.0894 & 0.9658 & 0.9572 & 102.51 & 1.0786 & 1.1986 & 1.2938 & 168.81 & 96.73 \\
\hline $\begin{array}{l}{ }^{\mathrm{MP} 2 /} \\
6-311 \mathrm{G}(2 \mathrm{~d}, \mathrm{p})\end{array}$ & 1.0877 & 0.9687 & 0.9597 & 103.65 & 1.0765 & 1.1976 & 1.2977 & 168.10 & 97.28 \\
\hline $\begin{array}{l}\text { "MP2/ } \\
6-311(2 d, 2 p)\end{array}$ & 1.0828 & 0.9646 & 0.9563 & 103.38 & 1.0720 & 1.1906 & 1.3037 & 170.82 & 97.14 \\
\hline $\begin{array}{l}{ }^{9} \text { MP-SAC2 } / \\
6-311(2 \mathrm{~d}, \mathrm{p}) \\
\end{array}$ & 1.0885 & 0.9712 & 0.9626 & 103.34 & 1.0772 & 1.1834 & 1.3239 & 166.94 & 96.85 \\
\hline
\end{tabular}

*M. G. Sana, G. Leroy, and I. L. Villaveces. Theoret. Chim. Acta (Berl.) 65, 109, (1984).

-A. E. Dorigo, and K. N. Houk. J. Org. Chem. 53, 1650, (1988).

0 T. N. Truong, and D. G. Truhlar. J. Chem. Phys. 93, 1761, (1990).

$\otimes$ C. Gonzalez, J. J. W. McDouall, and H. B. Schlegel. J. Phys. Chem. 94, 7467, (1990).

Gonzalez et al. [38], estudiaron la reacción de abstracción del metano, abstrayendo un átomo de hidrógeno con $\bullet \mathrm{H}, \bullet \mathrm{O}, \mathrm{y} \mathrm{OH} \bullet$, con el objeto de estimar las constantes de velocidad de reacción, a través del formalismo del estado de transición no variacional, incluyendo correciones de Wigner, para el efecto túnel. Los resultados de este trabajo, se reportan también en la Tabla 2.2. 
Melissas y Truhlar [39] probaron su teoria variacional interpolada del estado de transición con la reacción del metano con el radical $\mathrm{OH} \bullet$. En este trabajo, los cálculos para los reactivos, productos y el estado de transición, los realizaron con el método MP2 y una base triple- $Z$ no estándar escalada por un factor adecuado a cada enlace. Melissas y Truhlar obtienen dos posibles estructuras para el estado de transición, una con el hidrógeno del $\mathrm{OH}$ en posición eclipsada y otra en posición alternada, con respecto a los hidrógenos del grupo metilo. En trabajos previos, realizados por Gordon y Truhlar [40], y Gonzalez et al. [38], se habia encontrado que la estructura más estable, a nivel UHF, era la alternada.

Con estos resultados, se originó la controversia acerca de cual era la estructura más estable, a nivel UHF o a nivel MP2. Melissas y Truhlar [44], resolvieron este problema, calculando la barrera de rotación entre ambas estructuras, encontrando que esta barrera es tan pequeña, de aproximadamente $0.02 \mathrm{kcal} / \mathrm{mol}$, que el estado de transición es prácticamente un rotor interno libre. No obstante, cabe señalar que la estructura eclipsada es la más estable a nivel MP2, si se utilizan bases cuidadosamente ajustadas. En el trabajo de Melissas y Truhlar [39], utilizan bases ajustadas de orbitales a las que llaman adj-cc-pVTZ (adjusted correlation consistent polarized Valence Triple Z), que contienen, para el átomo de $\mathrm{C}$ y $\mathrm{O}$, un conjunto de [10s $5 \mathrm{p} 2 \mathrm{~d}$ 1f] orbitales gaussianas primitivas contraidas a [4s $3 \mathrm{p} 2 \mathrm{~d}$ 1f], y para el átomo de hidrógeno orbitales gaussianas [5s $2 \mathrm{p} 1 \mathrm{~d}]$ contraidas a $\left[\begin{array}{lll}3 \mathrm{~s} & 2 \mathrm{p} & 1 \mathrm{~d}\end{array}\right]$. Según estos autores, este conjunto de orbitales gaussianas ajusta de manera óptima y balanceada la correlación electrónica, tanto en la formación como en la ruptura de enlaces.

Para esta misma reacción (metano con $\mathrm{OH}$ ), se han reportado, en la literatura, constantes de velocidad y energias de activación calculados por el método semiclásico CVT-SCT (Canonical Variational Transition State theory with Small Curvature Tunneling corrections), desarrollado por Gonzalez-Lafont et al. [41]. Los resultados de este trabajo muestran una buena correlación con los datos experimentales de la constante de velocidad de reacción en función de la temperatura. En este trabajo, de manera particular, se establece que el efecto túnel es muy importante entre 300 y $500 \mathrm{~K}$ y que más de la mitad de los eventos que culminan en la formación de productos ocurren por efecto túnel.

Para la reacción,

$$
\mathrm{C}_{2} \mathrm{H}_{6}+\mathrm{OH} \bullet \rightarrow \bullet \mathrm{C}_{2} \mathrm{H}_{5}+\mathrm{H}_{2} \mathrm{O}
$$


Melissas y Truhlar [42] realizaron cálculos de estructura electrónica usando la teoria de perturbaciones Møller-Plesset de segundo orden y bases ajustadas de orbitales adj-cc-pVTZ. En este estudio, la energia de correlación se escala con MP2-SAC (scaling all correlation energy), para mejorar el cálculo de la barrera de reacción. Estos autores obtienen que, la altura de la barrera directa e inversa es de 4 y $21.6 \mathrm{kcal} / \mathrm{mol}$, respectivamente, con una diferencia de $17.30 \mathrm{kcal} / \mathrm{mol}$ que está de acuerdo con resultados experimentales. Aplicando su teoria variacional interpolada del estado de transición y utilizando la información de las alturas de las barreras, determinan la constante de velocidad, para esta reacción, en un rango de temperaturas de 200 a 3000 $\mathrm{K}$. Los resultados teóricos obtenidos por Melissas y Truhlar [17] concuerdan con los resultados experimentales en el mismo rango de temperatura, reportados en la literatura.

Sekusak et al. [43] estudiaron la reacción de abstracción de un átomo de hidrógeno de etano y etano halogenado con el radical $\mathrm{OH} \bullet$, para calcular las velocidades de reacción en un rango de temperatura de 200 a $1000 \mathrm{~K}$, empleando el método CVT-SCT. Las estructuras de minima energia fueron calculadas a un nivel de teoria $\mathrm{MP} 2$ (full) $/ 6-31 \mathrm{G}^{* *}$, haciendo correcciones para la altura de la barrera con la metodologia G2(MP2), implementada en el paquete de programas Gaussian94. Observaron que, en las reacciones de abstracción de hidrógeno el efecto túnel juega un papel muy importante, ya que incrementa la velocidad de reacción por un factor de 5 a $200 \mathrm{~K}$, y por un factor de 2 a $320 \mathrm{~K}$. Cabe señalar que este rango de temperatura, es donde se llevan a cabo la mayoria de las reacciones de oxidación de los hidrocarburos en la tropósfera.

Para la reacción,

$$
\mathrm{C}_{3} \mathrm{H}_{8}+\mathrm{OH} \bullet \rightarrow \cdot \mathrm{C}_{3} \mathrm{H}_{7}+\mathrm{H}_{2} \mathrm{O}
$$

es necesario diferenciar la abstracción un átomo de hidrógeno primario de la de un átomo de hidrógeno secundario. Hu et al. ${ }^{[44]}$, modelaron el proceso de combustión del propano con el radical $\mathrm{OH} \bullet$, usando la teoria variacional del estado de transición, en conjunto con métodos de dinámica molecular y cálculos de estructura electrónica con el método MP2/aug-cc-pVDZ (Dunning's augmented correlation-consistent polarized Valence Double Z). 
Para esta reacción, se encuentran tres estructuras correspondientes al estado de transición. La abstracción de un átomo de hidrógeno primario, presenta dos estructuras, una alternada y otra eclipsada, siendo esta última la más estable, mientras que la abstracción de un átomo de hidrógeno secundario presenta una estructura eclipsada. La reacción de abstracción de un átomo secundario, presenta la barrera más baja. Las alturas de las barreras obtenidas se introdujeron en métodos de dinámica molecular, para determinar las velocidades de reacción. Los resultados teóricos son comparables con resultados experimentales.

Jungkamp et al. [45] estudiaron el mecanismo de oxidación atmosférica del nbutano que se inicia con la reacción:

$$
\mathrm{C}_{4} \mathrm{H}_{10}+\mathrm{OH} \bullet \rightarrow \bullet \mathrm{C}_{4} \mathrm{H}_{9}+\mathrm{H}_{2} \mathrm{O}
$$

Estos autores utilizaron la teoría de funcionales de la densidad y encontraron dos rutas principales de reacción: i) la formación del radical 1-butoxi y ii) la formación del radical 2 -butoxi, los cuales dan origen a productos que se miden experimentalmente: el 4hidro-1-butanal y 4-hidroperoxy-1-butanal.

Más recientemente, Aliagas y Gronert [46] estudiaron con métodos ab-initio, la reacción de metano, etano, propano (radical secundario), e isobutano (radical terciario) con el radical $\mathrm{OH} \bullet$, utilizando los modelos G2 y G2(MP2) implementados en el programa Gaussian94. Los dos métodos generan resultados similares, aunque lo más interesante en ambos métodos es que subestiman tanto las entalpias como las energias de activación por más de $10 \mathrm{kcal} / \mathrm{mol}$.

\subsection{Estudios teóricos de radicales peroxilo $\mathrm{RO}_{2} \bullet$ de las parafinas}

La estructura electrónica y otras propiedades moleculares de los radicales $\mathrm{RO}_{2} \bullet$ de hidrocarburos, se han estudiado mediante cálculos de orbitales moleculares que han permitido establecer propiedades electrónicas del estado basal y de los primeros estados excitados. Desde el punto de vista de los orbitales moleculares que participan en la formación de los radicales peroxilo, se establece que éstos se forman a través de la interacción de un radical orgánico con su electrón desapareado que se encuentra en un orbital $\sigma$ enlazante y el orbital $\pi$ con dos electrones desapareados de una molécula de oxigeno en su estado basal, lo que da lugar a la formación del radical peroxilo en el que el 
electrón desapareado finalmente se encuentra en un orbital del tipo $\pi$ del oxigeno terminal.

S. Benson [47, con sus reglas de aditividad de grupos termodinamicos ha estimado la estabilidad y la cinética química de radicales alquil peroxilo. Lightfoot et al. [48] hicieron una revisión detallada sobre estudios teóricos y experimentales de los radicales peroxilo.

Los radicales alquil peroxilo más estudiados a la fecha por los métodos de la quimica cuántica son el metil, etil, propil, alil, butil, e isobutil peroxilos. Boyd et al. [49] estudiaron las propiedades de alguno de esos peroxilos, con especial énfasis en la importancia de la correlación electrónica. Jafri y Phillips [50] utilizaron cálculos ab-initio para encontrar los principales canales de disociación del radical metil peroxilo. Wetmore y Boyd [51] más recientemente determinaron con teoria de funcionales de la densidad y diferentes funciones base, las constantes de acoplamiento hiperfino de algunos radicales alquil peroxilo.

\subsection{Estudios teóricos para reacciones de compuestos aromáticos}

A la fecha, la reacción de abstracción de hidrógeno de la cadena lateral de los compuestos aromáticos por el radical $\mathrm{OH} \bullet$, no ha sido estudiada teóricamente. En este trabajo, presentamos los primeros resultados de esta reacción a nivel ab-initio donde obtenemos una energía de activación muy alta (Capitulo 4). Estos resultados están en desacuerdo con los pocos datos experimentales de energía de activación de $0.9 \mathrm{kcal} / \mathrm{mol}$, para esta reacción.

En 1991 Tanaka et al. [52], estudiaron la reacción de abstracción de un átomo de hidrógeno de antioxidantes fenólicos, en procesos de auto-oxidación en cadena, a través de cálculos ab-initio. En este estudio, se observó que los grupos electrodonadores o aquellos que pueden deslocalizar el electrón desapareado en el anillo aromático, incrementan la actividad antioxidante, mientras que los grupos electroatrayentes la disminuyen.

La reacción inicial más importante en la oxidación de los compuestos aromáticos, es la adición del radical $\mathrm{OH} \bullet$ al anillo aromático. Así, en 1995 Bartolotti y Edney [53] con teoria de funcionales de la densidad (DFT), estudiaron la oxidación atmosférica del tolueno, a través de esta reacción. La adición del radical $\mathrm{OH} \bullet$ al anillo aromático, en una 
primera etapa, forma el radical MHCHD. En este estudio se observó que el radical MHCHD presenta una gran estabilidad a través de la resonancia del electrón desapareado dentro del anillo aromático. El radical $\mathrm{MHCHD}$ puede reaccionar con $\mathrm{O}_{2}$, en las posiciones 1, 3 y 5 , con respecto al grupo metilo, para formar el radical peroxilo correspondiente. En este caso, la estructura que más se favorece es la adición del $\mathrm{O}_{2}$ en la posición 3. Los radicales peroxilo forman un biciclo a través de un puente de oxigeno, que finalmente dan origen a un radical tipo epóxido.

En 1996 Andino et al. [54] estudiaron el mecanismo de adición del radical OH• y del $\mathrm{O}_{2}$ a hidrocarburos alquil-bencénicos. En estos estudios la geometria de los reactivos, productos y estados de transición, se optimizaron inicialmente a nivel UHF/PM3. Posteriormente las energias se mejoraron con $\mathrm{B} 3 \mathrm{LYP} / 6-31 \mathrm{G}^{\star \star}$ (SP, single point). Al igual que Bartolotti y Edney [53], Andino et al. [54] establecen que la adición del $\mathrm{O}_{2}$ se favorece principalmente en la posición 3. En este trabajo no se menciona la posibilidad de formar estructuras tipo epóxido, como en el estudio de Bartolotti y Edney [53].

Más recientemente, Ghigo y Tonachinni $1998{ }^{[55]}$ estudiaron con métodos de DFT, tres canales de reacción del radical hidroxi-ciclo-hexa-dienil ( $\mathrm{HCHD)}$ con el $\mathrm{O}_{2}$. En el primer canal, el $\mathrm{O}_{2}$ abstrae al hidrógeno del carbono donde está enlazado el radical $\mathrm{OH} \bullet$ (hidrógeno geminal), para posteriormente formar fenol. En el segundo canal, el $\mathrm{O}_{2}$ se adiciona al radical HCHD para formar el radical peroxilo aromático (ArOO•). Y en el tercer canal, el $\mathrm{O}_{2}$ abstrae al átomo de hidrógeno del $\mathrm{OH}$ para formar un epóxido, el cual, al romperse el enlace $\mathrm{C}-\mathrm{C}$ del grupo epóxido genera un epóxido cíclico de ocho átomos, $\mathrm{u}$ oxepina, cuya formación no es muy favorecida.

\subsection{Estudios experimentales de compuestos aromáticos}

Se han reportado una gran cantidad de datos experimentales [56-63] para las reacciones de abstracción de hidrógeno por el radical $\mathrm{OH} \bullet$, asi como para las reacciones de adición del radical $\mathrm{OH} \bullet$ a los hidrocarburos tanto en hidrocarburos saturados como en no saturados. Atkinson [57-59] ha medido experimentalmente la constante de velocidad de reacción de estos sistemas, ajustándola a la expresión empirica $\mathrm{k}=\mathrm{CT} \mathrm{T}^{\mathrm{n}} \exp ^{-\mathrm{D} / \mathrm{T}}$.

Respecto a los compuestos aromáticos se sabe que tienen una gran importancia en atmósferas urbanas altamente contaminadas. En particular, el tolueno representa más del $80 \%$ de los compuestos aromáticos que se emiten a la atmósfera. El proceso más importante para eliminar a los compuestos aromáticos de la atmósfera, es a través de su 
reacción con el radical $\mathrm{OH} \bullet$. A temperatura ambiente la reacción de adición predomina sobre la de abstracción, como lo indican dos evidencias experimentales. La primera evidencia es que la velocidad de reacción de $\mathrm{OH}$ • con el tolueno deuterado $\mathrm{C}_{6} \mathrm{D}_{5} \mathrm{CD}_{3}$, a temperatura ambiente es similar (dentro del error experimental) a la del tolueno sin deuterar $\mathrm{C}_{6} \mathrm{H}_{5} \mathrm{CH}_{3}$, mientras que a $423{ }^{\circ} \mathrm{K}$ la constante de velocidad del tolueno sin deuterar es 2 veces más alta [64-65]. Esta observación indica que a temperatura ambiente, en la ruta de reacción de abstracción no se presenta ruptura de enlace C-D o C-H. La segunda evidencia experimental, es que los productos que se obtienen a través del mecanismo de abstracción, constituyen menos del $20 \%$ del total de productos cuantificados, esto sin embargo, requiere de más investigación, ya que solo el $50 \%$ del tolueno inicial se cuantifica en dichos productos.

Knispel et al. [6] determinaron experimentalmente las constantes de velocidad de los hidrocarburos aromáticos con el radical $\mathrm{OH} \bullet$, bajo condiciones atmosféricas, a través de la formación de los aductos $\mathrm{OH}$-Aromático- $\mathrm{O}_{2}$ y $\mathrm{OH}$-Aromático- $\mathrm{NO}_{2}$. En este estudio observaron que la reacción con el $\mathrm{NO}_{2}$ es lenta, mientras que con el $\mathrm{O}_{2}$ es muy rápida, por lo que concluyen que en condiciones atmosféricas, los radicales aril peroxilos, Ar•, reaccionan casi exclusivamente con oxígeno molecular.

Klotz et al. ${ }^{[67]}$ proponen que la oxidación atmosférica del benceno se lleva a cabo a través del aducto $\mathrm{OH}-\mathrm{Benceno}-\mathrm{O}_{2}$, favoreciéndose en más del $50 \%$ la formación del fenol (estructura que no rompe el anillo aromático), mientras que el resto se distribuye entre la formación del radical hidroxi-ciclo-hexa-dienil peroxilo y la formación del oxido de benceno/oxepina, que posteriormente, llevan a productos de fragmentación del anillo aromático.

Más recientemente, en 1998, Klotz et al. ${ }^{[68]}$ presentaron nuevos resultados sobre la fotooxidación atmosférica del benceno y del tolueno, con base en el mecanismo propuesto en la referencia anterior, a través de los intermediarios óxido de benceno/oxepina para el benceno y 1,2-oxido de tolueno/2-metiloxepina para el tolueno. Lo interesante de este trabajo es que mientras el benceno favorece la formación de fenol en aproximadamente $(43.2 \pm 4.5) \%$, el tolueno únicamente forma el orto-cresol en aproximadamente $(2.7 \pm 2.2) \%$. Esto implica, que más bien favorece la formación de productos que rompen la estructura del anillo aromático. 


\section{CAPITULO 3}

\section{RESULTADOS Y DISCUSION REACCIONES DE OXIDACIÓN DE LAS PARAFINAS}

En este capítulo, reportamos los resultados del estudio de las estructuras y energias de los reactivos, productos y estados de transición de las principales reacciones de una serie de parafinas, metano, etano, propano, butano, iso-butano, y neo-pentano. Dichas reacciones son la abstracción de hidrógeno por el radical $\mathrm{OH} \bullet$, y la posterior adiciōn de oxígeno molecular para formar un radical peroxilo. Los cálculos fueron realizados a nivel UMP2/6-31G** y B3LYP/6-31G**.

\subsection{Reacción de abstracción de hidrógeno de parafinas por el radical $\mathrm{OH}$.}

La reacción de abstracción de hidrógeno por $\mathrm{OH} \bullet$ es la siguiente:

$$
\mathrm{RH}+\mathrm{OH} \bullet \rightarrow \mathrm{R} \bullet+\mathrm{H}_{2} \mathrm{O}
$$

Se reconoce que, en todas las reacciones de abstracción de hidrógeno de las parafinas por el radical $\mathrm{OH} \bullet$, la coordenada principal de reacción es esencialmente la distancia entre el oxigeno del radical $\mathrm{OH} \bullet \mathrm{y}$ un hidrógeno del carbono del grupo metilo $\mathrm{CH}_{3}$, o metileno $-\mathrm{CH}_{2-}$, a través del puente $\mathrm{C}---\mathrm{H}---\mathrm{O}$. En la Figura 3.1 se representa el estado de transición de la reacción para el caso del metano. Los estados de transición TS1 para la abstracción de un hidrógeno primario y secundario del butano y del hidrógeno terciario del iso-butano, se muestran en las Figuras 3.2a, 3.2b y 3.2c respectivamente. En estas figuras también se indican las principales características geométricas, distancias y ángulos de enlace. 


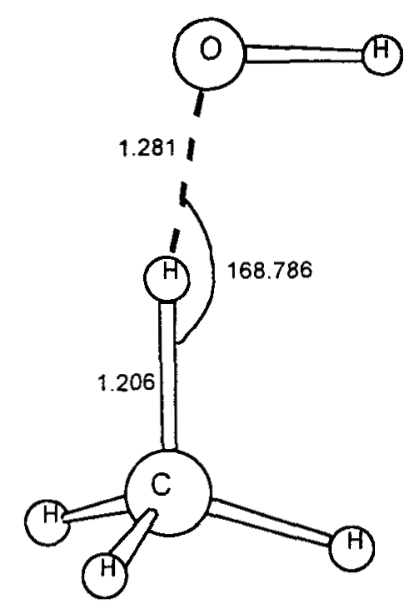

Figura 3.1 Optimización del estado de transición de la reacción de metano con el radical $\mathrm{OH} \bullet$ con $\mathrm{UMP} 2 / 6-31 \mathrm{G}^{\star *}$.

Encontramos que los valores de las distancias en las geometrias optimizadas del estado de transición TS1, para reacciones equivalentes (es decir, abstracción de un hidrógeno primario, secundario o terciario) de etano, propano, butano, iso-butano y neo-pentano son muy similares, con diferencias de tan solo $0.003 \AA$ en las longitudes de enlace y de $0.1^{\circ}$ en los ángulos de enlace. En general, todos los estados de transición TS1 calculados en este trabajo presentan las siguientes caracteristicas:

1) La distancia $\mathrm{C}-\mathrm{H}$ es más pequeña que la distancia $\mathrm{H}-\mathrm{O}$. Esto indica un estado de transición temprano, cuando el radical $\mathrm{OH} \bullet$ todavia está lejos. Este resultado concuerda con el postulado de Hammond, según el cual los estados de transición de reacciones exotérmicas deben ser más cercanos a los reactivos que a los productos. Además encontramos que el estado de transición es más temprano cuanto más substituido está el carbono. Así, para el caso de la reacción de abstracción de un hidrógeno primario del butano con el radical $\mathrm{OH} \bullet$, la distancia $\mathrm{C}-\mathrm{H}$ es de $1.188 \AA$ y la distancia $\mathrm{O}-\mathrm{H}$ es $1.330 \AA$, para la abstracción de un hidrógeno secundario la distancia C-H es $1.176 \AA$ y la distancia $\mathrm{O}-\mathrm{H}$ es $1.367 \AA$, mientras que para la abstracción de un hidrógeno terciario la distancia C-H es $1.166 \AA$ y la distancia O-H es $1.392 \AA$.

2) El ángulo de enlace $\mathrm{C}-\mathrm{H}-\mathrm{O}$ en el TS1 es cercano a $180^{\circ}$. El oxigeno del radical $\mathrm{OH} \bullet$, se inserta de forma perpendicular para abstraer al hidrógeno del hidrocarburo, mientras que el hidrógeno de éste radical queda en posición cis con respecto a la cadena 
de carbonos. Para la abstracción de un hidrógeno primario el ángulo $\mathrm{C}-\mathrm{H}-\mathrm{O}$ de $168.8^{\circ}$, para la abstracción de un hidrógeno secundario es de $168.9^{\circ}$ y para la abstracción de un hidrógeno terciario es de $175.1^{\circ}$. Este último es casi lineal debido al efecto estérico de los substituyentes.

3) La distancia total C---H---O para el TS1 de todos los sistemas estudiados es de alrededor de $2.5 \AA$, característico de un puente de hidrógeno, y concuerda con los resultados obtenidos por Dorigo et al. [35].

4) El TS1 para la abstracción de un hidrógeno primario presenta una sola frecuencia imaginaria, de aproximadamente $-1730 \mathrm{~cm}^{-1}$. En el caso de la abstracción de un hidrógeno secundario la frecuencia es un poco menor, alrededor de $-1421 \mathrm{~cm}^{-1}$, y para la abstracción de un hidrógeno terciario la frecuencia de vibración es aún menor, de $-1092 \mathrm{~cm}^{-1}$. La precencia de una sola frecuencia negativa indica que hemos localizado un estado de transición real. Las frecuencias de vibración menores en la abstracción de un hidrógeno secundario y terciario, indican una menor curvatura en la superficie de energia potencial, con respecto a la abstracción de un hidrógeno primario.

5) Un análisis de población de Mulliken utilizando la función de onda MP2 permite determinar las cargas y las densidades de espin sobre los átomos. En particular, para el TS1, para los tres átomos directamente involucrados en la coordenada de reacción, se obtienen las siguientes cargas: -0.35 para el carbono, -0.49 para el oxigeno, y de +0.25 para el hidrógeno. Las densidades de espin son $0.040,0.72$ y -0.09 respectivamente, para los mismos átomos, indicando una concentración importante del espin en el átomo de oxigeno. Para la abstracción de un hidrógeno secundadrio y terciario, la separación de cargas es menor y la densidad de espin sobre el oxigeno es menor también.

En la Figura 3.3 se muestran las geometrias optimizadas para las estructuras de los radicales alquilicos del butano y del iso-butano. Estas estructuras fueron investigadas cuidadosamente, para estar seguros de que correspondan a la estructura de minima energia. En particular, para el radical n-butil, la curva de energia potencial para la torsión del grupo metilo alrededor del enlace $\mathrm{C} 3-\mathrm{C} 4$ se obtuvo calculando la optimización completa de la geometria como una función de valores fijos del ángulo diedro, $\tau=\mathrm{HC} 4 \mathrm{C} 3 \mathrm{C} 2$. La estructura de minima energia es la que se presenta en la Figura 6.3. 


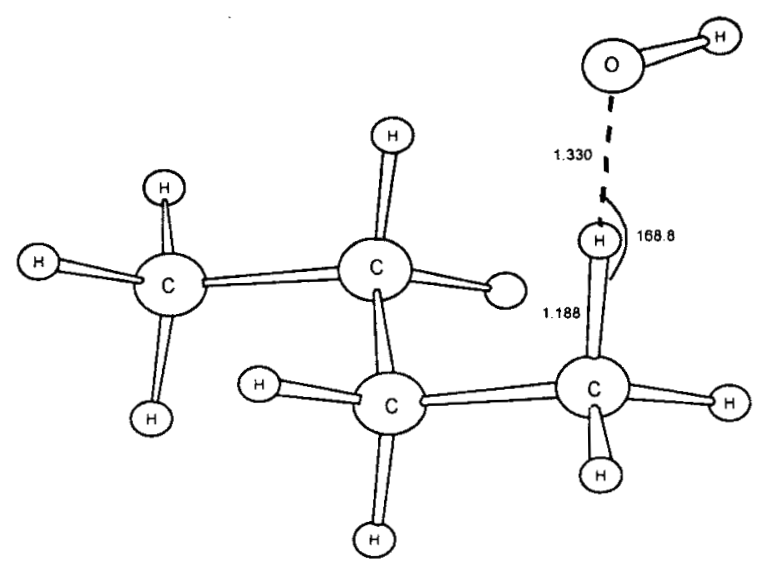

a)

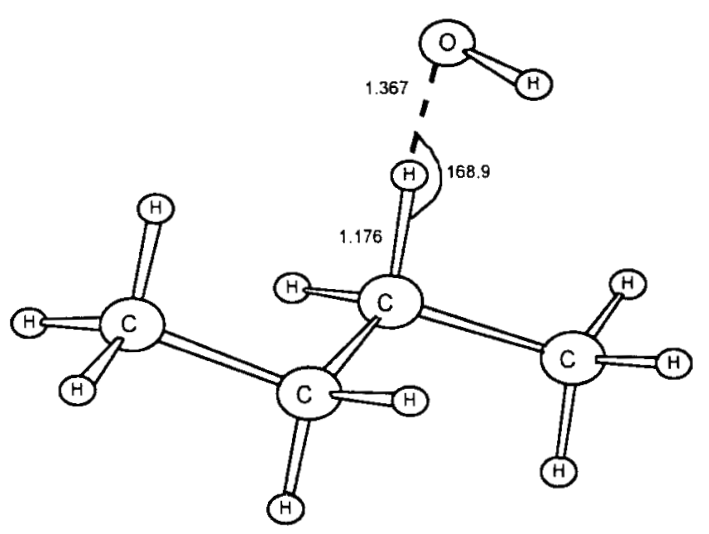

b)

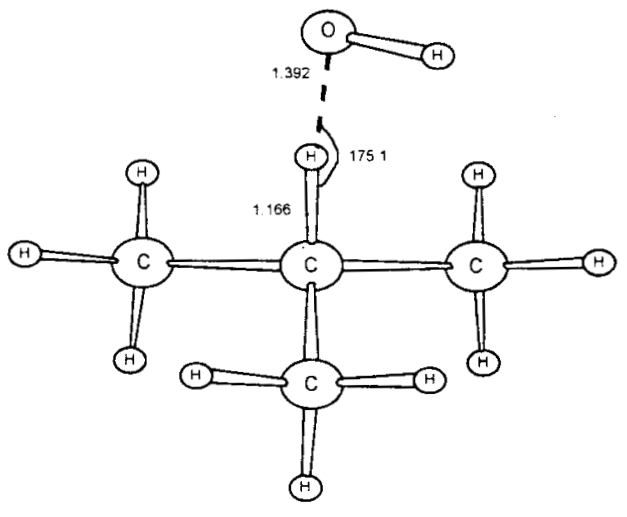

c)

Figura 3.2 Determinación del estado de transición de la reacción de abstracción de hidrógeno del butano y del iso-butano, por el radical $\mathrm{OH} \bullet$ a nivel UMP2/6-31G*: a) hidrógeno primario, b) hidrógeno secundario y c) hidrógeno terciario. 


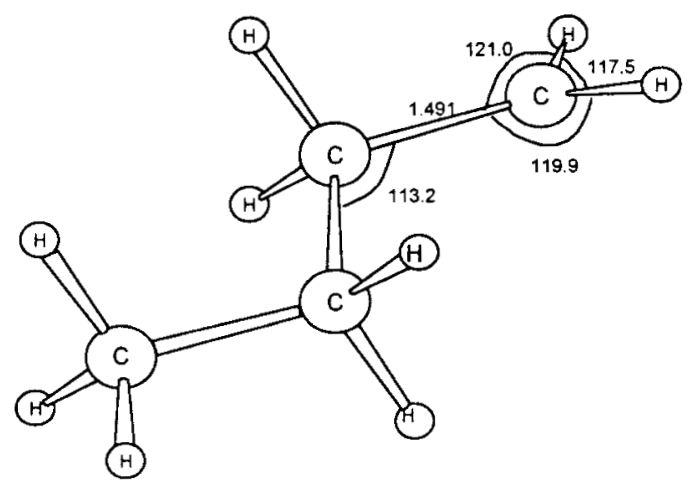

a)

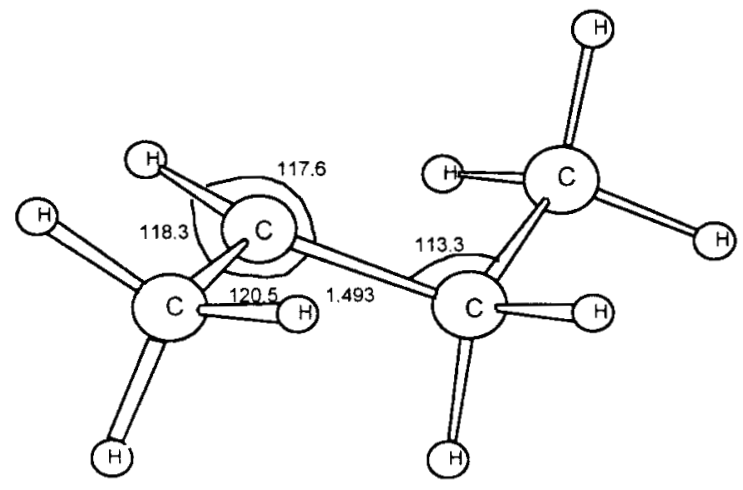

b)

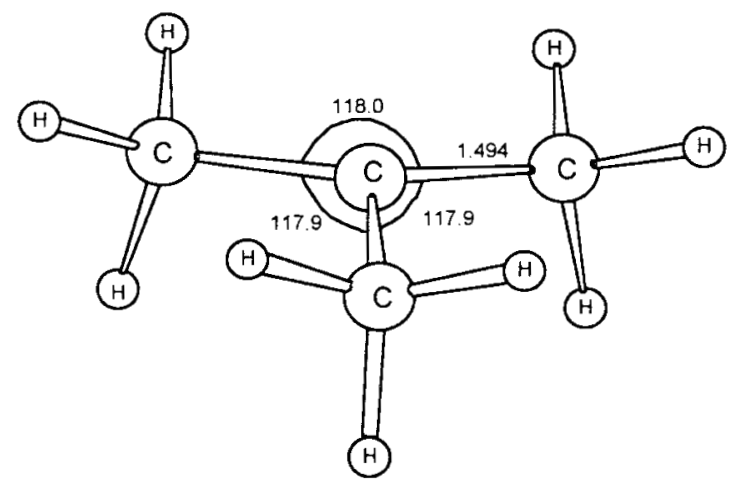

c)

Figura 3.3 Estructuras optimizadas de los radicales R• del butano y del iso-butano a nivel UMP2/6-31G**: a) Radical primario, b) Radical secundario y c) Radical terciario. 
La Tabla 3.1 muestra las energias PMP2/6-31G** que se obtienen después de proyectar la función de onda a nivel UMP2/6-31G**. En esta tabla, también se muestran los valores del cuadrado del momento angular intrinseco $\left(\mathrm{S}^{2}\right)$ antes y después de proyectar la componente del cuarteto. También se incluyen en esta tabla las frecuencias imaginarias que caracterizan los estados de transición. Además de las energias electrónicas totales, se presentan las correcciones del punto cero de las vibraciones y las correcciones térmicas, obtenidas al mismo nivel de cálculo. Se observa que el estado de transición para la reacción de abstracción de $\mathrm{H}$, TS1, presenta una contaminación de espin antes de la proyección de la función de onda, cuyo valor de $\mathrm{S}^{2}$ oscila entre $0.776 \mathrm{y}$ 0.779 dependiendo de la reacción, y una vez proyectada la función se obtiene siempre el valor esperado para un doblete, de 0.750 .

La Tabla 3.2 muestra los resultados de las energias (single point) con B3LYP/6$31 \mathrm{G}^{* \star}$ calculadas a las geometrias optimizadas con $\mathrm{MP2}$, asi como los correspondientes valores de $\mathrm{S}^{2}$ antes y después ae la proyección de la función de onda, para los reactivos, estados de transición y productos. Con este método la contaminación de espín es muy pequeña.

En la Tabla 3.3 se muestran las energias de reacción y energias de activación en $\mathrm{kcal} / \mathrm{mol}$, para la reacción de abstracción de hidrógeno de parafinas con el radical $\mathrm{OH} \bullet$, a nivel UMP2/6-31G**. Los resultados teóricos se comparan con dos series de datos experimentales. En esta tabla, las energias relativas han sido calculadas con respecto a los reactivos aislados, e incluyen la corrección térmica. Los resultados están agrupados de acuerdo al tipo de hidrógeno abstraído, a fin de resaltar las tendencias que muestran claramente la mayor reactividad de un hidrógeno terciario en comparación con las de hidrógenos secundarios y primarios.

Como se puede observar, en la Tabla 3.3, los valores absolutos de las energias de activación a nivel PMP2 difieren por un factor de más de 2 con respecto a los resultados experimentales reportados por Donahue et al. [991. Además de las aproximaciones involucradas en el método de cálculo, tales como las limitaciones en las funciones base y una descripción incompleta de la correlación electrónica, estas diferencias se pueden atribuir al hecho bien conocido de que estas reacciones ocurren, en buena medida por efecto túnel, de acuerdo a Melissas y Truhlar [39]. 
Tabla 3.1 Energias PMP2 en hartrees, correcciones de vibración en el punto cero (ZPE), correcciones térmicas (TE), valores de $\left\langle\mathrm{S}^{2}\right\rangle$ antes y después de la proyección de los reactivos, productos y estados de transicion.

\begin{tabular}{|c|c|c|c|c|c|c|}
\hline Sistema & $\begin{array}{l}\text { PMP2/ } \\
\text { 6-31G** }\end{array}$ & ZPE & TE & $\begin{array}{c}\left\langle\mathbf{S}^{2}\right\rangle \\
\text { Antes }\end{array}$ & $\begin{array}{c}\left\langle\mathbf{S}^{2}\right\rangle \\
\text { Después }\end{array}$ & $\begin{array}{c}\text { Frec. } \\
\text { Imag. } v i\end{array}$ \\
\hline $\mathrm{OH} \bullet$ & -75.533571 & 0.008753 & 0.011113 & 0.755 & 0.75 & \\
\hline $\mathrm{H}_{2} \mathrm{O}$ & -76.219788 & 0.021879 & 0.024714 & 0.0 & 0.0 & \\
\hline $\mathrm{O}_{2}$ & -149.955768 & 0.003211 & 0.005579 & 2.0 & 2.0 & \\
\hline \multicolumn{7}{|l|}{$\mathbf{R H}$} \\
\hline Metano & -40.364626 & 0.046544 & 0.049405 & 0.0 & 0.0 & \\
\hline Etano & -79.543400 & 0.077462 & 0.080885 & 0.0 & 0.0 & \\
\hline Propano & -118.725385 & 0.107094 & 0.111524 & 0.0 & 0.0 & \\
\hline Butano & -157.907453 & 0.136492 & 0.142158 & 0.0 & 0.0 & \\
\hline Iso-butano & -157.910074 & 0.136057 & 0.141617 & 0.0 & 0.0 & \\
\hline Neo-pentano & -197.096688 & 0.164663 & 0.171380 & 0.0 & 0.0 & \\
\hline \multicolumn{7}{|l|}{ TS1 } \\
\hline Metano & -115.882515 & 0.052506 & 0.057200 & 0.781 & 0.75 & -2061 \\
\hline Etano & -155.065167 & 0.083291 & 0.088907 & 0.779 & 0.75 & -1768 \\
\hline Propano $1^{\circ}$ & -194.248608 & 0.113254 & 0.119709 & 0.779 & 0.75 & -1730 \\
\hline Propano $2^{\circ}$ & -194.249914 & 0.112822 & 0.119726 & 0.777 & 0.75 & -1467 \\
\hline Butano $1^{\circ}$ & -233.430798 & 0.142208 & 0.150341 & 0.779 & 0.75 & -1760 \\
\hline Butano $2^{\circ}$ & -233.433217 & 0.142614 & 0.150542 & 0.777 & 0.75 & -1421 \\
\hline Iso-butano $1^{\circ}$ & -233.433382 & 0.142160 & 0.149864 & 0.779 & 0.75 & -1731 \\
\hline Iso-butano $3^{\circ}$ & -233.437124 & 0.141798 & 0.150043 & 0.776 & 0.75 & -1092 \\
\hline Neo-pentano $1^{\circ}$ & -272.620825 & 0.170859 & 0.179691 & 0.778 & 0.75 & -1690 \\
\hline \multicolumn{7}{|l|}{ Radical } \\
\hline Metano & -39.694621 & 0.030707 & 0.033866 & 0.761 & 0.75 & \\
\hline Etano & -78.877941 & 0.061740 & 0.065588 & 0.762 & 0.75 & \\
\hline Propano $1^{\circ}$ & -118.059025 & 0.090945 & 0.095202 & 0.763 & 0.75 & \\
\hline Propano $2^{\circ}$ & -118.063891 & 0.091668 & 0.096610 & 0.763 & 0.75 & \\
\hline Butano $1^{\circ}$ & -157.241358 & 0.120962 & 0.126980 & 0.762 & 0.75 & \\
\hline Butano $2^{\circ}$ & -157.245399 & 0.122270 & 0.127353 & 0.749 & 0.75 & \\
\hline Iso-butano $1^{\circ}$ & -157.243199 & 0.120702 & 0.126596 & 0.762 & 0.75 & \\
\hline Iso-butano $3^{\circ}$ & -157.251674 & 0.121130 & 0.127270 & 0.763 & 0.75 & \\
\hline Neo-pentano $1^{\circ}$ & -196.428909 & 0.149486 & 0.156556 & 0.762 & 0.75 & \\
\hline \multicolumn{7}{|l|}{ TS2 } \\
\hline Metano & -189.644941 & 0.039355 & 0.043799 & 1.495 & 0.817 & -791 \\
\hline Etano & -228.839237 & 0.070255 & 0.075358 & 1.501 & 0.827 & -633 \\
\hline Propano $1^{\circ}$ & -268.020834 & 0.099631 & 0.106026 & 1.495 & 0.817 & -791 \\
\hline Propano $2^{\circ}$ & -268.028750 & 0.099297 & 0.105943 & 1.501 & 0.826 & -633 \\
\hline Butano $1^{\circ}$ & -307.203242 & 0.129019 & 0.136682 & 1.507 & 0.827 & -639 \\
\hline Butano $2^{\circ}$ & -307.210876 & 0.128629 & 0.136620 & 1.532 & 0.835 & -556 \\
\hline Iso-butano $1^{\circ}$ & -307.204525 & 0.128684 & 0.136363 & 1.501 & 0.827 & -637 \\
\hline Iso-butano $3^{\circ}$ & -307.219523 & 0.127892 & 0.137178 & 1.535 & 0.836 & -571 \\
\hline Neo-pentano $1^{\circ}$ & -346.385082 & 0.157053 & 0.166092 & 1.509 & 0.824 & -667 \\
\hline \multicolumn{7}{|l|}{ ROO• } \\
\hline Metano & -189.684616 & 0.044565 & 0.048397 & 0.760 & 0.75 & \\
\hline Etano & -228.872799 & 0.074295 & 0.079082 & 0.760 & 0.75 & \\
\hline Propano $1^{\circ}$ & -268.054468 & 0.103772 & 0.109778 & 0.761 & 0.75 & \\
\hline Propano $2^{\circ}$ & -268.061565 & 0.103230 & 0.109213 & 0.761 & 0.75 & \\
\hline Butano $1^{\circ}$ & -307.236511 & 0.133142 & 0.140523 & 0.761 & 0.75 & \\
\hline
\end{tabular}




\begin{tabular}{|l|l|l|l|l|l|l|}
\hline Continuación de la Tabla 3.1 \\
\hline Butano $2^{\circ}$ & -307.244170 & 0.132639 & 0.139920 & 0.761 & 0.75 & \\
Iso-butano $1^{\circ}$ & -307.239039 & 0.132658 & 0.139967 & 0.761 & 0.75 & \\
Iso-butano $3^{\circ}$ & -307.251754 & 0.131619 & 0.138898 & 0.761 & 0.75 & \\
${\text { Neo-pentano } 1^{\circ}}^{\text {Neo-346.426026 }}$ & 0.161285 & 0.169780 & 0.761 & 0.75 \\
\hline
\end{tabular}


Tabla 3.2 Energias en hartrees B3LYP/6-31 $\mathrm{G}^{\star \star}$ (Single Point), valores de $\left\langle\mathrm{S}^{2}\right\rangle$ antes y después de la proyección de los reactivos, productos y estados de transición.

\begin{tabular}{|c|c|c|c|}
\hline Sistema & B3LYP/6-31G** & $\left\langle\mathbf{S}^{2}\right\rangle$ Antes & $\left\langle\mathbf{S}^{2}\right\rangle$ Después \\
\hline $\mathrm{OH} \bullet$ & -75.728419 & 0.752 & 0.75 \\
\hline $\mathrm{H}_{2} \mathrm{O}$ & -76.419702 & 0.0 & 0.0 \\
\hline $\mathrm{O}_{2}$ & -150.318617 & 2.007 & 2.0 \\
\hline \multicolumn{4}{|l|}{$\mathbf{R H}$} \\
\hline Metano & -40.523907 & 0.0 & 0.0 \\
\hline Etano & -80.838539 & 0.0 & 0.0 \\
\hline Propano & -119.155053 & 0.0 & 0.0 \\
\hline Butano & -158.471582 & 0.0 & 0.0 \\
\hline $\begin{array}{l}\text { Iso-butano } \\
\text { Neo-pentano }\end{array}$ & -158.472415 & 0.0 & 0.0 \\
\hline \multicolumn{4}{|l|}{ TS1 } \\
\hline Metano & -116.243579 & 0.756 & 0.75 \\
\hline Etano & -155.566877 & 0.756 & 0.75 \\
\hline Propano $1^{\circ}$ & -194.884827 & 0.756 & 0.75 \\
\hline Propano $2^{\circ}$ & -194.886376 & 0.756 & 0.75 \\
\hline Butano $1^{\circ}$ & -234.201447 & 0.756 & 0.75 \\
\hline Butano $2^{\circ}$ & -234.203996 & 0.756 & 0.75 \\
\hline Iso-butano $1^{\circ}$ & -234.202106 & 0.756 & 0.75 \\
\hline $\begin{array}{l}\text { Iso-butano } 3^{\circ} \\
\text { Neo-pentano } 1^{\circ}\end{array}$ & -234.206021 & 0.756 & 0.75 \\
\hline \multicolumn{4}{|l|}{ Radical } \\
\hline Metano & -39.842774 & 0.754 & 0.75 \\
\hline Etano & -79.164965 & 0.754 & 0.75 \\
\hline Propano $1^{\circ}$ & -118.481079 & 0.754 & 0.75 \\
\hline Propano $2^{\circ}$ & -118.487869 & 0.754 & 0.75 \\
\hline Butano $1^{\circ}$ & -157.797579 & 0.754 & 0.75 \\
\hline Butano $2^{\circ}$ & -157.804049 & 0.754 & 0.75 \\
\hline Iso-butano $1^{\circ}$ & -157.798163 & 0.754 & 0.75 \\
\hline $\begin{array}{l}\text { Iso-butano } 3^{\circ} \\
\text { Neo-pentano } 1^{\circ}\end{array}$ & -157.810434 & 0.754 & 0.75 \\
\hline \multicolumn{4}{|l|}{ TS2 } \\
\hline Metano & -192.172942 & 0.96 & 0.752 \\
\hline Etano & -229.502023 & 0.893 & 0.751 \\
\hline Propano $1^{\circ}$ & -268.817722 & 0.913 & 0.752 \\
\hline Propano $2^{\circ}$ & -268.825407 & 0.926 & 0.752 \\
\hline Butano $1^{\circ}$ & -308.134327 & 0.915 & 0.752 \\
\hline Butano $2^{\circ}$ & -308.141344 & 0.958 & 0.752 \\
\hline Iso-butano $1^{\circ}$ & -308.134005 & 0.931 & 0.752 \\
\hline $\begin{array}{l}\text { Iso-butano } 3^{\circ} \\
\text { Neo-pentano } 1^{\circ}\end{array}$ & -308.148284 & 0.974 & 0.753 \\
\hline \multicolumn{4}{|l|}{ ROO. } \\
\hline Metano & -190.218989 & 0.753 & 0.75 \\
\hline Etano & -229.542099 & 0.753 & 0.75 \\
\hline Propano $1^{\circ}$ & -268.857883 & 0.753 & 0.75 \\
\hline Propano $2^{\circ}$ & -268.863984 & 0.753 & 0.75 \\
\hline Butano $1^{\circ}$ & -308.174989 & 0.753 & 0.75 \\
\hline Butano $2^{\circ}$ & -308.180378 & 0.753 & 0.75 \\
\hline Iso-butano $1^{\circ}$ & -308.175171 & 0.753 & 0.75 \\
\hline $\begin{array}{l}\text { Iso-butano } 3^{\circ} \\
\text { Neo-pentano } 1^{\circ}\end{array}$ & -308.185109 & 0.753 & 0.75 \\
\hline
\end{tabular}


Tabla 3.3 Energias de reacción y energias de activación en, $\mathrm{kcal} / \mathrm{mol}$, para la reacción de abstracción de hidrógeno.

\begin{tabular}{|c|c|c|c|c|c|c|c|c|}
\hline Sistema & $\begin{array}{c}\text { Ea } \\
\text { PMP2 }\end{array}$ & $\begin{array}{c}\text { Ea } \\
\text { DFT }\end{array}$ & $\begin{array}{c}\text { Ea } \\
\exp ^{a}\end{array}$ & $\begin{array}{c}\text { Ea } \\
\text { expp }^{b}\end{array}$ & $\begin{array}{c}\Delta \mathrm{E}_{298} \\
\text { PMP2 }\end{array}$ & $\begin{array}{l}\Delta \mathrm{E}_{298} \\
\mathrm{DFT}\end{array}$ & $\begin{array}{c}\Delta \mathbf{H}_{298} \\
\exp ^{d}\end{array}$ & $\begin{array}{l}\Delta \mathrm{H}_{298} \\
\exp ^{\circ}\end{array}$ \\
\hline Metano & 7.76 & 0.90 & 6.7 & $6.34^{f}$ & -11.39 & & -14.12 & -14.38 \\
\hline Etano & 5.47 & -1.89 & 4.0 & 2.084 & -14.09 & & -18.70 & -21.39 \\
\hline Propano $1^{\circ}$ & 4.66 & -2.69 & 3.2 & $1.64 \mathrm{~g}$ & -14.17 & -12.57 & -19.70 & -21.39 \\
\hline Butano $1^{\circ}$ & 4.58 & -2.75 & & $1.60 \mathrm{~g}$ & -13.39 & -11.83 & -21.30 & \\
\hline iso-butano $1^{\circ}$ & 4.64 & -2.60 & & $1.63 \mathrm{~g}$ & -13.03 & -11.58 & -21.20 & \\
\hline neo-pentano $1^{\circ}$ & 4.16 & & & & -12.34 & & & \\
\hline Propano $2^{\circ}$ & 3.85 & -3.65 & 2.2 & 1.232 & -16.34 & -15.95 & -23.00 & -24.39 \\
\hline Butano $2^{\circ}$ & 3.19 & -4.22 & & 0.912 & -15.92 & -15.66 & -24.80 & -24.39 \\
\hline iso-butano $3^{\circ}$ & 2.41 & -4.94 & & 0.514 & -17.92 & -18.86 & -26.50 & -27.39 \\
\hline
\end{tabular}

aT. N. Truong, and D. G. Truhlar. J. Chem. Phys. 93, 1761-1769 (1990).

bN. M. Donahue, J. G. Anderson, K. L. Demerjian. J. Phys. Chem. 102, 3121, (1998).

cComo no hay cambio de número de moles en la reacción de abstracción de hidrógeno entonces $\Delta \mathrm{E}_{298}=\Delta \mathrm{H}_{298}$.

dD. L. Baulch, M. Bowers, D. G. Malcolm, R. T. Tuckerman. J. Phys. Chem. Ref. Data 15, 465, (1986).

${ }^{\mathrm{e} N}$. Cohen and K. R. Westber. J. Phys. Chem. Ref. Data 12, 531, (1983).

D. D. Davis, S. Fisher and R. Schiff. J. Chem. Phys. 61, 2213, (1974)

sValor estimado

Las energias de activación a nivel UMP2 son positivas y van de $7.76 \mathrm{kcal} / \mathrm{mol}$ para el metano, a $2.41 \mathrm{kcal} / \mathrm{mol}$ para la abstracción de un hidrógeno terciario del iso-butano. A nivel B3LYP, excepto para el metano, todas las energias de activación son negativas, lo cual es incorrecto, pero se sabe que este método en general da barreras de activación demasiado pequeñas. Las tendencias en las energias de activación, sin embargo, para los sistemas estudiados a nivel B3LYP es muy parecida a la observada en los resultados UMP2, donde se optimizó completamente la geometría. Se encuentran valores muy similares para todas las abstracciones de hidrógenos primarios $14.6 \mathrm{kcal} / \mathrm{mol}$ para propano, butano, iso-butano y neo-pentano, y valores un poco más grandes para etano y metano), y para las de hidrógenos secundarios (3.85 para propano, 3.19 para butano). La energía de activación calculada para el hidrógeno terciario del iso-butano es de 2.41 $\mathrm{kcal} / \mathrm{mol}$.

Estos resultados sugieren la posibilidad de hacer algún tipo de escalamiento de los valores ab-initio. De hecho, un gráfico de los valores de la energia de activación experimental reportada por Donahue et al. [99] versus los valores de la energía de activación teórica calculada con UMP2 para el camino más probable de cada alcano (es 
decir, la abstracción de un hidrógeno secundario de propano y butano, la de un hidrógeno terciario de iso-butano) genera una relación lineal:

$$
E_{E x p}=-0.64062+0.49369 E_{P M P 2}
$$

con un coeficiente de correlación de 0.9990, (Figura 3.4).

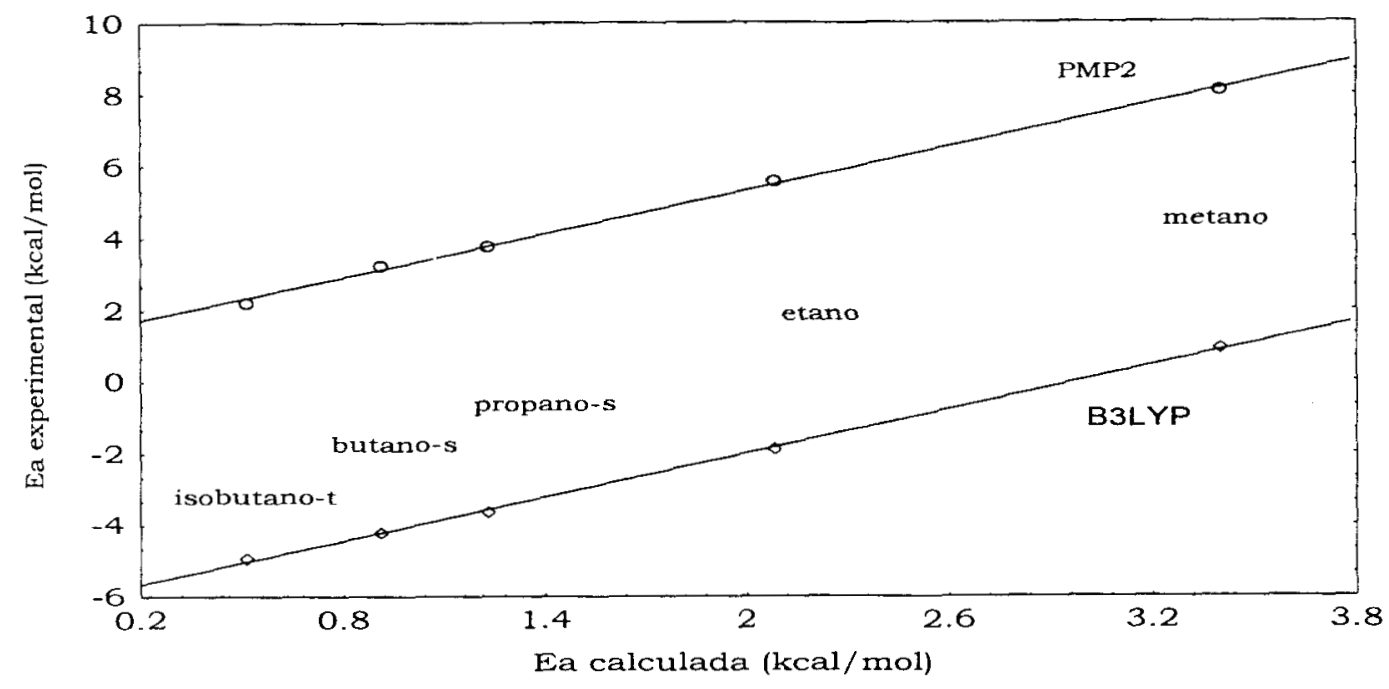

Figura 3.4 Correlación entre las energias de activación experimentales y teóricas.

E1 factor de escalamiento de 0.49 concuerda con lo estimado por Melissas y Truhlar [39], que encontraron que para las reacciones de abstracción de hidrógeno de las parafinas con el radical $\mathrm{OH} \bullet$, el efecto túnel es muy importante en el intervalo de temperatura comprendido entre 300 y $500 \mathrm{~K}$, y que más de la mitad de los eventos que culminan en la formación de productos ocurren por tuneleo. Las energias de activación calculadas para la abstracción de un hidrógeno primario de propano, butano, iso-butano y neo-pentano, para las cuales no hay datos experimentales, son aproximadamente constantes e iguales a $4.60 \mathrm{kcal} / \mathrm{mol}$. Utilizando la ecuación anterior, la correspondiente energia de activación escalada sería de $1.6 \mathrm{kcal} / \mathrm{mol}$.

Un gráfico análogo al anterior pero utilizando las energias de activación calculadas con B3LYP (Figura 3.4) muestra la misma tendencia que con UMP2, con un coeficiente de correlación de 0.9995 y una relación lineal de: 


$$
E_{E x p}=2.98289+0.49057 E_{B 3 L Y P}
$$

En este caso, el factor de escalamiento resulta ser también de 0.49 .

Las únicas parafinas para los cuales se tienen datos de velocidades de reacción para la abstracción de hidrógenos primarios son el metano, el etano y el neo-pentano. Una gráfica de las velocidades de reacción por átomo de hidrógeno primario versus las energias de activación calculadas en este trabajo dá una recta con un coeficiente de correlación de 0.990 (Figura 3.5).

Con respecto a las energias de reacción, utilizando los resultados UMP2 encontramos que en la reacción de formación de un radical $R \bullet$ primario del butano se desprenden $13.5 \mathrm{kcal} / \mathrm{mol}$; mientras que para la formación del radical $\mathrm{R} \bullet$ secundario del butano la energia de reacción es de $-16.0 \mathrm{kcal} / \mathrm{mol}$, y para formarse el radical $\mathrm{R} \bullet$ terciario del iso-butano la energia de reacción es $-18.0 \mathrm{kcal} / \mathrm{mol}$. Con el método B3LYP, para la formación del radical $R \cdot$ primario del butano la energia de reacción es $\Delta E=-12 \mathrm{kcal} / \mathrm{mol}$, para el radical $\mathrm{R} \bullet$ secundario del butano $\Delta \mathrm{E}=-15.7 \mathrm{kcal} / \mathrm{mol}$ y para el radical $\mathrm{R} \bullet$ terciario del iso-butano $\Delta \mathrm{E}=-18.8 \mathrm{kcal} / \mathrm{mol}$. Para los demás parafinas, las energias son aproximadamente constantes aunque cambie el radical $\mathrm{R} \bullet$.

Cabe señalar que, si bien las entalpias experimentales de estas reacciones difieren considerablemente de los resultados teóricos calculados, las tendencias observadas en estos resultados concuerdan muy bien con los resultados experimentales reportados por Baulch et al. [100]. En cambio, los resultados experimentales de Cohen y Westberg [101] muestran una entalpia de reacción a $298 \mathrm{~K}$ que se incrementa dentro de cada grupo conforme aumenta el tamaño de la molécula. En los resultados obtenidos teóricamente, tanto a nivel UMP2 como B3LYP, no se observa esta tendencia. 


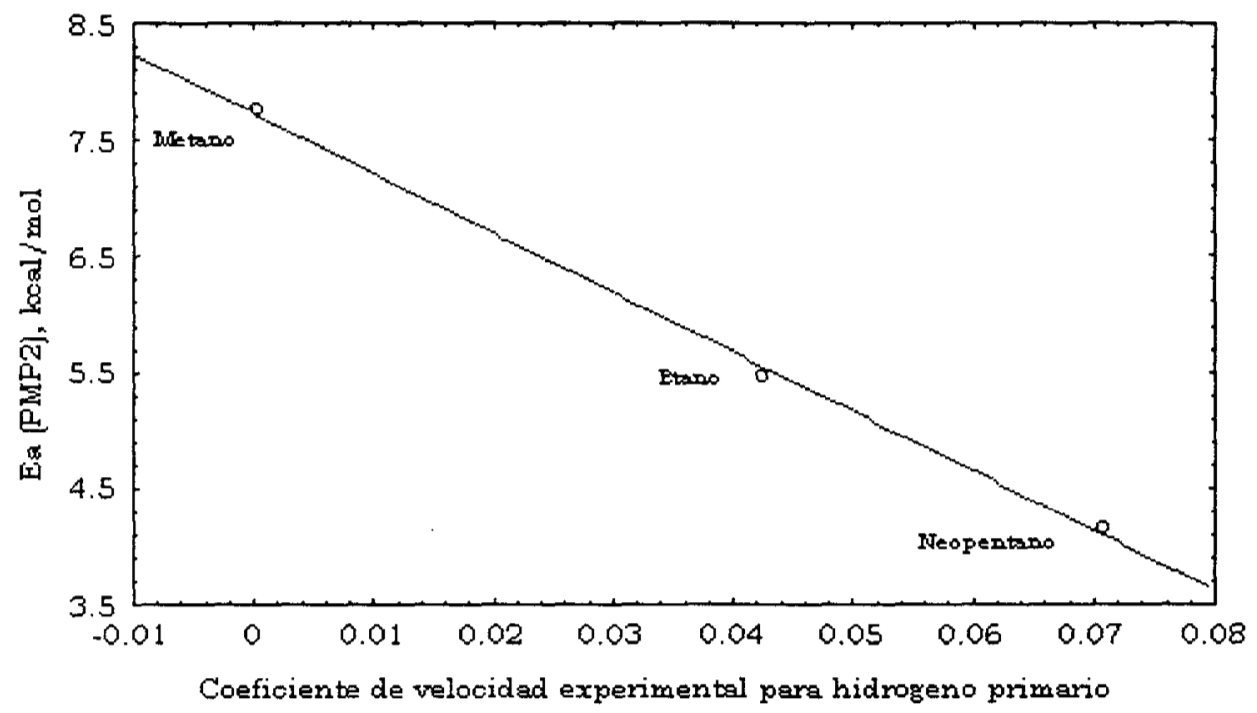

Figura 3.5 Correlación entre las energias de activación teóricas (PMP2) y los coeficientes de velocidad de reacción para hidrógenos primarios.

\subsection{Reacción de adición de $\mathrm{O}_{2}$ en los radicales R•}

Los radicales alquilo al reaccionar con oxigeno molecular forman radicales peroxilo, de acuerdo con la siguiente reaccion:

$$
\mathrm{R} \bullet+\mathrm{O}_{2} \rightarrow \mathrm{RO}_{2} \bullet
$$

Los estados de transición TS2, para la formación de radicales peroxilo $\mathrm{RO}_{2} \bullet$ tanto primarios, como secundarios y terciarios muestran caracteristicas similares. En todas las moléculas diferentes al metano, es decir, en el etano, propano, butano, iso-butano y neopentano, se observa que el $\mathrm{O}_{2}$ se aproxima al hidrocarburo colocándose en una posición aproximadamente paralela al enlace $\mathrm{C}-\mathrm{C}$ y sobre éste. Un átomo de oxigeno interacciona con el átomo de carbono en el que se encuentra el electrón desapareado y el otro átomo de oxígeno interacciona con un átomo de $\mathrm{H}$ del grupo metilo o metileno más cercano, según sea el caso, dando lugar a la formación de un estado de transición TS2 cíclico de cinco miembros. Algunos de éstos están representados en la Figura 3.6. 


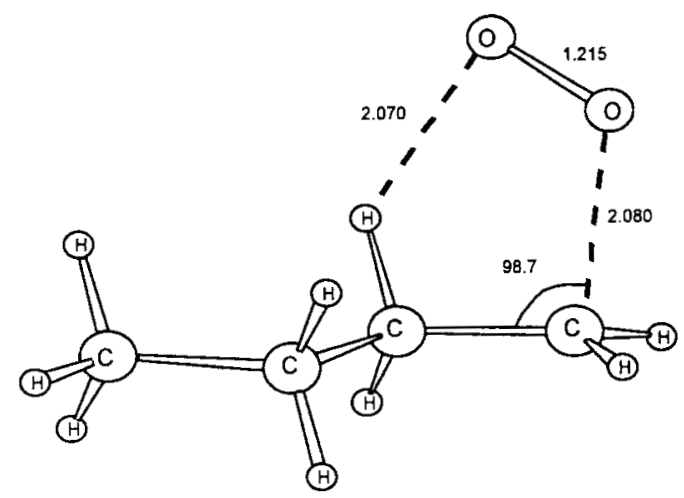

a)

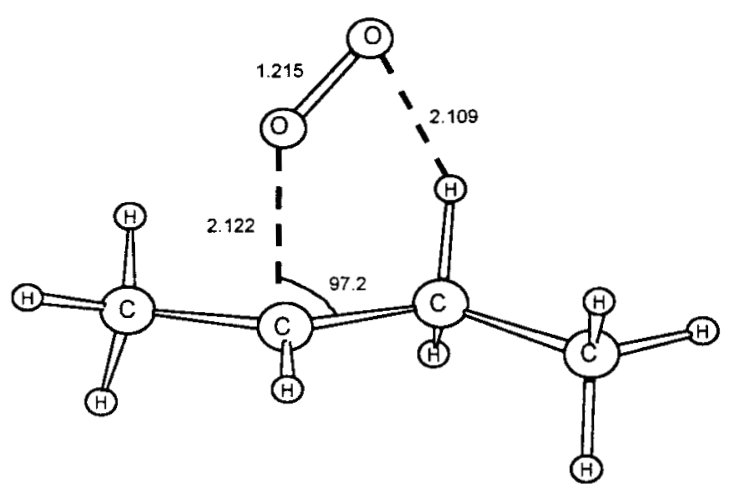

b)

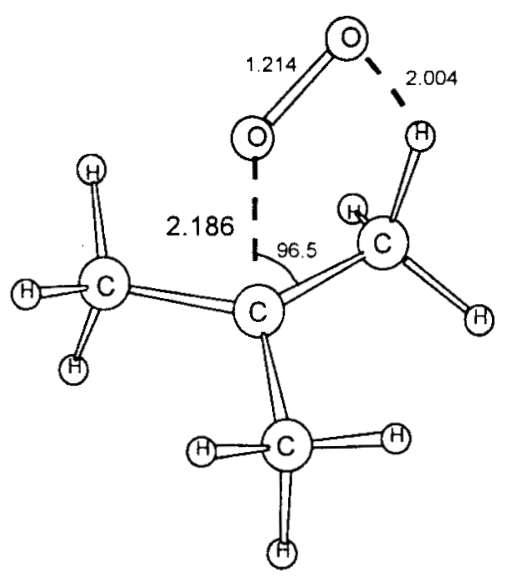

c)

Figura 3.6 Determinación del estado de transición de la reacción de $\mathrm{O}_{2}$ al $\mathrm{R} \bullet$ del butano

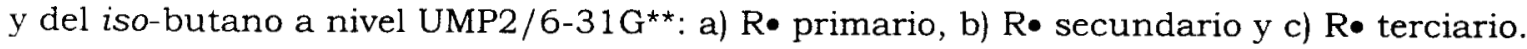


En el TS2, al adicionarse el $\mathrm{O}_{2}$ a un radical R• primario, la distancia C-O es menor $(2.08 \AA)$ que cuando la adición se realiza en un radical secundario $(2.12 \AA)$. Esta distancia $\mathrm{C}-\mathrm{O}$ se incrementa todavia más cuando el $\mathrm{O}_{2}$ se adiciona a un radical terciario $(2.19 \AA)$, indicando que el estado de transición es más temprano cuanto más substituido se encuentra el átomo de carbono.

En la adición de $\mathrm{O}_{2}$ al radical $\mathrm{n}$-butil secundario, un oxigeno interacciona con el carbono donde está el radical y el otro oxigeno tiene, en principio, dos opciones: puede interaccionar ya sea con un hidrógeno del grupo metilo o con un hidrógeno del grupo metileno. Se observa que esta última interacción es la más favorecida energéticamente. El confórmero correspondiente se muestra en la Figura 3.6.

La Figura 3.7 muestra las estructuras optimizadas de los radicales peroxilos $\mathrm{RO}_{2} \bullet$ estudiados. Cuando el $\mathrm{O}_{2}$ está unido al mismo tipo de átomo de carbono, todos los radicales $\mathrm{RO}_{2} \bullet$ presentan caracteristicas similares. Así, por ejemplo, para los radicales $\mathrm{RO}_{2} \bullet$ primarios, el $\mathrm{O}_{2}$ se adiciona siempre paralelo al plano de los carbonos y en posición gauche con respecto a la cadena de carbonos. El átomo de oxigeno externo se encuentra aproximadamente equidistante de los carbonos $\mathrm{C} 1$ y C3. La forma del confórmero más estable para este tipo de radicales $\mathrm{RO}_{2} \bullet$, es similar a la reportada por Ignatyev et al. [87]. Para los radicales peroxilo $\mathrm{RO}_{2} \bullet$ secundarios y terciarios el $\mathrm{O}_{2}$ se adiciona aproximadamente bisectando el ángulo C-C-C. Algunos de los parámetros geométricos de las estructuras de los radicales peroxilo $\mathrm{RO}_{2}$ e estudiados se indican en la Figura 3.7. Para efectos de comparación, la estructura de estos radicales peroxilo $\mathrm{RO}_{2} \bullet$ también se optimizó a nivel B3LYP/6-31G**. Algunos parámetros geométricos calculados a nivel $\mathrm{B} 3 \mathrm{LYP} / 6-31 \mathrm{G}^{\star *}$ se indican entre paréntesis en la misma figura. Como se puede observar, existen solo pequeñas diferencias entre ambos métodos de cálculo.

El análisis de población de Mulliken para los radicales peroxilo $\mathrm{RO}_{2} \bullet$ (Tabla 3.4) utilizando las funciones de onda UHF, UMP2, y B3LYP muestran diferencias considerables entre si. Se observa que las densidades de carga y las de espin calculadas con UMP2 y B3LYP no concuerdan con de las obtenidas con UHF y conducen a conclusiones diferentes. Asi, por ejemplo, en trabajos teóricos previos [3,7,102] sobre propiedades electrónicas de varios radicales peroxilo $\mathrm{RO}_{2} \bullet$, calculadas a partir de densidades $\mathrm{HF}$, se habia establecido que el oxigeno interno llevaba la mayor carga, negativa, del orden del $70 \%$, mientras que el oxigeno externo tenia casi toda la densidad de espin, del orden del $90 \%$. 


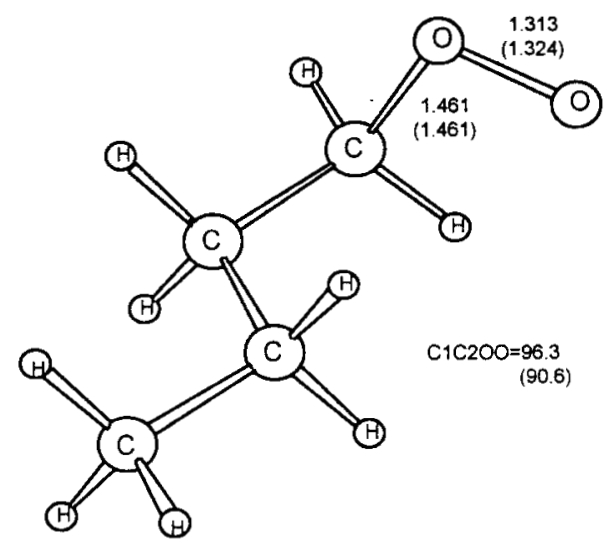

a)

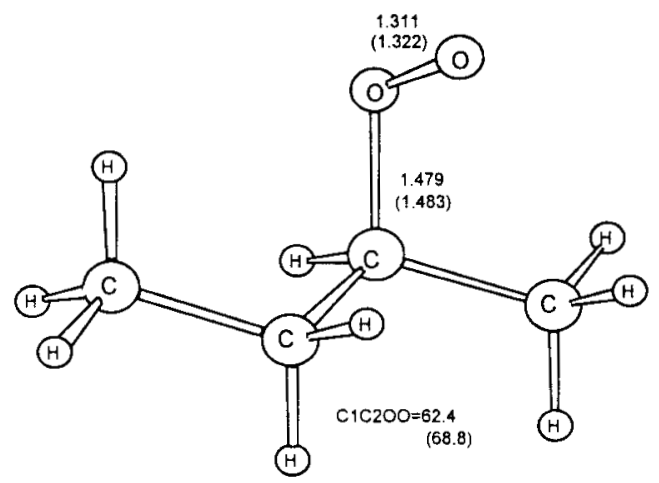

b)

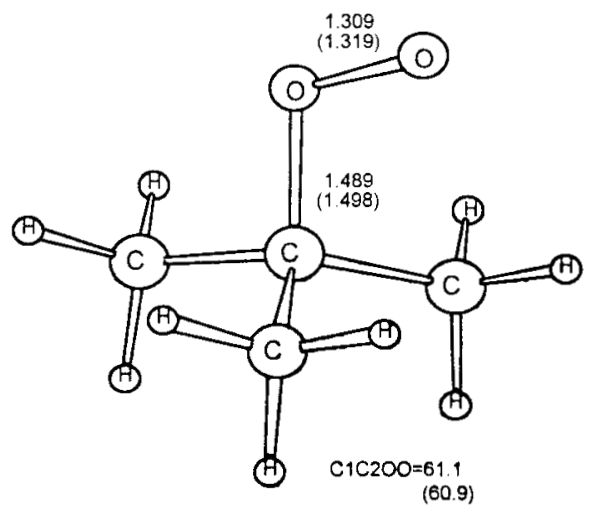

c)

Figura 3.7 Estructuras optimizadas de los radicales $\mathrm{RO}_{2} \bullet$ del butano y del iso-butano a nivel UMP2/6-31G**: a) $\mathrm{RO}_{2} \bullet$ primario, b) $\mathrm{RO}_{2} \bullet$ secundario y c) $\mathrm{RO}_{2} \bullet$ terciario. 
Tabla 3.4 Resultados del análisis de población de Mulliken sobre las cargas y densidades de espin para lo radicales peroxilo del $n$-butano y del iso-butano, a diferentes métodos de cálculo.

\begin{tabular}{|l|l|l|l|l|}
\hline Radical & Propiedad & UHF & UMP2 & B3LYP \\
\hline Butil & \multicolumn{3}{|l|}{} \\
\hline & Carga Oxigeno Terminal & -0.08 & -0.16 & -0.17 \\
& Carga Oxigeno Interno & -0.33 & -0.19 & -0.17 \\
& Densidad de espin Oxigeno Terminal & 0.91 & 0.68 & 0.70 \\
& Densidad de espin Oxigeno Interno & 0.10 & 0.33 & 0.30 \\
\hline Sec-Butil & \multicolumn{3}{|c|}{} \\
\hline & Carga Oxigeno Terminal & -0.08 & -0.17 & -0.17 \\
& Carga Oxigeno Interno & -0.33 & -0.19 & -0.18 \\
& Densidad de espin Oxigeno Terminal & 0.90 & 0.67 & 0.69 \\
& Densidad de espin Oxigeno Interno & 0.10 & 0.34 & 0.30 \\
\hline Tert-Butil & & \multicolumn{3}{|c|}{} \\
\hline & Carga Oxigeno Terminal & -0.08 & -0.16 & -0.17 \\
& Carga Oxigeno Interno & -0.35 & -0.20 & -0.19 \\
& Densidad de espin Oxigeno Terminal & 0.90 & 0.67 & 0.69 \\
& Densidad de espin Oxigeno Interno & 0.10 & 0.34 & 0.30 \\
\hline
\end{tabular}

En estos nuevos resultados, se observa que, si se utilizan densidades UMP2 y B3LYP la carga se distribuye entre los dos átomos de oxígeno y sólo ligeramente en mayor proporción en el oxígeno interno. El átomo de oxigeno interno presenta una carga alrededor de -0.19 y el externo de -0.16. Análogamente, la densidad de espin se distribuye entre los dos oxígenos, aunque se encuentra en mayor proporción en el átomo de oxígeno externo (con 0.67) y en menor proporción en el átomo interno (con 0.33). En la Tabla 3.4 se presentan los resultados del análisis de población de Mulliken para los radicales peroxilo $\mathrm{RO}_{2} \bullet$ del butano y del iso-butano, obtenidos a nivel UMP2 $/ 6-31 \mathrm{G}^{\star *}$.

Las energias totales de los reactivos (los radicales alquilo), los estados de transición (TS2), y los productos (los radicales peroxilo) para esta reacción están reportados en la Tabla 3.1, junto con los valores de $\mathrm{S}^{2}$ antes y después de proyectar el cuarteto. En esta tabla también se reportan las frecuencias imaginarias de los TS2. Se observa que, en los TS2, antes de la proyección hay una grave contaminación de espin, de 1.5 a dos veces mayor que la observada en los TS1. Además, dicha contaminación continúa estando presente aún después de la proyección del estado cuarteto de la función de onda, siendo el valor de $\mathrm{S}^{2}$ alrededor de 0.830 , lo cual indica que también el sexteto contribuye a la función de onda MP2. Esto ocurre siempre que interviene la molécula de oxigeno, ya que el estado fundamental del oxigeno molecular es un triplete que, al combinarse con el doblete del radical alquilo puede dar un doblete y un cuarteto. 
En los TS2 aparece una frecuencia imaginaria mucho más pequeña que en los TS1, entre -571 y $-791 \mathrm{~cm}^{-1}$, dependiendo de la reacción que se trate. La explicación de esto es sencilla. En efecto, las superficies de potencial para esta reacción son mucho más planas que en la reacción anterior. En las reacciones de adición a dobles enlaces, en general, el parámetro de Arrhenius correspondiente a la energia de activación suele ser negativo, lo cual se explica por la formación de un complejo pre-reactivo estable que se forma antes del estado de transición.

En cuanto a los radicales $\mathrm{R} \bullet \mathrm{y} \mathrm{RO}_{2} \bullet$ calculados con el método UMP2/6-31G**, todas las estructuras de mínima energia obtenidas presentan una ligera contaminación de espin con un valor de $\mathrm{S}^{2}$ que va de 0.761 a 0.779 antes de la proyección de la función de onda, y que se elimina totalmente cuando ésta es proyectada.

La Tabla 3.5 muestra las energias de reacción y las energias de activación en $\mathrm{kcal} / \mathrm{mol}$, para la reacción del radical alquílico $\mathrm{R} \bullet \operatorname{con} \mathrm{O}_{2}$, para formar el radical peroxilo $\mathrm{RO}_{2} \bullet$, a nivel UMP2/6-31G**. Se observa que, a excepción del metano cuya energia de activación es de $6.15 \mathrm{kcal} / \mathrm{mol}$, todas las demás reacciones presentan energias de activación negativas. La elevada energia de activación del metano se puede atribuir a que éste no presenta un estado de transición TS2 ciclico, como las demás reacciones, disminuyendo asi su estabilidad.

Las energias de activación de los radicales alquilicos primarios con el $\mathrm{O}_{2}$ a nivel UMP2 $/ 6-31 \mathrm{G}^{\star *}$ son similares, de alrededor de $-1.0 \mathrm{kcal} / \mathrm{mol}$, mientras que para los radicales secundarios es de $-3.8 \mathrm{kcal} / \mathrm{mol}$ y la energia de activación del radical terciario (que por cierto es la más baja) es de $-4.9 \mathrm{kcal} / \mathrm{mol}$. A nivel B3LYP $/ 6-31 \mathrm{G}^{\star *}$ todas las energias de activación son mucho más negativas. Para este último nivel de cálculo, los radicales primarios presentan una energia de activación de $-8.0 \mathrm{kcal} / \mathrm{mol}$, mientras que los radicales secundarios y terciarios presentan una energía de activación muy parecida, de alrededor de $-9.4 \mathrm{kcal} / \mathrm{mol}$.

Las constantes de velocidad de reacción medidas experimentalmente indican que los radicales alquilicos primarios del etano, propano, butano, y neo-pentano reaccionan con $\mathrm{O}_{2}$ a una velocidad de $7.5 \times 10^{-12} \mathrm{~cm}^{-3} \mathrm{~mol}^{-1} \mathrm{~s}^{-1}$, excepto el radical 2-metil-1-propil o isobutil cuya velocidad es más pequeña, del orden de la del metano. El valor de la energia de activación para este radical, obtenido teóricamente, sin embargo, es similar al de los radicales primarios, lo que implicaria que, para explicar los resultados experimentales, el 
factor pre-exponencial en la ecuación de Arrhenius deberia ser, en este caso, mucho más pequeño. Esto último no parece estar justificado y podria ser un error de los datos experimentales. Cabe señalar que la constante de velocidad experimental del radical 2-metil-1-propil a la que se hace referencia fue obtenida por Wu y Bayes [103], mientras que las de los otros peroxilos fueron obtenidas por Atkinson et al. [104] y por Lenhardt et al. [105] y difieren tanto en el método utilizado como en las condiciones de experimentación.

Si bien las energias de activación teóricas para la adición de oxigeno molecular a los radicales 2 -butil y 2-propil son muy similares entre si, las constantes de velocidad de reacción experimentales difieren significativamente. Una posible explicación de esta discrepancia podria encontrarse en la gran incertidumbre que reportan Atkinson et al. [105] en sus datos.

Con respecto a las energias de reacción para la formación de los radicales peroxilo $\mathrm{RO}_{2} \bullet$, los valores calculados son muy parecidos cuando la adición se lleva a cabo en radicales del mismo tipo, y la exotermicidad se incrementa conforme aumenta el número de substituyentes en el átomo de carbono. Los calores de reacción $\Delta \mathrm{H}_{298}$ obtenidos tanto a nivel PMP2 como B3LYP, concuerdan con los resultados experimentales reportados. Estos últimos son pocos, sin embargo, porque los calores de formación de los radicales alquilo primarios, en general no están reportados. Utilizando los resultados teóricos es posible predecir que el calor de reacción para la adición de $\mathrm{O}_{2}$ a los radicales 1-propil, 1 butil y 2-metil-1-propil, deberia ser aproximadamente $-35 \mathrm{kcal} / \mathrm{mol}$, que es el valor del calor de formación del radical etil peroxilo (ver Tabla 3.5). 
Tabla 3.5 Energias de reacción y energias de activación en, kcal/mol, para la reacción de adición de $\mathrm{O}_{2}$ al radical alquílico $\mathrm{R} \bullet$.

\begin{tabular}{|c|c|c|c|c|c|c|}
\hline Sistema & $\begin{array}{c}\text { Ea } \\
\text { (PMP2) }\end{array}$ & $\begin{array}{c}\mathbf{E a} \\
\text { (DFT) }\end{array}$ & $\begin{array}{c}\text { Ea } \\
\text { Exp }\end{array}$ & $\begin{array}{c}\Delta \mathbf{E}_{298} \\
\left(\mathbf{P M P 2}^{\mathrm{a}}\right.\end{array}$ & $\begin{array}{l}\Delta \mathbf{E}_{298} \\
(\mathrm{DFT})\end{array}$ & $\begin{array}{r}\Delta \mathbf{H}_{298} \\
\text { exp }^{\mathbf{b}}\end{array}$ \\
\hline $\begin{array}{l}\text { Metano } \\
\text { Etano } \\
\text { Propano } 1^{\circ} \\
\text { Butano } 1^{\circ} \\
\text { iso-butano } 1^{\circ} \\
\text { Neo-pentano } 1^{\circ}\end{array}$ & $\begin{array}{l}6.15 \\
-0.84 \\
-0.50 \\
-1.48 \\
-0.86 \\
2.22 \\
\end{array}$ & $\begin{array}{l}-8.02 \\
-8.79 \\
-8.18\end{array}$ & $-5.06^{c}$ & $\begin{array}{l}-15.86 \\
-19.56 \\
-19.25 \\
-19.72 \\
-20.26 \\
-21.15 \\
\end{array}$ & $\begin{array}{l}-30.87 \\
-31.75 \\
-31.75\end{array}$ & \begin{tabular}{|l|}
-32.4 \\
-35.2
\end{tabular} \\
\hline $\begin{array}{l}\text { Propano } 2^{\circ} \\
\text { Butano } 2^{\circ}\end{array}$ & $\begin{array}{l}-3.35 \\
-3.78 \\
\end{array}$ & $\begin{array}{l}-9.52 \\
-9.40\end{array}$ & & $\begin{array}{l}-21.89 \\
-22.60 \\
\end{array}$ & \begin{tabular}{|l|}
-31.67 \\
-31.75 \\
\end{tabular} & $\begin{array}{l}-38.25 \\
-38.54\end{array}$ \\
\hline iso-butano $3^{\circ}$ & -4.86 & -9.35 & & -24.01 & -31.38 & \\
\hline
\end{tabular}

a Para la reacción de adición de $\mathrm{O}_{2}$ al radical $\mathrm{R} \bullet, \Delta \mathrm{E}_{298} \cong \Delta \mathrm{H}_{298}+0.6$

b P. D. Lightfoot, R. A. Cox, J. N. Crowley, M. Destriau, G. D. Hayman, M. E. Jenkin, G. K. Moortgat, F. Sabel. Atmos. Environ. 26A, 1805, (1992).

c T. M. Lenhardt, C. E. McDade and K. D. Bayes. J. Chem. Phys. 72, 304, (1980). 


\section{CAPITULO 4}

\section{RESULTADOS Y DISCUSION REACCIONES DE OXIDACIÓN DEL TOLUENO}

\subsection{Las reacciones del tolueno con el radical $\mathrm{OH} \bullet$}

En este capitulo, reportamos las estructuras y energias de los reactivos, productos y estados de transición de las reacciones de oxidación del tolueno. En una primera etapa se consideraron, la reacción de abstracción de un hidrógeno del grupo metilo por el radical $\mathrm{OH} \bullet \mathrm{y}$ la adición de éste al anillo aromático. En una segunda etapa se consideraron las reacciones de adición de oxigeno molecular, al radical bencilo y arilo para formar de los radicales peroxilos correspondientes. Y finalmente, en una tercera etapa se consideraron la ciclización de los radicales peroxilo y formación de radicales epóxidos a través de los aductos biciclos. Los resultados obtenidos a nivel UMP2/6$31 \mathrm{G}^{* *}$ se presentan la Tabla 4.1 .

Tabla 4.1 Energias PMP2 en hartrees, correcciones de vibración en el punto cero (ZPE), correcciones térmicas (TE), valores de $<\mathrm{S}^{2}>$ antes y después de la proyección de la función de onda.

\begin{tabular}{|c|c|c|c|c|c|c|}
\hline Sistema & $\begin{array}{l}\text { PMP2/ } \\
\text { 6-31G** }\end{array}$ & ZPE & TE & $\begin{array}{c}\mathbf{S}^{2} \\
\text { Antes }\end{array}$ & $\begin{array}{c}\mathbf{S}^{2} \\
\text { Después }\end{array}$ & $\begin{array}{c}\text { Frec. } \\
\text { Imag. } v i\end{array}$ \\
\hline $\mathrm{OH} \bullet$ & -75.533571 & 0.008753 & 0.011113 & 0.755 & 0.75 & \\
\hline $\mathrm{H}_{2} \mathrm{O}$ & -76.219788 & 0.021879 & 0.024714 & 0.0 & 0.0 & \\
\hline $\mathrm{O}_{2}$ & -149.955768 & 0.003211 & 0.005579 & 2.0 & 2.0 & \\
\hline Tolueno & -270.692288 & & 0.135824 & 0.0 & 0.0 & \\
\hline \multicolumn{7}{|l|}{ Abstracción } \\
\hline TS1 $1^{\mathrm{a}}$ & -346.214260 & 0.139228 & 0.146904 & 1.315 & 1.049 & -1914 \\
\hline TS1 $1 \mathrm{~b}$ & -346.209117 & 0.140565 & 0.147858 & 1.159 & 0.889 & -1980 \\
\hline TS1 1c & -346.201474 & 0.143774 & 0.151322 & 0.980 & 0.796 & -1957 \\
\hline Radical Bencilo & -270.043686 & 0.118147 & 0.123735 & 1.309 & 1.031 & \\
\hline \multicolumn{7}{|l|}{ Adición } \\
\hline Pre-reactivo & -346.233604 & 0.139771 & 0.149714 & 0.761 & 0.750 & \\
\hline TS2 ipso & -346.225056 & 0.144722 & 0.152274 & 1.339 & 1.06 & -638 \\
\hline TS2 orto & -346.225670 & 0.144896 & 0.152583 & 1.330 & 1.050 & -649 \\
\hline TS2 meta & -346.223377 & 0.144466 & 0.152366 & 1.342 & 1.060 & -676 \\
\hline TS2 para & -346.223620 & 0.144718 & 0.152574 & 1.338 & 1.060 & -668 \\
\hline Aducto ipso & -346.257167 & 0.145386 & 0.152777 & 1.140 & 0.847 & \\
\hline Aducto orto & -346.256185 & 0.145793 & 0.153357 & 1.137 & 0.846 & \\
\hline Aducto meta & -346.253164 & 0.145487 & 0.153166 & 1.134 & 0.845 & \\
\hline Aducto para & -346.253540 & 0.145561 & 0.153413 & 1.138 & 0.847 & \\
\hline
\end{tabular}


Analizaremos primero los resultados correspondientes a la reacción de abstracción.

\subsubsection{La reacción de abstracción de hidrógeno de tolueno}

En esta reacción, que es muy parecida a la de abstracción de hidrógeno de una parafina (Capitulo 3), el proceso que tiene lugar es el siguiente:

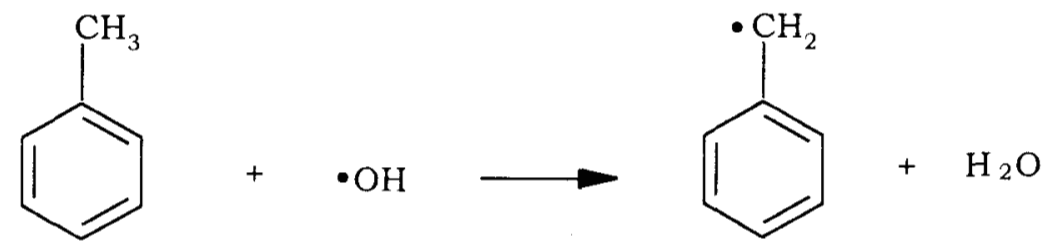

Dado que existe una transferencia de hidrógeno, se siguió la misma metodologia de cálculo que en las reacciones de las parafinas con el radical $\mathrm{OH} \bullet$, es decir que se utilizó el método MP2 con una base de orbitales atómicos que incluye orbitales polarizados sobre los hidrógenos $\left(6-31 G^{\star \star}\right)$. Como se puede observar en la Tabla 4.1, antes de proyectar la función de onda hay una alta contaminación de espín en el estado de transición (TS1), la cual no se elimina totalmente con la proyección del estado cuarteto. Las energias que utilizamos siempre, en este trabajo, son las proyectadas.

En la estructura optimizada del tolueno, el grupo metilo adopta una conformación en la que un enlace $\mathrm{C}-\mathrm{H}$ forma un ángulo recto con el plano del anillo aromático y los otros dos átomos de hidrógeno que se encuentran por debajo del plano del anillo aromático forman un ángulo diedro de $31.3^{\circ}$. La abstracción de estos dos tipos de hidrógeno da lugar a diferentes estados de transición, siendo el primero el de menor energia. El producto, el radical bencilo, es una estructura plana.

En la reacción de abstracción la coordenada de reacción es, esencialmente, la distancia entre el oxigeno del radical $\mathrm{OH} \bullet$ y un hidrógeno del carbono del grupo metilo, a través del puente C---H---O. Existen tres estructuras correspondientes al estado de transición para la reacción de abstracción. En todas ellas se observan caracteristicas parecidas a las de la misma reacción en parafinas, en particular en lo que respecta a la aparición de un estado de transición temprano. En dos de ellas, se trata de la abstracción del hidrógeno del metilo que está en ángulo recto con respecto al plano del 
anillo. La diferencia entre las dos estructuras radica en la orientación del átomo de $\mathrm{H}$ del grupo $\mathrm{OH}$; en una de ellas, este átomo está sobre el anillo aromático, mientras que en la otra el átomo de $\mathrm{H}$ está hacia afuera del anillo. El tercer estado de transición corresponde a la abstracción de un hidrógeno que se encuentra casi en el plano del anillo aromático y es el menos favorable. La conformación más estable es la de la Figura 4.1a con el hidrógeno del grupo $\mathrm{OH}$ orientado sobre el anillo aromático, siendo su energia 0.47 $\mathrm{kcal} / \mathrm{mol}$ menor que la que tiene el grupo $\mathrm{OH}$ orientado hacia afuera.

La estructura 4.1a presenta una distancia $\mathrm{C}-\mathrm{H}$ de $1.19 \AA$, H-O de $1.29 \AA$, y un ángulo de enlace de $163.5^{\circ}$. La distancia entre el átomo de $\mathrm{O}$ del $\mathrm{OH} \bullet \mathrm{y}$ el C es de $2.48 \AA$, caracteristica de un puente de hidrógeno, como ya se había observado en el estado de transición de las parafinas con el radical $\mathrm{OH} \bullet$. Un análisis de población de Mulliken para los átomos que intervienen en la coordenada de reacción, en el estado de transición más estable, muestra las siguientes cargas: -0.392 para el carbono, -0.519 para el oxigeno y +0.292 para el hidrógeno. La carga para el $\mathrm{H}$ del grupo $\mathrm{OH}$, es de +0.324 , lo que permite la interacción entre este hidrógeno muy positivo y la densidad electrónica deslocalizada en el anillo aromático. En cuanto a la densidad de espin, la del átomo de carbono es de 0.306 , la del oxígeno de 0.688 y la del hidrógeno de -0.020 . Esto indica que, en la estructura del estado de transición más estable, la densidad electrónica de espin se encuentra localizada esencialmente sobre el oxigeno y el carbono involucrados en el vector de transición.

En la Tabla 4.2 se muestran las energias de reacción y de activación calculadas a partir de los datos de la Tabla 4.1, tomando en cuenta las correcciones térmicas. También se reportan varios valores obtenidos experimentalmente.

Tabla 4.2 Energias de reacción y de activación en $\mathrm{kcal} / \mathrm{mol}$ para la reacción de abstracción y su comparación con datos experimentales.

\begin{tabular}{|l|l|l|l|l|l|}
\hline \multicolumn{1}{|c|}{ Sistema } & Ea (PMP2) & Ea exp & $\Delta \mathbf{E}_{298}$ (PMP2) & $\Delta$ H298 $_{2}$ exp & $\Delta \mathbf{H}_{298}$ exp \\
\hline Metano & $7.76^{\circ}$ & $3.40^{\mathrm{b}}$ & $-11.39^{\mathrm{a}}$ & $-14.38^{\mathrm{c}}$ & $-14.12^{\mathrm{d}}$ \\
Etano & $5.47^{\circ}$ & $2.08^{\mathrm{e}}$ & $-14.09^{\mathrm{a}}$ & $-21.39 \mathrm{c}$ & $-18.70^{\mathrm{d}}$ \\
Neo-pentano & $4.16^{\mathrm{f}}$ & & $-12.33^{\mathrm{f}}$ & & \\
Tolueno & $7.19^{\mathrm{f}}$ & $0.9 \pm 1.0 \mathrm{~g}$ & -23.6 & & $-31.3^{\mathrm{d}}$ \\
\hline
\end{tabular}

aI. Garcia-Cruz. M. E. Ruiz-santoyo, J. R. Alvarez-Idaboy and A. Vivier-Bunge. J. Comp. Chem. (1999).

${ }^{b}$ D. D. Davis, S. Fischer, and R. Shiff. J. Chem. Phys.8, (1976)

cN. Cohen, and K. R. Westberg. J. Phys. Chem. Ref. Data. 12, 531, (1983).

¿D. L. Baulch, M. Bowers, D. G. Malcolm, R. T. Tuckerman. J. Phys. Chem. Ref. Data. $15,465,(1986)$.

eN. M. Donahue, J. G. Anderson, and K. L. Dermejian. J. Phys. Chem. 102, 3121, (1998).

f. Garcia, V. Uc, R. Alvarez and A. Vivier. Resultados sin publicar, (1999).

gR. A. Perry, R. Atkinson, and J. N. Pitts. J. Phys. Chem. 81, 296, (1977). 
En esta tabla se han incluido los correspondientes resultados del metano, etano y neo-pentano, por ser parafinas que solo tienen hidrögenos primarios, igual que la cadena lateral del tolueno. Se observa que las energias de reacción calculadas concuerdan con las tendencias experimentales, siendo la reacción del tolueno más exotérmica que las de las demás parafinas. Sin embargo, encontramos que la energía de activación del tolueno es significativamente mayor que la esperada, del mismo orden que la del metano, lo cual está en desacuerdo con los datos experimentales. En este trabajo, la conformación del TS1 del tolueno ha sido cuidadosamente verificada, a fin de estar seguros de que se ha obtenido el verdadero estado de transición. Además el vector de transición de las estructuras presentadas en la Figura 4.1 indica claramente que corresponde a los estados de transición buscados.

Considerando que el efecto túnel para la reacción de abstracción de $\mathrm{H}$ del grupo metilo del tolueno por el radical $\mathrm{OH} \bullet$, sea muy importante, igual que en el caso de la abstracción de hidrógeno de las parafinas, y aplicando la relación encontrada en el capitulo anterior, obtenemos una energia de activación para el tolueno de:

$$
E_{\text {exp }}=-0.64062+0.49369 \times 7.19=2.9 \mathrm{kcal} / \mathrm{mol}
$$

la cual es muy cercana al valor experimental.

Analizando las tendencias, se observa que la energia de la barrera calculada se ubica entre la del metano y la del etano, mientras que de acuerdo a los datos experimentales, el hidrógeno del tolueno deberia ser el más fácil de abstraer. Es posible que exista otro mecanismo, diferente a la abstracción directa, por el cual podria formarse el radical bencilo y posteriormente el benzaldehído, que es el que se mide experimentalmente.

\subsubsection{La reacción de adición del radical $\mathrm{OH} \bullet$ al tolueno}

Cuando los reactantes, $\mathrm{OH} \bullet \mathrm{y}$ tolueno, se aproximan uno al otro, se observa primero un descenso de energia, y a una distancia de aproximadamente $2.5 \AA$, se obtiene un minimo poco profundo, cuya energia es casi $5 \mathrm{kcal} / \mathrm{mol}$ menor que la suma de las energias de los reactivos. A esta distancia, el efecto más importante es la atracción coulómbica, por lo que, en esta estructura, a la que llamamos complejo pre-reactivo, el radical hidroxilo se coloca en posición vertical sobre el anillo aromático, con el extremo 
del $\mathrm{H}$ (que es muy positivo) apuntando hacia el anillo. A medida que el grupo $\mathrm{OH}$ se acerca más al anillo, la energia vuelve a aumentar, mientras el grupo $\mathrm{OH}$ gira de manera a colocarse aproximadamente en forma paralela al anillo (TS2), y finalmente forma el enlace entre el átomo de oxigeno y alguno de los carbonos del anillo.

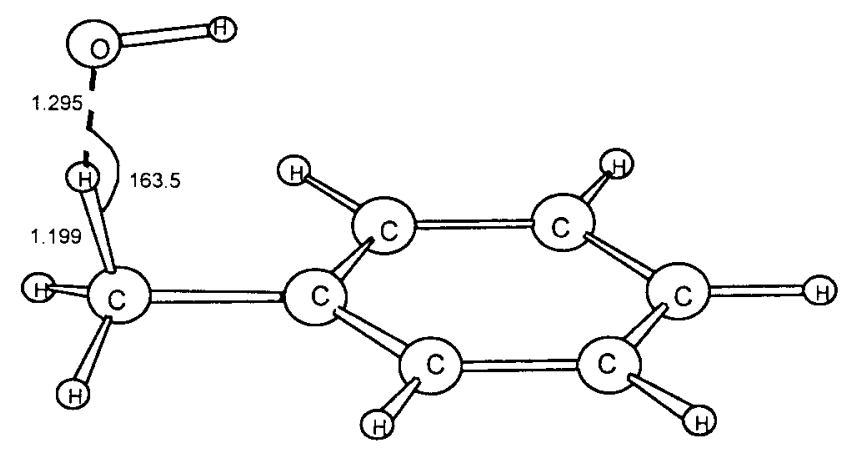

a)

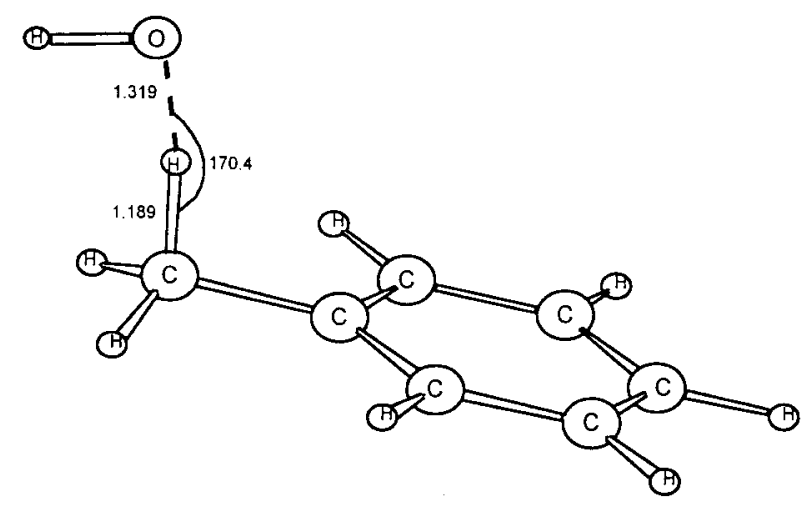

b)

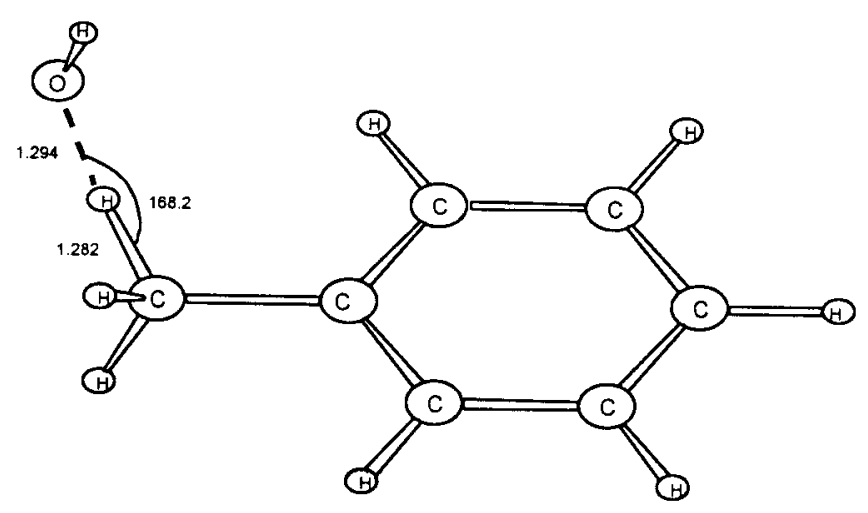

c)

Figura 4.1 Estado de transición del tolueno con el radical $\mathrm{OH} \bullet$, a nivel UMP2/6-31G**. 
En la molécula de tolueno, la adición del radical $\mathrm{OH} \bullet$ al anillo aromático puede, en principio, ocurrir en las posiciones orto, meta y para, y también en la posición ipso, uniêndose al carbono del anillo más cercano al grupo metilo. A partir de un análisis de las concentraciones de la formación de cresol, Kenley et al. [106] establecieron la siguiente selectividad de acuerdo a la de posición de adición:

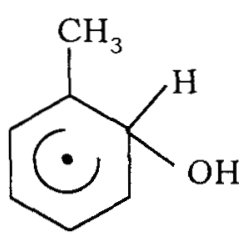

$80.6 \%$

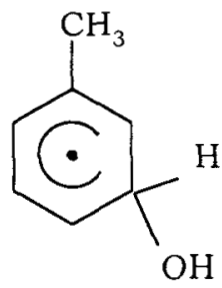

$5.1 \%$

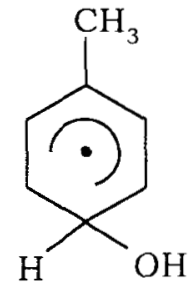

$14.3 \%$

Figura 4.2 Producción de orto, meta y para- cresol a nivel experimetal

Como no se puede obtener un cresol a partir de la adición en ipso, la posibilidad de formar el correspondiente aducto no está contemplada en estos porcentajes.

De acuerdo a reglas bien establecidas de la quimica orgánica para adiciones electrofilicas a anillos aromáticos, se esperaria que la adición del radical $\mathrm{OH} \bullet$ al anillo aromático en el tolueno tuviera lugar en las posiciones orto y para, con una leve preferencia por la posición para debido al impedimento estérico presente en la posición orto. Sin embargo, lo que se observa experimentalmente es que la posición orto es ampliamente favorecida. De hecho, se obtienen entre 5.6 y 15.8 más productos en orto que en las posiciones para y meta, respectivamente. Esta proporción es mucho mayor que la esperada estadisticamente. A partir de los resultados obtenidos en el presente trabajo es posible proponer una explicación.

Las estructuras de los reactivos, de los estados de transición (TS2) y de los aductos $\mathrm{OH}$-tolueno, se optimizaron a un nivel de cálculo UMP2/6-31G**, para las cuatro posiciones posibles de adición. Las correspondientes energias totales, las correcciones térmicas y del punto cero vibracional, el valor medio de $\mathrm{S}^{2}$ antes y después de la proyección y las frecuencias imaginarias en los estados de transición se reportan en la 
Tabla 4.1. En esta tabla, se observa que la contaminación de espin no se elimina totalmente proyectando la función del cuarteto de la función de onda en los TS2 y en los aductos.

En la Tabla 4.3 se muestran las energias de reacción y de activación calculadas y las reportadas experimentalmente. Los valores experimentales se refieren al conjunto de los aductos y no especifican uno en particular.

Tabla 4.3 Energias de reacción y energias de activación en $\mathrm{kcal} / \mathrm{mol}$ para la reacción de adición de $\mathrm{OH}$ al tolueno, y su comparación con datos experimentales.

\begin{tabular}{|l|l|l|l|l|}
\hline Sistema & Ea (PMP2) & Ea expa & $\Delta \mathbf{E}_{\mathbf{2 9 8}}$ (PMP2) & $\Delta \mathbf{H}_{\mathbf{2 9 8}} \mathbf{e x p}^{\mathbf{a}}$ \\
\hline orto-Tol & 0.11 & -1.6 & -19.03 & $-16.5 \pm 5$ \\
ipso-Tol & 0.50 & & -19.65 & \\
para-Tol & 1.40 & & -17.37 & \\
meta-Tol & 1.56 & & -17.13 & \\
\hline
\end{tabular}

aR. A. Perry, R. Atkinson, and J. N. Pitts. J. Phys. Chem. 81, 296, (1977).

De la Tabla 4.3 se observa que el estado de transición orto es el que presenta la energia de activación más baja. A partir de este punto, la continuación de la reacción genera el derivado orto y posteriormente el producto final, el orto-cresol, tal como lo establecen los resultados experimentales reportados en la literatura. El segundo TS en energia, corresponde al de la posición ipso. La causa probable de este ligero incremento en la energia, es el impedimento estérico causado por el grupo metilo. En este caso, el átomo de oxígeno se encuentra más cerca de los hidrógenos del metilo que en la estructura del estado de transición orto-TS y más alejado del carbono reactivo. Los otros dos estados de transición, meta-TS y para-TS son más altos en energia, siendo el estado de transición para-TS ligeramente más bajo que el meta-TS.

El análisis de los resultados PMP2 muestra que ninguno de los cuatro estados de transición descritos previamente presenta una energia más baja que la suma de las energias de los reactivos aislados, de modo que la energía de activación negativa medida experimentalmente no es reproducida mediante estos cálculos. Sin embargo, los resultados PMP2 para los estados de transición orto-TS y ipso-TS presentan energias de activación muy pequeñas, de solo 0.1 y $0.5 \mathrm{kcal} / \mathrm{mol}$, respectivamente [107]. Cuando los cálculos se repitieron con el método B3LYP sí se obtuvieron energias de los TS más bajas que la suma de las energias de los reactivos aislados, aunque éstas son demasiado negativas. Al contrario del método de perturbaciones Møller-Plesset, es bien sabido que el 
método B3LYP tiende a subestimar la altura de las barreras de reacción [4], y que da barreras negativas aún en casos en los que deberian ser positivas.

Con respecto a los aductos, se encontró que el isómero ipso es más estable por 0.5 $\mathrm{kcal} / \mathrm{mol}$ que el isómero orto, si se consideran los valores de energia calculados a nivel PMP2 y PMP4 con la base $6-31 \mathrm{G}^{\star \star}$. Sin embargo, a nivel B3LYP, el isómero ipso es menos estable que los isómeros orto y para. Con la finalidad de resolver esta discrepancia, se realizaron cálculos "single point" con el método Coupled Cluster a partir de las geometrias optimizadas a nivel MP2/6-31G** y B3LYP/6-31G**. El resultado a nivel Coupled Cluster mostró que el isómero ipso es más estable que el isómero orto por $0.25 \mathrm{kcal} / \mathrm{mol}$. Además, en todos los casos, la geometria MP2 resultó ser mucho mejor.

Es interesante hacer notar que las energias de reacción obtenidas a partir de las energias proyectadas PMPn y de los resultados DFT concuerdan con los resultados experimentales. Los resultados Coupled Cluster, sin embargo, muestran valores más bajos, casi a la mitad de los resultados obtenidos con los otros métodos. Hay dos razones que podrian explicar esta discrepancia. Primero, los resultados Coupled Cluster para los aductos presentan una gran contaminación de espín, con $\left\langle\mathrm{S}^{2}\right\rangle=1.13$ y no hay manera de obtener energias proyectadas. Segundo, los cálculos Coupled Cluster se realizan sin optimización de geometria. El interés por realizar cálculos Coupled Cluster en este trabajo es con el fin de ayudar a decidir sobre la estabilidad relativa de los isómeros orto e ipso.

El átomo de oxigeno en el aducto ipso se encuentra a una distancia de $2.35 \AA$ con respecto al átomo de carbono del grupo metilo. La gran estabilidad de este aducto se debe a la interacción de un par de electrones del átomo de oxigeno con los hidrógenos del metilo en una conformación alternada. En el aducto orto, el grupo metilo cambia a una conformación eclipsada a fin de permitir la interacción entre un hidrógeno y el par de electrones del átomo de oxigeno a una distancia de $2.55 \AA$.

La preferencia de la adición del radical $\mathrm{OH} \bullet$ a la posición ipso no es sorprendente. De hecho, es equivalente a la observada en las reacciones de adición del radical $\mathrm{OH} \bullet a$ dobles enlaces, donde la adición se favorece en el carbono más substituido. En el caso del propeno, los cálculos a nivel MP2 [105-106] muestran que el producto de adición más favorecido es aquél en el cual el grupo $\mathrm{OH}$ se adiciona al carbono central. Más recientemente hemos recalculado las energias de los aductos del propeno con el radical 
OH• a nivel B3LYP con optimización completa de la geometría, y, como en el caso del tolueno, la estabilidad de los dos isómeros se invirtió.

Es posible proponer diferentes rutas subsecuentes de reacción para el aducto ipso. Por un lado, se podria formar fenol, siguiendo un camino de oxidación idéntico al del cresol. De hecho, experimentalmente se ha encontrado fenol, aunque en menor cantidad [110]. Otra posibilidad seria que el aducto ipso sufriera un desplazamiento 1,2 del grupo $\mathrm{OH}$, catalizado tal vez por oxigeno molecular, de acuerdo al mecanismo planteado en la Figura 7.3. Esta reacción podría explicar la cantidad de o-cresol, del orden del $80 \%$, medida experimentalmente.
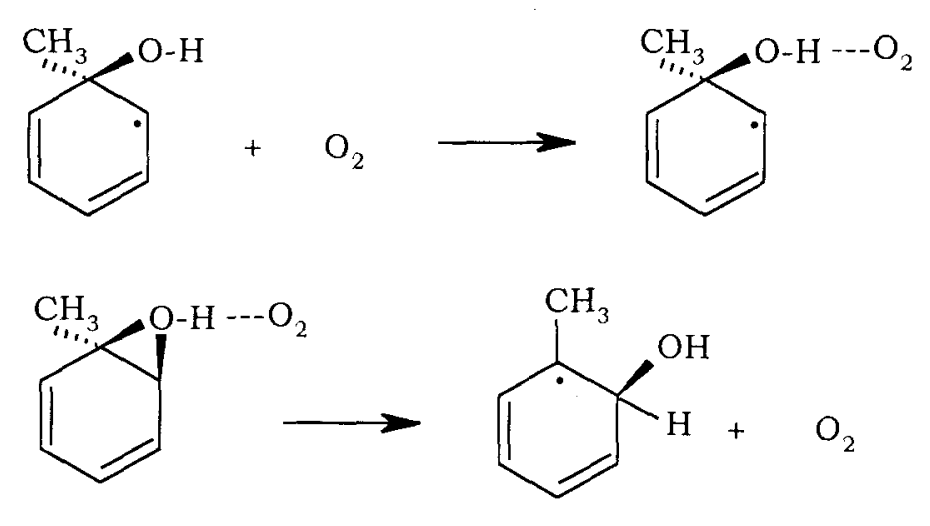

Figura 4.3 Mecanismo de desplazamiento 1,2 del grupo $\mathrm{OH}$ en el aducto ipso para formar orto-cresol.

A partir de la reacción de orto-cresol con $\mathrm{NO}_{2}$, Kenley et al. [106] obtuvieron solamente el 3- $\mathrm{NO}_{2}$-Tolueno. Para esta misma reacción, otros autores [110-112] obtuvieron también pequeñas cantidades de 2 y 4- $\mathrm{NO}_{2}$-tolueno. El 2- $\mathrm{NO}_{2}$-Tolueno podria derivarse del aducto ipso (Figura 4.4). El canal de reacción del aducto ipso para formar el 2- $\mathrm{NO}_{2}$ Tolueno, podria ser más reactivo que el canal de reacción para formar fenol, aunque también podria obtenerse fenol en cantidades muy pequeñas. 

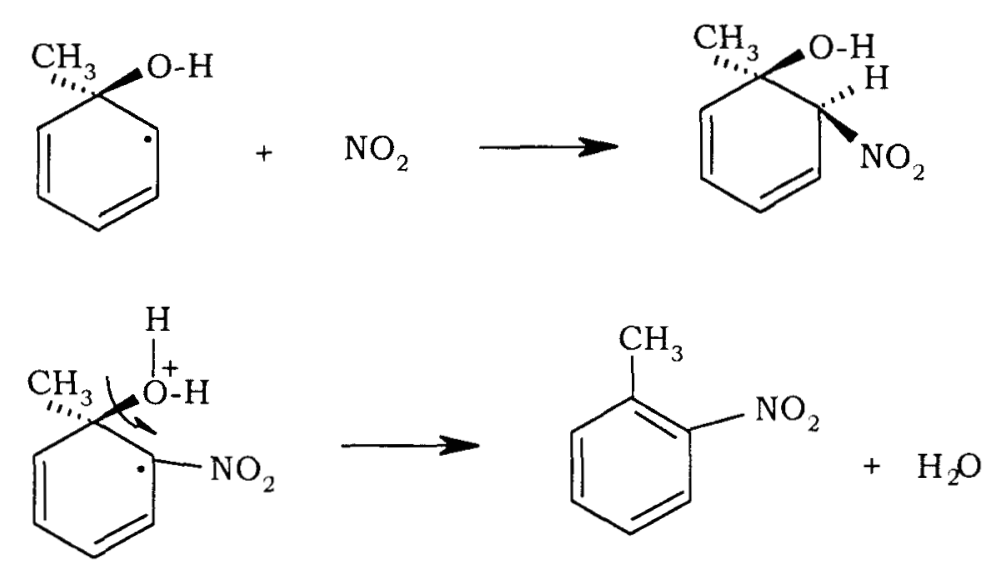

Figura 4.4 Mecanismo de obtención de 2- $\mathrm{NO}_{2}$-Tolueno a partir del aducto ipso.

Aun considerando la posibilidad de que la estabilidad del aducto ipso esté exagerada en los resultados energéticos PMPn y Coupled Cluster, y que las interacciones internas con el par de electrones del átomo de oxigeno estén sobrestimadas, es evidente que la formación del aducto ipso no es despreciable y que debería producir concentraciones detectables de ciertos productos, por lo menos equivalentes a las observadas experimentalmente para meta y para-cresol. Además, el aducto ipso podria generar productos de oxidación derivados de la ruptura del anillo aromático, tales como el metil-glioxal y el butene-dial [113-114], (ver Figura 4.5). Este esquema es similar al propuesto por Shepson et al. [113], para la oxidación del orto-xileno. Los cálculos por orbitales moleculares realizados para los aductos del orto-xileno con $\mathrm{OH} \bullet[115]$, muestran inequívocamente que el aducto ipso es ampliamente favorecido.

Finalmente, se puede sugerir que el aducto ipso también podria estar de algún modo relacionado con la formación de benzaldehido. Este último compuesto se obtiene en cantidades considerables y casi siempre de asume que este compuesto es un producto final de la reacción de abstracción de hidrógeno. Sin embargo, en la sección 4.1 de este Capítulo, se estableció que la barrera para la abstracción directa de un átomo de hidrógeno del grupo metilo del tolueno, para formar el radical bencilo y agua, es alta, del mismo orden de magnitud que la abstracciôn de hidrógeno en el metano. 


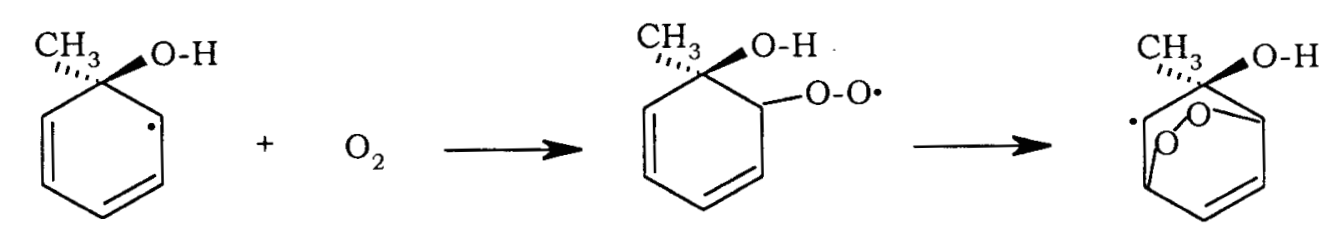

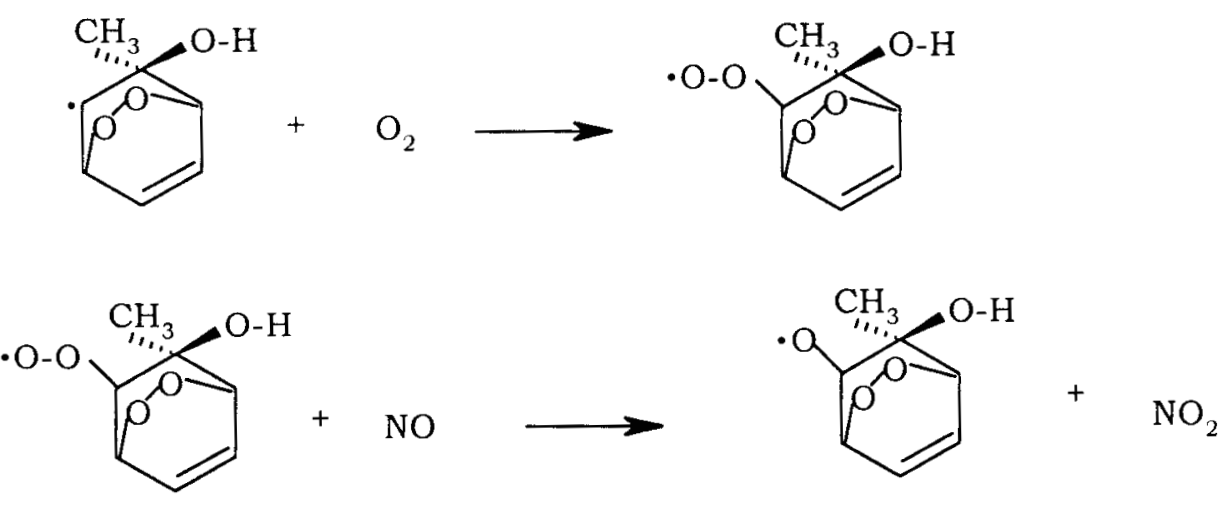

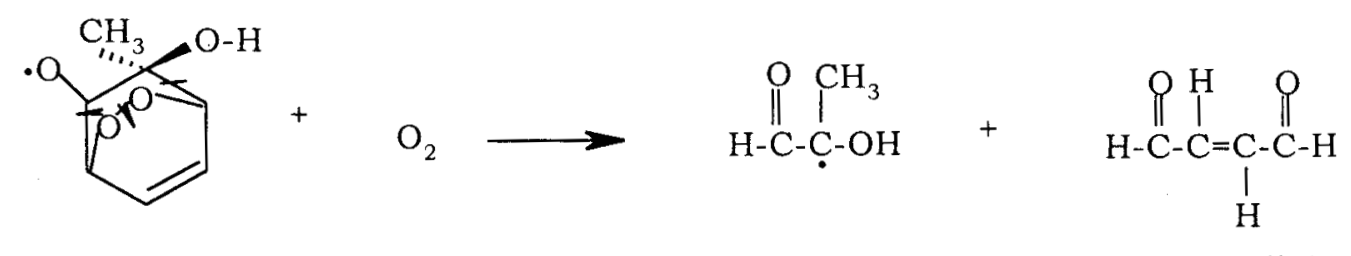
Butedial<smiles>CC(=O)C(=O)CCCCCC(=O)O</smiles>

Figura 4.5 Mecanismo de obtención de los productos butedial y metil-glioxal a partir del aducto ipso.

\subsection{La formación de radicales peroxilos aromáticos}

\subsubsection{La reacción del radical bencilo con $\mathrm{O}_{2}$}

Una vez que se forma el radical bencilo, este puede reaccionar con $\mathrm{O}_{2}$, para formar el radical bencilperoxilo. Esta estructura se estudió en detalle, calculando las barreras de rotación, con respecto al giro alrededor de los diedros D1=CCCO y D2=CCOO (Figura 4.6), a nivel UMP2/631G** $|6|$. Los resultados se muestran en la Tabla 4.4. 


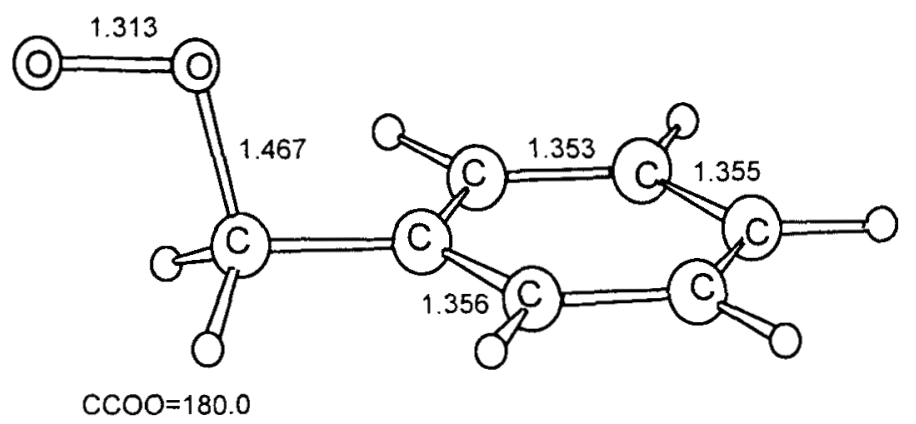

Figura 4.6 Estructura optimizada del radical bencil peroxilo, a nivel UMP2/6-31G**.

Tabla 4.4 Energias en hartrees a nivel UMP2/6-31G** para diferentes conformaciones del radical bencilperoxilo en función de los ángulos diedros D1 y D2. También se indican las energias relativas $\Delta \mathrm{E}$, en $\mathrm{kcal} / \mathrm{mol}$, calculadas con respecto a la estructura más estable (que corresponde a los ángulos diedros D1=90 y D2=180).

\begin{tabular}{|c|l|c|c|c|}
\hline \multicolumn{2}{|c|}{} & \multicolumn{3}{|c|}{$\mathrm{D} 2=\mathrm{CCOO}$} \\
\cline { 3 - 5 } \multicolumn{2}{c|}{} & 0 & 90 & 180 \\
\hline \multirow{3}{*}{$1=\operatorname{CCCO}$} & 0 & -420.024235 & -420.038124 & -420.037647 \\
& & $\Delta \mathrm{E}=10.80$ & $\Delta \mathrm{E}=2.08$ & $\Delta \mathrm{E}=2.38$ \\
\cline { 2 - 5 } & 90 & -420.036445 & -420.040711 & -420.041446 \\
& & $\Delta \mathrm{E}=3.14$ & $\Delta \mathrm{E}=0.46$ & $\Delta \mathrm{E}=0.0$ \\
\hline
\end{tabular}

La Figura 4.6 muestra la estructura más estable, la cual se obtuvo para los ángulos diedros $\mathrm{D} 1=90^{\circ}$ y D2 $=180^{\circ}$. El enlace $\mathrm{O}-\mathrm{O}$ se encuentra en un plano paralelo al del anillo y apuntando hacia afuera, con una conformación trans con respecto al enlace C-C. La molécula de oxígeno se une al orbital semiocupado $2 \mathrm{p}$ del carbono del radical bencilo sin causar mucho cambio en la estructura, excepto el cambio de hibridación en el carbono del metileno.

El cálculo de la curva de potencial para la rotación del grupo C-O-O alrededor del enlace $\mathrm{C}$ - $\mathrm{C}$ de la cadena lateral presenta una barrera de $2.4 \mathrm{kcal} / \mathrm{mol}$. La estructura de máxima energía se obtiene cuando el enlace $\mathrm{O}-\mathrm{O}$ se encuentra hacia el mismo lado y en el plano del anillo aromático, es decir, cuando los ángulos diedros son $\mathrm{D} 1=\mathrm{D} 2=0^{\circ}$. La diferencia de energia con respecto a la conformación más estable es de $10.8 \mathrm{kcal} / \mathrm{mol}$. Las dos conformaciones a $\mathrm{D} 1=90^{\circ}$ y D2 $=0^{\circ}$ y la conformación a $\mathrm{D} 1=0^{\circ}$ y $\mathrm{D} 2=180^{\circ}$ corresponden a puntos de silla, mientras que las otras dos conformaciones, $D 1=\mathrm{O}^{\circ} \mathrm{y}$ $\mathrm{D} 2=90^{\circ}$ y $\mathrm{D} 1=90^{\circ}$ y $\mathrm{D} 2=90^{\circ}$, corresponden a puntos intermedios entre las estructuras de máxima y minima energia. 
Las distancias, C-O de $1.47 \AA$ y O-O de $1.31 \AA$, son muy parecidas a las reportadas anteriormente para radicales peroxilo más pequeños [7,55,116-117]. El orden de enlace de $\mathrm{O}-\mathrm{O}$ calculado en el radical bencil peroxilo es de 0.09 , indicando que es un enlace muy débil y que fácilmente puede reaccionar consigo mismo o con otro radical libre como el NO• [118].

Un análisis de población de Mulliken para estos confórmeros muestra que la carga negativa está repartida entre los dos oxigenos. El átomo de oxigeno interno tiene -0.19 y el externo tiene -0.16. En contraste, la mayor densidad electrónica de espin se encuentra en el oxigeno externo con 0.67 , siendo la del oxigeno interno igual a 0.28 .

El radical bencil peroxilo también presenta un poco de contaminación de espin con un valor de $\mathrm{S}^{2}=0.961$ antes de la proyección de la función de onda, pero al proyectar dicha función la contaminación de espin y el valor de $\mathrm{S}^{2}$ se reduce a 0.785 .

El momento dipolar calculado a nivel MP2 para todos los confórmeros estudiados es del orden de $2.67 \mathrm{D}$, que concuerda con el momento dipolar del radical bencil peroxilo medido experimentalmente [119].

\subsubsection{La reacción del radical $\mathrm{MHCHD} \operatorname{con} \mathrm{O}_{2}$}

Después de formado el aducto OH-tolueno, o MHCHD, se postula que, en la tropósfera, la reacción siguiente es la adición de una molécula de oxigeno molecular para formar el radical $\mathrm{OH}$-tolueno- $\mathrm{O}_{2}$. Atkinson et al. [1] han sugerido que, a continuación, los peroxilos podrian formar un puente O-O a través del anillo, y Edney y Bartolotti [53] han encontrado que también se puede formar un epóxido. Varios isómeros de estos sistemas son posibles. En este trabajo se analizaron veintiocho estructuras del radical $\mathrm{OH}$ tolueno- $\mathrm{O}_{2}$, formadas a partir de la adición de $\mathrm{O}_{2}$ al radical $\mathrm{MHCHD}$ tanto en la posición orto como en la posición ipso.

Los métodos de cálculo que se utilizaron en esta parte del trabajo son el B3LYP/6$31 G^{\star}$ y el UMP2/6-31G*. Fue necesario reducir un poco la base, debido a que los cálculos son muy largos y costosos y se queria analizar muchos isómeros. El uso de la base $6-31 G^{*}$ en vez de la $6-31 G^{\star *}$ utilizada en el caso de las parafinas se justifica porque, en esta reacción, no hay transferencia de hidrógeno, por lo cual usamos funciones de polarización en los átomos pesados pero no en el hidrógeno. En esta etapa, se 
optimizaron y obtuvieron más de veinte estados electrónicos de minima energia, empleando el método de calculo B3LYP/6-31G*. Con el fin de comparar tendencias energéticas, estructurales y electrónicas se optimizaron también algunas estructuras con el método UMP2/6-31G*. En algunos casos este método presentó muchos problemas de convergencia.

La Tabla 4.5 muestra los resultados obtenidos con los dos métodos de cálculo, a nivel B3LYP/6-31G* y el UMP2/6-31G*, para el sistema $\mathrm{OH}$-tolueno- $\mathrm{O}_{2}$ después de la adición del $\mathrm{OH} \bullet$ en posición orto.

El orden de numeración de los átomos en todas las posibles estructuras del

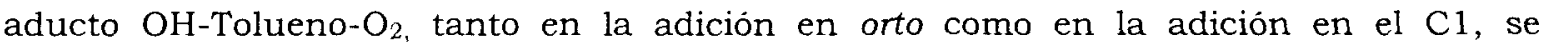
muestra en la Figura 4.7.

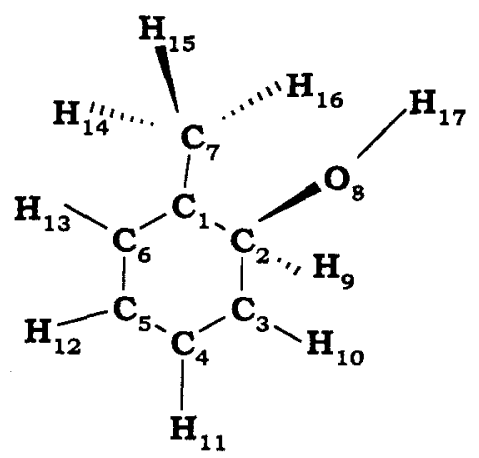

Figura 4.7 Enumeración de los átomos para la optimización de las estructuras

La Figura 4.8 muestra las estructuras optimizadas de los dos aductos iniciales, la adición en orto, o-MHCHD, y la adición en el carbono uno, C1-MHCHD, calculados a nivel B3LYP/6-31G* y UMP2/6-31G*.

En la nomenclatura utilizada en la Tabla 4.5 y en este capitulo, m significa que el $\mathrm{O}_{2}$ y el radical $\mathrm{OH} \bullet$ se adicionaron del mismo lado sobre el plano de anillo aromático, mientras que o significa que el $\mathrm{O}_{2}$ se adicionó del lado opuesto de donde está adicionado el radical $\mathrm{OH} \bullet$.

Como se observa en la Tabla 4.5, las estructuras calculadas a nivel B3LYP/6-31G* casi no presentan contaminación de espín, salvo las estructuras optimizadas para el radical o-MHCHD y para los radicales biciclos VIm y VIo. En contraste, la mayoria de las 
estructuras calculadas a nivel UMP2/6-31G* presentan una alta contaminación, sobresaliendo la de la estructura del radical C1-MHCHD con un valor de $\mathrm{S}^{2}=1.138$ y las estructuras de los radicales biciclos VIs y VIo con un valor de $\mathrm{S}^{2}=0.960$. Esta alta contaminación permanece aún después de la corrección para la estructura del o-MHCHD, con un valor de $\mathrm{S}^{2}=0.837$, mientras que para las estructuras del radical biciclo la contaminación de espin mejora considerablemente obteniéndose un valor de $\mathrm{S}^{2}=0.758$.

Las estructuras de la IIIm a la Vo corresponden a los radicales peroxilo, donde el $\mathrm{O}_{2}$ está adicionado en las posiciones 1,3 y 5 del anillo aromático. La posición 1 , corresponde al carbono donde se encuentra enlazado el carbono del grupo metilo, la posición 3 es adyacente al carbono donde está enlazado el radical $\mathrm{OH} \bullet$ y la posición 5 se encuentra en posición para respecto al $\mathrm{OH} \bullet \mathrm{y}$ meta respecto al grupo metilo.

Las estructuras de la VIm a la XIIo, corresponden a los radicales biciclo, los cuales se forman a través de un puente de dos oxigenos.

Las estructuras de XIm a XIIo corresponden a radicales tipo epóxido, muy estables, que se obtienen una vez que se rompe el puente de oxigenos del radical biciclo más estable (estructura VIm).

La Tabla 4.6 muestra los resultados obtenidos a nivel B3LYP/6-31G* y UMP2/6$31 \mathrm{G}^{*}$ para la adición en el aducto del carbono uno, $\mathrm{C} 1$.

En la Tabla 4.6, se observa que la mayoria de las estructuras calculadas a nivel B3LYP/6-31G* no presentan contaminación de espin, salvo la estructura del C1-MHCHD que muestra una ligera contaminación de espín, con valor de $\mathrm{S}^{2}=0.789$, y la de la estructura XVIm con un valor de $\mathrm{S}^{2}=0.778$. En contraste, la mayoria de las estructuras calculadas a nivel UMP2/6-31G* presentan una alta contaminación de espin, sobresaliendo la del radical C1-MHCHD con un valor de $\mathrm{S}^{2}=1.141$, la del radical peroxilo XIVm con con un valor de $S^{2}=1.995$ y la del radical biciclo XVIm con con un valor de $\mathrm{S}^{2}=0.965$ antes de proyectar la función de onda. Una vez proyectada la función de onda, para el radical C1-MHCHD y el radical biciclo XVIm la contaminación de espin mejora sustancialmente con valores de $\mathrm{S}^{2}$ de 0.848 y 0.759 respectivamente. Sin embargo, para la estructura XIVm la alta contaminación de espin aún permanece con un valor de $\mathrm{S}^{2}=1.552$, después de proyectar la función de onda. 
Tabla 4.5 Energias totales en hartrees a nivel B3LYP/6-31G* y $M P 2 / 6-31 G^{*}$, correcciones en el punto cero (ZPE), correcciones térmicas (TE), valores de $\left\langle\mathrm{s}^{2}\right\rangle$ antes y después de la proyección de onda, para el sistema $\mathrm{OH}-\mathrm{Tolueno- \textrm {O } _ { 2 }}$ en posición orto.

\begin{tabular}{|c|c|c|c|c|c|c|c|}
\hline Radical & $\begin{array}{c}\text { Estruc- } \\
\text { tura }\end{array}$ & B3LYP & $\begin{array}{c}\text { ZPE } \\
\text { (B3LYP) } \\
\text { TE } \\
\text { (B3LYP) }\end{array}$ & $\left\langle\mathbf{S}^{2}\right\rangle$ & PMP2 & $\begin{array}{l}\text { ZPE (HF) } \\
\text { TE (UHF) }\end{array}$ & $\begin{array}{c}\mathbf{S}^{2}> \\
\text { Antes } \\
<\mathbf{S}^{2}> \\
\text { Después } \\
\end{array}$ \\
\hline \multicolumn{8}{|l|}{ O-MHCHD } \\
\hline & & -347.323224 & \begin{tabular}{|l|}
0.141963 \\
0.149656
\end{tabular} & 0.788 & -346.181176 & $\begin{array}{l}0.150849 \\
0.157627 \\
\end{array}$ & \begin{tabular}{|l|}
1.138 \\
0.847 \\
\end{tabular} \\
\hline \multicolumn{8}{|l|}{ Peroxilo } \\
\hline $1-\mathrm{O} 2$ & IIIm & -497.654769 & $\begin{array}{l}0.150764 \\
0.160144\end{array}$ & 0.754 & -496.139526 & $\begin{array}{l}0.161594 \\
0.169604\end{array}$ & $\begin{array}{l}0.762 \\
0.750\end{array}$ \\
\hline $1-\mathrm{O} 2$ & IIIo & -497.650257 & $\begin{array}{l}0.150584 \\
0.160311\end{array}$ & 0.753 & -496.135194 & $\begin{array}{l}0.161666 \\
0.169767\end{array}$ & $\begin{array}{l}0.761 \\
0.750\end{array}$ \\
\hline $3-02$ & IVm & -497.657650 & $\begin{array}{l}0.151088 \\
0.160607\end{array}$ & 0.753 & -496.138669 & $\begin{array}{l}0.161851 \\
0.170012\end{array}$ & $\begin{array}{l}0.762 \\
0.750\end{array}$ \\
\hline $3-02$ & IVo & -497.653913 & $\begin{array}{l}0.150988 \\
0.160856\end{array}$ & 0.753 & -496.125854 & $\begin{array}{l}0.161787 \\
0.169624\end{array}$ & $\begin{array}{l}0.798 \\
0.751\end{array}$ \\
\hline $5-02$ & $\mathrm{Vm}$ & -497.655493 & $\begin{array}{l}0.151493 \\
0.161077\end{array}$ & 0.754 & -496.136221 & $\begin{array}{l}0.162274 \\
0.170519\end{array}$ & $\begin{array}{l}0.778 \\
0.750\end{array}$ \\
\hline $5-\mathrm{O} 2$ & Vo & -497.652758 & $\begin{array}{l}0.150876 \\
0.160762 \\
\end{array}$ & 0.753 & -496.133185 & $\begin{array}{l}0.161960 \\
0.170278 \\
\end{array}$ & $\begin{array}{l}0.774 \\
0.751 \\
\end{array}$ \\
\hline \multicolumn{8}{|l|}{ Biciclo } \\
\hline $1,3-\mathrm{O} 2$ & VIm & -497.668288 & $\begin{array}{l}0.151271 \\
0.159956\end{array}$ & 0.788 & -496.167393 & $\begin{array}{l}0.161692 \\
0.169097\end{array}$ & $\begin{array}{l}0.961 \\
0.758\end{array}$ \\
\hline $1,3-\mathrm{O} 2$ & VIo & -497.664251 & $\begin{array}{l}0.151252 \\
0.159964\end{array}$ & 0.778 & -496.162693 & $\begin{array}{l}0.161783 \\
0.169188\end{array}$ & $\begin{array}{l}0.959 \\
0.758\end{array}$ \\
\hline $1,4-\mathrm{O} 2$ & VIIm & -497.631552 & $\begin{array}{l}0.150376 \\
0.159309\end{array}$ & 0.755 & & & \\
\hline $1,4-02$ & VIIo & -497.629064 & $\begin{array}{l}0.150024 \\
0.159071\end{array}$ & 0.755 & & & \\
\hline $1,5-\mathrm{O} 2$ & VIIIm & -497.636182 & $\begin{array}{l}0.151196 \\
0.159823\end{array}$ & 0.754 & & & \\
\hline $1,5-\mathrm{O} 2$ & VIIIo & -497.631118 & $\begin{array}{l}0.150577 \\
0.159482\end{array}$ & 0.754 & -496.128452 & $\begin{array}{l}0.161992 \\
0.169361\end{array}$ & $\begin{array}{l}0.768 \\
0.750\end{array}$ \\
\hline $3,5-02$ & $\mathrm{IXm}$ & -497.637056 & $\begin{array}{l}0.151536 \\
0.160190\end{array}$ & 0.754 & & & \\
\hline $3,5-\mathrm{O} 2$ & IXo & -497.631770 & $\begin{array}{l}0.150948 \\
0.159877\end{array}$ & 0.754 & -496.126862 & $\begin{array}{l}0.162321 \\
0.169741\end{array}$ & $\begin{array}{l}0.765 \\
0.750\end{array}$ \\
\hline $3,6-02$ & $\mathrm{Xm}$ & -497.632928 & $\begin{array}{l}0.151007 \\
0.160046\end{array}$ & 0.755 & & & \\
\hline $3,6-02$ & Xo & -497.630482 & $\begin{array}{l}0.150970 \\
0.160028\end{array}$ & 0.755 & & & \\
\hline \multicolumn{8}{|l|}{ Epóxido } \\
\hline $\begin{array}{l}\text { 3-0-5,6- } \\
\text { epoxi }\end{array}$ & $\mathrm{XIm}$ & -497.695034 & $\begin{array}{l}0.151347 \\
0.160259\end{array}$ & 0.753 & -496.187522 & $\begin{array}{l}0.162770 \\
0.170478\end{array}$ & $\begin{array}{l}0.776 \\
0.750\end{array}$ \\
\hline $\begin{array}{l}3-0-5,6- \\
\text { epoxi } \\
1-0-3,4- \\
\text { epoxi } \\
1-0-3,4- \\
\text { epoxi }\end{array}$ & $\begin{array}{l}\text { XIo } \\
\text { XIIm } \\
\text { XIIo }\end{array}$ & $\begin{array}{l}-497.686598 \\
-497.691585 \\
-497.681557\end{array}$ & $\begin{array}{l}0.150328 \\
0.159626 \\
0.151698 \\
0.160539 \\
0.150772 \\
0.160100 \\
\end{array}$ & 0.753 & -496.180543 & $\begin{array}{l}0.162220 \\
0.170048\end{array}$ & $\begin{array}{l}0.777 \\
0.750\end{array}$ \\
\hline
\end{tabular}




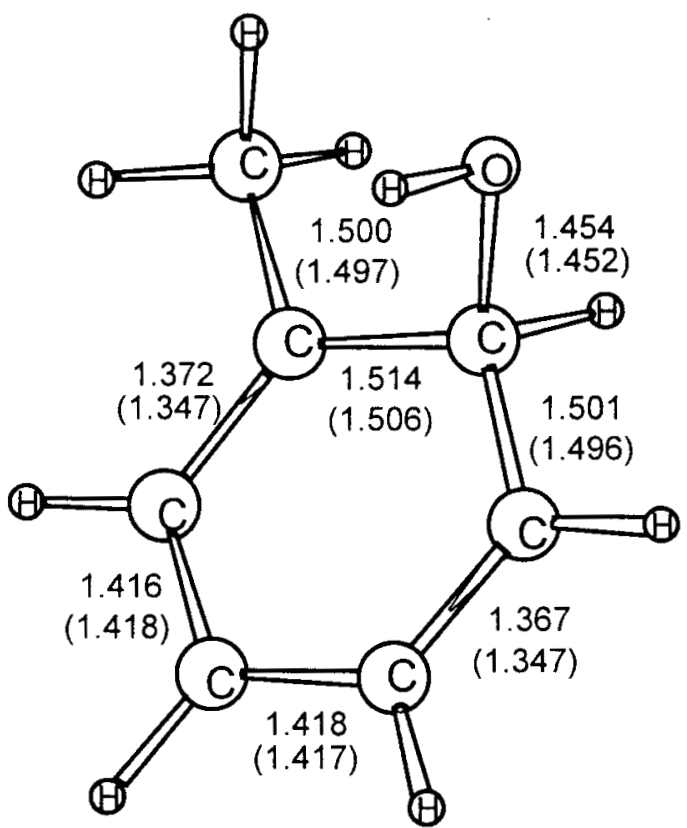

a)

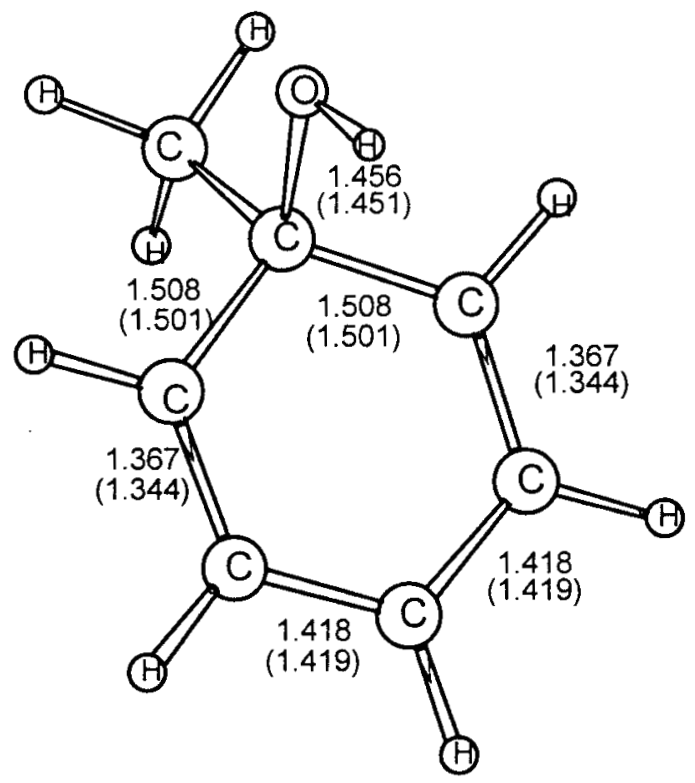

b)

Figura 4.8 Estructuras a) o-MHCHD y b) C1-MHCHD optimizadas a nivel B3LYP/6$31 \mathrm{G}^{\star}$ y UMP2 $/ 6-31 \mathrm{G}^{*}$, cuyos valores están entre paréntesis. 
Tabla 4.6 Energias totales en hartrees a nivel B3LYP/6-31G* y $M P 2 / 6-31 \mathrm{G}^{*}$, correcciones en el punto cero (ZPE), correcciones térmicas (TE), valores de $\left\langle\mathrm{s}^{2}\right\rangle$ antes y después de la proyección de onda para el sistema $\mathrm{OH}$-Tolueno- $\mathrm{O}_{2}$ en el carbono uno, $\mathrm{C} 1$.

\begin{tabular}{|c|c|c|c|c|c|c|c|}
\hline Radical & $\begin{array}{l}\text { Estruc- } \\
\text { tura }\end{array}$ & B3LYP & $\begin{array}{l}\text { ZPE } \\
\text { (B3LYP) } \\
\text { TE } \\
\text { (B3LYP) } \\
\end{array}$ & $\left\langle\mathbf{S}^{2}\right\rangle$ & PMP2 & $\begin{array}{l}\text { ZPE (HF) } \\
\text { TE (UHF) }\end{array}$ & $\begin{array}{l}\mathbf{S}^{2}> \\
\text { Antes } \\
<\mathbf{S}^{2}> \\
\text { Después } \\
\end{array}$ \\
\hline \multicolumn{8}{|c|}{ C1-MHCHD } \\
\hline & & -347.320739 & $\begin{array}{l}0.141575 \\
0.149117\end{array}$ & 0.789 & -346.181945 & $\begin{array}{l}0.150335 \\
0.157028\end{array}$ & $\begin{array}{l}1.141 \\
0.848\end{array}$ \\
\hline \multicolumn{8}{|c|}{ Peroxilo } \\
\hline $\begin{array}{l}2-\mathrm{O} 2 \\
3-\mathrm{O} 2 \\
4-\mathrm{O} 2\end{array}$ & $\begin{array}{l}\text { XIIIm } \\
\text { XIVm } \\
\text { XVm }\end{array}$ & -497.653047 & $\begin{array}{l}0.150652 \\
0.160162 \\
\\
0.151016 \\
0.160464 \\
\end{array}$ & 0.753 & $\begin{array}{l}-496.133434 \\
-496.076269 \\
-496.136319\end{array}$ & $\begin{array}{l}0.161439 \\
0.169461 \\
0.157076 \\
0.165807 \\
0.161837 \\
0.169984 \\
\end{array}$ & $\begin{array}{l}0.762 \\
0.750 \\
1.995 \\
1.552 \\
0.780 \\
0.751 \\
\end{array}$ \\
\hline \multicolumn{8}{|l|}{ Biciclo } \\
\hline $\begin{array}{l}1,6-\mathrm{O} 2 \\
1,4-\mathrm{O} 2\end{array}$ & $\begin{array}{l}\text { XVIm } \\
\text { XVIIm }\end{array}$ & $\begin{array}{l}-497.666013 \\
-497.624659\end{array}$ & $\begin{array}{l}0.151236 \\
0.159854 \\
0.150075 \\
0.158747 \\
\end{array}$ & $\begin{array}{l}0.778 \\
0.753\end{array}$ & $\begin{array}{l}-496.165993 \\
-496.123421\end{array}$ & $\begin{array}{l}0.159885 \\
0.167135 \\
0.160729 \\
0.167987 \\
\end{array}$ & $\begin{array}{l}0.965 \\
0.759 \\
0.762 \\
0.750 \\
\end{array}$ \\
\hline \multicolumn{8}{|c|}{ Epóxido } \\
\hline $\begin{array}{l}\text { 6-O-2,3- } \\
\text { epoxi } \\
4-0-2,3- \\
\text { epoxi } \\
2-0-3,4- \\
\text { epoxi }\end{array}$ & $\begin{array}{l}\text { XVIIIm } \\
\text { XIXm } \\
\text { XXm }\end{array}$ & $\begin{array}{l}-497.691892 \\
-497.689358 \\
-497.686006\end{array}$ & $\begin{array}{l}0.151068 \\
0.159872 \\
0.150705 \\
0.159688 \\
0.150890 \\
0.159783 \\
\end{array}$ & $\begin{array}{l}0.753 \\
0.753 \\
0.754\end{array}$ & $\begin{array}{l}-496.193333 \\
-496.184564 \\
-496.177038\end{array}$ & $\begin{array}{l}0.161541 \\
0.169103 \\
0.162369 \\
0.170063 \\
0.162178 \\
0.169824 \\
\end{array}$ & $\begin{array}{l}0.779 \\
0.751 \\
0.770 \\
0.750 \\
0.759 \\
0.750\end{array}$ \\
\hline
\end{tabular}

Las estructuras de la XIIIm a la XVm corresponden a las de los radicales peroxilo, siendo la estructura XVm la más estable, mientras que la estructura XIVm es la menos favorecida energéticamente.

Las estructuras XVIm y XVIIm corresponde a los radicales biciclos. Mientras que las estructuras XVIIIm, XIXm y XXm corresponden a los radicales tipo epóxido.

La Tabla 4.7 muestra las energias relativas para la reacción de adición en posición orto, a nivel B3LYP/6-31G* y el UMP2/6-31G*. Estas energias fueron calculadas con respecto a la suma de las energias de los reactivos, en este caso el aducto o-MHCHD y el $\mathrm{O}_{2}$. Para las correcciones a la energia, se consideraron únicamente las correcciones térmicas (TE) calculadas a nivel B3LYP/6-31G*. 
La Tabla 4.8 muestra las energias relativas obtenidas a los diferentes niveles de calculo B3LYP/6-31G* y UMP2/6-31G* para la reacción de adición en posición uno, C1. Estas energias fueron calculadas con respecto a la suma de las energias de los reactivos, en este caso el aducto $\mathrm{C} 1-\mathrm{MHCHD}$ y el $\mathrm{O}_{2}$. Para las correcciones a la energia, se consideraron únicamente las correcciones térmicas (TE) calculadas a nivel B3LYP/6$31 \mathrm{G}^{*}$, como en la reacción de adición en posición orto.

Las estructuras más estables de cada grupo, (IIIm, VIm y XIm para la adición en orto, y XVm, XVIm y XIXm para la adición en el carbono uno), se muestran en las Figuras 4.9, 4.10 y 4.11. En cada una de estas figuras se presentan las caracteristicas geométricas más importantes, distancias y ángulos de enlace.

Tabla 4.7 Energias relativas en $\mathrm{kcal} / \mathrm{mol}$ a nivel B3LYP/6-31G* y UMP2/6-31G* para la adición en orto. Las diferencias se obtienen con respecto a la suma de las energias de los reactivos: el radical $\mathrm{MHCHD}\left(\mathrm{E}_{\mathrm{B} 3 \mathrm{LY}}=-347.323224\right.$ hartrees, $\mathrm{TE}_{\mathrm{B} 3 \mathrm{LYP}}=0.149653$ hartrees; $\left.E_{P M P 2}=-346.181177\right)$ y el $\mathrm{O}_{2}\left(E_{\mathrm{B} 3 \mathrm{LYP}}=-150.320038\right.$ hartrees, $T E_{\mathrm{B} 3 \mathrm{LY}}=0.005797$ hartrees; $E_{P M P 2}=-149.955766$ hartrees).

\begin{tabular}{|c|c|c|c|}
\hline Radical & Estructura & B3LYP & PMP2 \\
\hline \multicolumn{4}{|c|}{ Peroxil } \\
\hline & $\begin{array}{l}1-\mathrm{O}_{2} \text { (IIIm) } \\
1-\mathrm{O}_{2} \text { (IIIo) } \\
3-\mathrm{O}_{2} \text { (IVm) } \\
3-\mathrm{O}_{2} \text { (IVo) } \\
5-\mathrm{O}_{2} \text { (Vm) } \\
5-\mathrm{O}_{2}(\mathrm{Vo})\end{array}$ & \begin{tabular}{|l|}
-4.49 \\
-1.56 \\
-6.01 \\
-3.51 \\
-4.36 \\
-2.84 \\
\end{tabular} & $\begin{array}{l}-1.62(-2.39)^{\star} \\
1.10 \\
-1.08(-1-49) \\
8.41 \\
0.45 \\
2.36 \\
\end{array}$ \\
\hline \multicolumn{4}{|c|}{ Biciclo } \\
\hline & $\begin{array}{l}1,3-\mathrm{O}_{2} \text { (VIm) } \\
1,3-\mathrm{O}_{2} \text { (VIo) } \\
1,4-\mathrm{O}_{2} \text { (VIIm) } \\
1,4-\mathrm{O}_{2} \text { (VIIo) } \\
1,5-\mathrm{O}_{2} \text { (VIIIm) } \\
1,5-\mathrm{O}_{2} \text { (VIIIo) } \\
3,5-\mathrm{O}_{2} \text { (Ixm) } \\
3,5-\mathrm{O}_{2} \text { (IXo) } \\
3,6-\mathrm{O}_{2} \text { (Xm) } \\
3,6-\mathrm{O}_{2} \text { (Xo) }\end{array}$ & $\begin{array}{l}-13.09 \\
-10.55 \\
9.55 \\
10.96 \\
6.97 \\
9.93 \\
6.65 \\
9.77 \\
9.15 \\
10.7 \\
\end{array}$ & $\begin{array}{l}-19.11 \\
-16.16 \\
\\
5.33 \\
6.33\end{array}$ \\
\hline \multicolumn{4}{|c|}{ Epóxido } \\
\hline & $\begin{array}{l}\text { 3-O-6,1-ерoxi (XIm) } \\
\text { 3-O-6,1-epoxi (XIo) } \\
\text { 1-O-3,4-epoxi (XIm) } \\
\text { 1-O-3,4-epoxi (XIo) }\end{array}$ & $\begin{array}{l}-29.69 \\
-24.79 \\
-27.35 \\
-21.33 \\
\end{array}$ & $\begin{array}{l}-31.74 \\
-27.36\end{array}$ \\
\hline
\end{tabular}

* Los valores de la energia relativa entre paréntesis se obtuvieron con correcciones térmicas (TE) a nivel UMP2/6-31 $G^{*}$. 
Las estructuras optimizadas para cada grupo de radicales muestran geometrias similares: es decir, todos los radicales peroxilo presentan distancias y ángulos de enlace semejantes, lo mismo sucede para los radicales biciclos, y para los radicales epóxidos. Con base en los resultados obtenidos a nivel B3LYP/6-31G* y UMP2/6-31G*, podemos decir que no se aprecian variaciones importantes en las geometrias calculadas en ambos métodos: de hecho la diferencia en las distancias de enlace es del orden de 0.006 a 0.01 $\AA$, y la diferencia en el ángulo de enlace es menor o igual a un grado.

La Tabla 4.9 muestra las cargas y densidades de espin para la adición en orto, mientras que la Tabla 4.10 muestra las cargas y densidades de espin para la adición en el carbono uno.

Para la adición en orto y para adición en el carbono uno se establece que las estructuras más estables y de minima energia siempre corresponden a los radicales epóxidos [7].

Como se observa en estos resultados, los radicales biciclos son intermediarios importantes, porque a partir de éstos se obtienen los radicales epóxido. En los radicales biciclos la distancia $\mathrm{O}-\mathrm{O}$ es de alrededor de $1.50 \AA$ y la distancia C-O es de $1.46 \AA$, independientemente del método de cálculo utilizado. Debido a que la distancia $\mathrm{O}-\mathrm{O}$ es muy grande, de $1.50 \AA$, este enlace tiende a romperse fácilmente para dar origen a la formación de radicales tipo epóxido, donde la distancia C-O para el enlace tipo epóxido es de $1.43 \AA$ y la distancia C-O del radical libre es de $1.38 \AA$.

En las estructuras más estables, un análisis de población de Mulliken muestra que la carga del $\mathrm{H}$ del radical $\mathrm{OH} \bullet$ exhibe un incremento de +0.40 en los aductos iniciales, a +0.44 en los radicales epóxidos a nivel UMP2 $/ 6-31 G^{*}$, mientras que a nivel B3LYP el incremento es de +0.38 a +0.41 . Cabe señalar que el átomo de carbono donde está enlazado el grupo metilo, en todos los casos presenta una carga positiva de alrededor de +0.50 electrónes (Tablas 4.9 y 4.10 ).

En los radicales peroxilo la carga se encuentra distribuida entre los dos átomos de oxigeno. Así, para el oxigeno interno es de 0.19 y para el oxigeno externo es de 0.16 , mientras que la densidad electrónica de espin se encuentra localizada principalmente en el oxigeno externo con un valor de 0.65 , y en el oxígeno interno es de 0.33. Esto concuerda con estudios recientes para radicales peroxilos, por Wetmore et al. [51] y Garcia-Cruz et al. [6]. 
Tabla 4.8 Energias relativas, en $\mathrm{kcal} / \mathrm{mol}$, a nivel B3LYP $/ 6-31 \mathrm{G}^{*}$ y UMP2/6-31G* para la adición en posición uno, $\mathrm{C} 1$. Las diferencias se obtienen con respecto a la suma de las energias de los reactivos: el radical C1-MHCHD $\left(\mathrm{E}_{\mathrm{B} 3 \mathrm{LY}}=-347.320739\right.$ hartrees, $\mathrm{TE}_{\mathrm{B} 3 L Y \mathrm{P}}=0.149117$ hartrees; $\left.\mathrm{E}_{\mathrm{PMP} 2}=-346.181945\right)$ y el $\mathrm{O}_{2}\left(\mathrm{E}_{\mathrm{B} 3 \mathrm{LY} P}=-150.320038\right.$ hartrees, $\mathrm{TE}_{\mathrm{B} 3 L Y \mathrm{P}}=0.005797$ hartrees; $\mathrm{E}_{\mathrm{PMP} 2}=-149.955766$ hartrees).

\begin{tabular}{|c|c|c|c|}
\hline Radical & Estructura & B3LYP & PMP2 \\
\hline \multicolumn{4}{|l|}{ Peroxil } \\
\hline & $\begin{array}{l}2-\mathrm{O}_{2} \text { (XIIIm) } \\
3-\mathrm{O}_{2} \text { (XIVm) } \\
4-\mathrm{O}_{2} \text { (XVm) }\end{array}$ & $\begin{array}{l}-3.40 \\
-4.43 \\
\end{array}$ & \begin{tabular}{|l|}
2.68 \\
38.55 \\
0.87 \\
\end{tabular} \\
\hline \multicolumn{4}{|l|}{ Biciclo } \\
\hline & $\begin{array}{l}2,6-\mathrm{O}_{2} \text { (XVIm) } \\
2,4-\mathrm{O}_{2} \text { (XVIIm) }\end{array}$ & $\begin{array}{l}-12.95 \\
12.30\end{array}$ & \begin{tabular}{|l|}
-17.75 \\
8.97 \\
\end{tabular} \\
\hline \multicolumn{4}{|l|}{ Epóxido } \\
\hline & $\begin{array}{l}\text { 6-O-2,3-epoxi (XIXm) } \\
\text { 4-O-2,3-epoxi (XVIIIm) }\end{array}$ & $\begin{array}{l}-29.18 \\
-27.71\end{array}$ & $\begin{array}{l}-34.90 \\
-29.40 \\
\end{array}$ \\
\hline & 2-0-3,4-ерохі (XXm) & -25.54 & -24.68 \\
\hline
\end{tabular}




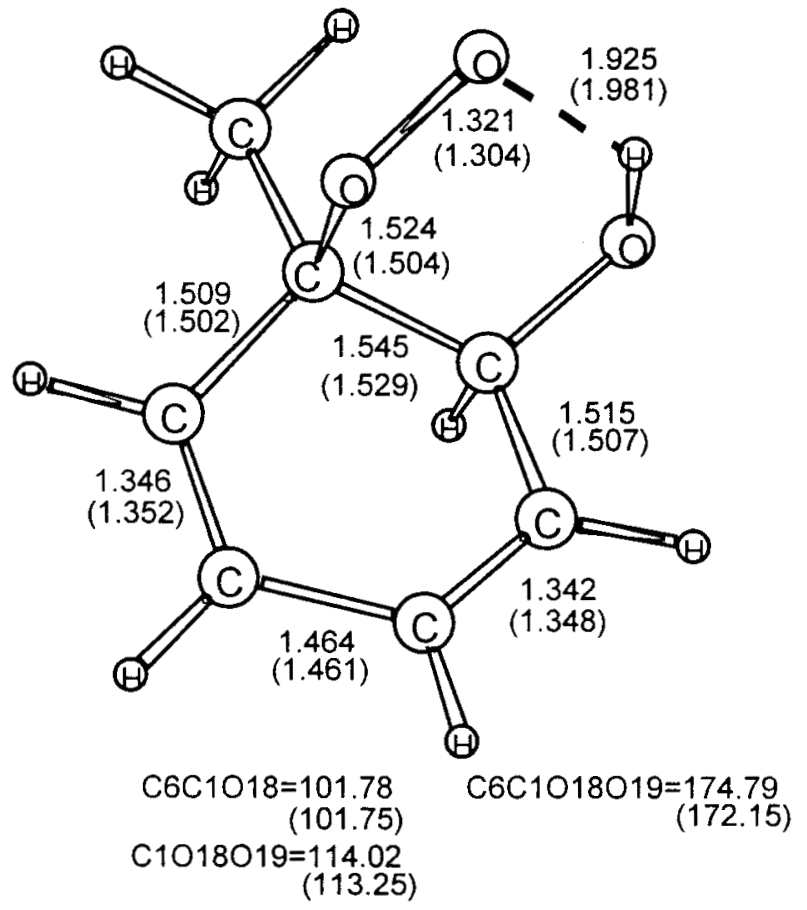

a)

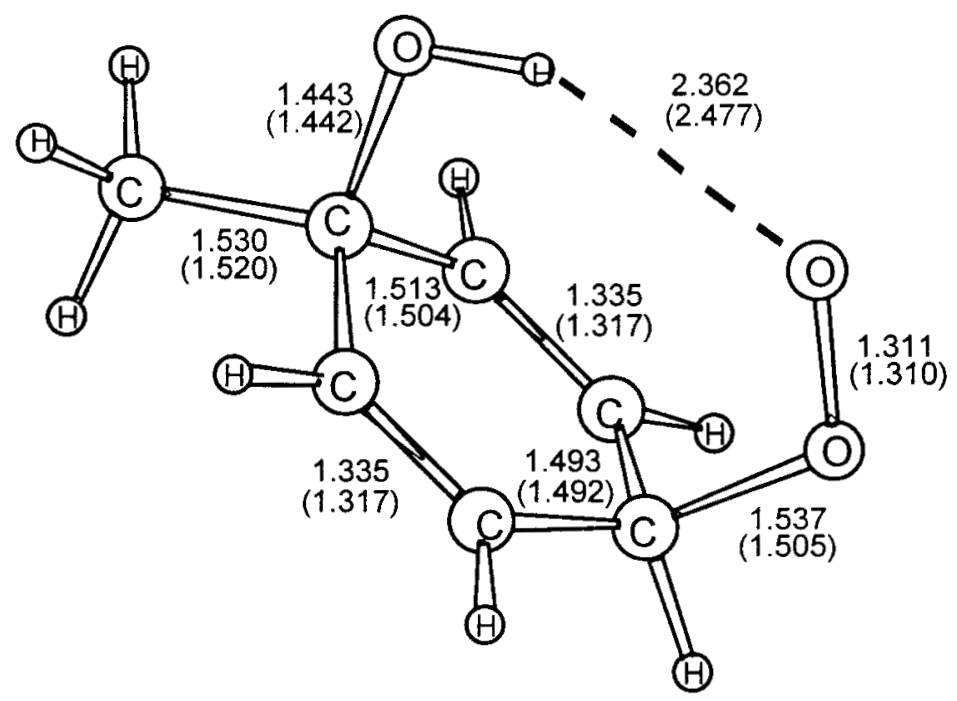

b)

Figure 4.9 Optimización de los radicales peroxilos más estables a nivel B3LYP/6-31G* y UMP2 $/ 6-31 G^{*}$, cuyos valores están entre paréntesis: a) Estructura IIIm y b) Estructura $\mathrm{XVm}$. 

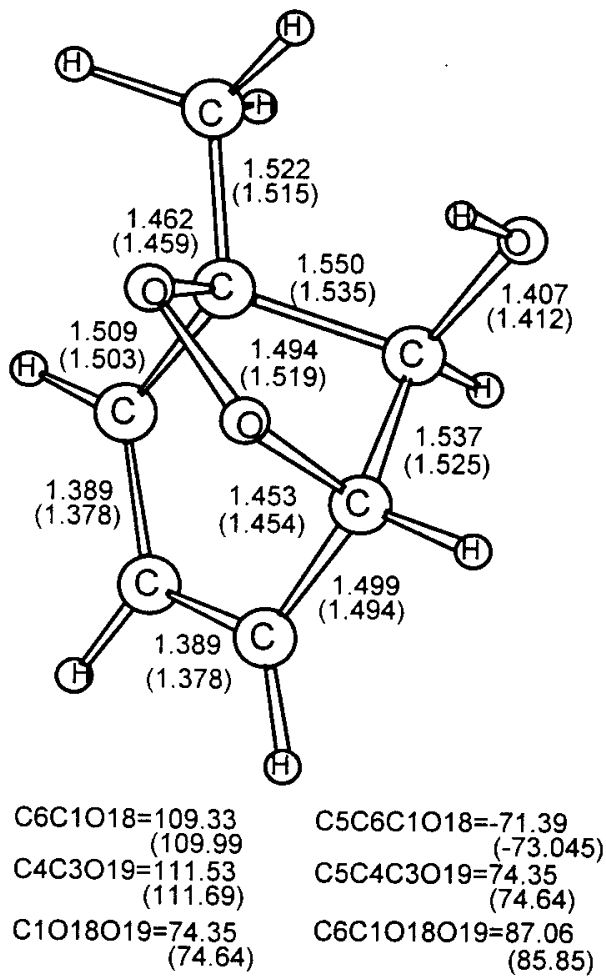

a)

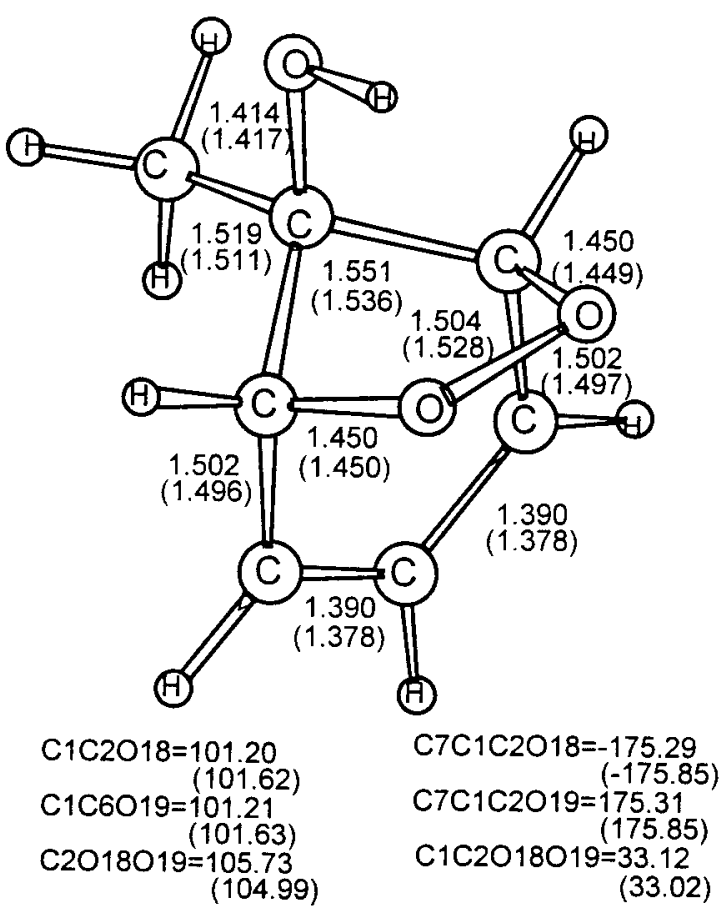

b)

Figura 4.10 Optimización de los radicales biciclos mâs estables a nivel B3LYP/6-31G* y UMP2/6-31G*, cuyos valores están entre paréntesis: a) Estructura VIm y b) Estructura
XVm. 


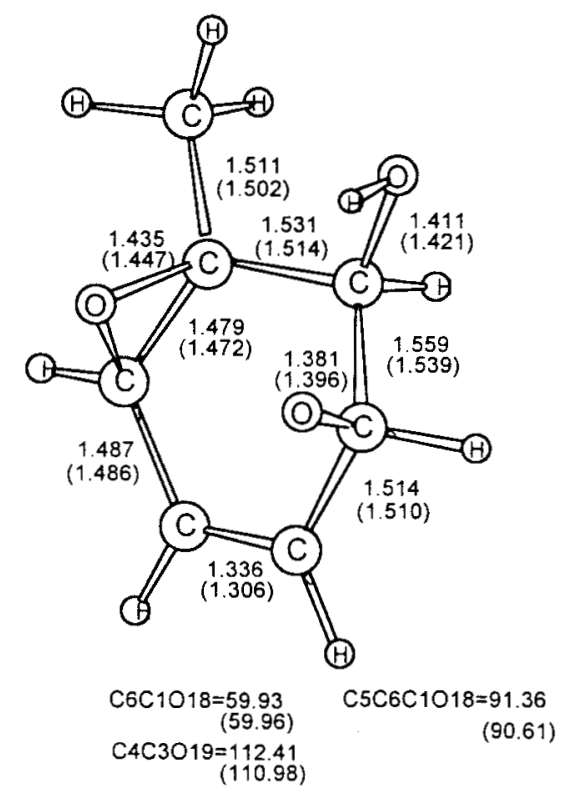

a)

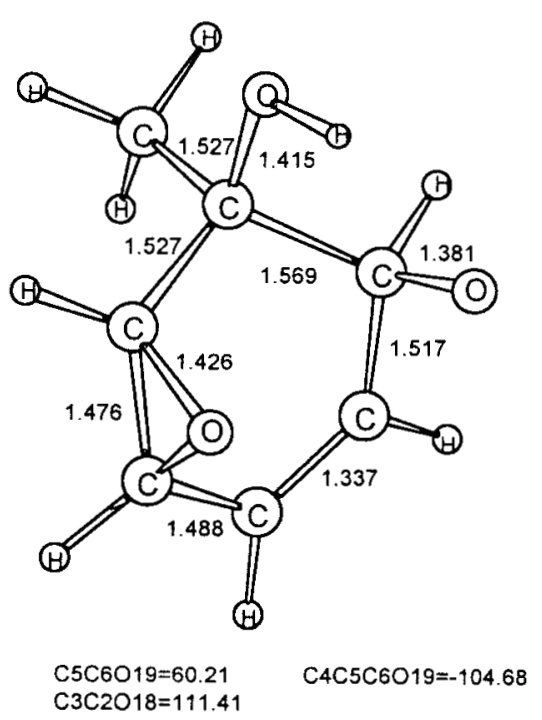

b)

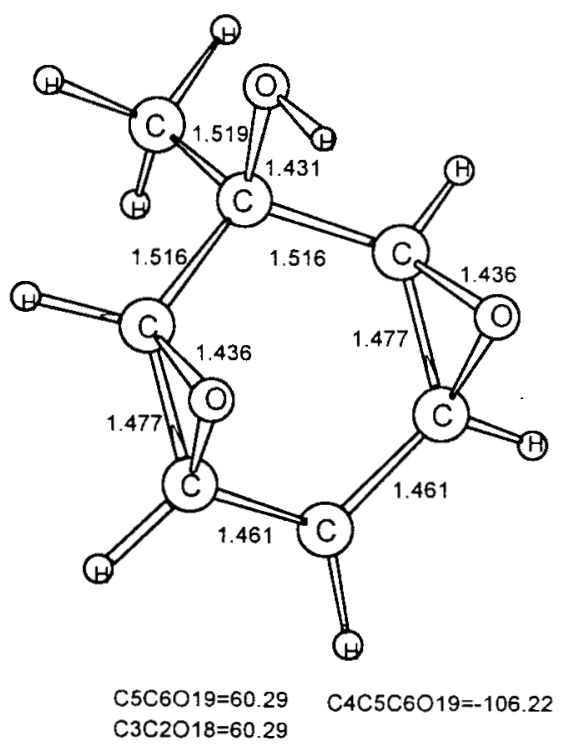

c)

Figura 4.11 Optimización de: a) Estructura XIm a nivel B3LYP/6-31G* y UMP2/6$31 \mathrm{G}^{*}$, b) Estructura XVIIIm a nivel B3LYP/6-31G*, c) Estructura XVIIIm a nivel UMP2/6$31 \mathrm{G}^{*}$. 
Tabla 4.9 Cargas y densidades electrónicas de espin calculadas a nivel B3LYP $/ 6-31 G^{*}$ y el UMP2/6-31G* para la adición en posición orto.

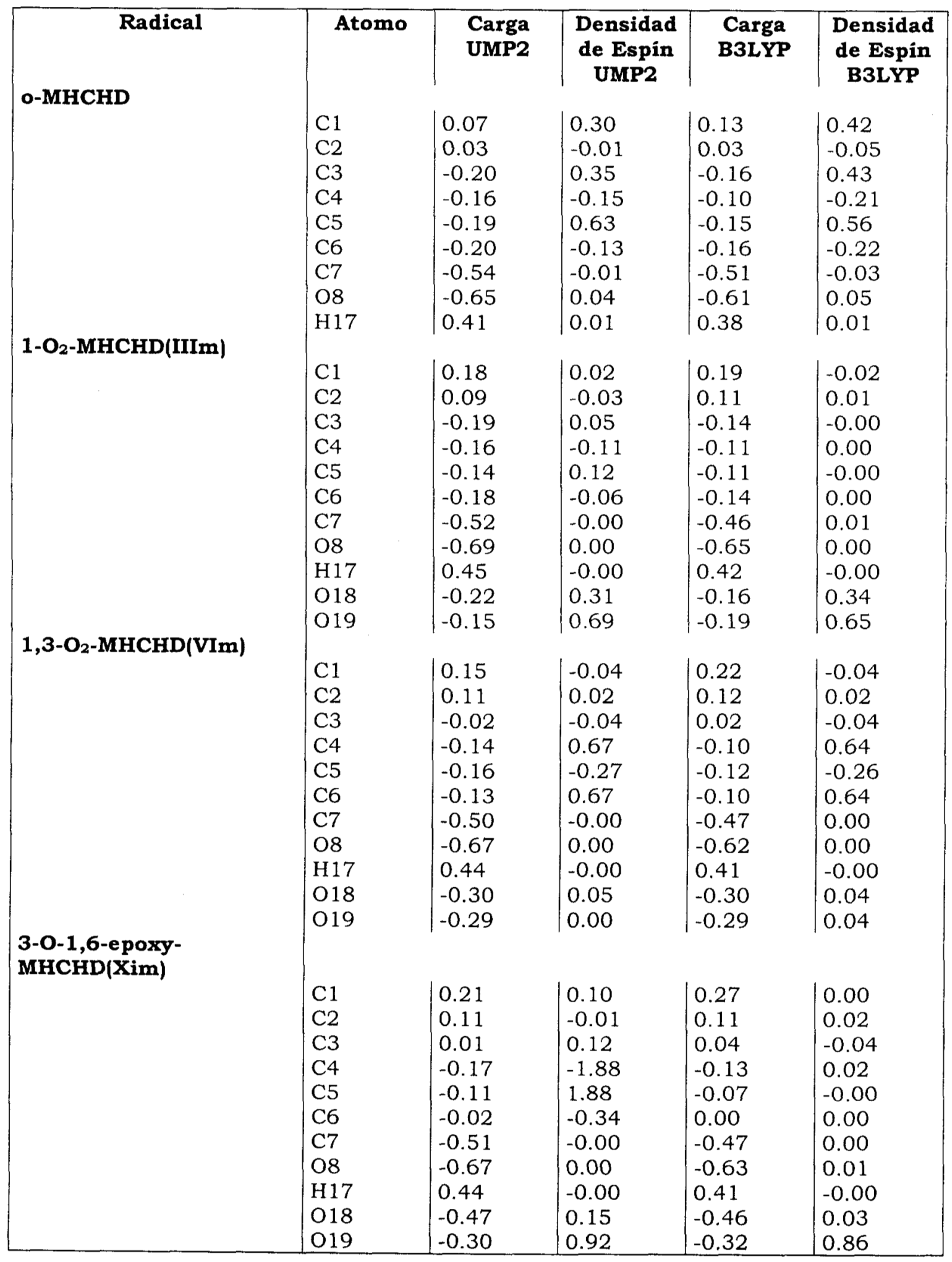


Tabla 4.10 Cargas y densidades electrónicas de espin calculadas a nivel B3LYP/6$31 G^{*}$ y el UMP2/6-31G* para la adición en carbono uno, $\mathrm{C} 1$.

\begin{tabular}{|c|c|c|c|c|c|}
\hline Radical & $\begin{array}{l}\text { Ato } \\
\text { mo }\end{array}$ & $\begin{array}{l}\text { Carga } \\
\text { UMP2 }\end{array}$ & $\begin{array}{c}\text { Densidad } \\
\text { de Espin } \\
\text { UMP2 }\end{array}$ & $\begin{array}{l}\text { Carga } \\
\text { B3LYP }\end{array}$ & \begin{tabular}{|c} 
Densidad \\
de Espin \\
B3LYP \\
\end{tabular} \\
\hline \multicolumn{6}{|c|}{ C1-MHCHD } \\
\hline & $\begin{array}{l}\mathrm{C} 1 \\
\mathrm{C} 2 \\
\mathrm{C} 3 \\
\mathrm{C} 4 \\
\mathrm{C} 5 \\
\mathrm{C} 6 \\
\mathrm{C} 7 \\
\mathrm{O} 8 \\
\mathrm{H} 17\end{array}$ & $\begin{array}{l}0.21 \\
-0.18 \\
-0.16 \\
-0.19 \\
-0.16 \\
-0.18 \\
-0.48 \\
-0.67 \\
0.41\end{array}$ & $\begin{array}{l}-0.00 \\
0.01 \\
-0.00 \\
0.06 \\
-0.00 \\
0.01 \\
0.03 \\
0.03 \\
-0.00\end{array}$ & $\begin{array}{l}0.25 \\
-0.15 \\
-0.11 \\
-0.15 \\
-0.11 \\
-0.15 \\
-0.45 \\
-0.63 \\
0.38\end{array}$ & $\begin{array}{l}-0.06 \\
0.44 \\
-0.21 \\
0.57 \\
0.21 \\
0.44 \\
0.03 \\
0.05 \\
0.00\end{array}$ \\
\hline \multicolumn{6}{|l|}{ 4-O $\mathrm{O}_{2}$-C1MHCHD(XIIIm) } \\
\hline 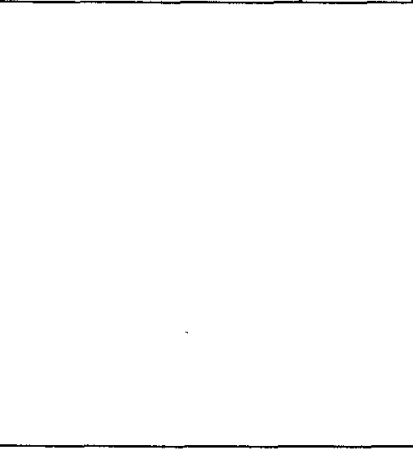 & $\begin{array}{l}\mathrm{C} 1 \\
\mathrm{C} 2 \\
\mathrm{C} 3 \\
\mathrm{C} 4 \\
\mathrm{C} 5 \\
\mathrm{C} 6 \\
\mathrm{C} 7 \\
\mathrm{O} 8 \\
\mathrm{H} 17 \\
\mathrm{O} 18 \\
\mathrm{O} 19\end{array}$ & $\begin{array}{l}0.19 \\
-0.12 \\
-0.17 \\
-0.00 \\
-0.17 \\
-0.12 \\
-0.50 \\
-0.67 \\
0.43 \\
-0.19 \\
-0.15 \\
\end{array}$ & \begin{tabular}{|l|}
-0.35 \\
1.60 \\
1.59 \\
0.32 \\
-1.59 \\
1.16 \\
0.03 \\
0.10 \\
-0.00 \\
0.20 \\
0.69 \\
\end{tabular} & $\begin{array}{l}0.24 \\
-0.09 \\
-0.12 \\
0.01 \\
-0.12 \\
-0.09 \\
-0.46 \\
-0.63 \\
0.40 \\
-0.16 \\
-0.16 \\
\end{array}$ & \begin{tabular}{|l}
0.00 \\
-0.00 \\
0.01 \\
-0.01 \\
0.01 \\
-0.00 \\
-0.00 \\
-0.00 \\
-0.00 \\
0.32 \\
0.68 \\
\end{tabular} \\
\hline \multicolumn{6}{|l|}{ 2,6-O $\mathrm{O}_{2}$-C1MHCHD(XVIs) } \\
\hline & $\begin{array}{l}\mathrm{C} 1 \\
\mathrm{C} 2 \\
\mathrm{C} 3 \\
\mathrm{C} 4 \\
\mathrm{C} 5 \\
\mathrm{C} 6 \\
\mathrm{C} 7 \\
\mathrm{O} 8 \\
\mathrm{H} 17 \\
\mathrm{O} 18 \\
\text { O19 } \\
\end{array}$ & $\begin{array}{l}0.21 \\
-0.01 \\
-0.14 \\
-0.16 \\
-0.14 \\
-0.01 \\
-0.49 \\
-0.68 \\
0.45 \\
-0.30 \\
-0.30 \\
\end{array}$ & \begin{tabular}{|l}
0.02 \\
-0.04 \\
0.67 \\
-0.27 \\
0.67 \\
-0.03 \\
0.00 \\
0.00 \\
-0.00 \\
0.05 \\
0.00 \\
\end{tabular} & $\begin{array}{l}0.29 \\
0.02 \\
-0.09 \\
-0.12 \\
-0.09 \\
0.02 \\
-0.45 \\
-0.64 \\
0.41 \\
-0.29 \\
-0.29 \\
\end{array}$ & $\begin{array}{l}0.03 \\
-0.04 \\
0.63 \\
-0.26 \\
0.63 \\
-0.04 \\
-0.00 \\
0.01 \\
-0.00 \\
0.04 \\
0.04 \\
\end{array}$ \\
\hline \multicolumn{6}{|c|}{ 6-0-2,3-epoxy-C1MHCHD(XVIIIm) } \\
\hline & $\begin{array}{l}\mathrm{C} 1 \\
\mathrm{C} 2 \\
\mathrm{C} 3 \\
\mathrm{C} 4 \\
\mathrm{C} 5 \\
\mathrm{C} 6 \\
\mathrm{C} 7 \\
\text { O8 } \\
\mathrm{H} 17 \\
\text { O18 } \\
\text { O19 } \\
\end{array}$ & $\begin{array}{l}0.27 \\
0.00 \\
0.01 \\
-0.14 \\
0.01 \\
0.00 \\
-0.51 \\
-0.67 \\
0.44 \\
-0.46 \\
-0.46 \\
\end{array}$ & \begin{tabular}{|l|}
-0.00 \\
0.03 \\
-0.07 \\
1.01 \\
-0.07 \\
0.03 \\
-0.00 \\
0.00 \\
0.00 \\
0.06 \\
0.06 \\
\end{tabular} & \begin{tabular}{|l|}
0.29 \\
0.05 \\
-0.12 \\
-0.07 \\
0.02 \\
0.06 \\
-0.46 \\
-0.64 \\
0.41 \\
-0.33 \\
-0.43 \\
\end{tabular} & $\begin{array}{l}0.02 \\
-0.04 \\
0.02 \\
-0.00 \\
-0.00 \\
0.00 \\
-0.00 \\
0.01 \\
-0.00 \\
0.86 \\
0.02 \\
\end{array}$ \\
\hline
\end{tabular}




\subsubsection{Radicales peroxilo}

Los resultados obtenidos para la adición en el aducto orto muestran una tendencia bien marcada: todas las estructuras donde el $\mathrm{O}_{2}$ se adiciona del mismo lado del anillo aromático que el radical $\mathrm{OH} \bullet$, son más estables energéticamente, que aquellas en las que el $\mathrm{O}_{2}$ y el radical $\mathrm{OH} \bullet$ ocupan lados opuestos del anillo aromático.

Una vez que se forman los tres radicales peroxilo, solo hay 5 maneras diferentes de formar radicales tipo biciclo, como lo proponen Atkinson et al [1] y S. Benson [47], aplicando reglas de aditividad de grupos termodinámicos. En la Tabla 4.7 se presentan diez estructuras, debido a que cada una de las estructuras de los 5 biciclos se optimizaron con $\mathrm{O}-\mathrm{O}$ y $\mathrm{O}-\mathrm{H}$ del mismo lado y del lado opuesto del plano del anillo aromático.

La estructura XIm corresponde al radical peroxilo más estable, de acuerdo con lo propuesto por Bartolotti y Edney [53] a través de cálculos teóricos a nivel DFT con un funcional local. Estos resultados también concuerdan con los de Andino et al [54] quienes obtuvieron esta estructura por medio de cálculos teóricos UB3LYP/6-31G*//PM3.

En la adición orto, las estructuras más estables se obtienen al adicionar el $\mathrm{O}_{2}$ y el radical $\mathrm{OH} \bullet$ en el mismo lado del anillo. Siguiendo estas tendencias, para la adición en C1 solo se consideró la adición del $\mathrm{O}_{2}$ en el mismo lado donde está enlazado el radical $\mathrm{OH} \bullet$, por lo que, para este aducto, se calcularon menos estructuras.

La estructura del C1-MHCHD o ipso es simétrica, por lo cual solo hay tres posibilidades de formar un radical peroxilo. Estas corresponden a la adición de $\mathrm{O}_{2}$ en las posiciones 2 o orto, 3 o meta y 4 o para con respecto a la adición del radical $\mathrm{OH} \bullet \mathrm{y}$ al grupo metilo.

Para los radicales peroxilo se observa que, en todas las estructuras en las cuales $\mathrm{O}_{2}$ y $\mathrm{OH}$ - están del mismo lado, hay una fuerte interacción entre el oxígeno terminal del radical peroxilo, $\mathrm{O} 19$, y el $\mathrm{H} 17$ del grupo $\mathrm{OH}$. Esta fuerte interacción se refleja en la distancia que hay entre O19-H17 de $1.92 \AA$ para B3LYP/6-31G* y de $1.98 \AA$ para UMP2 /6-31G* (Figura 4.9). La estabilidad entre los radicales peroxilos del mismo lado y las de lado opuesto oscila entre 1.5 y $3 \mathrm{kcal} / \mathrm{mol}$, dependiendo en que posición se adicione el $\mathrm{O}_{2}$. La diferencia de energia entre los radicales peroxilo más estables es de 
solo $1.5 \mathrm{kcal} / \mathrm{mol}$ (Tabla 4.7 ), lo que indica que a temperatura ambiente, todas estas estructuras podrian formarse fácilmente.

En cuanto a los resultados de los dos métodos de cálculo, hay una discrepancia sobre cual de las estructuras de los radicales peroxilo es la más estable. Según el método B3LYP/6-31G* la adición de $\mathrm{O}_{2}$ en la posición 3 es la más estable: esto concuerda con los estudios teóricos previos, por Bartolotti y Edney [53], y por Andino et al. [54]. En cambio, según UMP2/31G* la adición en la posición 1 (carbono donde está enlazado el grupo metilo) es la más estable. De hecho, en otros estudios que hemos realizado el método UMP2/6-31G* muestra una tendencia muy clara a favorecer la adición de $\mathrm{O}_{2}$ en el carbono más substituido [120], lo cual, en general está de acuerdo con las reglas bien establecidas de la química orgánica.

Como se puede observar en la Tabla 4.7, aunque los dos métodos de cálculo muestran la existencia de estas dos estructuras, sus energias relativas se invierten. Asi, para el B3LYP, la energia relativa de la estructura IVm, la más estable, es de -6.01 $\mathrm{kcal} / \mathrm{mol}$, y la IIIm es de $-4.49 \mathrm{kcal} / \mathrm{mol}$, cuya diferencia relativa es de $1.50 \mathrm{kcal} / \mathrm{mol}$. En UMP2 para la estructura $\mathrm{IVm}$ exhibe una estabilidad relativa de $-1.08 \mathrm{kcal} / \mathrm{mol}$ y la estructura IIIm, la más estable, de $-1.62 \mathrm{kcal} / \mathrm{mol}$, con una diferencia de $0.50 \mathrm{kcal} / \mathrm{mol}$. En ambos casos, debido a que esta diferencia de energias es pequeña, a temperatura ambiente ambas conformaciones podrian ocurrir.

Para tratar de verificar cual de las estructuras de los radicales peroxilo en la adición en orto es la más estable, y validar esa fuerte interacción del oxígeno terminal del radical peroxilo con el hidrógeno del grupo $\mathrm{OH}$, se realizaron cálculos del tipo "single point" a nivel Coupled Cluster, con una mejor base, la $6-31 \mathrm{G}^{\star *}$, que adiciona funciones de polarización a los átomos de hidrógeno. Estos cálculos se efectuaron para la geometria de minima energia obtenida con B3LYP/6-31G* y UMP2/6-31G*, para las estructuras IIIm y IVm. Las energias a nivel CCD/6-31G**//B3LYP para las estructuras IIIm y IVm son 496.264853 y -496.264168 hartrees, mientras que a nivel $C C D / 6-31 G^{* *} / / U M P 2$ son 496.265562 y -496.264857 hartrees, respectivamente. Como se puede observar, la energia Coupled Cluster más baja corresponde efectivamente, a la adición en $\mathrm{Cl}$, con la geometria a nivel UMP2 $/ 6-31 \mathrm{G}^{*}$, lo cual nos indica que la estructura IIIm es la que más se favorece energéticamente.

Para los radicales peroxilo del aducto del carbono uno, Tabla 4.7 , se observa que las diferencias de energias relativas a nivel B3LYP/6-31G* y UMP2/6-31G*, presentan la 
misma tendencia, favoreciendo la adición en la posición 3. La estructura correspondiente al radical XIVm es la menos favorecida, ya que a nivel UMP2 tiene una energia de formaciōn muy endotérmica de $+38.55 \mathrm{kcal} / \mathrm{mol}$ y con B3LYP esta estructura no fue posible obtenerla. La adición en 2, cuya estructura corresponde a XIIIm y la adición en 4 , cuya estructura es XVm, son las más favorecidas energéticamente.

Cabe señalar que, a nivel UMP2 $/ 6-31 \mathrm{G}^{*}$, la diferencia de energía para las estructuras de los radicales peroxilo es positiva, mientras que a nivel B3LYP es negativa. En ambos métodos, la adición en 4 es la más favorecida, no obstante que presenta dos dobles enlaces no conjugados. La diferencias de energias relativas, entre las estructuras mâs favorecidas XIIIm y XVm, es de $1.5 \mathrm{kcal} / \mathrm{mol}$, lo que indica que a temperatura ambiente ambas estructuras son posibles.

\subsubsection{Radicales biciclo}

Como se observa en la Tabla 4.7, la mayoria de las estructuras de los radicales biciclos presentan energias de reacción muy endotérmicas, excepto para las estructuras VIm y VIo que corresponden a biciclos estables. La diferencia de energia para las estructuras de los radicales biciclos más estables es de $2.5 \mathrm{kcal} / \mathrm{mol}$ para B3LYP y de 2.9 $\mathrm{kcal} / \mathrm{mol}$ para UMP2. Esta estabilidad se debe, a que estas estructuras presentan un sistema alilico tipo $\pi$ deslocalizado. En efecto, en ésta estructura la densidad de espin está deslocalizada a través de los átomos de carbono $\mathrm{C} 4, \mathrm{C} 5$ y $\mathrm{C} 6$ que forman el sistema alilico. En cambio las demás estructuras solo presentan un doble enlace localizado.

Un análisis de población de Mulliken sobre la densidad de espin a nivel UMP2 de la estructura VIm, indica que está distribuida entre los carbonos C4, C5 y C6 con valores de $+0.67,-0.27$ y +0.67 respectivamente, equivalente al obtenido con B3LYP cuyos valores son, $+0.64,-0.24 \mathrm{y}+0.64$. Esto permite establecer que electrón desapareado del radical biciclo, se encuentra deslocalizado a travês de los átomos de carbono $\mathrm{C} 4, \mathrm{C} 5 \mathrm{y} \mathrm{C} 6$.

La estructura VIm es la más estable de todos los radicales biciclo (Figura 4.10a). Se puede obtener de manera directa a partir del radical peroxilo IIIm o del radical peroxilo IVm, a través de una ciclización de los dos oxigenos, en ambos métodos de cálculo el B3LYP y el UMP2.

Cabe señalar que lo mismo ocurre con la estructura Vlo, que representa al radical más estable cuando se adiciona el $\mathrm{O}_{2}$ del lado opuesto al radical $\mathrm{OH}$. Esta estructura 
también se puede obtener por la ciclización de los dos oxígenos del radical peroxilo IIIo o IVo, en ambos métodos de cálculo.

Con respecto a la adición en el carbono $\mathrm{C} 1$, y de acuerdo a la Tabla 4.8, se tiene que la estructura XVIm corresponde al radical biciclo más estable (Figura 4.10b). Esta estabilidad se atribuye a la estructura alilica tipo $\pi$ deslocalizado que presenta, tal como en el caso de la adición en orto. Es de hacer notar que este radical biciclo, de acuerdo a los resultados obtenidos, no se obtiene de manera directa de la ciclización del peroxilo más estable, que en este caso es el radical peroxilo XVm, sino del radical peroxilo XIIIm menos estable.

El análisis de población de Mulliken sobre la densidad de espin para la estructura XVIm, es muy parecida a la observada en la estructura VIm. Solo que en la estructura XVIm el electrón desapareado del radical biciclo, se encuentra deslocalizado a través de los átomos de carbono $\mathrm{C} 3, \mathrm{C} 4$ y $\mathrm{C} 5$.

Comparando las diferencias de energías relativas para los dos grupos de radicales biciclo, adición en orto y adición en $\mathrm{C} 1$, se puede establecer que debido a la diferencia tan pequeña entre XVIm y VIm de $0.14 \mathrm{kcal} / \mathrm{mol}$ con el método B3LYP, y de $1.36 \mathrm{kcal} / \mathrm{mol}$ con UMP2, a temperatura ambiente todas estas estructuras se podrian formar fácilmente y es factible que dichas estructuras sean intermediarios importantes en la oxidación del tolueno a nivel troposférico.

\subsubsection{Radicales tipo epóxido}

Para ambas adiciones, en orto y en $\mathrm{C} 1$, se obtuvieron en total siete estructuras tipo epóxido muy estables, con energias del orden de $30 \mathrm{kcal} / \mathrm{mol}$ por debajo de las estructuras de los radicales peroxilo y de $12 \mathrm{kcal} / \mathrm{mol}$ por debajo de las estructuras de los radicales biciclo.

Para la adición del $\mathrm{O}_{2}$ en posición orto, la estructura tipo epóxido que más se favorece energéticamente es la XIm (Figura 4.11a), la cual se obtiene a través de un relajamiento estructural que sufre la estructura VIm (que corresponde al radical peroxilo más estable), de manera directa, una vez que se rompe el enlace O-O. Esta estructura presenta un enlace tipo epóxido entre $\mathrm{C6}-\mathrm{O} 18-\mathrm{C} 1$ y un enlace tipo radical alcóxi entre el $\mathrm{C} 2-019$. Para el primero se tiene un enlace $\mathrm{C}-\mathrm{O}$ de $1.44 \AA$, una distancia C-C de $1.48 \AA$ y un ángulo $\mathrm{C}-\mathrm{O}-\mathrm{C}$ de $59.9^{\circ}$, y la distancia $\mathrm{C}-\mathrm{O}$ para radical alcóxi es de $1.38 \AA$. 
Un análisis de población de Mulliken para la estructura XIm muestra que el átomo de oxígeno del enlace epoxídico tiene una carga de -0.47 y el átomo de oxigeno del radical alcóxi tiene una carga de -0.30. La densidad electrónica de espín se localiza principalmente en el átomo de oxigeno del radical alcóxi con un valor de 0.92 , mientras que el átomo de oxigeno del enlace tipo epoxídico solo presenta una densidad electrónica de espin de 0.15 .

Con respecto a la adición en el carbono $\mathrm{C} 1$ se obtienen dos estructuras de minima energia. Con el método B3LYP/6-31G* se obtiene un radical tipo epóxido, cuya estructura es la XVIIIm (Figura $4.11 \mathrm{~b}$ ), con una diferencia de energia relativa de $-29.19 \mathrm{kca} / \mathrm{mol}$ comparable con la estructura XIm para la adición en orto. La estructura XIXm obtenida con B3LYP, presenta características similares a la estructura XIm, solo que aquí el enlace epoxídico se forma a través de $\mathrm{C6}-\mathrm{O} 18-\mathrm{C} 5$, con una distancia $\mathrm{C}-\mathrm{O}$ de $1.43 \AA$ y una distancia C-C de $1.48 \AA$, y el enlace tipo radical alcóxi se forma entre el C2O19, con una distancia C-O de $1.38 \AA$.

La estructura XIXm tanto a nivel B3LYP como a nivel UMP2 se puede obtener de manera directa de la estructura XVIm del radical peroxilo, una vez que se rompe el enlace $\mathrm{O}-\mathrm{O}$.

Un análisis de Mulliken para la estructura XIXm a nivel B3LYP/6-31G*, muestra que el oxígeno del enlace epoxidico tiene una carga de -0.43 y el oxígeno del radical alcóxi tiene una carga de -0.33 , mientras que la densidad electrónica de espin se encuentra localizada casi completamente en el oxigeno del radical alcóxi, con 0.86 , y en mucho menor proporción en el oxigeno del enlace epoxidico con 0.02. Esta estructura presenta un doble enlace localizado entre los carbonos $\mathrm{C} 2$ y $\mathrm{C} 3$.

Con el método UMP2/6-31G* se obtiene la estructura XIXm correspondiente a1 radical epóxido más estable de todas las estructuras, estudiadas en este trabajo, con una diferencia de energia relativa de $-34.90 \mathrm{kcal} / \mathrm{mol}$, casi $3 \mathrm{kcal} / \mathrm{mol}$ más baja que la estructura XIm. La estructura XIXm, a nivel UMP2 presenta dos enlaces epoxídicos formados a través del enlace $\mathrm{C} 6-\mathrm{O} 18-\mathrm{C} 5$ y del C2-O19-C3. Esta estructura es muy simétrica, ver Figura 4.11c. La distancia C-O es de $1.44 \AA$ y la distancia C-C es de $1.48 \AA$ $\mathrm{y}$ el ángulo de enlace $\mathrm{C}-\mathrm{O}-\mathrm{C}$ es de $59.9^{\circ}$. Las distancias $\mathrm{H} 17-\mathrm{O} 18$ y H17-O19 son equidistantes, igual a $2.51 \AA$, lo que hace que ésta estructura sea muy simétrica. 
Un análisis de Mulliken de la estructura XVIIIm a nivel UMP2/6-31G* muestra que los dos átomos de oxígeno presentan una carga de -0.46 y que la densidad electrónica de espin está localizada exclusivamente en el átomo de carbono C4. Esta estructura no presenta ningún doble enlace. 


\section{CONCLUSIONES}

En este trabajo, se han utilizado cálculos de orbitales moleculares para determinar las estructuras de minima energia de los posibles intermediarios y las superficies de energia potencial de las reacciones de oxidación de parafinas e hidrocarburos aromáticos (tolueno).

Se reportan nuevos resultados energéticos y estructurales de los reactivos, estados de transición y productos de dos reacciones sucesivas que involucran procesos radicalmolécula y que son las reacciones iniciales en la oxidación troposférica de las parafinas: la abstracción de un átomo de hidrógeno por el radical $\mathrm{OH} \bullet$ :

$$
\mathrm{RH}+\mathrm{OH} \bullet \rightarrow \mathrm{R} \bullet+\mathrm{H}_{2} \mathrm{O}
$$

y la adición de una molécula de oxigeno al radical alquilo:

$$
\mathrm{R} \bullet+\mathrm{O}_{2} \rightarrow \mathrm{RO}_{2} \bullet
$$

Las reacciones de metano, etano, propano, butano, iso-butano y neo-pentano fueron estudiadas por los métodos B3LYP y MP2 utilizando una base de orbitales 6$31 \mathrm{G}^{\star \star}$. Se analizaron en particular las diferencias en el comportamiento de las reacciones cuando se abstrae un hidrógeno primario, secundario o terciario. Se encuentran semejanzas muy evidentes en todos los casos en los que se trata de un mismo tipo de hidrógeno. De hecho, fue de gran ayuda en la optimización de las geometrias de las parafinas más grandes, el buscar los minimos absolutos por analogía con los del mismo tipo en parafinas menores. Los dos métodos dan resultados numéricos para las barreras y para las energias de reacción, que son muy diferentes de los resultados experimentales. Sin embargo, las tendencias son tan claras, y tan paralelas con ambos métodos, que se sugiere que se puede aplicar un factor de escala para corregir los valores calculados. Este escalamiento tomaria en cuenta el efecto túnel, muy importante en estas reacciones, asi como las deficiencias propias de cada método.

Los resultados escalados podrian entonces ser utilizados para descartar valores experimentales. Por ejemplo, de acuerdo a los valores experimentales, todos los radicales 
alquilos primarios reaccionan con oxigeno molecular con constantes de reacción de aproximadamente $7.5 \times 10^{-17} \mathrm{~cm}^{-3}$ molécula-1 $^{-1} \mathrm{~s}-1$, excepto el radical isobutilo, para el cual la constante es mucho menor. El valor de la energia de activación calculado en este trabajo, sin embargo, es parecido al de los demás, por lo que sugerimos que el valor experimental debe ser revisado.

En cuanto al estudio de las reacciones de oxidación del tolueno, se estudió la reacción de abstracción de hidrógeno de la cadena lateral por el radical $\mathrm{OH} \bullet$ :

$$
\mathrm{ArH}+\mathrm{OH} \bullet \rightarrow \mathrm{Ar} \bullet+\mathrm{H}_{2} \mathrm{O}
$$

Se encontró que, contrariamente a lo esperado, la barrera de reacción para la abstracción directa es del orden de la del metano, y mucho mayor a la reportada experimentalmente. Si bien no hemos encontrado un camino de reacción alternativo que explique la formación del benzaldehido, está claro que el mecanismo aceptado generalmente no puede ser correcto. Se continuará trabajando en este tema.

También se estudió la reacción de adición de $\mathrm{OH} \bullet$ al anillo aromático:

$$
\mathrm{ArH}+\mathrm{OH} \bullet \rightarrow \operatorname{ArH}(\mathrm{OH}) \bullet
$$

la cual puede ocurrir en diferentes posiciones. Tradicionalmente se consideraban únicamente la adición en las posiciones orto, meta y para, debido a que se han aislado los correspondientes productos de reacción, fenoles y nitro-toluenos. Sin embargo, hemos encontrado que la adición en la posición ipso, o sea, en el carbono substituido por el metilo, es también muy importante, $y$, de hecho, el método MP2 favorece el correspondiente aducto. Además, la barrera energética para su formación es también relativamente baja. Aún asumiendo que el método MP2 exagera la importancia relativa de este aducto, está claro que no debe despreciarse en un mecanismo completo de oxidación del tolueno. Falta encontrar cuál es el proceso por el cual este aducto desaparece, y a qué productos dá lugar. En este trabajo se han propuesto algunos esquemas, Figuras 4.3, 4.4 y 4.5 del Capitulo 4.

Los aductos $\mathrm{OH}$-tolueno reaccionan con oxigeno molecular para formar peroxilos:

$$
\operatorname{ArH}(\mathrm{OH}) \bullet+\mathrm{O}_{2} \rightarrow \operatorname{ArH}(\mathrm{OH}) \mathrm{O}_{2} \bullet
$$


los cuales pueden a continuación adquirir una variedad de estructuras isoméricas. En este trabajo se han estudiado más de veinte de estas estructuras, incluyendo, por primera vez, las que provienen del aducto en la posición ipso. Además, se pueden considerar dos conjuntos de isómeros diferentes, de acuerdo a si el radical OH• y el O-O están del mismo lado del anillo o de lados opuestos. En principio pueden ocurrir ambos tipos de estructuras, si bien la migración de uno a otro no es posible.

En este trabajo, encontramos que las estructuras más estables son los radicales epóxidos, que tienen energias más de $10 \mathrm{kcal} / \mathrm{mol}$ por debajo de las pocas estructuras biciclicas estables. De hecho, para que éstas sean estables, el puente O-O a través del anillo debe permitir que se forme un enlace C-C-C- tipo alilo en el anillo. Los radicales $\mathrm{O}_{2}-\mathrm{OH}$-tolueno menos estables son los peroxilos. Sus energías están aproximadamente $10 \mathrm{kcal} / \mathrm{mol}$ por encima de las de los biciclos estables, y de hecho, sus energias de reacción con respecto a los reactivos son de unas pocas $\mathrm{kcal} / \mathrm{mol}$. Los resultados obtenidos indican que solo dos de ellos se forman en reacciones exotermicas.

Se han encontrado dos estructuras de epóxidos particularmente muy estables, que corresponde a las estructuras XIs y XIXs. Sus energias a nivel B3LYP son muy similares, pero a nivel MP2 la última estructura se favorece por más de $3 \mathrm{kcal} / \mathrm{mol}$. Aunque, cabe señalar que esta estructura no se forma directamente del radical peroxilo más estable (estructura XVs), sino del radical peroxilo XIIIs menos favoprecido, a través del siguiente mecanismo:

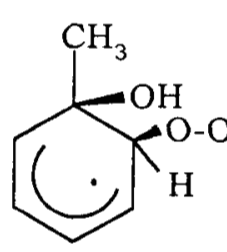

XIII
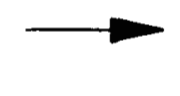

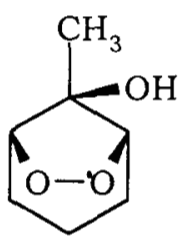

XVI

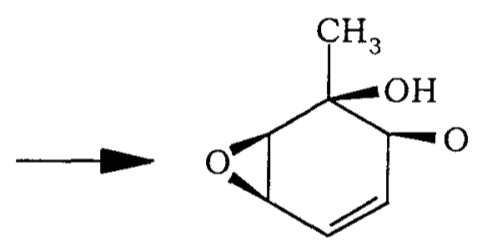

XIX

La adición de $\mathrm{O}_{2}$ al aducto orto favorece la formación del radical epóxido XIs que también es muy estable, a través del siguiente mecanismo de reacción: 
<smiles>CC1=CC=C[C@H](O)[C@@H]1OO</smiles>

IV
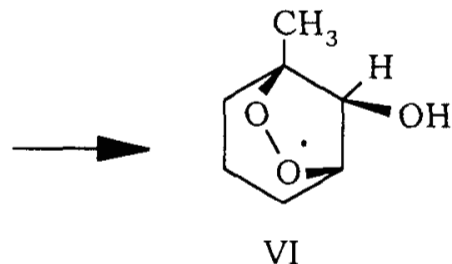

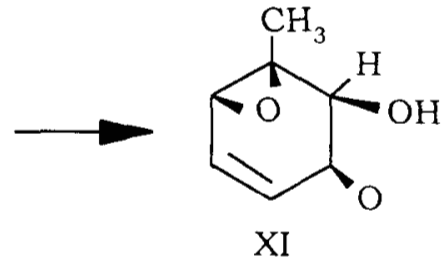

$\mathrm{XI}$

Aunque los resultados energéticos de los dos métodos teóricos empleados en este trabajo, no concuerdan exactamente, las tendencias observadas son muy parecidas, lo cual es muy significativo debido a que ambos métodos se basan en diferentes aproximaciones. Por otra parte, no hay datos experimentales sobre los radicales intermediarios que nos permita hacer una comparación. Recientemente $\mathrm{Yu}$ et al. [121-123], a través de espectrometria de masas encontraron evidencia experimental, sobre los intermediarios epóxido.

Para finalizar podemos establecer que, los resultados numéricos que presentamos en este trabajo pueden generar bases teóricas para la elucidación de un adecuado mecanismo de foto-oxidación de hidrocarburos aromáticos en la tropósfera. 


\section{ANEXO 1}

\section{MÉTODOS TEORICOS UTILIZADOS EN LOS CÁLCULOS}

En este anexo se describen los métodos teóricos que se emplearon en este trabajo.

\section{A.1. Teoria de perturbaciones de Møller-Plesset}

La teoria de perturbaciones de Møller-Plesset de segundo orden (MP2) es una forma de la teoria de perturbaciones Rayleigh-Schrödinger adaptada especialmente para tomar en cuenta los efectos de correlación en la aproximación Hartree-Fock [69-70]. El Hamiltoniano total del sistema de $\mathrm{N}$-electrones es:

$$
\mathrm{H}=\mathrm{H}_{0}+V
$$

donde el Hamiltoniano de orden cero $\mathrm{H}_{0}$ es el Hamiltoniano Hartree-Fock. $\mathrm{H}_{0}$ es la suma de los operadores monoelectrónicos de Fock $f(i)$ sobre los $\mathrm{N}$-electrones,

$$
\mathrm{H}_{0}=\sum_{i=1}^{N} f(i)=\sum_{i=1}^{N}\left\{h(i)+\sum_{j=1}^{N}\left[J_{j}(i)-K_{j}(i)\right]\right\}
$$

y $V$ representa la perturbación,

$$
V=\mathrm{H}-\mathrm{H}_{0}=\sum_{i<j}^{N} r_{i j}^{-1}-\sum_{i=1}^{N} \sum_{j=1}^{N}\left[J_{j}(i)-K_{j}(i)\right]
$$

En estas relaciones $h(i), J_{j}(i)$ y $K_{j}(i)$ son operadores monoelectrónicos. $\mathrm{El}$ primero es la suma de la energia cinética del i-ésimo electrón más la atracción del núcleo sobre dicho electrón y la repulsión entre núcleos. El segundo es el operador de coulomb, y el tercero el operador de intercambio. 
Para el nivel fundamental, la autofunción del problema de orden cero es la función de onda Hartree-Fock cuyo autovalor es la suma de las energías de los orbitales ocupados, i. e.,

$$
\left.\mathrm{H}_{0}\left|\Psi_{0}^{(0)}\right\rangle=\mathrm{E}_{0}^{(0) !} \Psi_{0}^{(0)}\right\rangle, \mathrm{E}_{0}^{(0)}=\sum_{i=1}^{N} \varepsilon_{i}
$$

Según la teoria de perturbaciones de Rayleigh-Schrödinger, la corrección de primer orden, a la energía del nivel fundamental es:

$$
E_{0}^{(1)}=\left\langle\Psi_{0}|V| \Psi_{0}\right\rangle=\left\langle\Psi_{0} \sum_{i<j}^{N} r_{i j}^{-1} \Psi_{0}-\Psi_{0} \sum_{i, j}^{N}\left(J_{j}(i)-K_{j}(i)\right) \Psi_{0}=-\frac{1}{2} \sum_{i, j}^{N}\left(J_{i j}-K_{i j}\right)\right.
$$

Por otra parte, se puede demostrar fácilmente que la energia Hartree-Fock es la suma de las correcciones de cero y primer orden:

$$
\begin{aligned}
\mathrm{E}_{H F} & =\sum_{i=1}^{N} h_{i}+\sum_{i<j}^{N}\left(J_{i j}-K_{i j}\right)=\sum_{i}^{N} h_{i}+\frac{1}{2} \sum_{i, j}^{N}\left(J_{i j}-K_{i j}\right) \\
& =\sum_{i=1}^{N} h_{i}+\sum_{i, j}^{N}\left(J_{i j}-K_{i j}\right)-\frac{1}{2} \sum_{i, j}^{N}\left(J_{i j}-K_{i j}\right)=\sum_{i=1}^{N} f_{i}-\frac{1}{2} \sum_{i, j}^{N}\left(J_{i j}-K_{i j}\right) \\
& =\sum_{i=1}^{N} \varepsilon_{i}-\frac{1}{2} \sum_{i, j}^{N}\left(J_{i j}-K_{i j}\right)=\mathrm{E}_{0}^{(0)}+\mathrm{E}_{0}^{(1)}
\end{aligned}
$$

Esto significa que, hasta primer orden, no hay efectos de correlación en la energia. Estos aparecen en las correcciones de segundo orden o a órdenes mayores.

Con base en la teoria de perturbaciones de Rayleigh-Schrödinger, la corrección de segundo orden a la energía se obtiene por,

$$
\mathrm{E}_{0}^{(2)}=\sum_{n} \frac{\left(\Psi_{0}|V| \Psi_{n}\right\rangle^{2}}{\mathrm{E}_{0}^{(0)}-\mathrm{E}_{n}^{(0)}}
$$


donde $E_{n}^{(0)}$ es la energía de la función de un estado excitado $\Psi_{n}^{(0)}$ del sistema. En la teoria de perturbaciones de Møller-Plesset las funciones $\Psi_{n}^{(0)}$ son determinantes formados por excitaciones a partir de estado basal de Hartree-Fock $\Psi_{0}^{(0)}$ sustituyendo espin orbitales ocupados por espin orbitales virtuales.

Porque $V$ solo contiene operadores monoelectrónicos y bielectrónicos, $\Psi_{n}$ no puede diferir de $\Psi_{0}$ en más de dos espin orbitales. Asi, se deben considerar las contribuciones a la correlación electrónica de excitaciones simples y dobles, las cuales, se calculan a continuación.

Para excitaciones simples, el estado excitado $\Psi_{a}^{r}$ difiere del $\Psi_{0}$ en un espín orbital (el espin orbital $\Phi_{a}$ de $\Psi_{0}$ se reemplaza por $\Phi_{r}$ en $\Psi_{a}^{r}$ ). Entonces el elemento de matriz es:

$$
\left\langle\Psi_{0}|V| \Psi_{a}^{r}\right\rangle=\left\langle\Psi_{0} \mathrm{H} \mid \Psi_{a}^{r}\right\rangle-\left\langle\Psi_{0} \mathrm{H}_{0} \Psi_{a}^{r}\right\rangle
$$

Escribiendo el determinante como el antisimetrizador $A$ aplicado a un producto de espin orbitales, $\Phi$, podemos escribir:

$$
\begin{aligned}
\left\langle\Psi_{0}|V| \Psi_{a}^{r}\right\rangle & =\left\langle A \Phi_{0}|\mathrm{H}| A \Phi_{a}^{r}\right\rangle-\left\langle A \Phi\left|\mathrm{H}_{0}\right| A \Phi_{a}^{r}\right\rangle \\
& =\left\langle\Phi_{0}\left|A^{+} \mathrm{H} A\right| \Phi_{a}^{r}\right\rangle-\left\langle\Phi_{0}\left|A^{+} \mathrm{H}_{0} A\right| \Phi_{a}^{r}\right\rangle \\
& =\left\langle\Phi_{0}|A \mathrm{H} A| \Phi_{a}^{r}\right\rangle-\left\langle\Phi_{0}\left|A^{+} \mathrm{H}_{0} A\right| \Phi_{a}^{r}\right\rangle,
\end{aligned}
$$

donde hemos utilizado el hecho de que el antisimetrizador $A$ es un operador hermitico, (ya que los operadores de permutación son unitarios).

Ahora bien, como las sumatorias en $\mathrm{H}$ y $\mathrm{H}_{0}$ son simétricas con respecto a las permutaciones de los electrones, ambos operadores conmutan con el antisimetrizador, i. e., 


$$
\left\langle\Psi_{0} V \mid \Psi_{a}^{r}\right\rangle=\left\langle\Phi_{0}\left|\mathrm{H} A^{2}\right| \Phi_{a}^{r}\right\rangle-\left\langle\Phi_{0}\left|\mathrm{H}_{0} A^{2}\right| \Phi_{a}^{r}\right\rangle
$$

$\mathrm{El}$ antisimetrizador es un operador casi idempotente:

$$
A=\frac{1}{\sqrt{N !}} \sum_{p}(-1)^{p} \hat{p}, \quad A^{2}=\sqrt{N !} A
$$

de modo que,

$$
\left\langle\Psi_{0} V \mid \Psi_{a}^{r}\right\rangle=\sum_{\wp}(-1)^{p}\left\langle\Phi_{0} \mathrm{H} \wp \Phi_{a}^{r}\right\rangle-\sum(-)^{p}\left\langle\Phi_{0} \mathrm{H}_{0} \wp \Phi_{a}^{r}\right\rangle
$$

Ahora tenemos que:

$$
\begin{aligned}
\sum_{\wp}(-1)^{p}\left\langle\Phi|\mathrm{H}| \wp \Phi_{a}^{r}\right\rangle & =h_{a r}+\sum_{I \neq a}^{N}\left\langle\operatorname{Ia}_{12}^{-1} I r\right\rangle-\left\langle\operatorname{Ia} r_{12}^{-1} r I\right\rangle \\
& =h_{a r}+\sum_{I \neq a}^{N}\left(J_{I}\right)_{a r}-\left(K_{I}\right)_{a r} \\
& =h_{a r}+\sum_{I}^{N}\left(J_{I}-K_{I}\right)_{a r} \\
& =\left[h+\sum_{I=0}^{N}\left(J_{I}-K_{I}\right)\right] \\
& =f_{a r}=\varepsilon_{r} \delta_{a r}
\end{aligned}
$$

y para la suma de los operadores monoelectrónicos de Fock,

$$
\sum_{\wp}(-1)^{p}\left\langle\Phi \mid \mathrm{H}_{0} \wp \Phi_{a r}\right\rangle=f_{a r}=\varepsilon_{r} \delta_{a r}
$$

Por lo tanto las excitaciones simples no contribuyen a la corrección de segundo orden a la energia. Este resultado es consecuencia del teorema de Brillouin. 
Siguiendo un procedimiento análogo al anterior para las excitaciones dobles $\Psi_{a h}^{r s}$, podemos evaluar los correspondientes elementos de matriz de $V$ :

$$
\left\langle\Psi_{0} V \mid \Psi_{a b}^{r s}\right\rangle=\sum_{\wp}(-1)^{p}\left\langle\Phi_{0} \sum_{i<j} r_{i j}^{-1} \wp \Phi_{a b}^{r s}-\sum_{\wp}(-)^{p} \Phi_{0} \sum_{i}\left[\sum_{j} J_{j}(i)-K_{j}(i)\right] \wp \Phi_{a b}^{r s}\right.
$$

El término bielectrónico es:

$$
\sum_{\wp}(-1)^{p}\left\langle\Phi_{0} \sum_{i<j} r_{i j}^{-1} \wp \Phi_{a b}^{r s}\right\rangle=\left\langle a b r_{12}^{-1} r s\right\rangle-\left\langle a b \mid r_{12}^{-1} s r\right\rangle
$$

mientras que el término monoelectrónico es cero:

$$
\sum_{\wp}(-1)^{p}\left\langle\Phi_{0} \sum_{i}\left[\sum_{j} J_{j}(i)-K_{j}(i)\right] \wp \Phi_{a b}^{r s}=0\right.
$$

En consecuencia, se puede escribir

$$
\left\langle\Psi_{0} V \mid \Psi_{a b}^{r s}\right\rangle=\left\langle a b r_{12}^{-1}\left(J-\wp_{12}\right) r s\right\rangle
$$

y sumando sobre todos los distintos pares de espin orbitales ocupados y virtuales, se obtiene:

$$
\mathrm{E}_{0}^{(2)}=\sum_{a<b, r<s} \frac{\left(a b_{12}-1\left(J-\wp_{12}\right) r s\right)^{2}}{\varepsilon_{a}+\varepsilon_{b}-\varepsilon_{r}-\varepsilon_{s}}
$$

Asi, la teoria de perturbaciones de Møller-Plesset MP2 incluye los efectos de correlación originados solamente de excitaciones dobles.

Las expresiones para las correcciones $\mathrm{E}_{0}^{(3)}$ y $\mathrm{E}_{0}^{(4)}$ son más complicadas y están incluidas en los métodos MP3 y MP4 respectivamente. 


\section{A.2 Contaminación de espin}

Para entender el problema de la contaminación de espin, es útil calcular el valor esperado de $S^{2}$ con respecto a un determinante de Slater. Para ello, se puede escribir la forma simétrica bien conocida de $S^{2}$ en términos de los operadores escalera de momento angular de espin:

$$
S^{2}=S_{-} S_{+}+S_{z}\left(S_{z}+1\right)
$$

donde para un sistema de $\mathrm{N}$-electrones, los operadores $S_{\alpha}$ son operadores aditivos sobre todos los electrones:

$$
S_{\alpha}=\sum_{\mu=1}^{N} S_{\alpha}(\mu)
$$

$\operatorname{con} \alpha=+, z,-$

En el método de Hartree-Fock, el estado del sistema se describe de manera aproximada a través de un determinante de Slater normalizado,

$$
\begin{aligned}
& D(1,2, \ldots, N)=\mid \Phi_{1}(1) \Phi_{2}(2) \ldots \Phi_{N}(N) \\
& =A \prod_{\mu=1}^{N} \Phi_{\mu}(\mu)=\frac{1}{\sqrt{N !}} \sum(-1)^{p} \wp \prod_{\mu=1}^{N} \Phi_{\mu}(\mu)
\end{aligned}
$$

donde el antisimetrizador $A$ se define como la suma de $N$ ! permutaciones de los $N$ electrones acomodados en un producto de espin orbitales. La suma sobre las permutaciones es antisimétrica debido a que cada permutación se asocia con el operador paridad y se multiplica por el factor $(-1)^{p}$.

Para evaluar el valor esperado de $S^{2}$ con respecto al estado D se hace uso del hecho de que los operadores de momento angular conmutan con los operadores de permutacion. Explícitamente, 
$\left[S_{\alpha}, \wp\right]=0$

Para el operador $\mathrm{S}_{\mathrm{z}}$ :

$$
\begin{aligned}
S_{z}|D\rangle & \left.=\sum_{\mu=1}^{N} S_{z}(\mu) A \prod_{v=1}^{N} \Phi_{v}(v)\right\rangle \\
& =\sum_{\mu=1}^{N} S_{z}(\mu) \frac{1}{\sqrt{N} !} \sum_{\wp}(-)^{p} \wp \prod_{v=1}^{N} \Phi_{v}(v) \\
& =\frac{1}{\sqrt{N} !} \sum_{\wp}(-)_{\wp}^{p} \sum_{\mu=1}^{N} S_{z}(\mu) \prod_{v=1}^{N} \Phi_{v}(v) \\
& =A \frac{1}{2}\left(n_{\alpha}-n_{\beta}\right) \prod_{v=1}^{N} \Phi_{v}(v) \\
& =\frac{1}{2}\left(n_{\alpha}-n_{\beta}\right) A\left|\prod_{v=1}^{N} \Phi_{v}(v)\right\rangle \\
\left.S_{z} D\right\rangle & \left.=\frac{1}{2}\left(n_{\alpha}-n_{\beta}\right) D\right\rangle,
\end{aligned}
$$

donde $n_{\alpha}$ y $n_{\beta}$ son el número de espín orbitales en $\mathrm{D}$ con espin alfa y espin beta, respectivamente. Con este resultado, se puede escribir inmediatamente la acción del último término en la ecuación (A.22) sobre el ket $|D\rangle$,

$$
S_{z}\left(S_{z}+1\right)|D\rangle=\frac{1}{2}\left(n_{\alpha}-n_{\beta}\right)\left[\frac{1}{2}\left(n_{\alpha}-n_{\beta}\right)+1\right]|D\rangle
$$

El primer término de la ecuación (A.19), se puede escribir como la suma de dos términos:

$$
S_{-} S_{+}=\sum_{\mu=1}^{N} \sum_{v=1}^{N} S_{-}(\mu) S_{+}(v)=\sum_{\mu=1}^{N} S_{-}(\mu) S_{+}(\mu)+\sum_{\mu \neq \nu}^{N} S_{-}(\mu) S_{+}(v)
$$


Cuando $\mu \mathrm{y} v$ son iguales, se tiene que:

$S_{-}(\mu) S_{+}(\mu) \alpha(\mu)=0, \quad S_{-}(\mu) S_{+}(\mu) \beta(\mu)=\beta(\mu)$

de modo que:

$\left.\sum_{\mu=1}^{N} S_{-}(\mu) S_{+}(\mu) D\right\rangle=n_{\beta} D$

El resultado que hemos obtenido hasta ahora, es:

$\left.S^{2}|D\rangle=\left\{\sum_{\mu=1}^{N} S_{-}(\mu) S^{+}(\mu)+n_{\beta}+\frac{1}{2}\left(n_{\alpha}-n_{\beta}\right)\left[\frac{1}{2}\left(n_{\alpha}-n_{\beta}\right)+1\right]\right\} D\right\rangle$

Para evaluar el elemento de matriz,

$$
\begin{aligned}
\left\langle D \mid \sum_{\mu \neq v}^{N} S_{-}(\mu) S_{+}(v) D\right\rangle & =A \prod_{i=1}^{N} \Phi_{i}(i) \sum_{\mu=v}^{N} S_{-}(\mu) S_{+}(v) A \prod_{j=1}^{N} \Phi_{j}(j) \\
& =\prod_{i=1}^{N} \Phi_{i}(i) A^{2} \sum_{i=1}^{N} S_{-}(\mu) S_{+}(v) \mid \prod_{j=1}^{N} \Phi_{j}(j) \\
& =\sqrt{N !}\left\langle\prod_{i=1}^{N} \Phi_{i}(i) A \sum_{\mu \neq v}^{N} S_{-}(\mu) S_{+}(v) \prod_{j=1}^{N} \Phi_{j}(j)\right.
\end{aligned}
$$

se utilizan las propiedades del antisimetrizador $A$ :

$$
A^{+}=A, \quad A^{2}=\sqrt{N !} A
$$

Escribiendo los espin orbitales como:

$$
\Phi_{i}(i)=\varphi_{i}^{w}(i) \omega(i)
$$


donde $\omega$ es una funciön de espin, $\alpha \circ \beta, y$ tomando en cuenta que

$$
S_{-}(\mu) S_{+}(v) \alpha(\mu) \beta(v)=\beta(\mu) \alpha(v),
$$

se tiene que:

$$
\begin{aligned}
& D \sum_{\mu \neq \nu}^{N} S_{-}(\mu) S_{+}(v) D \\
& =\sqrt{N !} \ldots \varphi_{\mu}^{\alpha}(\mu) \alpha(\mu) \ldots \varphi_{\nu}^{\beta}(v) \beta(v) \ldots A \sum_{\mu \neq v}^{N} S_{-}(\mu) S_{+}(v) \ldots \varphi_{\mu}^{\alpha}(\mu) \alpha(\mu) \ldots \varphi_{v}^{\beta}(v) \beta(v) \ldots \\
& =\sqrt{N !} \sum_{\mu \neq \nu}^{N} \ldots \varphi_{\mu}^{\alpha}(\mu) \alpha(\mu) \ldots \varphi_{\nu}^{\beta}(v) \beta(v) \ldots A \ldots \varphi_{\mu}^{\alpha}(\mu) \beta(\mu) \ldots \varphi_{v}^{\beta}(v)_{\alpha}(v) \ldots \\
& =\sum_{\mu \neq \nu}^{N}\left(\ldots \varphi_{\mu}^{\alpha}(\mu) \alpha(\mu) \ldots \varphi_{v}^{\beta}(v) \beta(v) \ldots I-\wp_{\mu \nu} \ldots \varphi_{\mu}^{\alpha}(\mu) \beta(\mu) \ldots \varphi_{v}^{\beta}(v) \alpha(v) \ldots\right. \\
& =-\sum_{\mu \neq v}^{N}\left(\ldots \varphi_{\mu}^{\alpha}(\mu) \alpha(\mu) \ldots \varphi_{v}^{\beta}(v) \beta(v) \ldots \wp_{\mu v} \ldots \varphi_{\mu}^{\alpha}(\mu) \beta(\mu) \ldots \varphi_{\nu}^{\beta}(v) \alpha(v) \ldots\right) \\
& =-\sum_{\mu \neq v}^{N}\left(\ldots \varphi_{\mu}^{\alpha}(\mu) \alpha(\mu) \ldots \varphi_{v}^{\beta}(v) \beta(v) \ldots \ldots \varphi_{v}^{\beta}(\mu) \alpha(\mu) \ldots \varphi_{\mu}^{\alpha}(v) \beta(v) \ldots\right)
\end{aligned}
$$

Debido a la ortogonalidad entre las funciones de espin, los únicos términos diferentes de cero son aquellos en los cuales cada electrón tiene el mismo espín en ambos miembros. Además, el conjunto de orbitales de un espin determinado es ortonormal. Tenemos finalmente que:

$$
\begin{aligned}
\left(D \sum_{\mu \neq \nu}^{N} S_{-}(\mu) S_{+}(\nu) D\right) & \left.=-\sum_{\mu \neq \nu}^{N}\left\langle\varphi_{\mu}^{\alpha} \varphi_{\nu}^{\beta}\right\rangle \varphi_{\nu}^{\beta} \varphi_{\mu}^{\alpha}\right\rangle \\
& =-\sum_{\mu \neq \nu}^{N}\left\langle\varphi_{\mu}^{\alpha} \mid \varphi_{\nu}^{\beta}\right\rangle^{2}
\end{aligned}
$$




$$
S^{2}|D\rangle=\left\{-\sum_{\mu \neq \nu}^{N}\left|\left\langle\varphi_{\mu}^{\alpha} \mid \varphi_{v}^{\beta}\right\rangle\right|^{2}+n_{\beta}+\frac{1}{2}\left(n_{\alpha}-n_{\beta}\right)\left[\frac{1}{2}\left(n_{\alpha}-n_{\beta}\right)+1\right]\right\}|D\rangle
$$

Este resultado general se obtiene cuando D se construye como un producto de espin orbitales no restringido.

En el caso particular en el que $n_{\beta}$ orbitales están doblemente ocupados, la sumatoria de la ecuación (A.33) se escribe como:

$$
\left\langle D \mid \sum_{\mu \neq \nu}^{N} S_{-}(\mu) S_{+}(v) D\right\rangle=-\sum_{\mu=1}^{n_{\beta}}\left\langle\varphi_{\mu}^{\alpha} \mid \varphi_{\mu}^{\beta}\right\rangle\left\langle\varphi_{\mu}^{\beta} \mid \varphi_{\mu}^{\alpha}\right\rangle
$$

y como $\varphi_{\mu}^{\alpha}=\varphi_{\mu}^{\beta}$, tenemos:

$$
\left\langle D \mid \sum_{\mid \mu \neq \nu}^{N} S_{-}(\mu) S_{+}(v) D\right\rangle=-\sum_{\mu=1}^{n_{\beta}}(1)=-n_{\beta}
$$

La ecuación resultante final es:

$$
S^{2}|D\rangle=\left\{\frac{1}{2}\left(n_{\alpha}-n_{\beta}\right)\left[\frac{1}{2}\left(n_{\alpha}-n_{\beta}\right)+1\right]\right\}|D\rangle
$$

que corresponde a un estado de espin puro.

En un cálculo Hartree-Fock no restringido los electrones alfa ocupan orbitales diferentes de los que ocupan los electrones beta, y, en general, los elementos $\left\langle\Phi_{\mu}^{\alpha} \mid \Phi_{\nu}^{\beta}\right\rangle$ en la ecuación (A.34) son menores que uno de modo que la suma de la ecuación (A.36) es menor que $n_{\beta}$. Por lo tanto, el resultado de operar con $\mathrm{S}^{2}$ sobre un determinante de Slater es mayor que $S(S+1)|D\rangle$, y se dice que hay contaminación de espin. En el caso particular de la función UHF de un estado doblete, por ejemplo, en vez de 0.75 se obtiene un valor un poco mayor, que puede ir desde 0.75 hasta casi 2.0 . 


\section{A.3 Eliminación de la contaminación de espin}

Una manera de representar la función de onda no restringida en términos de funciones de espin puro es escribir:

$$
\Psi_{U H F}=c_{1} \Psi_{\text {doblete }}+c_{2} \Psi_{\text {cuartete }}+c_{3} \Psi_{\text {sextete }}+\ldots
$$

En general la máxima contaminación de un estado de espin $\mathrm{S}$ proviene de la función de espin $S+1$. Si $\Psi_{0}$ es la función UHF, el operador de aniquilación

$$
\hat{A}_{S+1}=\frac{\hat{S}^{2}-(s+1)(s+2)}{\left\langle\Psi_{0} \hat{S}^{2} \mid \Psi_{0}\right\rangle-(s+1)(s+2)}
$$

actuando sobre $\Psi_{0}$ permite eliminar la contaminación correspondiente a $\mathrm{S}+1$, y se obtiene una función de onda de Hartree-Fock no restringido proyectada, $\Psi_{P U H F}$ :

$$
\Psi_{P U H F}=\hat{A}_{S+1} \Psi_{0}=\Psi_{0}+\frac{\sum_{i \neq 0} \Psi_{j}\left\langle\Psi_{j \mid}\left|\hat{S^{2}}\right| \Psi_{0}\right\rangle}{\left(\Psi_{0}\left|\hat{S}^{2}\right| \Psi_{0}\right\rangle-(s+1)(s+2)}=\Psi_{0}+\Psi_{1}
$$

con un valor esperado de $\hat{S}^{2}$ más cercano a $S(S+1)$.

En la ecuación anterior el denominador de $\hat{A}_{S+1}$ se encarga de que la función $\Psi_{P U H F}$ esté normalizada y las $\Psi_{j}$ son determinantes correspondientes a excitaciones del determinante $\Psi_{0}$ obtenidos utilizando los orbitales virtuales UHF. La suma sobre las $\Psi_{j}$ puede ser restringida a las excitaciones dobles, debido a que $\hat{\mathrm{H}}$ contiene solamente operadores monoelectrónicos y bielectrónicos y a que la función de onda UHF satisface el 
teorema de Brillouin, que establece que los determinantes monoexcitados $\left|\Psi_{a}^{r}\right\rangle$ no interactúan con el determinante UHF, $\left|\Psi_{0}\right\rangle$ :

$$
\left\langle\Psi_{0} \mid \mathrm{H} \Psi_{a}^{r}\right\rangle=0
$$

La fórmula para la energia de Hartree-Fock proyectada es:

$$
\begin{aligned}
\mathrm{E}_{P U H F} & =\left\langle\Psi_{0}|\hat{\mathrm{H}}| \Psi_{0}\right\rangle+\frac{\sum_{i \neq 0}\left\langle\Psi_{0}|\hat{\mathrm{H}}| \Psi_{i}\right\rangle\left\langle\Psi_{i}\left|\hat{A_{S+1}}\right| \Psi_{0}\right\rangle}{\left\langle\Psi_{0}\left|\hat{A_{S+1}}\right| \Psi_{0}\right\rangle} \\
& =\left\langle\Psi_{0}|\hat{\mathrm{H}}| \Psi_{0}\right\rangle+\frac{\sum_{i \neq 0}\left\langle\Psi_{0}|\hat{\mathrm{H}}| \Psi_{i}\right\rangle\left\langle\Psi_{i} \mid \hat{S}^{2} \Psi_{0}\right\rangle}{\left\langle\Psi_{0}\left|\hat{S^{2}}\right| \Psi_{0}\right\rangle-(s+1)(s+2)} \\
& =E_{U H F}+\Delta \mathrm{E}_{P U H F}
\end{aligned}
$$

Es importante hacer notar que en esta expresión la proyección ha sido aplicada después de realizar el cálculo autoconsistente. La justificación para utilizar las energías proyectadas es totalmente empirica. Sin embargo, muchos autores coinciden en que los resultados que se obtienen para las energias reproducen los resultados experimentales mejor que las energias no proyectadas, sobre todo en el cálculo de barreras de reacción

Sosa y Schlegel [71-74] han desarrollado expresiones para la proyección de espín para energias UMPn. Estas expresiones se encuentran integradas en el programa Gaussian.

\section{A.4 Teoria de funcionales de la densidad (DFT)}

Con base en el teorema de Hohenberg y Kohn [75], la energia total de un sistema se puede describir como un funcional de la densidad de carga. DFT utiliza esta definición de energia como punto de partida y los refinamientos que le siguen se relacionan con el 
mejoramiento del funcional [76]. Los cálculos con DFT se realizan con método iterativo de auto consistencia de Kohn y Sham [77.

En DFT, la energia se expresa como:

$$
\mathrm{E}=\mathrm{E}_{k}[\rho]+\mathrm{E}_{c}[\rho]+\mathrm{E}_{x c}[\rho]
$$

donde $\mathrm{E}_{k}, \mathrm{E}_{c}, \mathrm{y} \mathrm{E}_{x c}$, representan las contribuciones cinéticas, coulómbicas $\mathrm{y}$ de intercambio-correlación, respectivamente, a la energia y $\rho$ representa- la densidad de carga. Es un teorema fundamental de DFT que la energia se pueda expresar como un funcional de $\rho$. Los primeros dos términos tienen análogos en la teoria clásica, mientras que $\mathrm{E}_{x c}$ surge de efectos exclusivamente mecánico cuánticos.

Para sistemas de capas cerradas, la densidad se representa por una expansión de orbitales moleculares, $\Phi_{i}$ :

$$
\rho(r)=2 \sum_{i}^{o c c} \Phi_{i}^{*}(r) \Phi_{i}(r)
$$

donde la multiplicación por 2 asume que los orbitales están cada uno doblemente ocupados. Estos orbitales moleculares se expanden en combinaciones lineales de orbitales centrados en átomos, $\chi_{\mu}$.

$$
\Phi_{i}(r)=\sum_{\mu} C_{\mu i} \chi_{\mu}(r)
$$

Los coeficientes $C_{\mu i}$ producen orbitales moleculares como combinaciones lineales de orbitales atómicos, y se les conoce como LCAO o simplemente como coeficientes OM (orbitales moleculares). Los orbitales atómicos $\chi_{\mu}$ pueden ser cualquier base razonable, tales como, funciones gaussianas, funciones de Slater, u ondas planas. Por ejemplo, el Gaussian 94 o 98, asî como algunos programas basados en DFT, como el deMon-KS [78], Dgauss [79], etc, utilizan LCAO de tipo gaussiano (GTO). Esta representación permite describir con bastante precisión la estructura electrónica de átomos y moléculas en donde los electrones forman enlaces quimicos localizados en las regiones internucleares. Otros programas tipo DFT utilizan LCAO de tipo Slater, tal como el código de Amsterdam, el 
programa ADF ${ }^{800}$. En sistemas en donde los electrones estân muy deslocalizados, como en el caso sistemas periodicos, es conveniente usar LCAO del tipo ondas planas. En este trabajo utilizaremos LCAO-GTO, en cálculos ab-initio, basados en la teoria de HartreeFock.

Se requiere que los orbitales sean ortonormales,

$$
\int \Phi_{i} \Phi_{j} d \tau=\delta_{i j}
$$

lo cual impone la siguiente restricción sobre los coeficientes de los $\mathrm{OM}$,

$$
C^{+} S C=1
$$

donde $S$ es la matriz de traslape:

$$
S_{\mu v}=\int \chi_{\mu} \chi_{v} d t
$$

En términos de orbitales atómicos, la energia cinética es,

$$
E_{k}=-\frac{1}{2} \sum_{\mu i} C_{\mu i} C_{v i}\left\lceil\chi_{\mu}(r) \nabla^{2} \chi_{\nu}(r) d t\right.
$$

La energía coulómbica se puede dividir en contribuciones de atracción electrónnucleo,

$$
E_{c}(1 e)=\sum_{i, A} \int \frac{\Phi_{i}(r) Z_{A} \Phi_{i}(r)}{r-R_{A}}
$$

repulsión electrón-electrón,

$$
E_{c}(2 e)=2 \sum \iint_{i j} \Phi_{i}\left(r_{i}\right) \Phi_{i}\left(r_{i}\right)\left(\frac{1}{r_{1}-r_{2}}\right) \Phi_{j}\left(r_{2}\right) \Phi_{j}\left(r_{2}\right) d t_{1} d t_{2}
$$

y repulsiôn núcleo-núcleo: 


$$
E_{n u c}=\sum_{A<B} \frac{Z_{A} Z_{B}}{R_{A}-R_{B}}
$$

El subindice A corre sobre todos los átomos en la molécula y los subindices, i, j, corren sobre todos los orbitales moleculares ocupados.

En la teoria de funcionales de la densidad, la energia de intercambio-correlación total se representa en términos de la densidad multiplicada por algún operador, $\varepsilon_{x c}$, el cual es un funcional de la densidad de carga:

$$
E_{x c}=\int \rho \varepsilon_{x c}[\rho] d \tau
$$

La relación siguiente:

$$
v_{x c}(r)=\delta E_{x c}[\rho(r)] / \delta \rho(r)
$$

define el potencial de intercambio y correlación a usar en las ecuaciones autoconsistentes en DFT y que son las siguientes ${ }^{[\mathbf{8} 1]}$ :

$$
\left(-\frac{1}{2} \nabla^{2}+V(r)+\int \frac{\rho(r)}{r-r \mid} d r+\frac{\delta E_{x c}}{\delta \rho}\right) \Phi_{i}=\varepsilon_{i} \Phi_{i}
$$

donde la densidad $\rho$ se obtiene de la ecuación (A.44).

Con base, en las definiciones anteriores podemos expresar la energia como,

$$
E=2 \sum_{i}^{o c c} h_{i i}+2 \sum_{i}^{o c c}(i i \mid j j)+2 \sum_{i}^{o c c}\left(i\left|\varepsilon_{x c}\right| i\right)+E_{n u c}
$$

Esta ecuación es para un sistema de capa cerrada, es decir cada orbital está doblemente ocupado. La extensión a sistemas de capa abierta se hace de manera directa.

El funcional se puede separar en contribuciones de intercambio y de correlación. Si solo se tiene la correlación de intercambio, entonces la teoria se reduce al método de Hartree-Fock-Slater [82]. La aproximación de densidad de espin local (LSDA) asume que 
$\varepsilon_{x c}$ es un funcional únicamente de la densidad de carga uniforme en un punto dado en el espacio, $\rho(r)$.

En la literatura han aparecido varios funcionales de este tipo, aunque el funcional más utilizado es el de Vosko, Wilk y Nusair (VWN). Por otra parte, es posible tomar en cuenta las inhomogeneidades en la densidad al formular un funcional que dependa de la densidad y del gradiente de la densidad en un punto del espacio, $\varepsilon_{x c}=\varepsilon_{x c}[\rho, \mid \nabla \rho]$. Estos funcionales se conocen como funcionales no-locales (NL) o corregidos por el gradiente, tal como el del gradiente generalizado o GGA, y existe un gran número de este tipo de funcionales en la literatura. Se ha encontrado que la LSDA determina con una exactitud razonable la geometria de las moléculas o cúmulos de átomos. Sin embargo, las propiedades energéticas tales como energias de unión, potenciales de ionización, etc, no son bien descritas por este tipo de funcionales. En los cálculos de estructura electrónica, la geometria obtenida con funcionales LSDA y NL son muy similares, siendo ligeramente mejor (al compararla con el experimento), la geometria NL. Los funcionales no-locales mejoran substancialmente el cálculo o estimación de las energias de unión. Entre los funcionales NL más ampliamente utilizados están el BLYP y el funcional hibrido B3LYP, que han mostrado que pueden dar resultados con precisión quimica en una gran variedad de sistemas atómicos o moleculares.

En particular, en este trabajo hemos utilizado el funcional B3LYP, el cual ha sido ampliamente aplicado en sistemas de radicales libres, en donde la geometria y las propiedades quimicas obtenidas con este funcional, concuerdan muy bien con los resultados experimentales [83-87].

\section{A.5 Aproximación Coupled Cluster (CCA) ${ }^{[69]}$.}

En este método, la energia de correlación se escribe como:

$$
\begin{aligned}
& \mathrm{E}_{c o r r}=\sum_{a<b} e_{a b} \\
& e_{a h}=\sum_{r<s} c_{a b}^{r s}\left\langle\Psi_{0}|\mathrm{H}| \Psi_{a b}^{r s}\right\rangle
\end{aligned}
$$


donde $e_{a b}$ es la energía de correlación que resulta de la interacción entre el par de electrones que ocupan los espin orbitales $\chi_{a}$ y $\chi_{b}$.

Los coeficientes $c_{a b}^{r s}$ son, en principio, los obtenidos en un cálculo de interacciones de configuraciones completo (full $\mathrm{CI}$ ), resolviendo la ecuación secular con una función de onda:

$$
\begin{aligned}
\left|\Phi_{0}\right\rangle= & c_{0}\left|\Psi_{0}\right\rangle+\sum_{a r} c_{a}^{r}\left|\Psi_{a}^{r}\right\rangle+\sum_{a<b, r<s} c_{a b}^{r s}\left|\Psi_{a b}^{r s}\right\rangle \\
& +\sum_{a<b<c, r<s<t} c_{a b c}^{r s t}\left|\Psi_{a b c}^{r s t}\right\rangle+\sum_{a<b<c<d, r<s<l<u} c_{a b c d}^{r s t u}\left|\Psi_{a b c d}^{r s t u}\right\rangle+\ldots
\end{aligned}
$$

En la aproximación de pares electrónicos independientes (IEPA), las energias de los pares $e_{a b}$ se obtienen a partir de una función de onda de interacción de configuraciones que contienen solamente excitaciones dobles de los orbitales del par electrónico $a b$. En la aproximación Coupled Cluster (CCA), el método anterior, IEPA, se extiende para incorporar el acoplamiento entre diferentes pares electrónicos $a b$ y $c d$. Pero en vez de utilizar los coeficientes $c_{a b c d}^{r s t u}$ de las excitaciones cuádruples del CI, éstos se aproximan como productos de los coeficientes de excitaciones dobles:

$$
c_{a b c d}^{r s t u} \cong c_{a b}^{r s} * c_{c d}^{n u}
$$

El simbolo * indica que en realidad son, 18 productos de coeficientes de excitaciones dobles.

En términos de éstos coeficientes, las ecuaciones básicas de la teoria de CCD son las siguientes:

$$
\left\langle\Psi_{a b}^{r s}|\mathrm{H}| \Psi_{0}\right\rangle+\sum_{c<d, t<u}\left\langle\Psi_{a b}^{r s}\left|\mathrm{H}-\mathrm{E}_{0}\right| \Psi_{c d}^{\prime u}\right\rangle c_{c d}^{i u}-\sum_{c<d, t<u}\left\langle\Psi_{0}|\mathrm{H}| \Psi_{c d}^{\prime u}\right\rangle\left\langle c_{a b}^{r s}{ }^{*} c_{c d}^{i u}\right\rangle=0
$$

Si se incluyen las excitaciones simples y triples, la teoria se denomina CCSD. 


\section{A.6 Superficies de energia potencial}

El curso de una reacción quimica se determina utilizando la función de energia potencial para el movimiento nuclear $E\left(q_{\alpha}\right)$, donde $q_{\alpha}$ indica las coordenadas de los $\mathrm{N}$ núcleos de las moléculas reactantes. Para una molécula diatómica, $E$ es una función de una variable, la distancia internuclear $R$, y $E(R)$ es la curva de energia potencial [88].

La energia $E\left(q_{a}\right)$ es una función de las $3 \mathrm{~N}-6$ variables internas que describen la geometría de la molécula. Se resta 6 al numero total de variables de una molécula de $N$ átomos, pues se separan los movimientos translacional y rotacional. En general, $E\left(q_{\alpha}\right)$ es una superficie en un espacio abstracto de $3 \mathrm{~N}-6$ dimensiones. Para encontrar $E\left(q_{\alpha}\right)$ se debe resolver la ecuación de Schrödinger de los electrones para un gran número de configuraciones nucleares.

El camino de mínima energia que conduce de los reactantes a los productos se conoce como coordenada de reacción. Para la reacción inversa, la superficie $E\left(q_{\alpha}\right)$ es la misma que para la reacción directa, pero el camino de reacción se recorre en sentido opuesto. El punto de máxima energia potencial en la coordenada de reacción es el estado de transición o complejo activado y se trata de un punto de ensilladura en la superficie $E\left(q_{\alpha}\right)$, ya que es un punto máximo sobre el camino de reacción de mínima energia. El estado de transición no corresponde a una molécula estable. La diferencia de energía entre el estado de transición y los reactantes es la barrera para la reacción directa.

En principio, cuando se conoce la superficie $E\left(q_{a}\right)$, es posible calcular la constante de velocidad de reacción $k$, en función de la temperatura. Además, hay una probabilidad significativa de que las molêculas atraviesen la superficie de reacción por caminos diferentes del de minima energia. En particular, hay que tener en cuenta el efecto túnel mecanico-cuántico a través de la barrera. La constante de velocidad depende no solo de la altura de la barrera, sino también de la forma global de la superficie de potencial para la reacción.

Para una discusión cualitativa, se hace uso del hecho de que una altura grande de la barrera implica una constante de velocidad pequeña y una barrera pequeña implica una reacción rápida. 
Para reacciones que tienen un comportamiento tipo Arrhenius, o sea para las cuales la constante de velocidad $\mathrm{k}$ varia con la temperatura de acuerdo a la relación $k=\mathrm{A}$ $\exp \left(-E_{a} / \mathrm{RT}\right)$, se puede determinar la energia de activación experimental $E_{a}$ a partir de la pendiente de una gráfica de log $k$ contra $1 / T$ [89-90].

\section{A.7 Caracterización del estado de transición}

La idea central de la teoria del estado de transición es que la altura de la barrera que separa los reactantes de los productos, se relaciona de manera directa con la velocidad de la reacción [88-89]. La posición del minimo en la coordenada de reacción describe directamente las geometrias de equilibrio de los reactantes y productos, la posición del máximo describe la estructura del estado de transición, que en el curso de la reacción no se puede medir, y finalmente las energias relativas de los reactantes y productos relacionan de manera directa la abundancia de esas especies a tiempo infinito.

El estado de transición, es decir el punto máximo en la coordenada de reacción o punto de ensilladura, sirve para caracterizar matemáticamente una coordenada de reacción. Los reactantes, los productos, y el estado de transición son puntos estacionarios en la superficie de energía de potencial. En una dimensión, el estado de transición es el punto donde la derivada total de la energía $E$ con respecto a la coordenada de reacción $R$, es cero,

$$
\frac{d E}{d R}=0
$$

y la segunda derivada de la energia es negativa,

$$
\frac{d^{2} E}{d R^{2}}<0
$$

Para una molécula de $\mathrm{N}$ átomos el sistema se describe mediante una matriz de las segundas derivadas parciales de la energia con respecto a $3 \mathrm{~N}-6$ variables, $q_{\alpha}$. En este caso, el hessiano se define como: 


$$
\text { Hessiano }=\left(\begin{array}{ccc}
\frac{\partial^{2} E}{\partial R_{1}^{2}} & \frac{\partial^{2} E}{\partial R_{1} \partial R_{2}} & \bullet \\
\frac{\partial^{2} E}{\partial R_{2} \partial R_{1}} & \frac{\partial^{2} E}{\partial R_{2}^{2}} & \\
\bullet & & \\
\bullet & & \frac{\partial^{2} E}{\partial R_{3 N-6}^{2}}
\end{array}\right)
$$

Existen $3 N-6$ coordenadas geométricas, que permiten diagonalizar esta matriz, es decir:

$$
\left(\begin{array}{ccc}
\frac{\partial^{2} E}{\partial \xi_{1}^{2}} & & 0 \\
& \frac{\partial^{2} E}{\partial \xi_{2}^{2}} & \\
0 & & \frac{\partial^{2} E}{\partial \xi_{3 N-6}^{2}}
\end{array}\right)
$$

donde las $\xi_{i}$ se denominan coordenadas normales.

En este sistema de coordenadas, un mínimo se caracteriza por todas las segundas derivadas parciales positivas,

$$
\frac{\partial^{2} E}{\partial \xi_{i}^{2}}>0
$$

donde $i=1,2, \ldots 3 \mathrm{~N}-6$.

Mientras que el estado de transición se caracteriza por tener todas las segundas derivadas parciales de la energia positivas, excepto una, que corresponde a la coordenada de reacción $\xi_{p}$, para la cual la derivada parcial es negativa,

$$
\frac{\partial^{2} E}{\partial \xi_{p}}<0
$$


La posición de los minimos a lo largo de la coordenada de reacción proporciona las estructuras de equilibrio de estructuras estables. Las energias relativas de los minimos generan información acerca de las estabilidades relativas de las estructuras estables. Las energias de los máximos relativos a los minimos entre los cuales se encuentran, caracterizan la facilidad o dificultad de interconversión de estructuras estables.

Las estructuras de minima energia se caracterizan por tener todas sus frecuencias de vibración positivas, mientras que las estructuras de los estados de transición presentan una frecuencia de vibración negativa (imaginaria), entre -400 y $-2000 \mathrm{~cm}^{-1}$. 


\section{ANEXO 2 METODOLOGÍA DE LOS CÁlCULOS}

\section{B.1 Optimización de estructuras}

La optimización de las estructuras se llevó a cabo con los modelos PM3, UHF, UMP2 y B3LYP, utilizando los programas Spartan [93], y Gaussian 94 [94]. Para la visualización de las estructuras, los orbitales moleculares, las densidades de espin, y las frecuencias de vibración, se utilizaron los programas Spartan y GaussView [95].

El estudio por orbitales moleculares, de la reacción de abstracción de hidrógeno de las parafinas por el radical $\mathrm{OH} \bullet$, desde el metano hasta neo-pentano, asi como la adición de $\mathrm{O}_{2}$ al radical alquílico correspondiente, $\mathrm{R} \bullet$, se inició optimizando completamente las estructuras con el método semiempírico PM3 y posteriormente se refinó el cálculo con métodos ab-initio en la opción de Hartree-Fock no restringido (UHF), y con el método UMP2 con la base $6-31 \mathrm{G}^{\star *}$. Posteriormente, tomando la geometria optimizada con UMP2/6-31G** se realizaron cálculos "single point" con la teoría de funcionales de la densidad, utilizando el funcional de gradiente generalizado B3LYP.

Debido a que en la reacción de abstracción, hay una transferencia de hidrógeno del carbono del hidrocarburo hacia el radical $\mathrm{OH} \bullet$, con ruptura y formación de enlaces, los cálculos se realizaron con la base $6-31 \mathrm{G}^{\star \star}$ que incluye orbitales de polarización $\mathrm{d}$ sobre los átomos de carbono y oxígeno, y orbitales p para los átomos de hidrógeno.

La reacción de oxidación de compuestos aromáticos con el radical $\mathrm{OH} \bullet$ ocurre por dos vias: la reacción de abstracción de hidrógeno del metilo por el radical $\mathrm{OH} \bullet \mathrm{y}$ la adición del radical $\mathrm{OH}$ • al anillo aromático. La reacción de abstracción es análoga a la reacción de oxidación de las parafinas, por lo que se siguió la misma metodología. Para el estudio de la reacción de adición del radical $\mathrm{OH} \bullet$ al anillo aromático para formar el radical MHCHD, así como para la reacción de este radical con $\mathrm{O}_{2}$ para formar los radicales peroxilo, y los radicales tipo epóxido, se utilizó una base más pequeña, (la $6-31 G^{\star}$ ), ya que no intervienen átomos de hidrógeno directamente. Los cálculos se realizaron utilizando los métodos B3LYP y UMP2. 
La determinación de los estados de transición presenta, en general, muchas dificultades de convergencia tanto en el SCF como en la optimización de la geometría. En este trabajo la obtención del estado de transición se realizó a través de tres etapa. Primero, se optimiza parcialmente la estructura en el espacio de control; segundo, se fija la parte optimizada anteriormente y se optimizan de manera parcial las variables del espacio ortogonal al espacio de control. Estos dos pasos se repiten, si es necesario, varias veces. Finalmente, se relajan todas las variables simultáneamente.

Una vez obtenido el estado de transición, es necesario realizar un cálculo de frecuencias de vibración, para determinar si la estructura obtenida corresponde a un estado de transición real o local. La presencia de una sola frecuencia imaginaria, indica un estado de transición real. Si se obtiene más de una frecuencia imaginaria se trata de un punto de inflexión en la superficie de energia potencial (estado de transición local).

La vizualización del modo normal de vibración correspondiente a la frecuencia imaginaria permite garantizar que el estado de transición es efectivamente el que corresponde a la coordenada de reacción buscada y que conecta los reactivos con los productos. Las estructuras optimizadas de los estado de transición calculados se visualizaron con el programa GaussView [95].

En todos los casos estudiados, por tratarse de un sistema de radicales libres con un electrón no apareado, el estado electrónico es un doblete (espin total $=1 / 2$ ). Sosa y Schlegel ${ }^{[71]}$ fueron los primeros en mostrar que, para el caso de reacciones de adición $\mathrm{OH} \bullet+\mathrm{C}_{2} \mathrm{H}_{2}$ y $\mathrm{OH} \bullet+\mathrm{C}_{2} \mathrm{H}_{4}$ (que también son dobletes), los mejores resultados para las barreras de activación se obtienen si se elimina la componente del cuarteto (espin total igual a 3/2) en la función de onda del radical. Esto equivale a utilizar las energias PMP2 calculadas automáticamente en el paquete Gaussian 94 al introducir las correcciones UMP2. La proyección del espin se realiza después de haber optimizado la geometria. La utilización de las energias proyectadas PMP2 es discutible, y sólo se justifica con base en la coherencia de los resultados obtenidos en muchos casos.

Para estimar las energias de reacción, $\Delta H_{\text {reacción, }} y$ las energias de activación $E_{a}$, de los sistemas estudiados fue necesario tomar en cuenta la corrección de la energia de punto cero (ZPE), en la energia total de estos sistemas.

La energia de punto cero, se define como la corrección a la energia que toma en cuenta los efectos vibracionales de las moléculas. Además, la energía térmica (TE) se 
define como la corrección a la energia total del sistema debida a la activación de los grados de libertad de rotación, translación y vibración a una temperatura y presión especifica. Cabe señalar que, en la energía térmica se incluye la energía de punto cero automáticamente [96-97]. En este estudio, para estimar la $\Delta \mathrm{H}_{\text {reacción }}$ y la $\mathrm{E}_{\mathrm{a}}$, se usó la corrección térmica.

\section{B.2 Recursos computacionales}

La cantidad de recursos de computo requeridos para los cálculos de estructura electrónica depende del número de funciones base. El número de funciones base se denota por $N$, mientras que el número de orbitales ocupados por $O$ y el número de orbitales virtuales por $V$.

El número de primeras derivadas con respecto a la posición de los átomos es $N_{\mathrm{a}}$ (igual a tres veces el número de átomos). La ecuación empírica (B.1) estima la cantidad de memoria necesaria para realizar cálculos con el programa Gaussian 94.

$$
\mathrm{M}+2 \mathrm{~N}_{\mathrm{B}}^{2}
$$

donde $\mathrm{N}_{\mathrm{B}}$ es el número de funciones base y $\mathrm{M}$ la cantidad minima de memoria que depende del tipo de cálculo [95].

En los cálculos de orbitales moleculares post-Hartrre-Fock se requiere evaluar integrales sobre las funciones de base. Las integrales monoelectrónicas requieren poco tiempo y espacio en disco, ya que solo hay del orden de $o\left(N^{2}\right)$. En cambio el cálculo de las integrales bielectrónicas requieren mucho tiempo y espacio en disco ya que son del orden de $o\left(N^{4}\right)$. En los cálculos con DFT el escalamiento es de orden $o(M)^{[83]}$.

En este trabajo, la optimización de las estructuras de minima energia de los reactivos y productos en la reacción de oxidación de las parafinas se obtuvieron con la opción SCF directo del programa Gaussian 94, mientras que en la optimización de las estructuras de los estados de transición, se usó la opción $\mathrm{SCF}=\mathrm{QC}$ de convergencia cuadrática [98].

En la reacción de oxidación de los compuestos aromáticos, la optimización de las estructuras de los radicales $\mathrm{OH}-\mathrm{Ar}-\mathrm{OO} \bullet \mathrm{y}$ de los radicales epóxido, presentó muchos problemas de convergencia tanto en el SCF como en la optimización de la geometria, y se 
requirieron una gran cantidad de recursos de cómputo. Para la optimización de los reactivos y productos, se usó la opción $\mathrm{SCF}=\mathrm{QC}$ (convergencia cuadrática) y en algunos caso, la opción $\mathrm{SCF}=\mathrm{DIIS} \mathrm{IOP}=(1 / 8=1)$, que reduce el desplazamiento de los âtomos en cada paso de la optimización.

\section{B.3 Estaciones de trabajo y supercomputadoras}

La cantidad de recursos computacionales requerida en este trabajo, solo fue posible a través del uso de diferentes estaciones de trabajo y supercomputadoras. El equipo utilizado es el siguiente:

Servidor Power Challenge / Silicon Graphics

Modelo XL

18 procesadores $\mathrm{R} 8000$.

47 Gibytes en disco

2 Gigabytes en RAM

Ubicación: Laboratorio de Supercomputo de la UAM-lztapalapa

Servidor Origin 200 / Silcon Graphics

2 Procesadores R10000

40 Gigabytes en disco

256 Megabytes en RAM

Ubicación: Area de Quimica Cuática de la UAM-Iztapalapa

Supercomputadora CRAY-YMP 4 /464

4 procesadores

38.4 Gigabytes en disco

SSD (Dispositivo de Almacenamiento de Estado Sólido). Este es un banco de memoria auxiliar RAM de $128 \mathrm{MW}$ (1 GB).

Ubicación: DGSCA UNAM

Origin 2000 / Silicon Graphics

32 Procesadores R10000

65 Gigabytes en disco

10 Gigabytes en RAM

Ubicación: DGSCA UNAM 


\section{B.4 Programas}

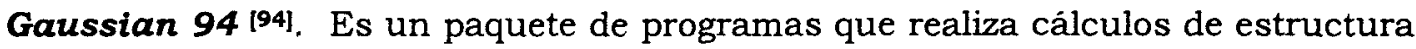
electrónica a diferentes niveles de cálculo, semiempíricos, ab-initio, y con la teoria de funcionales de la densidad. Este programa es una herramienta poderosa que puede explorar diferentes áreas de interés quimico, como mecanismos de reacción, superficies de energía potencial, etc.

Algunos tipos de cálculos que se pueden realizar con el programa Gaussian son:

- Determinación de energías totales

- Optimización de las estructuras de mínima energía

- Optimización de estructuras de estados de transición

- Determinación de frecuencias de vibración

- Determinación de desplazamientos Raman

- Determinación de orbitales moleculares

- Determinación de cargas atómicas

- Estudios con modelos que toman en cuenta el efecto del entorno

Spartan [93]. Este programa es una interfase gráfica con la que se pueden realizar cálculos de estructura electrónica, optimizando las estructuras a nivel semiempirico, abinitio y con funcionales de la densidad, asi como vizualizar los resultados obtenidos. Además, con este programa se pueden diseñar nuevas moléculas, las cuales se pueden editar para construir datos de entrada con los que se realizan cálculos ab-initio con otros programas, como el Gaussian 94.

GaussView [95]. Este programa, a diferencia del Spartan, es una interfase gráfica propia del programa Gaussian 94, con la cual se pueden vizualizar los resultados obtenidos con dicho programa. Entre las propiedades que se pueden vizualizar, se encuentran: los orbitales moleculares, las superficies de densidad electrónica, las superficies de potencial electrostático, así como los modos normales de vibración. Con este programa también se pueden diseñar nuevas moléculas, y generar datos de entrada para realizar cálculos ab-initio, con el programa Gaussian 94. 


\section{ANEXO 3}

Geometria optimizada del TS1 del metano con $\mathrm{OH}$

\begin{tabular}{|c|c|}
\hline \multicolumn{2}{|l|}{ 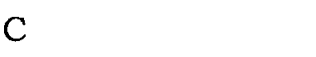 } \\
\hline \multicolumn{2}{|c|}{$\mathrm{H}, 1, \mathrm{r} 2$} \\
\hline \multicolumn{2}{|c|}{$0,2, \mathrm{r} 3,1, \mathrm{a} 3$} \\
\hline \multicolumn{2}{|c|}{$\mathrm{H}, 3, \mathrm{r} 4,2, \mathrm{a} 4,1, \mathrm{~d} 4,0$} \\
\hline \multicolumn{2}{|c|}{$\mathrm{H}, 1, \mathrm{r} 5,2, \mathrm{a} 5,4, \mathrm{~d} 5,0$} \\
\hline \multirow{2}{*}{\multicolumn{2}{|c|}{$\begin{array}{l}\mathrm{H}, 1, \mathrm{r} 6,2, \mathrm{a} 6,4, \mathrm{~d} 6,0 \\
\mathrm{H}, 1, \mathrm{r} 7,2, \mathrm{a} 7,4, \mathrm{~d} 7,0\end{array}$}} \\
\hline & \\
\hline r2 & 1.2055 \\
\hline r3 & 1.2814 \\
\hline a3 & 168.7853 \\
\hline r4 & 0.9709 \\
\hline a4 & 98.5195 \\
\hline d4 4 & 360.0504 \\
\hline r5 & 1.0837 \\
\hline a5 & 108.391 \\
\hline d5 & 360.134 \\
\hline r6 & 1.0835 \\
\hline a6 & 105.586 \\
\hline d6 & 239.865 \\
\hline $\mathrm{r} 7$ & 1.0835 \\
\hline a7 & 105.5762 \\
\hline $\mathrm{d} 7$ & 120.3955 \\
\hline
\end{tabular}




\section{Geometría optimizada del TS2 de radical metil con $\mathrm{O}_{2}$}

\begin{tabular}{lc}
$\mathrm{C}$ \\
$\mathrm{H}, 1, \mathrm{r} 2$ \\
$\mathrm{O}, 1, \mathrm{r} 3,2, \mathrm{a} 3$ \\
$\mathrm{H}, 1, \mathrm{r} 4,2, \mathrm{a} 4,3, \mathrm{~d} 4,0$ \\
$\mathrm{H}, 1, \mathrm{r} 5,2, \mathrm{a} 5,3, \mathrm{~d} 5,0$ \\
$\mathrm{O}, 3, \mathrm{r} 6,1, \mathrm{a} 6,2, \mathrm{~d} 6,0$ \\
\multicolumn{3}{c}{} \\
$\mathrm{r} 2$ & 1.0772 \\
$\mathrm{r} 3$ & 2.0096 \\
$\mathrm{a} 3$ & 98.2748 \\
$\mathrm{r} 4$ & 1.0775 \\
$\mathrm{a} 4$ & 117.694 \\
$\mathrm{~d} 4$ & 253.3222 \\
$\mathrm{r} 5$ & 1.0772 \\
$\mathrm{a} 5$ & 117.3066 \\
$\mathrm{~d} 5$ & 103.8159 \\
$\mathrm{r} 6$ & 1.2133 \\
$\mathrm{a} 6$ & 114.7332 \\
$\mathrm{~d} 6$ & 59.6374
\end{tabular}


Geometria optimizada del TS1 de etano con OH

\begin{tabular}{|c|c|}
\hline \multicolumn{2}{|c|}{$\begin{array}{l}\mathrm{C} \\
\mathrm{C}, 1, \mathrm{r} 2 \\
\mathrm{H}, 1, \mathrm{r} 3,2, \mathrm{a} 3 \\
\mathrm{H}, 1, \mathrm{r} 4,2, \mathrm{a} 4,3, \mathrm{~d} 4,0 \\
\mathrm{H}, 1, \mathrm{r} 5,2, \mathrm{a} 5,3, \mathrm{~d} 5,0 \\
\mathrm{H}, 2, \mathrm{r} 6,1, \mathrm{a} 6,3, \mathrm{~d} 6,0 \\
\mathrm{H}, 2, \mathrm{r} 7,1, \mathrm{a} 7,3, \mathrm{~d} 7,0 \\
\mathrm{H}, 2, \mathrm{r} 8,1, \mathrm{a} 8,3, \mathrm{~d} 8,0 \\
\mathrm{O}, 6, \mathrm{r} 9,2, \mathrm{a} 9,1, \mathrm{~d} 9,0 \\
\mathrm{H}, 9, \mathrm{r} 10,6, \mathrm{a} 10,7, \mathrm{~d} 10,0\end{array}$} \\
\hline \multicolumn{2}{|r|}{1.5125} \\
\hline r3 & 1.0906 \\
\hline a3 & 110.8139 \\
\hline $\mathrm{r} 4$ & 1.0879 \\
\hline $\mathrm{a} 4$ & 110.9766 \\
\hline \multirow{2}{*}{$\begin{array}{l}\mathrm{d} 4 \\
\mathrm{r} 5\end{array}$} & 119.9579 \\
\hline & 1.088 \\
\hline \multirow{2}{*}{$\begin{array}{l}\text { a5 } \\
\text { d5 }\end{array}$} & 111.0089 \\
\hline & 240.0216 \\
\hline \multirow{2}{*}{$\begin{array}{l}\text { r6 } \\
\text { a6 }\end{array}$} & 1.1886 \\
\hline & 107.6859 \\
\hline & 180.4315 \\
\hline & 1.0875 \\
\hline \multirow{2}{*}{$\begin{array}{l}\text { a7 } \\
\text { d7 }\end{array}$} & 113.9252 \\
\hline & 295.5479 \\
\hline r8 & 1.0877 \\
\hline \multirow{2}{*}{$\begin{array}{l}\text { a8 } \\
\text { d8 }\end{array}$} & 113.4825 \\
\hline & 62.5145 \\
\hline \multirow{2}{*}{$\begin{array}{l}\text { r9 } \\
\text { a9 }\end{array}$} & 1.3223 \\
\hline & 168.9518 \\
\hline \multirow{2}{*}{$\begin{array}{l}\text { d9 } \\
\text { r } 10\end{array}$} & 284.0501 \\
\hline & 0.9713 \\
\hline \multirow{2}{*}{$\begin{array}{l}\text { a } 10 \\
\text { d10 }\end{array}$} & 97.596 \\
\hline & 114.5106 \\
\hline
\end{tabular}




\section{Geometria optimizada del TS2 de radical etil con $\mathrm{O}_{2}$}

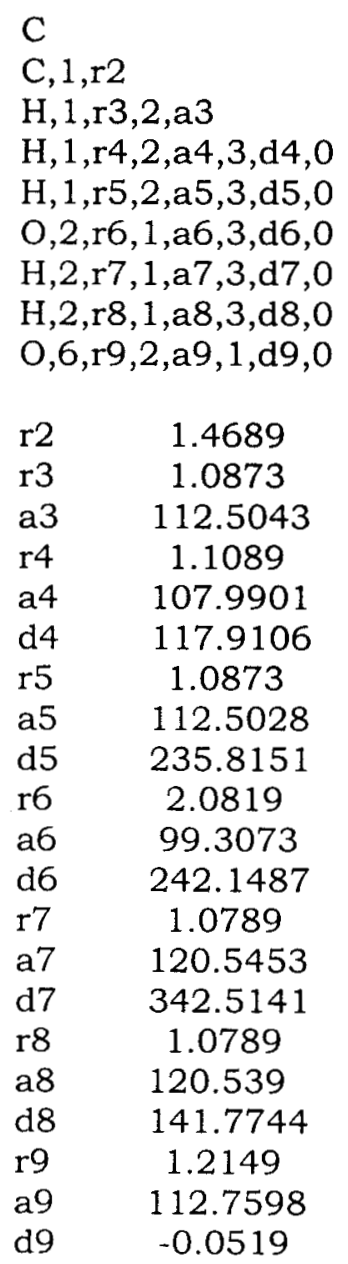




\section{Geometria optimizada del TS1 de propano (H primario) con OH}

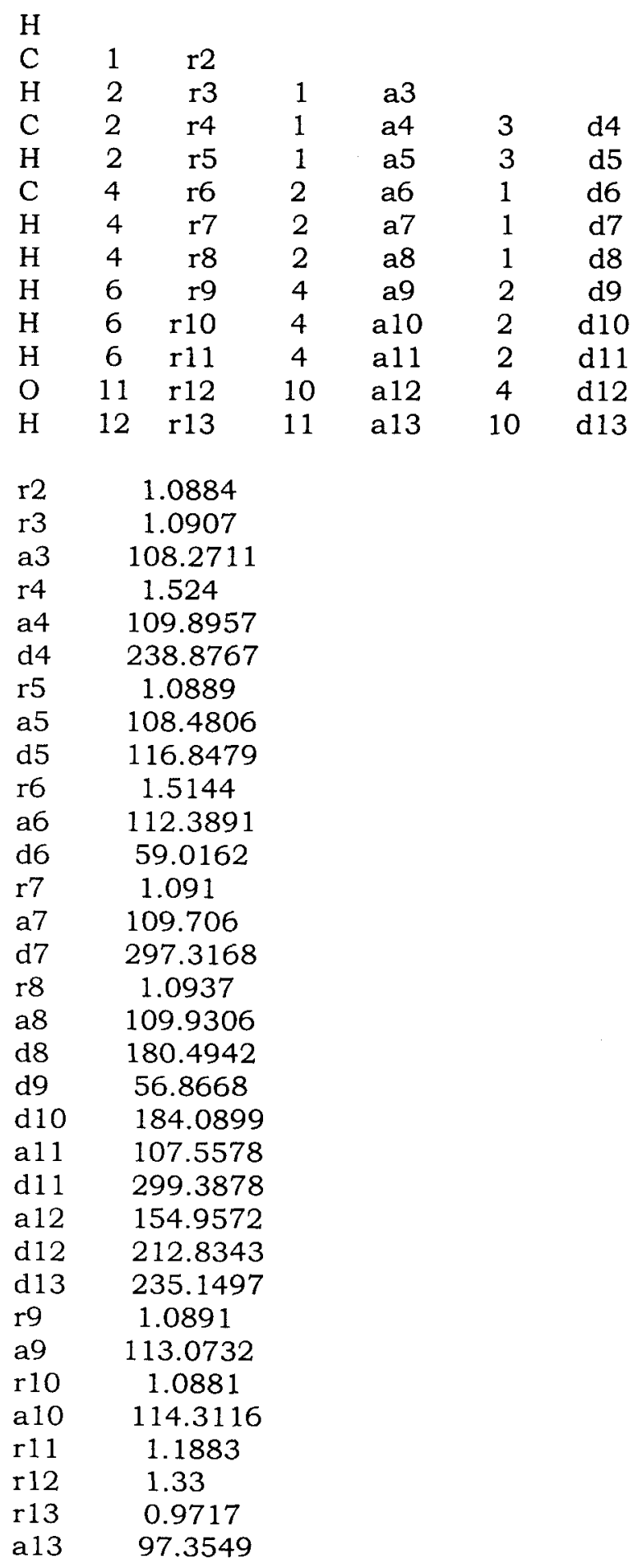




\section{Geometria optimizada del TS2 de radical propil primario con $\mathrm{O}_{2}$}

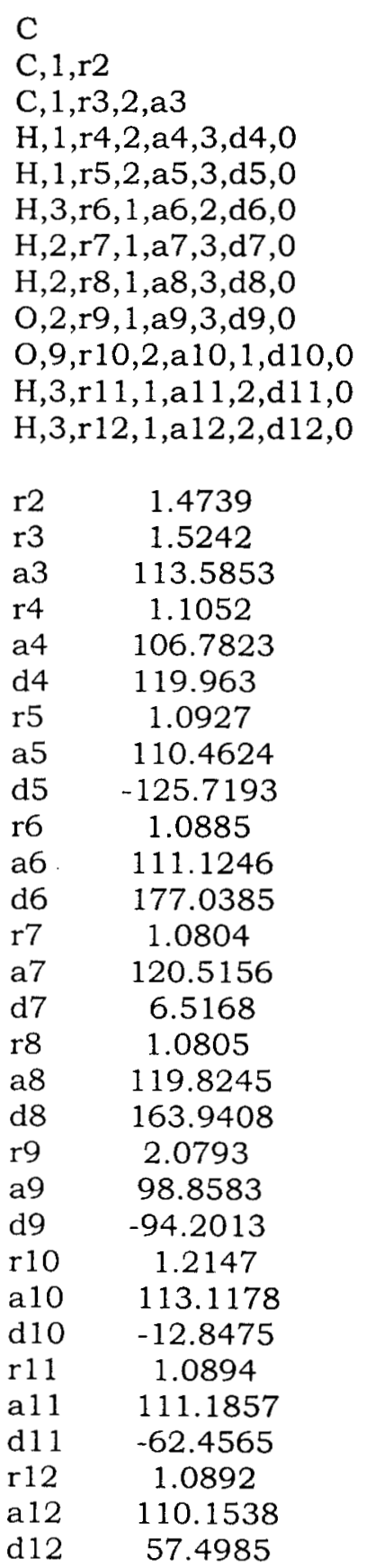


Geometría optimizada del TS1 de propano (H secundario) con OH

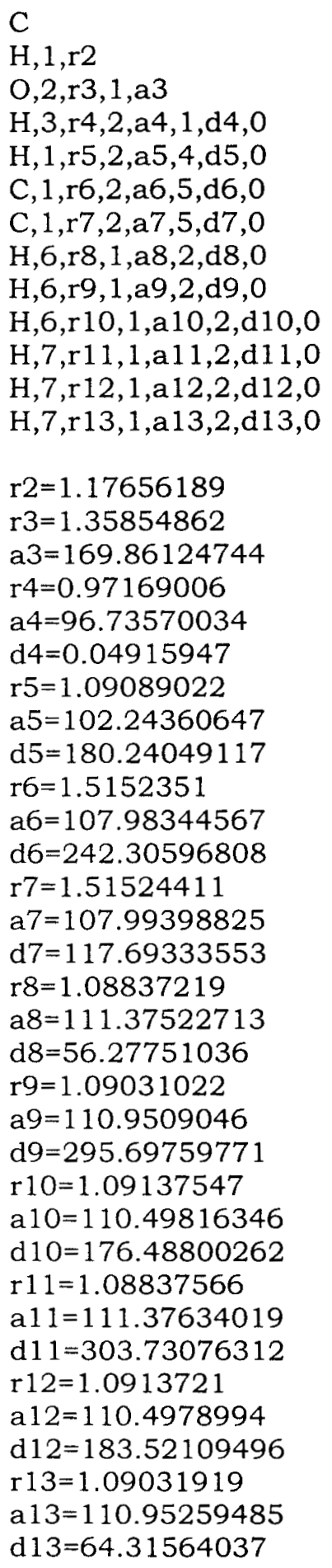


Geometria optimizada del TS2 de radical propil secundario con $\mathrm{O}_{2}$

\begin{tabular}{|c|c|}
\hline \multicolumn{2}{|c|}{$\begin{array}{l}\mathrm{H}, 1, \mathrm{r} 2 \\
\mathrm{O}, 1, \mathrm{r} 3,2, \mathrm{a} 3 \\
\mathrm{O}, 3, \mathrm{r} 4,1, \mathrm{a} 4,2, \mathrm{~d} 4,0 \\
\mathrm{C}, 1, \mathrm{r} 5,2, \mathrm{a} 5,3, \mathrm{~d} 5,0 \\
\mathrm{C}, 1, \mathrm{r} 6,2, \mathrm{a} 6,3, \mathrm{~d} 6,0 \\
\mathrm{H}, 6, \mathrm{r} 7,1, \mathrm{a} 7,2, \mathrm{~d} 7,0 \\
\mathrm{H}, 6, \mathrm{r} 8,1, \mathrm{a} 8,2, \mathrm{~d} 8,0 \\
\mathrm{H}, 6, \mathrm{r} 9,1, \mathrm{a} 9,2, \mathrm{~d} 9,0 \\
\mathrm{H}, 5, \mathrm{r} 10,1, \mathrm{a} 10,2, \mathrm{~d} 10,0 \\
\mathrm{H}, 5, \mathrm{r} 11,1, \mathrm{a} 11,2, \mathrm{~d} 11,0 \\
\mathrm{H}, 5, \mathrm{r} 12,1, \mathrm{a} 12,2, \mathrm{~d} 12,0\end{array}$} \\
\hline $\mathrm{r} 2$ & 1.0813 \\
\hline r3 & 2.1306 \\
\hline a3 & 93.1619 \\
\hline $\mathrm{r} 4$ & 1.215 \\
\hline a4 & 112.8987 \\
\hline $\mathrm{d} 4$ & 232.5649 \\
\hline r5 & 1.4708 \\
\hline a5 & 118.8404 \\
\hline d5 & 259.2744 \\
\hline r6 & 1.4896 \\
\hline a6 & 117.4918 \\
\hline d6 & 98.5333 \\
\hline $\mathrm{r} 7$ & 1.0942 \\
\hline a7 & 110.903 \\
\hline $\mathrm{d} 7$ & 80.1262 \\
\hline r8 & 1.0888 \\
\hline a8 & 111.5301 \\
\hline $\mathrm{d} 8$ & 320.0751 \\
\hline r9 & 1.0899 \\
\hline a9 & 110.4899 \\
\hline d9 & 199.2393 \\
\hline r 10 & 1.1091 \\
\hline a 10 & 108.2349 \\
\hline $\mathrm{d} 10$ & 106.4506 \\
\hline r11 & 1.0874 \\
\hline a 11 & 112.7372 \\
\hline d 11 & 347.9655 \\
\hline $\mathrm{r} 12$ & 1.0897 \\
\hline a 12 & 112.1416 \\
\hline $\mathrm{d} 12$ & 224.0766 \\
\hline
\end{tabular}


Geometria optimizada del TS1 de butano (H primario) con OH

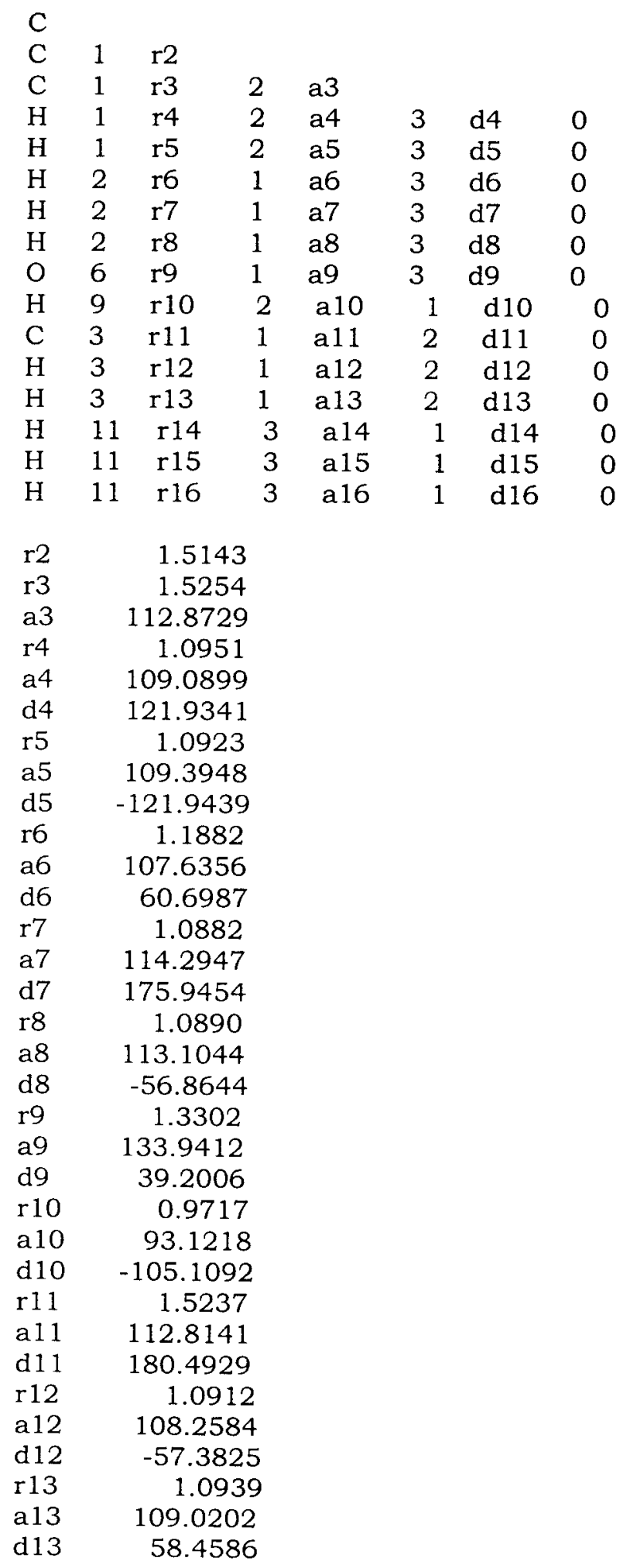



r14 $\quad 1.0893$
a14 111.3994
d14 - 180.0915
r15 1.0903
a15 110.9369
d15 -59.8421
r16 $\quad 1.0900$
a16 110.7377
d16 $\quad 59.8431$ 


\section{Geometria optimizada del TS2 de radical butil primario con $\mathrm{O}_{2}$}

$\begin{array}{lc}\mathrm{C} & \\ \mathrm{C}, 1, \mathrm{r} 2 & \\ \mathrm{C}, 1, \mathrm{r} 3,2, \mathrm{a} 3 \\ \mathrm{H}, 1, \mathrm{r} 4,2, \mathrm{a} 4,3, \mathrm{~d} 4,0 \\ \mathrm{H}, 1, \mathrm{r} 5,2, \mathrm{a} 5,3, \mathrm{~d} 5,0 \\ \mathrm{C}, 3, \mathrm{r} 6,1, \mathrm{a} 6,2, \mathrm{~d} 6,0 \\ \mathrm{H}, 2, \mathrm{r} 7,1, \mathrm{a} 7,3, \mathrm{~d} 7,0 \\ \mathrm{H}, 2, \mathrm{r} 8,1, \mathrm{a} 8,3, \mathrm{~d} 8,0 \\ \mathrm{O}, 2, \mathrm{r} 9,1, \mathrm{a} 9,3, \mathrm{~d} 9,0 \\ \mathrm{O}, 9, \mathrm{r} 10,2, \mathrm{a} 10,1, \mathrm{~d} 10,0 \\ \mathrm{H}, 6, \mathrm{r} 11,3, \mathrm{a} 11,1, \mathrm{~d} 11,0 \\ \mathrm{H}, 3, \mathrm{r} 12,1, \mathrm{a} 12,2, \mathrm{~d} 12,0 \\ \mathrm{H}, 3, \mathrm{r} 13,1, \mathrm{a} 13,2, \mathrm{~d} 13,0 \\ \mathrm{H}, 6, \mathrm{r} 14,3, \mathrm{a} 14,1, \mathrm{~d} 14,0 \\ \mathrm{H}, 6, \mathrm{r} 15,3, \mathrm{a} 15,1, \mathrm{~d} 15,0 \\ & \\ \mathrm{r} 2 & 1.4732 \\ \mathrm{r} 3 & 1.5259 \\ \mathrm{a} 3 & 113.9421 \\ \mathrm{r} 4 & 1.1066 \\ \mathrm{a} 4 & 106.9434 \\ \mathrm{~d} 4 & 119.6948 \\ \mathrm{r} 5 & 1.094 \\ \mathrm{a} 5 & 110.7779 \\ \text { d5 } & 234.2406 \\ \mathrm{r} 6 & 1.5233 \\ \mathrm{a} 6 & 112.5191 \\ \text { d6 } & 176.2668 \\ \mathrm{r} 7 & 1.0805 \\ \mathrm{a} 7 & 120.4338 \\ \text { d7 } & 366.2294 \\ \mathrm{r} 8 & 1.0805 \\ \mathrm{a} 8 & 119.9386 \\ \mathrm{~d} 8 & 163.7004 \\ \mathrm{r} 9 & 2.0805 \\ \mathrm{a} 9 & 98.7256 \\ \mathrm{~d} 9 & 265.7667 \\ \mathrm{r} 10 & 1.2147 \\ \mathrm{a} 10 & 113.0935 \\ \mathrm{~d} 10 & 347.7352 \\ \mathrm{r} 11 & 1.0902 \\ \mathrm{a} 11 & 110.8681 \\ \mathrm{~d} 11 & 60.0572 \\ \mathrm{r} 12 & 1.0925 \\ \mathrm{a} 12 & 109.4532 \\ \mathrm{~d} 12 & 298.7237 \\ \mathrm{r} 13 & 1.0923 \\ \mathrm{a} 13 & 108.4317 \\ \mathrm{~d} 13 & 54.7965 \\ \mathrm{r} 14 & 1.0891 \\ & \end{array}$


a14 111.3556

d14 180.2198

r15 1.0898

a15 110.6752

d15 300.3791 


\section{Geometria optimizada del TS1 de butano (H secundario) con OH}

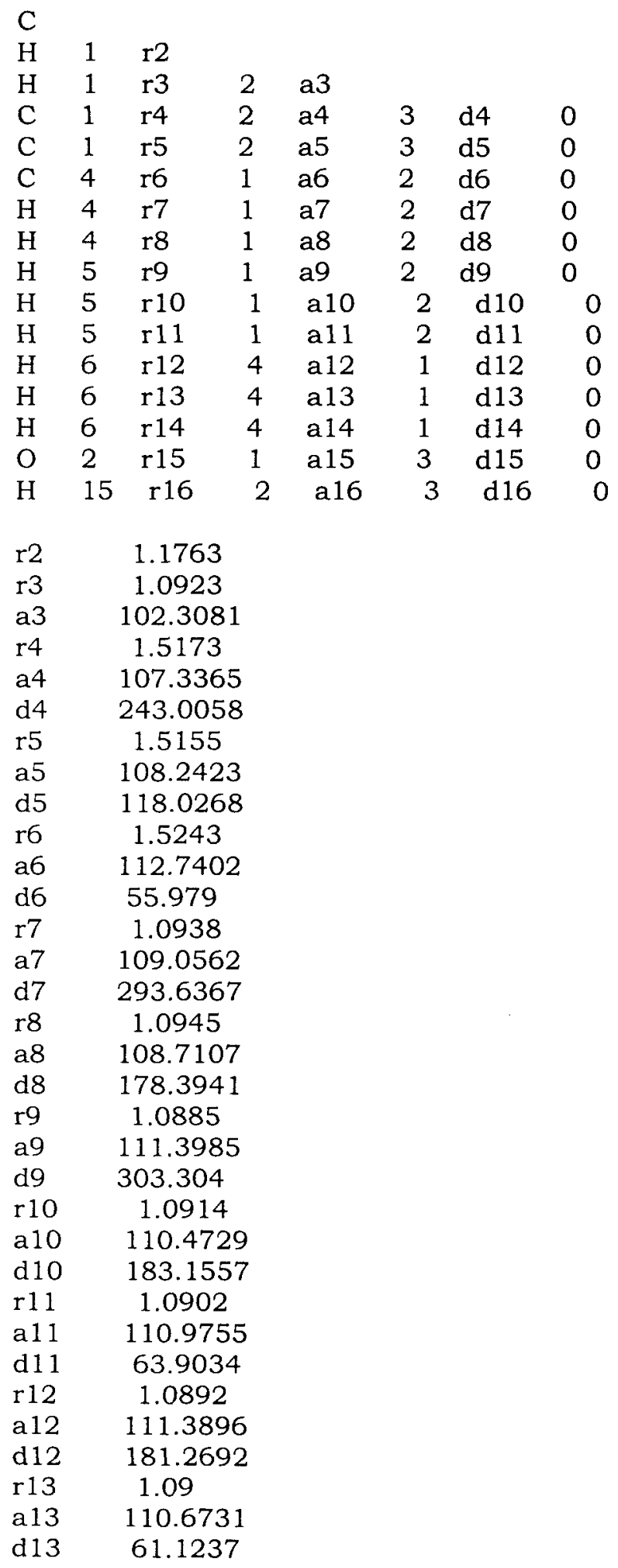



r14 1.0885
a14 110.0924
d14 301.9066
r15 1.3671
a15 168.9662
d15 165.2199
r16 0.9721
a16 96.4004
d16 174.4551 
Geometria optimizada del TS2 de radical butil secundario con $\mathrm{O}_{2}$

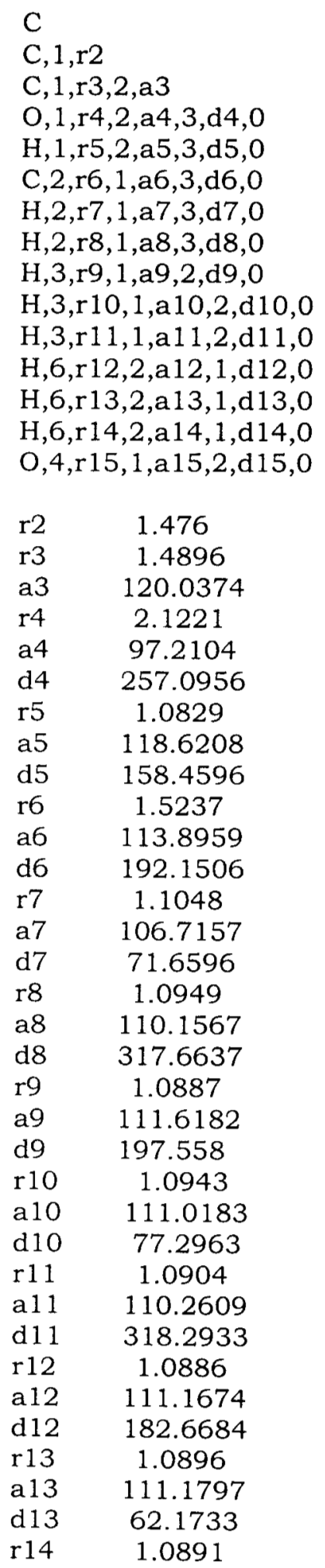



a14 110.1229
d14 302.2317
r15 1.2145
a15 113.0407
d15 20.8522 
Geometria optimizada del TS1 de iso-butano (H primario) con OH

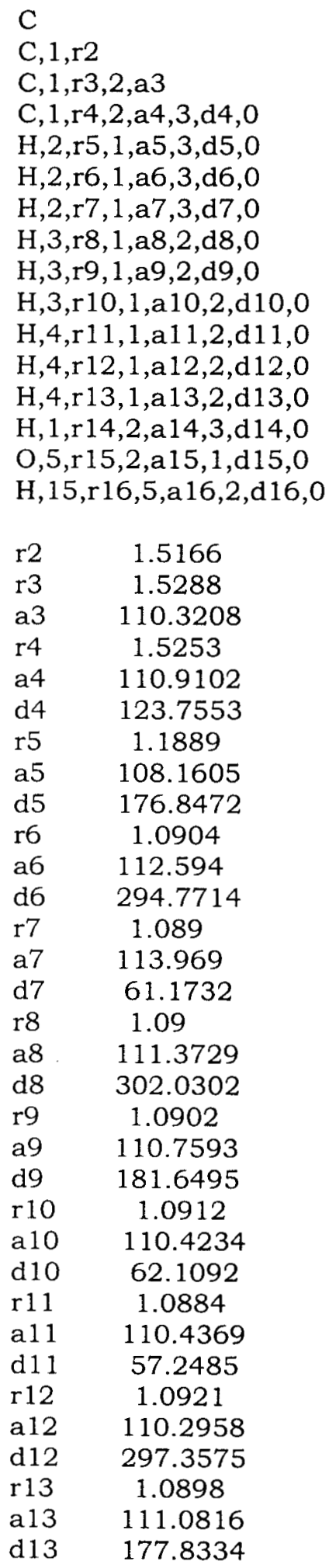



r14 $\quad 1.0937$
a14 107.8015
d14 242.0447
r15 1.3284
a15 168.866
d15 74.9061
r16 0.9717
a16 97.3521
d16 35.1429 
Geometria optimizada del TS2 de radical iso-butil primario con $\mathrm{O}_{2}$

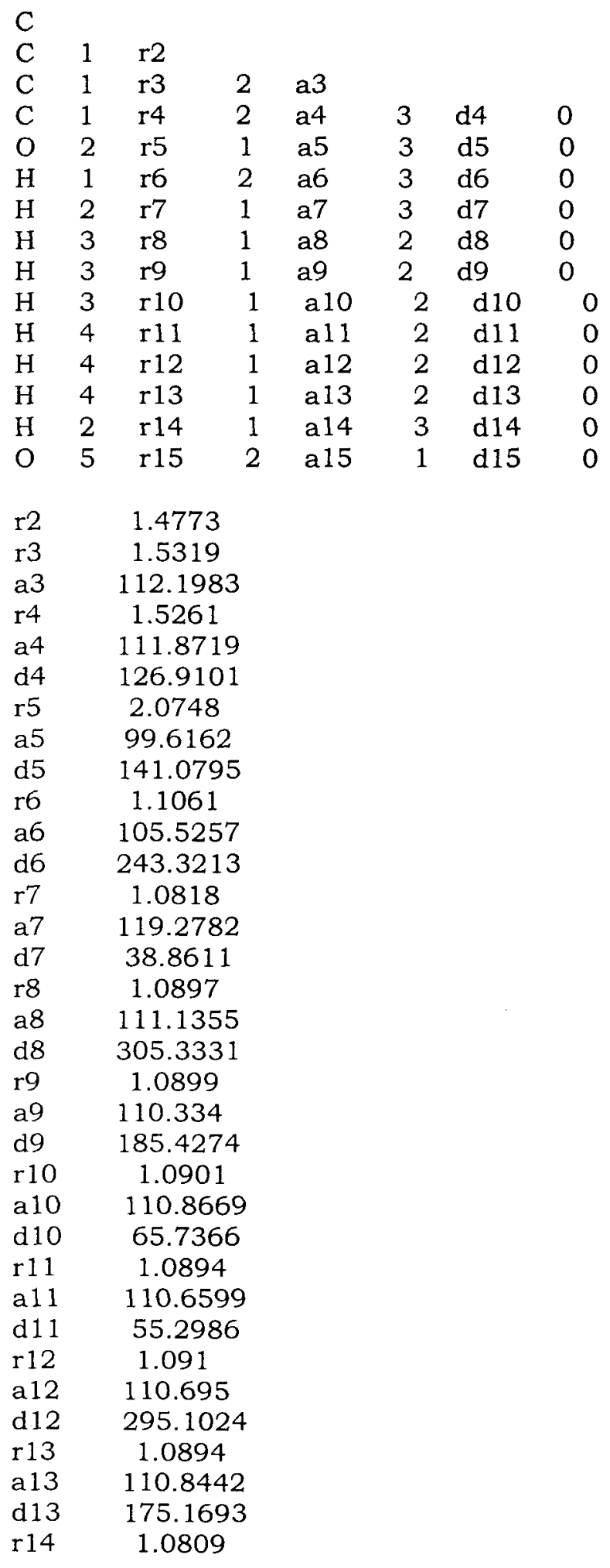



a14 120.8157
d14 242.3595
r15 1.2146
a15 113.5315
d15 347.29 


\section{Geometria optimizada del TS1 de iso-butano (H terciario) con OH}

$\begin{array}{llllllll}\mathrm{C} & & & & & & & \\ \mathrm{H} & 1 & \mathrm{r} 2 & & & & & \\ \mathrm{O} & 2 & \mathrm{r} 3 & 1 & \mathrm{a} 3 & & & \\ \mathrm{H} & 3 & \mathrm{r} 4 & 2 & \mathrm{a} 4 & 1 & \mathrm{~d} 4 & 0 \\ \mathrm{C} & 1 & \mathrm{r} 5 & 2 & \mathrm{a} 5 & 4 & \mathrm{~d} 5 & 0 \\ \mathrm{C} & 1 & \mathrm{r} 6 & 2 & \mathrm{a} 6 & 4 & \mathrm{~d} 6 & 0 \\ \mathrm{C} & 1 & \mathrm{r} 7 & 2 & \mathrm{a} 7 & 4 & \mathrm{~d} 7 & 0 \\ \mathrm{H} & 6 & \mathrm{r} 8 & 1 & \mathrm{a} 8 & 2 & \mathrm{~d} 8 & 0 \\ \mathrm{H} & 6 & \mathrm{r} 9 & 1 & \mathrm{a} 9 & 2 & \mathrm{~d} 9 & 0 \\ \mathrm{H} & 6 & \mathrm{r} 10 & 1 & \mathrm{a} 10 & 2 & \mathrm{~d} 10 & 0 \\ \mathrm{H} & 7 & \mathrm{r} 11 & 1 & \mathrm{a} 11 & 2 & \mathrm{~d} 11 & 0 \\ \mathrm{H} & 7 & \mathrm{r} 12 & 1 & \mathrm{a} 12 & 2 & \mathrm{~d} 12 & 0 \\ \mathrm{H} & 7 & \mathrm{r} 13 & 1 & \mathrm{a} 13 & 2 & \mathrm{~d} 13 & 0 \\ \mathrm{H} & 5 & \mathrm{r} 14 & 1 & \mathrm{a} 14 & 2 & \mathrm{~d} 14 & 0 \\ \mathrm{H} & 5 & \mathrm{r} 15 & 1 & \mathrm{a} 15 & 2 & \mathrm{~d} 15 & 0 \\ \mathrm{H} & 5 & \mathrm{r} 16 & 1 & \mathrm{a} 16 & 2 & \mathrm{~d} 16 & 0\end{array}$

r2 1.1662

r3 1.3925

a3 175.1439

r4 0.9719

a4 96.1342

d4 0.1189

r5 $\quad 1.5187$

a5 104.5824

d5 179.9335

r6 1.5184

a6 106.8361

d6 $\quad 60.4104$

r7 $\quad 1.5184$

a7 $\quad 106.8345$

d7 299.457

r8 $\quad 1.0893$

a8 111.0796

d8 $\quad 58.2138$

r9 1.0903

a9 111.4223

d9 297.3567

r10 1.0925

a10 $\quad 110.0617$

d10 177.8933

r11 1.0893

a11 111.0796

d11 301.7846

r12 1.0925

a12 110.0616

d12 182.1048

r13 1.0903

a13 111.4219

d13 62.6408 

r14 1.0929
a14 110.0661
d14 180.0003
r15 1.0893
a15 111.043
d15 60.2777
r16 1.0893
a16 111.0429
d16 299.7228 


\section{Geometria optimizada del TS2 de radical iso-butil terciario con $\mathrm{O}_{2}$}

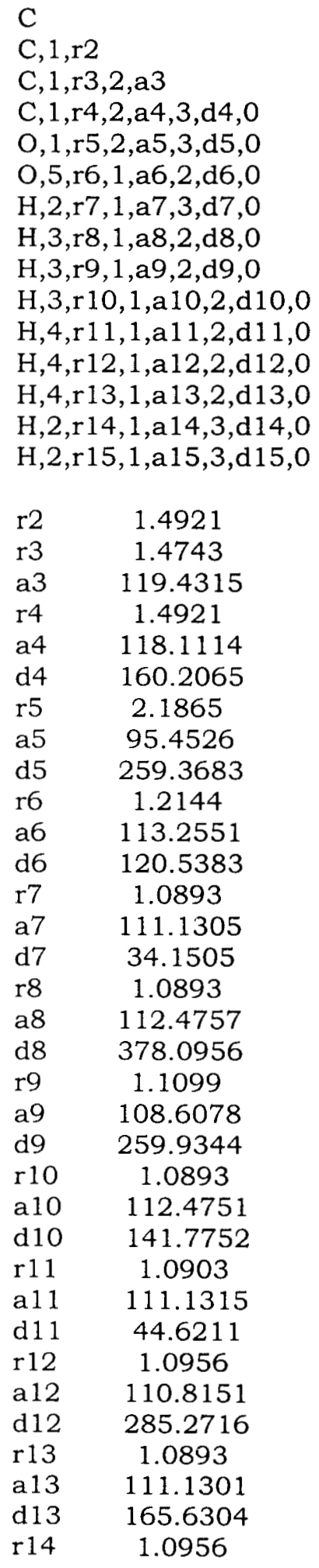


a14 110.8157

d14 274.5081

r15 1.0903

a15 111.1307

d15 155.1594 


\section{Geometria optimizada del TS1 de neo-pentano con OH}

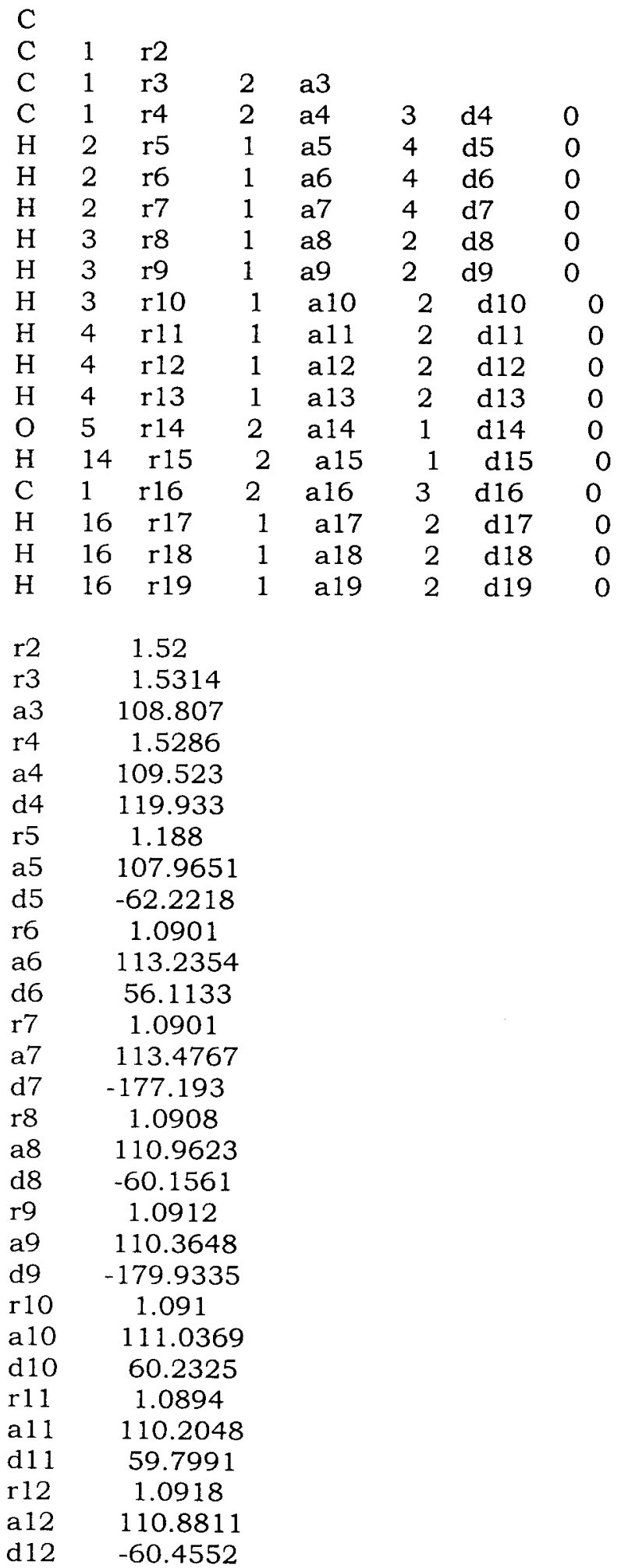




$\begin{array}{lc}\text { r13 } & 1.0907 \\ \text { a13 } & 110.6999 \\ \text { d13 } & 179.7473 \\ \text { r14 } & 1.3335 \\ \text { a14 } & 169.1973 \\ \text { d14 } & 67.1735 \\ \text { r15 } & 0.9719 \\ \text { a15 } & 93.1144 \\ \text { d15 } & 102.2953 \\ \text { r16 } & 1.5286 \\ \text { a16 } & 109.3117 \\ \text { d16 } & -119.9538 \\ \text { r17 } & 1.0898 \\ \text { a17 } & 110.4542 \\ \text { d17 } & -59.8791 \\ \text { r18 } & 1.0912 \\ \text { a18 } & 110.8173 \\ \text { d18 } & 60.161 \\ \text { r19 } & 1.0909 \\ \text { a19 } & 110.608 \\ \text { d19 } & 179.9636\end{array}$


Geometria optimizada del TS2 de radical neo-pentil con $\mathrm{O}_{2}$

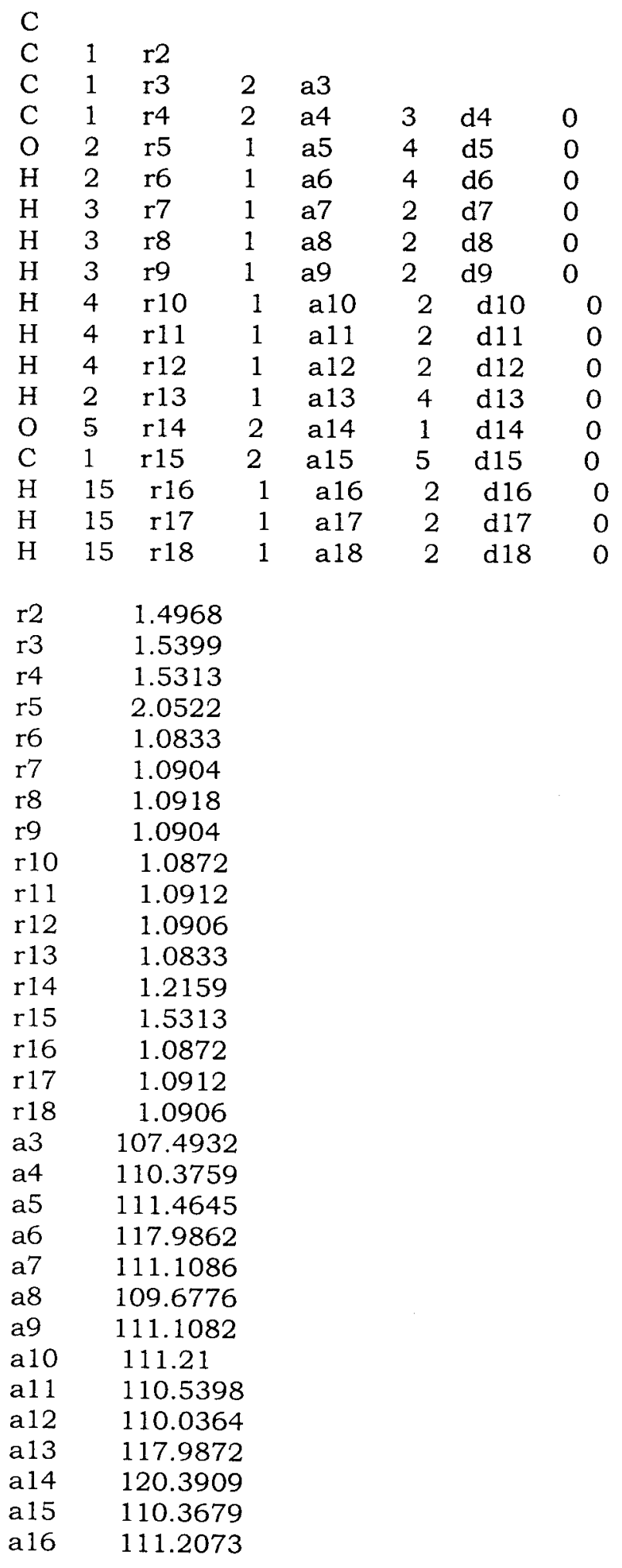



a17 $\quad 110.5392$
a18 110.0378
d4 118.6938
d5 -61.2651
d6 191.5823
d7 $\quad-60.3762$
d8 $\quad-179.9852$
d9 60.4056
d10 62.3162
d11 -58.3622
d12 182.4487
d13 45.863
d $14 \quad-0.1585$
d15 61.3488
d16 -62.2896
d17 58.3879
d18 -182.4222 


\section{BIBLIOGRAFIA}

1. R. Atkinson and A. C. Lloyd. J. Phys. Chem. Ref. Data. 13, 315, (1984).

2. J. J. P. Stewart. J. Comp. Chem. 10, 209, (1989).

3. I. Garcia, V. Uc, A. Vivier-Bunge, M. E. Ruiz. Resultados sin publicar, (1996).

4. I. Garcia-Cruz, M. E. Ruiz-Santoyo, J. R. Alvarez-Idaboy and A. Vivier-Bunge. J. Comp. Chem. 20, 845, (1999).

5. D. R. Blake and F. S. Rowland. Science 269, 953, (1995).

6. a) I. García, V. Uc, M. E. Ruiz, Y. G. Smeyers, A. Vivier-Bunge. J. Mol. Struct. (Theochem) 340, 149, (1995); b) I. Garcia-Cruz, M. Castro and A. Vivier-Bunge. J. Comp. Chem. (1999), (Sometido).

7. V. Uc, I. Garcia-Cruz, Y. G. Smeyers, A. Vivier-Bunge. Fol. Chim. Theor. XXII, 161, (1994).

8. J. H. Seinfeld. "Atmospheric Chemistry and Physics of Air Pollution". WileyInterscience Publication, New York 1986.

9. J. Heicklen. "Atmospheric Chemistry". Academic Press, USA, 1976.

10. R. B. Appel, E. M. Hoffer, E. L. Kothny, S. M. Wall, M. Haik, and R. L. Knights. Environ. Sci. Technol. 13, 283, (1979).

11. C. W. Louw, J. F. Richards and P. K. Fore. Atmos. Environ. 11, 703, (1979).

12. J. L. Arriaga, M. E. Ruiz and I. Garcia. Atmosfera 9, 119, (1996).

13. W. T. Winberry Jr, N. T. Murphy, and R. M. Riggin. "Compendium of Methods for the Determination of Toxic Organic Compounds in Ambient Air". EPA-600/4-89018. U. S. Environmental Protection Agency. Research Triangle Park, North Carolina, 1988.

14. National Research Council. "Rethinking the Ozone Problem in Urban and Regional Air Pollution". National Academic Press. Washington, D. C. USA 1991

15. a) A. J. Haagen-Smit. Ind. Eng. Chem. 44, 1342, (1952); b) A. J. Haagen-Smit, C. E. Bradley and M. M. Fox. Ind. Eng. Chem. 45, 2086, (1953); c) A. J. HaagenSmit, and M. M. Fox. J. Air Pollut. Control Assoc. 4, 105, (1954); d) A. J. HaagenSmit, and M. M. Fox. SAE Trans. 63, 575, (1955).

16. E. T. Wilkins. J. R. Sanitary Inst. 74, 1, (1954)

17. C. Baird. "Environmental Chemestry". W. H- and Company, N. Y. 1995.

18. "Programa Integral Contra la Contaminación Ambiental (PICCA)". Comisión Metropolitana Para la Prevención y el Control de la Contaminación Ambiental del Valle de México. Departamento del Distrito Federal, México, 1990.

19. J. H. Seinfeld. J. Air. Pollut. Control Assoc. 38, 616, (1988). 
20. W. P. L. Carter, and R. Atkinson. Environ. Sci. Technol. 23, 864, (1989).

21. W. P. L. Carter. J. Air \& Waste Manage. Assoc. 44, 881, (1994).

22. W. P. L. Carter. "Development of Ozone Reactivity Scales for Volatile Organic Compounds". Office of Research and Development, U. S. Environmental Protection Agency, 1991.

23. W. P. L. Carter. Atmos. Environ. 29, 2513, (1995).

24. W. P. L. Carter, J. A. Pierce, D. Luo and I. L. Malkina. Atmos. Environ. 29, 2499, (1995).

25. B. J. Finlayson-Pitts, and J. N. Pitts. "Atmospheric Chemistry: Fundamentals and Experimental Techniques". Wiley-Interscience Publication. New York, 1986.

26. R. Atkinson. J. Phys. Chem. Ref. Data 26, 215 (1997)

27. J. A. Russel. J. Am. Chem. Soc. 79, 3871, (1957).

28. P. D. Bartlet and G. Guaraldi. J. Am. Chem. Soc. 89, 3871, (1967).

29. T. Minato, S. Yamabe, H. Fujimoto and K. Fukui. Bull. Chem. Soc. Jpn. 51, 682, (1978).

30. J. J. Kaufman, J. J. Harkis, and W. S. Koski. Int. J. Quantum Chem. Suppl. 1, 261, (1967).

31. K. Morokuma, and R. E. Davis. J. Am. Chem. Soc. 94, 1060, (1972).

32. M. G. Sana, G. Leroy, and J. L. Villaveces. Theoret. Chim. Acta (Berl.) 65, 109, (1984).

33. E. P. Wigner. Z. Phys. Chem. B19, 203, (1932).

34. J. M. Lluch, J. Bertran and J. J. Dannenberg. Tetrahedron 44, 7621, (1988).

35. A. E. Dorigo, and K. N. Houk. J. Org. Chem. 53, 1650, (1988).

36. R. A. Whiteside, J. S. Brinkley, R. Krishman, D. J. DeFrees, H. B. Schlegel, and J. A Pople. "Carnegie-Mellon Quantum Chemistry Archive". Carnegie-Mellon University, Pittsburgh, P. A. 1978.

37. T. N. Truong, and D. G. Truhlar. J. Chem. Phys. 93, 1761, (1990).

38. C. Gonzalez, J. J. W. McDouall, and H. B. Schlegel. J. Phys. Chem. 94, 7467, (1990).

39. V. S. Melissas, and D. G. Truhlar. J. Chem. Phys. 99, 1013, (1993).

40. S. Gordon, and D. G. Truhlar. J. Am. Chem. Soc. 108, 5412, (1986).

41. A. Gonzales-Lafont, T. N. Truong and D. G. Truhlar. J. Chem. Phys. 95, 8875, (1991).

42. V. S. Melissas, and D. G. Truhlar. J. Phys. Chem. 98, 875, (1994).

43. S. Sekusak, K. R. Liedl, B. M. Rode and A. Sabljic. J. Phys. Chem. A 101, 4245, (1997). 
44. W. P. Hu, I. Rossi, J. C. Corchado, and D. G. Truhlar. J. Phys. Chem. A 101, 6911, (1997).

45. T. P. W. Jungkamp, J. N. Smith and J. H. Seinfeld. J. Phys. Chem. A 101, 4392, (1997).

46. I. Aliagas and S. Gronert. J. Phys. Chem. A 102, 2609, (1998).

47. S. Benson. "Thermochemical Kinetics", 2nd ed. Wiley, New York, 1976.

48. P. D. Lightfoot, R. A. Cox, J. N. Crowley, M. Destriau, G. D. Hayman, M. E. Jenkin, G. K. Moorgat and F. Sabel. Atmos. Environ. 26 A, 1805, (1992).

49. S. L. Boyd, R. J. Boyd and L. Ross C. Barclay. J. Am. Chem. Soc. 112, 5724, (1990).

50. J. A. Jafri and D. H. Phillips. J. Am. Chem. Soc. 112, 2586, (1990).

51. S. D. Wetmore and R. J. Boyd. J. Chem. Phys. 106, 7738, (1997)

52. K. Tanaka, S. Sokai, S. Tomiyama, T. Nishiyama and F. Yamada. Bull. Chem. Soc. Jpn. 64, 2677, (1991).

53. L. J. Bartolotti and E. O. Edney. Chem. Phys. Lett. 245, 119, (1995).

54. J. M. Andino, J. N. Smith, R. C. Flagan, W. A. Goddard III, J. H. Seinfeld. J. Phys, Chem. 100, 10967, (1996).

55. G. Ghido and G. Tonachini. J. Am. Chem. Soc. 120, 6753, (1998).

56. B. E. Dumdei and R. J. O’Brien. Nature 311, 248, (1984)

57. R. Atkinson. Atmos. Environ. 24 A, 1, (1990).

58. R. Atkinson. J. Phys. Chem. Ref. Data Monograph 2, 1, (1994).

59. R. Atkinson. Chem. Rev. 85, 69, (1986).

60. R. Atkinson. J. Phys. Chem. Ref. Data Monograph 1, 1, (1989).

61. R. Atkinson. J. Phys. Chem. Ref. Data 20, 459 (1991).

62. R. Atkinson, D. L. Baulch, R. A. Cox, R. F. Hampson Jr. J. A. Ker and J. Troe. J. Phys. Chem. Ref. Data Monograph 1, 881, (1989).

63. W. P. L. Carter and R. Atkinson. J. Atmos. Chem. 3, 377, (1985).

64. R. Perry, R. Atkinson, and J. N. Pitts, Jr. J. Phys. Chem. 81, 296, (1977).

65. K. Lorentz and R. Zellner. Ber. Bunenges. Phys. Chem. 87, 629, (1983).

66. R. Knispel, R. Koch, M. Siese, and C. Zetzsch. Ber. Bunsenges. Phys. Chem. 94, 1375, (1990).

67. B. Klotz, I. Barnes, K. H. Becker, B. T. Golding. J. Chem. Soc. Faraday Trans. 93, 1507, (1997).

68. B. Klotz, I. Barnes, K. H. Becker. Chem. Phys. Lett. 231, 289, (1998).

69. A. S. Szabo, and N. S. Ostlund. "Modern Quantum Chemistry. Introduction to Avanced Electronic Structure Theory". Dover Publications. New York, 1996. 
70. F. L. Pilar. "Elementary Quantum Chemistry". McGraw-Hill Publishing Co. New York, 1990.

71. C. Sosa and H. B. Schlegel, J. Am. Chem. Soc. 109, 4193, (1987).

72. C. Sosa and H. B. Schlegel, Int. J. Quant. Chem. XXIX, 1001, (1986).

73. H. B. Schlegel, J. Chem. Phys. 88, 4530, (1986).

74. W. Chen and H. B. Schlegel, J. Chem. Phys. 101, 5957, (1994).

75. P. Hohenberg and W. Khon. Phys. Rev. B. 136, 864, (1964)

76. W. Yang and R. G. Parr. "Density Functional Theory of Atoms and Molecules". Oxford. New York, 1989.

77. W. Kohn and L. J. Sham. Phys. Rev. A, 1113, (1965).

78. a) DeMon-KS. D. R. Salahub, R. Fornier, P. M. Lynarsky, I. Papai, A. St-Amant and J. Ushio: in Desity Functional Methods in Chemistry. Edited by J. Labanowski and J. Andselm. Spirnger, New York, 1991. b) A. St-Amant and D. R. Salahub. Chem. Phys. Lett. 169, 387, (1990)

79. Dgauss Program. Cray Research Inc. Dgauss 3.0.1/UC-3.0, Eagan, MN, 1993.

80. ADF 2.0. User's Guide. G. Te Velde. Theoretical Chemistry Urije Universiteit. The Netherlands, 1995.

81. T. Ziegler. Chem. Rev. 91, 651, (1991).

82. J. C. Slater. Phys. Rev. 81, 385, (1951)

83. V. Barone, C. Adamo and N. Russo. Chem. Phys. Lett. 212, 5, (1993)

84. P. J. Stephens, F. J. Devlin, C. F. Chabalowski and M. J. Frisch. J. Phys. Chem. 98, 11624, (1994).

85. M. J. Cohen, and D. P. Chong. Chem. Rev. 234,405, (1995)

86. W. Chan, I. P. Hamilton, and, H. O. Pritchard. J. Chem Soc. Faraday Trans. 16, 2303, (1998).

87. I. S. Ignatyev, Y. Xie, W. D. Allen, H. F. Schaefer III. J. Chem. Phys. 107, 141, (1997).

88. I. Levine. "Quimica Cuántica". AC Ediciones. Madrid, 1989.

89. I. Shavitt, I., J. Chem. Phys. 49, 4048, (1968).

90. M. Menzinger and R. Wolfgang. Angew. Chem. Int. Ed. Engl. 8, 438, (1969).

91. W. J. Hehre, L. Redom, P. R. Shleyer and J. A Pople. "Ab Initio Molecular Orbital Theory". Wiley Interscience. New York, 1985.

92. W. J. Hehre. "Practical Strategies for Electronic Structure Calculations". California, USA, 1995.

93. Programa Spartan. W. J. Hehre. Wavefuntion, 1993.

94. Programa Gaussian94. Frisch, M. J., Trucks, G.W., Schlegel, H.B., Gill, P.M.W., Johnson, B.G., Robb, M.A., Cheeseman, J.R., Keith, T.A., Petersson, G.A., 
Montgomery, J.A., Raghavachari, K., Al-Laham, M.A., Zakrzewski, V.G., Ortiz, J.V., Foresman, J.B., Cioslowski, J., Stefanov, B.B., Nanayakkara, A., Challacombe, M., Peng, C.Y., Ayala, P.Y., Chen, W., Wong, M.W., Andres, J.L., Replogle, E.S., Gomperts, R., Martin, R.L., Fox, D.J., Binkley, J.S., Defrees, D.J., Baker, J., Stewart, J.P., Head-Gordon, M., Gonzalez, C., and Pople, J.A. Gaussian, Inc., Pittsburgh, PA, 1995.

95. Programa GaussView. A. B. Nielsen and A. J. Holder. Gaussian, Inc., Pittsburgh, PA, 1995.

96. J. B. Foresman and A. Frisch. "Exploring Chemistry with Electronic Structure Methods". California, USA, 1995. Gaussian, Inc., Pittsburgh, PA, 1995.

97. D. A. McQuarrie. "Statistical Mechanics". Harper \& Row. New York, 1976.

98. M. J. Frisch, A. Frisch and J. B. Foresman. "Manual de usuario del Programa Gaussian 94". Gaussian, Inc., Pittsburgh, PA, 1995.

99. N. M. Donahue, J. G. Anderson, and K. L. Dermejian. J. Phys. Chem. 102, 3121 , (1998).

100. D. L. Baulch, M. Bowers, D. G. Malcolm, R. T. Tuckerman. J. Phys. Chem. Ref. Data 15, 465, (1986).

101. N. Cohen and K. R. Westberg. J. Phys. Chem. Ref. Data 12, 531, (1983).

102. J. A. Jafri and D. H. Phillips. J. Am. Chem. Soc. 112, 2586, (1990).

103. D. Wu, and K. D. Bayes. Int. J. Chem. Kinet. 18, 547, (1986).

104. R. Atkinson, D. L. Baulch, R. A. Cox, R. F. Hampson Jr. J. Kerr, M. J. Rossi and J, J, Troe. J. Phys. Chem. Ref. Data, 26, (1987).

105. T, M. Lenhardt, C. E. McDade and K. D. Bayes. J. Chem. Phys. 72, 304, (1980).

106. R. A. Kenley, J. A Davenport, and D. G. Hendry. J. Phys. Chem. 85, 2740, (1981).

107. V. H. Uc, I. Garcia-Cruz, A. Hernández-Laguna and A. Vivier-Bunge. J. Phys. Chem. (1999), (Sometido).

108. I. Diaz-Acosta, J. R. Alvarez-Idaboy and A. Vivier-Bunge. Int. J. Chem. Kinet. 31 , 29, (1999).

109. J. R. Alvarez-Idaboy, I. Diaz-Acosta and A. Vivier-Bunge. J. Comp. Chem. 19, 811, (1999).

110. A. J. Kresge. Acc. Chem. Res. 8, 354, (1975).

111. S. S. Sahik, H. B. Schlegel and. S. Wolfe. "Theoretical Aspect of Physical Organic Chemistry. The $\mathrm{S}_{\mathrm{N}}^{2}$ Mechanism". John Wiley \& Sons, Inc. USA, 1992.

112. a) R. A. Marcus and N. Sutin. J. Chem. Phys. 24, 966, (1956), b) R. A. Marcus and N. Sutin. J. Chem. Phys. 24, 979, (1956), and c) R. A. Marcus and N. Sutin. Inorg. Chem. 5, 119, (1986).

113. R. Atkinson W. P. L. Carter and A. M. Winer. J. Phys. Chem. 87, 1605, (1983). 
114. P. B. Shepson, E. O. Edney and. E. W. Corse. J. Phys. Chem. 85, 296, (1977).

115. A. D. Becke. J. Chem. Phys. 98, 5648, (1993).

116. B. H. Besler, M. D. Sevilla, and P. McNeille. J. Phys. Chem. 90, 6446, (1986).

117. R. A. Baird, and W. A. Goddard III. J. Am. Chem. Soc. 104, 2719, (1982).

118. G. E. Quelch, M. M. Gallo, and H. F. Schaefer III. J. Am. Chem. Soc. 114, 8239, (1992).

119. J. A. Howard. Can. J. Chem. 57, 253, (1979).

120. V. Uc, I. Garcia-Cruz, and A. Vivier-Bunge. Progress in Theoretical Chemistry and Physics, (1999), (En prensa).

121. J. Yu, H. E. Jefries and K. G. Sexton. Atmos. Environ. 31, 2261, (1997).

122. J. Yu, H. E. Jefries and K. G. Sexton. Atmos. Environ. 31, 2281, (1997).

123. J. Yu. Doctoral Thesis. University of North Carolina at Chapel Hill. (1996). 